\author{
Universidade de São Paulo \\ Instituto de Física
}

\title{
Mistura de quatro ondas como fonte de estados emaranhados do campo
}

\author{
Bárbara Abigail Ferreira Ribeiro
}

Orientador: Prof. Dr.

Tese doutorado apresentada ao Instituto de Física para a obtenção do título de Doutor em Ciências

Banca Examinadora:

Prof. Dr. Marcelo Martinelli (IFUSP)

Prof. Dr. Mario José de Oliveira (IFUSP)

Prof. Dr. Ricardo E. Samad (IPEN)

Prof. Dr. Celso Vilas-Boas (UFSC))

Prof. Dr. Pablo L. Saldanha (UFMG) 


\section{FICHA CATALOGRÁFICA}

\section{Preparada pelo Serviço de Biblioteca e Informação}

do Instituto de Física da Universidade de São Paulo

Ribeiro, Bárbara Abigail Ferreira

Mistura de quatro ondas como fonte de estados emaranhados do campo. São Paulo, 2019.

Tese (Doutorado) - Universidade de São Paulo. Instituto de Física. Depto. Física Experimental.

Orientador: Prof. Dr. Marcelo Martinelli

Área de Concentração: Óptica Quântica, Informação Quântica.

Unitermos: 1. Óptica quântica; 2. Informação quântica; 3. Mecânica quântica. 


\title{
University of São Paulo \\ Physics Institute
}

\section{Four Wave Mixing as a source of entangles states of light}

\author{
Bárbara Abigail Ferreira Ribeiro
}

Supervisor: $\quad$ Prof. $\quad$ Dr. $\quad$ Marcelo Martinelli

Thesis submitted to the Physics Institute of the University of São Paulo in partial fulfillment of the requirements for the degree of Doctor of Science.

Examining Committee:

Prof. Dr. Marcelo Martinelli (IFUSP)

Prof. Dr. Dr. Mario José de Oliveira (IFUSP)

Prof. Dr. Dr. Ricardo E. Samad (IPEN)

Prof. Dr. Dr. Celso Vilas-Boas (UFSC)

Prof. Dr. Dr. Pablo L. Saldanha (UFMG) 

"Não é sobre chegar ao topo do mundo e saber que venceu. É sobre escalar e sentir que o caminho te fortaleceu." 


\section{Resumo}

A mistura de quatro ondas (4WM) é um processo não linear que permite a amplificação paramétrica de campos óticos. A partir de um campo de bombeio, podemos amplificar um campo de prova e gerar um campo conjugado neste processo com um elevado ganho. Recentemente, vários experimentos de 4WM foram realizados em células de rubídio revelando compressão de ruído e emaranhamento da luz $[1,2,3]$, levando a aplicações interessantes na área de informação quântica.

Estudamos a mistura de quatro ondas numa célula de vapor de rubídio dentro de uma cavidade ótica, servindo como um oscilador paramétrico ótico (OPO). Resolvemos o modelo de campo médio e estudamos as flutuações quânticas dos campos na operação acima do limiar de oscilação para os casos de ressonância dupla (prova e conjugado) e tripla.

Mostramos o emaranhamento de três campos (bombeio, prova e conjugado), além de compressão de ruído para o campo de bombeio e para os campos convertidos. Nosso modelo permite estudar o caso de uma cavidade aberta, explorando a região próxima ao limiar, diferente da situação experimental observada para sistemas com não-linearidades $\chi^{2}$, que geralmente possuem ganhos baixos e necessitam de cavidades fechadas.

palavras-chave: Ótica Quântica; Informação Quântica; Mecânica Quântica. 



\begin{abstract}
The four wave mixing $(4 \mathrm{WM})$ is a non-linear process that allows a parametric amplification of optical fields. From a pump field, we can amplify a test field and generate a conjugate field in this process with a high gain. Recently, several experiments of $4 \mathrm{WM}$ were carried out on rubidium cells revealing noise squeezing and entanglement [1, 2, 3], leading to some interesting aplications in the area of quantum information.

We have studied the four wave mixing in a rubidium vapor cell inside an optical cavity, acting as an optical parametric oscillator (OPO). We solved the mean field model and studied the quantum fluctuations of the fields in the operation above the oscillation threshold for the cases of double resonance (pump and conjugate) and triple (pump, probe and conjugate).

We show the entanglement of three fields (pump, probe and conjugate), as well as squeezing for the pump field and the converted fields. The model allows to study the case of an open cavity, exploring the region around the threshold, different from the experimental situation observed for non-linear systems $\chi^{2}$, which in general producess low gains and needs closed cavities.

palavras-chave: Quantum Optics; Quantum Information; Quantum Mechanics.
\end{abstract}





\section{Agradecimentos}

Nesses cinco anos de doutorado tive a oportunidade de crescer não só como cientista mas também como ser humano. E esse crescimento só foi possível graças as pessoa com quem dividi a minha vida. Não vou conseguir fazer todos o agradecimentos que gostaria, mas farei alguns agradecimentos especiais:

Iniciarei os agradecimentos pela minha família, cujos membros sempre me apoiaram nas decisões de vida que tomei e ofereceram conforto nos momentos difíceis. Agradeço aos meus pais João e Heloisa e a minha avó Carmelita, essas três pessoas são a base de tudo que sou hoje. Também gradeço aos meus irmãos Beatriz, Gabriel e Ariana por sua presença em minha vida e por tudo que compartilhamos.

Agradeço aos professores Marcelo Martinelli e ao professor Paulo Nussenzveig por me receberem no grupo. Agradeço especialmente ao professor Marcelo por sua orientação.

Agradeço a aos amigos e colegas Rayssa e Breno que participaram do desenvolvimento do trabalho teórico. Rayssa, obrigada por além de ser uma ótima parceira de trabalho ser também uma ótima amiga. Breno, você foi uma espécie de irmão mais velho nessa jornada, muito obrigada por todas as conversas que tivemos.

Agradeço também aos funcionários do IF por todo o auxilio ao longo do anos. Agradeço Eduardo (eletrônica) e ao Marcos (mecânica) por todos os equipamentos que vocês fizeram ou consertaram ao longo dos anos. Sem o trabalho de vocês, o meu trabalho seria impossível. Muito obrigada Edi, Luiz e Bianca pelo auxilio com a burocracia, vocês tornaram minha vida um pouco mais fácil.

Agradeço aos professores da banca examinadora, Mario José de Oliveira, Ricardo E. Samad, Celso Vilas Boas e Pablo L. Saldanha pela contribuição a versão final da tese.

Agradeço a equipe experimental, Hans, Harold e Álvaro por sua contribuição ao trabalho experimental.

Muito obrigada aos amigos de laboratório que fizeram os meus dias mais felizes e dividiram seu conhecimento comigo. Obrigada Renato, Luiz, Lucas, Igor, Túlio, Roger, klara, Renné, Pablo e Raul.

Agradeço também aos amigos que foram parte dessa jornada fora do laboratório. Obrigada especialmente ao Matheus e Natália. Também quero agradecer ao meu namorado Arthur por todo o seu carinho.

Agradeço ao CNPq pelo fomento. 



\section{Sumário}

1 Introdução $\quad 3$

2 Conceitos básicos 5

2.1 Campo eletromagnético quantizado . . . . . . . . . . . . . 5

2.2 Campo elétrico em termos da portadora e bandas laterais . . . . . . . . . . . 6

2.3 Quadraturas do campo elétrico . . . . . . . . . . . . . . . 9

2.4 Ruído quântico da luz . . . . . . . . . . . . . . . . . . . 9

2.5 Tomografia de estados quânticos . . . . . . . . . . . . . . . 12

2.6 Fotocorrente e sua relação com a flutuação de amplitude do campo elétrico . . . 13

2.7 Fotocorrente e quadraturas do campo . . . . . . . . . . . . . . . . . 14

2.8 Fotocorrente e demodulação . . . . . . . . . . . . . . . . . 16

2.9 Espectro de ruído . . . . . . . . . . . . . . . . . . . . 17

2.10 Detecção homodina . . . . . . . . . . . . . . . . . . 18

2.11 Cavidades Óticas . . . . . . . . . . . . . . . . . . . . . 21

2.11.1 Cavidade Fabry-Perot . . . . . . . . . . . . . . . 21

2.12 Detecção com cavidade de análise . . . . . . . . . . . . . . . 24

2.13 Emaranhamento . . . . . . . . . . . . . . . . . . 28

2.13 .1 Critério de Duan . . . . . . . . . . . . . . . . 30

2.13.2 Critério de positividade sob transposição parcial (PPT) . . . . . . . . 30

2.14 Mistura de quatro ondas . . . . . . . . . . . . . . . . . . 31

3 Estudo teórico do OPO com meio de ganho não linear de terceira ordem 35

3.1 Oscilador Paramétrico Ótico . . . . . . . . . . . . . . . 35

3.2 Ganho dos campos de prova e conjugado . . . . . . . . . . . . . 38

3.3 Campo médio do OPO duplamente ressonante . . . . . . . . . . . . . . . . . . . . . . . . . . . . . . .

3.4 Campo médio do OPO triplamente ressonante . . . . . . . . . . . . . 45

3.5 Ganho do 4WM em termos das bandas laterais . . . . . . . . . . . . . . 49

3.6 Volta dos feixes na cavidade . . . . . . . . . . . . . . 54

3.6.1 Matriz de covariância . . . . . . . . . . . . . . 56

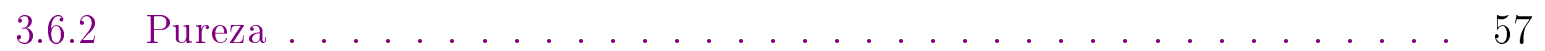


3.7 OPO duplamente ressonante na base simétrica e antissimétrica . . . . . . . 57

3.8 OPO duplamente ressonante na base soma e diferença . . . . . . . . . . . 61

3.9 Teste de positividade sob transposição parcial (DROPO) . . . . . . . . . 65

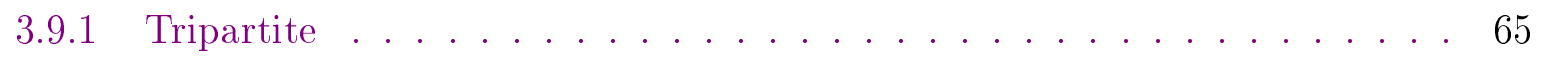

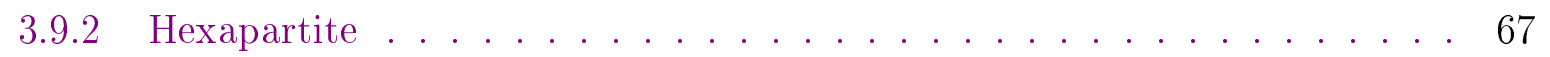

3.10 OPO triplamente ressonante na base simétrica e antissimétrica . . . . . . . 69

3.11 OPO triplamente ressonante na base soma e diferença . . . . . . . . . 73

3.12 Teste de positividade sob transposição parcial (TROPO) . . . . . . . . . . 76

3.12 .1 Tripartite . . . . . . . . . . . . . . . . . 76

3.12 .2 Hexapartite . . . . . . . . . . . . . . . . . 77

4 Experimento $\quad 80$

4.1 Implementação do experimento . . . . . . . . . . . . . . . . 80

4.2 Características do laser Ti:safira . . . . . . . . . . . . . . . 82

4.3 Características da célula de rubídio . . . . . . . . . . . . 83

4.4 Características do $\mathrm{OPO} \ldots \ldots \ldots \ldots . \ldots \ldots$

4.4.1 Campos gerados para célula de rubídio à $86^{\circ} \mathrm{C} \ldots \ldots \ldots$

4.4.2 Campos gerados para célula de rubídio à $60^{\circ} \mathrm{C} \ldots \ldots . \ldots 8$

4.5 Caracterização da cavidade de separação . . . . . . . . . . . . . . . 89

4.6 Caracterização do $1^{\circ}$ pico à célula de rubídio à $\mathrm{T}=86{ }^{\circ} \mathrm{C} \ldots \ldots \ldots$. . . . . . 90

4.7 Caracterização do $2^{\circ}$ pico $\mathrm{T}=60^{\circ} \mathrm{C} \ldots \ldots \ldots \ldots$. . . . . . . . . . . 92

4.8 Caracterização dos detectores disponíveis para as medidas do campo do $2^{\circ}$ pico

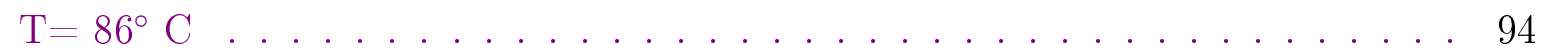

5 Conclusões e perspectivas $\quad 96$

$\begin{array}{ll}\text { A Dados ATFilms } & 98\end{array}$

$\begin{array}{ll}\text { B Geração da dessintonia } & 100\end{array}$

$\begin{array}{lll}\text { C Absorção saturada } & 102\end{array}$

$\begin{array}{ll}\text { D Acoplamento do Ti:safira a fibra ótica } & 105\end{array}$ 


\section{Capítulo 1}

\section{Introdução}

A área de ótica quântica é de grande interesse para a ciência de base por testar princípios de mecânica quântica tais como compressão de ruído [4] e emaranhamento [5], fenômenos intrinsecamente quânticos. Além disso, a ótica quântica tem potencial para ser uma interface para protocolos de informação quântica como criptografia quântica [6, 7], teleportação [8, 9, 10] e memória quântica [11].

Entre os mecanismos que podemos utilizar para produzir estados emaranhados está o oscilador paramétrico ótico (OPO) [12]. Esse mecanismo consiste de uma fonte de luz que incide em uma cavidade ótica com um meio de ganho não linear em seu interior. Sua configuração mais conhecida é o OPO com meio de ganho não linear $\chi^{2}$ que tem sido amplamente estudada tanto acima quanto abaixo do limiar [13, 14]. O estudo das propriedades dos OPOs $\chi^{2}$ é uma das principais linhas de pesquisa do grupo Laboratório de Manipulação Coerente de Átomos e Luz (LMCAL) do instituto de física da Universidade de São Paulo. Sendo o primeiro OPO $\chi^{2}$ de São Paulo montado pelo grupo e descrito na tese do professor Dr. Marcelo Martinelli [15]. E, nos diversos trabalhos posteriores seguiram os estudos sobre a caracterização das propriedades quânticas do OPO, tais como nos trabalhos [16], [17], culminando na primeira medida de emaranhamento tripartite entre os três feixes gerados [18]. Todos os estudos realizado tomaram como base o fato de que os estados estudados se comportavam como estados gaussianos [19].

A mistura de quatro ondas (four wave mixing 4WM) é um fenômeno da ótica não linear que ocorre em meios de ganho $\chi^{3}$. A configuração de 4WM discutida nessa tese consiste da aniquilação de dois fótons do bombeio e criação de um fóton no modo de prova e um fóton no modo conjugado, tal configuração é consistente com experimentos recentes de 4WM realizados em células vapor de rubídio. Tais experimentos geram campos com altos ganhos que apresentam compressão de ruído e emaranhamento [20, 21, 22]. Esses experimentos motivaram o grupo LMCAL utilizar células de vapor de rubídio como meio de ganho de um OPO $\chi^{3}$, pois os altos ganhos do 4WM permitem a utilização de cavidades mais abertas que possibilitam uma transição suave pelo limiar de oscilação do OPO. É esperado que os campos produzidos nessa região de transição apresentem comportamento não gaussiano. 
O trabalho apresentado nessa tese se insere nesse contexto como o primeiro trabalho do grupo LMCAL a estudar as flutuações quânticas de um OPO $\chi^{3}$ com um meio de ganho atômico. Nosso principal resultado é um modelo teórico, com aproximação para estados gaussianos, que estuda as flutuações quânticas do OPO $\chi^{3}$ nas proximidades do limiar. Esse trabalho é parte de um projeto maior que pretende utilizar o OPO $\chi^{3}$ como uma fonte de estados não gaussianos permitindo investigar novos sistemas que vão além dos que são permitidos pelos estados gaussianos [23, 24].

Essa tese está organizada de forma didática em seis capítulos onde o primeiro se refere a essa introdução que estamos fazendo. O capítulo 2 é uma breve revisão bibliográfica que tem por objetivo introduzir os conceitos fundamentais ao entendimento desse trabalho para o leitor iniciante e apresentar referências onde podem ser encontradas explicações mais detalhadas dos temas apresentados. Esse mesmo capítulo também serve para apresentar a notação que será utilizada ao longo do texto a um leitor com mais experiência na área. Na sequência, veremos o capítulo 3 onde se encontram os principais resultados dessa tese. Nesse capítulo apresentamos um modelo teórico para os campos médios do OPO duplamente ressonante (prova e conjugado) e triplamente ressonante (bombeio, prova e conjugado). Em seguida fazemos um estudo das flutuações quânticas com aproximação para estado gaussiano dos campos de bombeio, prova e conjugado. No capítulo 4 apresentamos os primeiros resultados experimentais desse projeto que serviram principalmente para apontar problemas que devem ser solucionados nas etapas que se seguem do projeto. Finalmente, no capítulo 5, fazemos um resumo dos principais resultados encontrados, apontamos os problemas que ainda precisam ser resolvidos sugerindo possíveis soluções. 


\section{Capítulo 2}

\section{Conceitos básicos}

Neste capítulo introduziremos conceitos fundamentais em ótica quântica que servirão de base para esse trabalho. Partindo do campo eletromagnético quantizado faremos um breve estudo sobre suas quadraturas. Definiremos o ruído quântico padrão, estados coerentes e comprimidos. Descreveremos a tomografia de estados quânticos enfatizando o caso gaussiano. Discutiremos o processo de demodulação dos dados para possibilitar o tratamento dos mesmos no espaço das frequências. Por fim explicaremos como funcionam as técnicas experimentais de detecção homodina e rotação da elipse de ruído que são utilizadas na parte experimental desse trabalho.

\subsection{Campo eletromagnético quantizado}

Como podemos ver em [25], [26] e outros, o campo eletromagnético pode ser visto como uma soma de infinitos modos de osciladores harmônicos independentes e, em sua forma quantizada, o operador campo elétrico é dado por:

$$
\hat{E}(\mathbf{r}, t)=\hat{E}^{(+)}(\mathbf{r}, t)+\hat{E}^{(-)}(\mathbf{r}, t)
$$

sendo

$$
\hat{E}^{(+)}(\mathbf{r}, t)=\sum_{\mathbf{k}, j} \hat{\epsilon}_{j} \mathscr{E}_{k} \hat{a}_{\mathbf{k}, j} e^{-i \omega_{k} t+i \mathbf{k} \cdot \mathbf{r}}, \quad \hat{E}^{(-)}(\mathbf{r}, t)=\left[\hat{E}^{(+)}(\mathbf{r}, t)\right]^{\dagger}
$$

onde $\mathbf{k}$ é o vetor de onda tal que $\mathbf{k} \equiv\left(k_{x}, k_{y}, k_{z}\right)$ está relacionado a frequência angular $\omega_{k}$ pela relação de dispersão $\omega_{k}=\frac{|\mathbf{k}|}{c}$, e cada vetor de onda pode assumir duas direções de polarização independentes $\hat{\epsilon}_{j}$ em que $j=(1,2)$. A constante $\mathscr{E}_{k}$ representa o campo elétrico de um único fóton sendo dada por:

$$
\mathscr{E}_{k}=\left(\frac{\hbar \omega_{k}}{2 \epsilon_{0} V}\right)^{1 / 2}
$$

em que $\epsilon_{0}$ é a permissividade do vácuo e $\mathrm{V}$ é o volume em que foi feita a quantização do campo. Os operadores $\hat{a}_{\mathbf{k}, j}$ e $\hat{a}_{\mathbf{k}, j}^{\dagger}$ são os operadores de aniquilação e criação de fótons, respectivamente, 
e obedecem às seguintes relações de comutação:

$$
\begin{aligned}
& {\left[\hat{a}_{\mathbf{k} j}, \hat{a}_{\mathbf{k}^{\prime} j^{\prime}}^{\dagger}\right]=\delta_{\mathbf{k k}^{\prime}} \delta_{j j^{\prime}},} \\
& {\left[\hat{a}_{\mathbf{k} j}, \hat{a}_{\mathbf{k}^{\prime} j^{\prime}}\right]=\left[\hat{a}_{\mathbf{k} j}^{\dagger}, \hat{a}_{\mathbf{k}^{\prime} j^{\prime}}^{\dagger}\right]=0 .}
\end{aligned}
$$

Qualquer possível combinação entre $m=\{\mathbf{k}, j\}$ forma um modo campo elétrico. É possível mostrar que o Hamiltoniano do campo eletromagnético quantizado é dado por:

$$
\begin{aligned}
\hat{H} & =\hbar \sum_{m} \omega_{m}\left(\hat{a}_{m}^{\dagger} \hat{a}_{m}+\frac{1}{2}\right) \\
& =\hbar \sum_{m} \omega_{m}\left(\hat{n}_{m}+\frac{1}{2}\right),
\end{aligned}
$$

onde $\hat{n}_{m} \equiv \hat{a}_{m}^{\dagger} \hat{a}_{m}$ é o operador que conta o número de fótons no modo $m$. Os autovalores do operador $\hat{n}_{m}$ são estados com número de fótons bem definidos dados por

$$
\left|\left\{n_{m}\right\}\right\rangle \equiv\left|n_{1}\right\rangle \otimes\left|n_{2}\right\rangle \otimes \ldots \otimes\left|n_{m^{\prime}}\right\rangle \ldots \otimes \ldots
$$

e o conjunto de todos os possíveis estados $\left|\left\{n_{m}\right\}\right\rangle$ formam a base dos estados número ou de Fock. Como o Hamiltoniano do campo eletromagnético pode ser escrito em função do operador número, os estados de Fock também são autovetores do Hamiltoniano do campo eletromagnético, porém com autovalores $E_{m}=\sum_{m} \hbar \omega_{m}\left(n_{m}+\frac{1}{2}\right)$.

\subsection{Campo elétrico em termos da portadora e bandas la- terais}

Para um feixe de luz de frequência central $\omega_{0}$ e largura de linha estreita, o modo de frequência $\omega_{0}$ é a portadora e todos os outros modos de frequência $\omega \neq \omega_{0}$ compõem os modos das bandas laterais. É usual descrever a frequência de cada modo das bandas laterais por meio de sua separação em relação à portadora, dessa forma, fazemos a mudança de variáveis $\Omega=\omega-\omega_{0}$. O campo de cada modo, inclusive da portadora, pode ser descrito por um valor médio mais uma flutuação $\left\langle\hat{a}_{\Omega}\right\rangle+\delta \hat{a}_{\Omega}$. Considerando que apenas os modos referentes à portadora são populados com uma quantidade apreciável de fótons, $\left\langle\hat{a}_{0}\right\rangle$ é o único termo que contribui para o valor médio da amplitude do campo, para todos os outros modos do campo $\left\langle\hat{a}_{(\Omega \neq 0)}\right\rangle \simeq 0$. As flutuações referentes à portadora $\delta \hat{a}_{0}$ são desprezadas nos cálculos devido à dificuldade de se medir flutuações em frequência nula. Nos modos referentes às bandas laterais a amplitude média do campo é desprezível, e portanto, $\hat{a}_{(\Omega \neq 0)}=\delta \hat{a}_{(\Omega \neq 0)}$. Uma representação pictórica dessa descrição é mostrada na figura 2.1 . 


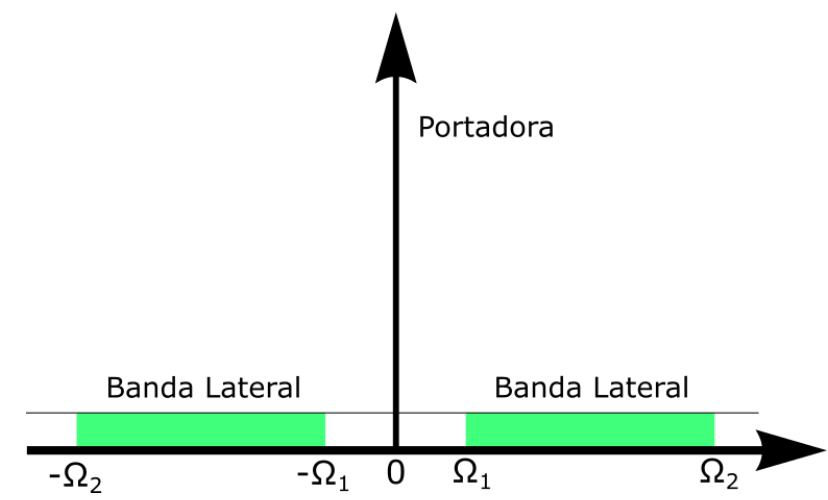

Figura 2.1: Representação do campo elétrico de um feixe de luz de frequência $\omega_{0}$ tal que $\omega_{0}=\frac{|k|}{c} \geq 0$ e largura de linha estreita. Para a portadora, $\Omega=0$, temos um campo intenso populado por uma grande quantidade de fótons. Para as bandas laterais, $-\Omega_{2}<\Omega<-\Omega_{1} \mathrm{e}$ $\Omega_{1}<\Omega<\Omega_{2}$ temos flutuações em torno de um campo médio nulo.

De forma geral, o operador $\hat{E}^{(+)}(t)$ é dado pela soma das contribuiçãoes dos diferentes modos do campo elétrico:

$$
\hat{E}^{(+)}(t)=\sum_{\omega} \mathscr{E}_{\omega} \hat{a}_{\omega} e^{-i \omega t},
$$

porém, considerando esse campo de largura de linha estreita, podemos separar as flutuações de cada modo de $\hat{E}^{(+)}(t)$ em uma flutuação rápida de frequência $\omega_{0}$ multiplicada por uma flutuação lenta de frequência $\left|\omega-\omega_{0}\right| \ll \omega_{0}$. Deixando a flutuação rápida em evidencia, temos:

$$
\hat{E}^{(+)}(t)=\mathscr{E}_{\omega_{0}}\left(\sum_{\omega} \hat{a}_{\omega} e^{-i\left(\omega-\omega_{0}\right) t}\right) e^{-i \omega_{0} t}
$$

em que utilizamos a aproximação $\mathscr{E}_{\omega} \approx \mathscr{E}_{\omega_{0}}$. Essa aproximação é válida pois o campo possui largura de linha estreita e portanto as flutuações da equação 2.3 ao redor da frequência $\omega_{0}$ são pequenas. Fazendo a mudança de variáveis $\Omega=\omega-\omega_{0}$ e observando que o operador $\hat{a}_{\omega}$ é equivalente à $\hat{a}_{\Omega}$, temos:

$$
\hat{E}^{(+)}(t)=\mathscr{E}_{\omega_{0}} \hat{a}(t) e^{-i \omega_{0} t}
$$

onde

$$
\hat{a}(t)=\sum_{\Omega} \hat{a}_{\Omega} e^{-i \Omega t}
$$

O somatório em $\Omega$ na equação 2.10 é discreto pois, devido à quantização do campo eletromagnético, as frequências $\Omega$ permitidas devem corresponder a frequências de ondas harmônicas contidas em um comprimento $L$ :

$$
\Omega(n)=\frac{2 \pi n}{c L}-\omega_{0}
$$

onde $n$ é um número $(0, \pm 1, \pm 2, \ldots)$. Sendo assim, a separação entre frequências nos modos $n$ 
e $n+1$ é dado por:

$$
\Delta \Omega=\Omega(n+1)-\Omega(n)=\frac{2 \pi}{c L} .
$$

Aumentando-se o comprimento da caixa, $L \rightarrow L+\Delta L$, diminuímos o incremento $\Delta \Omega$ de forma que quando $L \rightarrow \infty$, então $\Delta \Omega \rightarrow 0$. Nesse limite, deixamos de ter uma soma discreta de modos e passamos a ter uma soma contínua:

$$
\hat{a}(t)=\int_{-\omega_{0}}^{\infty} d \Omega \hat{a}_{\Omega} e^{-i \Omega t}
$$

Como o campo na frequência da banda lateral $\Omega \gg \omega_{0}$ não possuí um número de fótons apreciável, é possível aproximar o limite de integração $-\omega_{0}$ por $-\infty$ na equação 2.13. Dessa forma, reescrevemos $\hat{a}(t)$ como

$$
\hat{a}(t)=\int_{-\infty}^{\infty} d \Omega \hat{a}_{\Omega} e^{-i \Omega t} .
$$

Aqui vale inverter a equação 2.14 por meio da transformada de Fourier e decompor o operador $\hat{a}(t)$ no espaço das frequências

$$
\hat{a}_{\Omega} \equiv \int_{-\infty}^{\infty} d t \hat{a}(t) e^{i \Omega t}, \quad\left[\hat{a}_{\Omega}\right]^{\dagger}=\quad \hat{a}_{-\Omega}
$$

Tanto o estudo teórico quanto o estudo experimental das flutuações do campo elétrico se tornam mais fáceis quando separamos o valor médio do campo elétrico de suas flutuações. Matematicamente, para separar a frequência da portadora da frequência das bandas laterais na equação 2.14 utilizamos o símbolo $\int^{\prime}$ que representa a integral com limites entre $-\infty$ e $+\infty$, sem levar em conta a frequência da portadora. Dessa forma, $\hat{a}(t)$ pode ser reescrito como

$$
\hat{a}(t)=\alpha_{0}+\int^{\prime} d \Omega \hat{a}_{\Omega} e^{-i \Omega t}
$$

em que $\alpha_{0}$ representa o valor médio da amplitude da portadora, $\alpha_{0}=\left\langle\hat{a}_{0}\right\rangle$ e as flutuações ao redor desse valor médio são dadas pela contribuição das bandas laterais

$$
\delta \hat{a}(t) \equiv \int^{\prime} d \Omega \hat{a}_{\Omega} e^{-i \Omega t}
$$

cujo complexo conjugado é dado por

$$
\delta \hat{a}^{\dagger}(t) \equiv[\delta \hat{a}(t)]^{\dagger}=\int^{\prime} d \Omega \hat{a}_{\Omega}^{\dagger} e^{i \Omega t}=\int^{\prime} d \Omega \hat{a}_{-\Omega}^{\dagger} e^{-i \Omega t} .
$$




\subsection{Quadraturas do campo elétrico}

Os operadores de criação e aniquilação do campo elétrico não são observáveis. Assim, é interessante redefinir esse campo em termos de operadores hermitianos que realmente podem ser medidos. Dessa forma, apresentaremos nessa seção a descrição do campo elétrico em termos de quadraturas generalizadas. As informações que se seguem são baseadas nos textos [27], [28], os quais recomendo à leitura por apresentarem uma descrição mais completa e didática dos conceitos aqui apresentados.

Observando que cada modo do campo elétrico dado por 2.1 pode ser visto como uma onda plana, definimos as quadraturas generalizadas de cada modo do operador campo elétrico em um dado ponto do espaço como:

$$
\begin{aligned}
\hat{X}_{\theta}(t) & =\hat{a}(t) e^{-i \theta}+\hat{a}^{\dagger}(t) e^{i \theta}, \\
\hat{Y}_{\theta}(t) & =-i\left(\hat{a}(t) e^{-i \theta}-\hat{a}^{\dagger}(t) e^{i \theta}\right),
\end{aligned}
$$

em que $\theta$ é uma fase arbitrária equivalente à origem da contagem do tempo e as quadraturas $\hat{X}_{\theta}(t)$ e $\hat{Y}_{\theta}(t)$ representam duas amplitudes de oscilação do campo elétrico com uma diferença de fase de $\pi / 2$ entre si. Tais quadraturas obedecem à relação de comutação $\left[\hat{X}_{\theta}(t), \hat{Y}_{\theta}\left(t^{\prime}\right)\right]=$ $2 i \delta\left(t-t^{\prime}\right)$. Assim, podemos reescrever o campo elétrico quase monocromático em um dado ponto do espaço como:

$$
\hat{E}(t)=2 \mathscr{E} \hat{\epsilon}\left(\hat{X}_{\theta}(t) \cos \omega t+\hat{Y}_{\theta}(t) \operatorname{sen} \omega t\right) .
$$

Por questão de simplicidade de notação, omitiremos a dependência temporal dos operadores $\hat{X}_{\theta}$ e $\hat{Y}_{\theta}$ no restante do texto.

As quadraturas $\hat{X}_{\theta}(t)$ e $\hat{Y}_{\theta}(t)$ adquirem um significado especial quando a fase arbitrária $\theta$ coincide com a fase do campo elétrico $\varphi$. Quando $\theta=\varphi$ a quadratura $\hat{X}_{\varphi} \equiv \hat{p}$ representa a quadratura associada à amplitude do campo elétrico e a quadratura $\hat{X}_{\varphi} \equiv \hat{q}$ representa a quadratura associada à fase do mesmo.

\subsection{Ruído quântico da luz}

A ótica quântica investiga fenômenos envolvendo luz e sua interação com a matéria utilizando modelos físicos semi-clássicos ou quânticos. Em outras palavras, a ótica quântica aplica mecânica quântica ao estudo da luz [29]. Para explicar alguns fenômenos físicos tais como a catástrofe do ultravioleta e efeito fotoelétrico é necessário assumir a granulação da luz, ou seja, considerar que um feixe de luz é formado por quantas de energia, os fótons [30]. Uma forma de abordar as diferenças entre o campo clássico (onda eletromagnética) e o campo quântico (corrente de fótons) é por meio da estatística diferenciada que os fótons obedecem. Nessa seção estudamos o comportamento estatístico de dois tipos de estados da luz: estado coerente e es- 
tado comprimido. As informações aqui apresentadas se baseiam principalmente nos textos de [25] e [28].

Dado um observável $\hat{A}$ que atue em um estado $|\psi\rangle$, seu valor médio é dado por $\langle\psi|\hat{A}| \psi\rangle$ e as flutuações de suas medidas em relação ao valor médio são dadas pela variância, que é definida por $\Delta \hat{A}^{2} \equiv\left\langle\psi\left|\hat{A}^{2}\right| \psi\right\rangle-\langle\psi|\hat{A}| \psi\rangle^{2}$. Não é possível medir simultaneamente duas grandezas cujos operadores não comutam. O princípio da incerteza impõe um limite que se pode ter na previsão dos resultados das medidas dessas grandezas, dados dois observáveis $\hat{A}$ e $\hat{B}$ o produto de suas variâncias obedece a relação $\Delta \hat{A}^{2} \Delta \hat{B}^{2} \geq\left|\frac{1}{2 i}\langle[\hat{A}, \hat{B}]\rangle\right|^{2}$.

Utilizando a relação de comutação entre as quadraturas generalizadas do campo eletromagnético, concluímos que:

$$
\left(\Delta \hat{X}_{\theta}\right)^{2}\left(\Delta \hat{Y}_{\theta}\right)^{2} \geq 1
$$

Agora, vamos estudar as flutuações das quadraturas de alguns tipos especiais de campos eletromagnéticos. É possível mostrar que para o campo do vácuo as flutuações de quaisquer quadraturas do campo elétrico possuem incerteza mínima, ou seja:

$$
\left(\Delta \hat{X}_{\theta}\right)^{2}=\left(\Delta \hat{Y}_{\theta}\right)^{2}=1
$$

para qualquer valor de $\theta$. Essa flutuação característica é chamada de ruído quântico padrão.

Existe um outro tipo de estado populado com fótons que também é um estado de mínima incerteza, ou seja, as flutuações de suas quadraturas obedecem à equação 2.23. Essa classe de estados é conhecida como estados coerentes e podem ser definidos como auto-estados do operador de aniquilação:

$$
\hat{a}|\alpha\rangle=\alpha|\alpha\rangle
$$

em que $\alpha=|\alpha| e^{i \varphi}$ são os autovalores complexos do estado coerente, $|\alpha|$ representa a amplitude e $\varphi$ a fase do estado coerente.

Podemos reescrever o estado coerente $|\alpha\rangle$ na base de Fock:

$$
|\alpha\rangle=e^{\frac{-|\alpha|^{2}}{2}} \sum_{0}^{\infty} \frac{\alpha^{n}}{\sqrt{n !}}|n\rangle .
$$

Utilizando a expressão acima para calcular a probabilidade de se encontrar $n$ fótons no estado $\alpha$, encontramos que a estatística dos fótons do estado coerente obedece a distribuição de Poisson, dada por:

$$
P_{n}=e^{-\frac{\alpha^{2}}{2}} \frac{|\alpha|^{2}}{n !}
$$

Essa distribuição é característica de processos randômicos que só podem retornar números inteiros, como é o caso da flutuação na contagem de fótons pelo detector [31] e das flutuações da corrente elétrica [32]. 
Outra característica dos estados coerentes é que a variância do número de fótons desses estados é igual ao valor médio do mesmo:

$$
\Delta^{2} \hat{n}_{\alpha}=\left\langle\alpha\left|\hat{n}^{2}\right| \alpha\right\rangle-\langle\alpha|\hat{n}| \alpha\rangle=|\alpha|^{2}=\bar{n}
$$

Tais estados podem ser obtidos aplicando-se o operador deslocamento $\hat{D}(\alpha)=e^{\alpha \hat{a}^{\dagger}-\alpha^{*} \hat{a}}$ ao estado de vácuo $|0\rangle$ :

$$
|\alpha\rangle=\hat{D}(\alpha)|0\rangle
$$

Portanto, podemos ver os estados coerentes como uma forma deslocada do estado fundamental do campo eletromagnético, o estado do vácuo. Na figura 2.2 vemos uma representação gráfica da amplitude de um dado campo elétrico e de suas flutuações em termos das quadraturas generalizadas $\hat{X}$ e $\hat{Y}$, e das quadraturas associadas a amplitude $\hat{p}$ e a fase $\hat{q}$.

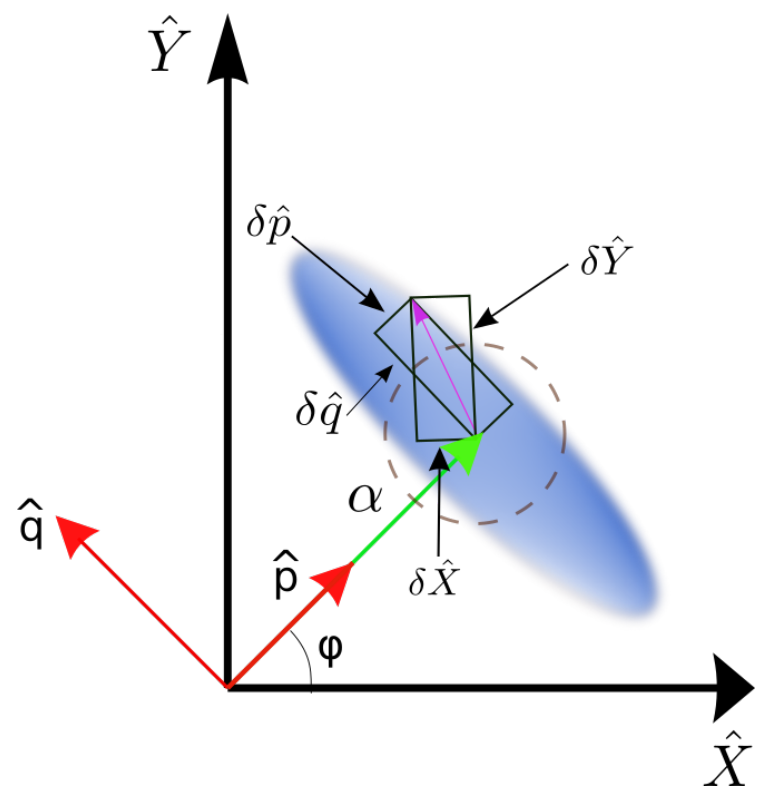

Figura 2.2: Os eixos $\hat{X}$ e $\hat{Y}$ representam duas quadraturas conjugadas do campo elétrico. Em verde vemos a amplitude do campo elétrico de módulo $\alpha$ e fase $\varphi$. O circulo pontilhado representa as flutuações de um estado coerente e a elipse representa a flutuação de um estado comprimido em amplitude. O vetor roxo representa a flutuação de uma quadratura generalizada do campo elétrico, essa flutuação pode ser decomposta nas flutuações das quadraturas $\hat{X}$ e $\hat{Y}$ que são representadas $\delta \hat{X}$ e $\delta \hat{Y}$ ou decomposta nas flutuações das quadraturas $\hat{p}$ e $\hat{q}$ representadas por $\delta \hat{p}$ e $\delta \hat{q}$.

Estados comprimidos são estados em que as flutuações de uma de suas quadraturas, por exemplo, $\Delta \hat{X}_{\theta}$, obedecem a relação:

$$
\left(\Delta \hat{X}_{\theta}\right)^{2}<1
$$

Vemos que as flutuações nessa quadratura sofrem uma compressão. Para não violar o princípio 
da incerteza a quadratura conjugada é "esticada":

$$
\left(\Delta \hat{Y}_{\theta}\right)^{2}>1
$$

Se, adicionalmente essas flutuações também obedecem a:

$$
\Delta \hat{X}_{\theta} \Delta \hat{Y}_{\theta}=1
$$

o estado é chamado estado comprimido ideal. Esses estados ideais podem ser obtidos por meio da aplicação de operadores de compressão $\hat{S}(\xi)=\exp \left(\frac{1}{2} \xi^{*} \hat{a}^{2}-\frac{1}{2} \xi \hat{a}^{\dagger 2}\right)$ em um estado coerente, que, por sua vez, foi obtido aplicando-se o operador de deslocamento no estado de vácuo:

$$
|\alpha, \xi\rangle=\hat{S}(\xi) \hat{D}(\alpha)|0\rangle
$$

\subsection{Tomografia de estados quânticos}

A tomografia de estados quânticos é o processo de recuperar a informação sobre um dado sistema. Essa informação está contida no vetor estado $|\psi\rangle$, no caso de estados puros ou na matriz densidade, $\hat{\rho}$, no caso de estados mistos. Assim, tomografar um estado quântico significa reconstruir sua matriz densidade possibilitando fazer a melhor previsão estatística possível sobre futuras interações do sistema.

Não é possível fazer uma medida direta da matriz densidade pois essa é definida em termos de operadores no espaço de Hilbert. Porém, a matriz densidade possui uma relação direta com a função de Wigner [33] definida por

$$
W(p, q)=\frac{1}{\pi} \int_{-\infty}^{\infty} d q^{\prime} e^{i q^{\prime} p}\left\langle q-q^{\prime}|\hat{\rho}| q+q^{\prime}\right\rangle
$$

Pela equação 2.33 vemos que a função de Wigner $\mathrm{W}(p, q)$ está associada univocamente à matriz densidade $\hat{\rho}$. As densidades de probabilidade das quadraturas do campo elétrico são as distribuições marginais da função de Wigner. Por exemplo, a distribuição marginal $\int_{-\infty}^{\infty} W(p, q) d p$ corresponde à probabilidade associada à quadratura fase $q$, e $\int_{-\infty}^{\infty} W(p, q) d q$ corresponde à probabilidade associada à quadratura amplitude $p$ [33].

Seja $\overrightarrow{\mathbf{X}}=\left(\begin{array}{lllll}\hat{p}_{\Omega} & \hat{q}_{\Omega} & \hat{p}_{\Omega^{\prime}} & \hat{q}_{\Omega^{\prime}} & \ldots\end{array}\right)$ o vetor que contém as quadraturas de amplitude $\hat{p}_{\Omega}$ e de fase $\hat{q}_{\Omega}$ dos modos de bombeio, prova e conjugado, as quadraturas $\hat{p}_{\Omega}$ e $\hat{q}_{\Omega}$ satisfazem a relação de comutação $\left[\hat{p}_{\Omega}, \hat{q}_{\Omega^{\prime}}\right]=2 i \delta\left(\Omega-\Omega^{\prime}\right)$ e se relacionam aos operadores $\hat{a}_{\Omega}$ e $\hat{a}_{\Omega}^{\dagger}$, que obedecem à relação de comutação $\left[\hat{a}_{\Omega}, \hat{a}_{\Omega^{\prime}}^{\dagger}\right]=\delta\left(\Omega-\Omega^{\prime}\right)$, por $\hat{p}_{\Omega}=\left(\hat{a}_{\Omega}+\hat{a}_{\Omega}^{\dagger}\right)$ e $\hat{q}_{\Omega}=-i\left(\hat{a}_{\Omega}-\hat{a}_{\Omega}^{\dagger}\right)$. Define-se os momentos de primeira ordem de $\overrightarrow{\mathbf{X}}$ como os elementos de

$$
\langle\overrightarrow{\mathbf{X}}\rangle=\operatorname{Tr}(\hat{\rho} \overrightarrow{\mathbf{X}})
$$


A análise de dados desse trabalho é feita na frequência das bandas laterais, de forma que $\langle\overrightarrow{\mathbf{X}}\rangle=\overrightarrow{0}$, pois, $\hat{p}_{\Omega}=\delta \hat{p}_{\Omega}$ e $\hat{q}_{\Omega}=\delta \hat{q}_{\Omega}$ para qualquer $\Omega \neq 0$. Os termos relevantes começam pelos momentos de segunda ordem. Esses termos são os elementos da matriz de covariância $\mathbf{V}$ definida como

$$
\mathbf{V}=\frac{1}{2}\left(\left\langle\overrightarrow{\mathbf{X}} \cdot \overrightarrow{\mathbf{X}}^{T}\right\rangle+\left\langle\overrightarrow{\mathbf{X}} \cdot \overrightarrow{\mathbf{X}}^{T}\right\rangle^{T}\right)
$$

em que elementos diagonais da matriz de covariância representam as variâncias das quadraturas do vetor $\overrightarrow{\mathbf{X}}$, como por exemplo, $\Delta \hat{p}_{\Omega} \equiv\left\langle\hat{p}_{\Omega} \hat{p}_{\Omega}\right\rangle$. Os elementos fora da diagonal representam as correlações entre quadraturas de diferentes modos ou entre quadraturas conjugadas de um mesmo modo, como por exemplo, $C\left(\hat{p}_{\Omega} \hat{p}_{\Omega^{\prime}}\right) \equiv\left\langle\hat{p}_{\Omega} \hat{p}_{\Omega^{\prime}}\right\rangle, C\left(\hat{p}_{\Omega} \hat{q}_{\Omega^{\prime}}\right) \equiv\left\langle\hat{p}_{\Omega} \hat{q}_{\Omega^{\prime}}\right\rangle$ e $C\left(\hat{p}_{\Omega} \hat{q}_{\Omega}\right) \equiv\left\langle\hat{p}_{\Omega} \hat{q}_{\Omega}\right\rangle$ [34].

Os estados da luz estudados nesse trabalho são estados cuja função de Wigner obedece a descrição gaussiana, ou seja, a função de Wigner depende apenas das flutuações dos momentos de primeira e segunda ordem. A função de Wigner para estados gaussianos de valor médio nulo é dada por [35]

$$
W(\overrightarrow{\mathbf{X}})=\frac{1}{\pi \sqrt{\operatorname{det} \mathbf{V}}} e^{-\frac{1}{2} \overrightarrow{\mathbf{x}}^{T} \mathbf{v}^{-1} \overrightarrow{\mathbf{x}}}
$$

A forma mais geral da matriz da covariância pode ser encontrada em [36]. Pela equação 2.36 podemos ver que para tomografar estados gaussianos necessitamos de no mínimo, um conjunto de medidas das quadraturas de amplitude $\hat{p}_{\Omega}, \hat{p}_{\Omega^{\prime}}, \ldots$ e um conjunto de medidas das quadraturas de fase $\hat{q}_{\Omega}, \hat{q}_{\Omega^{\prime}}, \ldots$. De modo mais geral, podemos dizer que no caso de variáveis contínuas precisamos medir as bases das variáveis canônicas que descrevem o espaço e no caso de variáveis discretas de um espaço de dimensão $N$ precisamos de um conjunto de medidas de, no mínimo, $N+1$ bases. Mais informações sobre tomografia de estados quânticos podem ser encontradas em [37].

\subsection{Fotocorrente e sua relação com a flutuação de ampli- tude do campo elétrico}

No laboratório, utilizamos fotodetectores que tem como princípio básico de funcionamento o efeito fotoelétrico. Ou seja, os fótons que atingem o detector são aniquilados enquanto fotoelétrons são criados. Dessa forma, os detectores geram uma corrente elétrica cuja intensidade é dada pelo número de fótons que atingem o detector no tempo. Podemos descrever a intensidade da fotocorrente em termos de um operador de fotocorrente [38]:

$$
\hat{I}(t)=\hat{a}^{\dagger}(t) \hat{a}(t)
$$


Os operadores $\hat{a}(t)$ e $\hat{a}^{\dagger}(t)$ podem ser descritos pelo seu valor médio, $\alpha=|\alpha| e^{-i \varphi} \equiv\langle\hat{a}(t)\rangle$, somado as flutuações do campo, $\delta \alpha$, em torno desse valor médio:

$$
\hat{a}(t)=\alpha+\delta \hat{a}(t), \quad \hat{a}^{\dagger}(t)=\alpha^{*}+\delta \hat{a}^{\dagger}(t)
$$

Expandiremos o operador de fotocorrente utilizando a expressão 2.38:

$$
\hat{I}(t)=|\alpha|^{2}+\alpha \delta \hat{a}^{\dagger}(t)+\alpha^{*} \delta \hat{a}(t)+\delta \hat{a}^{\dagger}(t) \delta \hat{a}(t)
$$

O primeiro termo de 2.39 representa o valor médio da fotocorrente enquanto os termos restantes representam a flutuação de $I(t)$. No entanto, o último termo dessa equação pode ser desprezado no caso de campos intensos, onde o valor de $|\alpha| \gg 1$. Dessa forma podemos reescrever a fotocorrente como:

$$
\hat{I(t)}=\langle\hat{I}(t)\rangle+\delta \hat{I}(t)
$$

onde $\langle\hat{I}(t)\rangle$ representa o valor médio do campo e $\delta \hat{I}(t)$ representa as flutuações do campo ao redor do valor médio:

$$
\langle\hat{I}(t)\rangle=|\alpha|^{2}, \quad \delta \hat{I}(t)=\alpha \delta \hat{a}^{\dagger}(t)+\alpha^{*} \delta \hat{a}(t)
$$

Dessa forma, podemos reescrever $\delta I(t)$ como:

$$
\begin{aligned}
\delta \hat{I}(t) & =|\alpha|\left[e^{-i \varphi} \delta \hat{a}(t)+e^{i \varphi} \delta \hat{a}^{\dagger}(t)\right] \\
& =|\alpha| \delta \hat{X}_{\varphi}(t) .
\end{aligned}
$$

Ou seja, a medida das flutuações da fotocorrente fornece um valor proporcional às flutuações da quadratura da amplitude do campo elétrico. Para conseguirmos informação sobre as demais quadraturas do campo elétrico precisamos utilizar alguma técnica de interferometria. Nesse trabalho utilizamos as técnicas de detecção homodina e rotação da elipse de ruído para caracterizar o nosso sistema. Essas técnicas de interferometria envolvem o estudo do campo elétrico no domínio da frequência.

\subsection{Fotocorrente e quadraturas do campo}

Como mencionado anteriormente, os fotodetectores irão aniquilar fótons e criar fotoelétrons dando origem a uma corrente elétrica proporcional ao número de fótons que os atingem. Essa fotocorrente pode ser separada em um valor médio $\langle\hat{I}(t)\rangle$ mais uma flutuação $\delta \hat{I}(t)$, ambos dados pela equação 2.41. O valor médio dessa expressão está associado ao módulo quadrado do valor médio do operador $\hat{a}(t)$, que, como visto na seção 2.6, é dado pela amplitude da portadora. 
Assim, temos

$$
\langle\hat{I}(t)\rangle=\alpha_{0}^{2}
$$

Lembrando que a frequência lentamente variável do campo da portadora corresponde a $\Omega=0$, podemos ver que $\langle\hat{I}(t)\rangle$ corresponde a uma corrente que flui apenas em um sentido. Esse tipo de corrente é denominada corrente contínua ou corrente DC (direct current).

As flutuações da fotocorrente, $\delta \hat{I}(t)$, estão associadas aos operadores $\delta \hat{a}(t)$ e $\delta \hat{a}^{\dagger}(t)$ dados pelas equações 2.17 e 2.18, respectivamente. Normalizando a fotocorrente por $|\alpha|=1$, por simplicidade, reescrevemos a equação 2.43 abrindo os termos em seu espectro de frequências

$$
\begin{aligned}
\delta \hat{I}(t) & =\left[\int^{\prime} d \Omega e^{-i \varphi} e^{-i \Omega t} \hat{a}_{\Omega}+\int^{\prime} d \Omega e^{i \varphi} e^{i \Omega t} \hat{a}_{\Omega}^{\dagger}\right] \\
& =\left[\int_{0}^{\infty} d \Omega\left(e^{-i \varphi} e^{-i \Omega t} \hat{a}_{\Omega}+e^{i \varphi} e^{i \Omega t} \hat{a}_{\Omega}^{\dagger}+e^{-i \varphi} e^{-i \Omega t} \hat{a}_{-\Omega}^{\dagger}+e^{i \varphi} e^{i \Omega t} \hat{a}_{-\Omega}^{\dagger}\right)\right] .
\end{aligned}
$$

Assim, vemos que a flutuação $\delta \hat{I}(t)$ é formada pelas contribuições dos operadores $\hat{a}_{\Omega}$ e $\hat{a}_{\Omega}^{\dagger}$ nos modos das bandas laterais, $\Omega \neq 0$. O campo elétrico correspondente a esses operadores produz uma corrente que oscila com frequência $\Omega$. Esse tipo de corrente é denominada corrente alternada ou corrente AC (alternating current).

Como estamos interessados em estudar as quadraturas do campo elétrico no espaço das frequências, definimos os operadores $\hat{p}_{ \pm \Omega}$ e $\hat{q}_{ \pm \Omega}$ das bandas superior e inferior:

$$
\begin{aligned}
& \hat{p}_{ \pm \Omega}=\hat{a}_{ \pm \Omega}+\hat{a}_{ \pm \Omega}^{\dagger}, \\
& \hat{q}_{ \pm \Omega}=-i\left(\hat{a}_{ \pm \Omega}-\hat{a}_{ \pm \Omega}^{\dagger}\right)
\end{aligned}
$$

tais operadores obedecem a relação de comutação $\left[\hat{p}_{\Omega}, \hat{q}_{\Omega^{\prime}}\right]=2 i \delta\left(\Omega-\Omega^{\prime}\right)$. Assim, a equação 2.45 pode ser reescrita em termos de 2.46 como:

$$
\begin{aligned}
\delta I(t)= & \cos (\varphi) \int_{0}^{\infty} d \Omega\left[\left(\hat{p}_{\Omega}+\hat{p}_{-\Omega}\right) \cos (\Omega t)+\left(\hat{q}_{\Omega}-\hat{q}_{-\Omega}\right) \operatorname{sen}(\Omega t)\right] \\
& +\operatorname{sen}(\varphi) \int_{0}^{\infty} d \Omega\left[\left(-\hat{p}_{\Omega}+\hat{p}_{-\Omega}\right) \operatorname{sen}(\Omega t)+\left(\hat{q}_{\Omega}+\hat{q}_{-\Omega}\right) \cos (\Omega t)\right] .
\end{aligned}
$$

Podemos ver que o sinal da fotocorrente fornecido pelos detectores fornece a informação da bandas superior e inferior atreladas entre si. Por esse motivo é interessante reescrever 2.47 em termos dos operadores $\hat{p}_{s / a}$ e $\hat{q}_{s / a}$ na base simétrica e antissimétrica. Tais operadores são definidos por:

$$
\begin{aligned}
\hat{p}_{s / a}(\Omega) & =\frac{1}{\sqrt{2}}\left(\hat{p}_{\Omega} \pm \hat{p}_{-\Omega}\right) \\
\hat{q}_{s / a}(\Omega) & =\frac{1}{\sqrt{2}}\left(\hat{q}_{\Omega} \pm \hat{q}_{-\Omega}\right) .
\end{aligned}
$$


Dessa forma, temos:

$$
\begin{aligned}
\delta \hat{I}(t)= & \cos (\varphi) \int_{0}^{\infty} d \Omega\left[\hat{p}_{s}(\Omega) \cos (\Omega t)+\hat{q}_{a}(\Omega) \operatorname{sen}(\Omega t)\right] \\
& +\operatorname{sen}(\varphi) \int_{0}^{\infty} d \Omega\left[-\hat{p}_{a}(\Omega) \operatorname{sen}(\Omega t)+\hat{q}_{s}(\Omega) \cos (\Omega t)\right]
\end{aligned}
$$

Da equação 2.49 podemos ver que a flutuação da fotocorrente carrega informação a respeito das quadraturas $\hat{p}_{s / a}(\Omega)$ e $\hat{q}_{s / a}(\Omega)$. Sendo assim, nossa próxima tarefa é encontrar uma maneira de extrair a informação sobre as variâncias e correlações entre as quadraturas $\hat{p}_{s / a}(\Omega)$ e $\hat{q}_{s / a}(\Omega)$ para podermos reconstruir a matriz de covariância.

\subsection{Fotocorrente e demodulação}

Como descrito anteriormente, é mais fácil realizar a análise de dados no domínio da frequência e para estudar os dados nesse domínio realizamos a demodulação do sinal temporal. Conforme descrito nos textos [28] e [34], tal procedimento consiste em misturar a fotocorrente $\hat{I}(t)$ a um sinal eletrônico periódico que oscile na frequência de análise desejada, como por exemplo: $I_{a n}(t)=e^{i \Omega_{a n} t}$. Em seguida fazemos a média temporal desse sinal. Matematicamente a demodulação equivale a:

$$
\begin{aligned}
\hat{I}_{\Omega_{a n}}=\int d t\left[\delta \hat{I}(t) I_{a n}(t)\right] & =\int^{\prime} d \Omega \int d t\left[e^{-i \varphi} e^{i\left(-\Omega+\Omega_{a n}\right) t} \hat{a}_{\Omega}+e^{i \varphi} e^{i\left(\Omega+\Omega_{a n}\right) t} \hat{a}_{\Omega}^{\dagger}\right] \\
& =\int^{\prime} d \Omega\left[e^{-i \varphi} \delta\left(-\Omega+\Omega_{a n}\right) \hat{a}_{\Omega}+e^{i \varphi} \delta\left(\Omega+\Omega_{a n}\right) \hat{a}_{\Omega}^{\dagger}\right] \\
& =e^{-i \varphi} \hat{a}_{\Omega_{a n}}+e^{i \varphi} \hat{a}_{-\Omega_{a n}}^{\dagger}=\hat{X}_{\varphi}\left(\Omega_{a n}\right)
\end{aligned}
$$

Como o sinal eletrônico $\hat{I}_{a n}(t)$ que foi misturado a fotocorrente não é hermitiano, o operador $\hat{I}_{\Omega_{a n}}$ também não é hermitiano. Podemos resolver esse problema dividindo o sinal em suas partes real e imaginária que tomam a forma:

$$
\hat{I}_{\Omega_{a n}}=\frac{1}{\sqrt{2}}\left(\hat{I}_{\cos \varphi}+i \hat{I}_{\operatorname{sen} \varphi}\right),
$$

onde:

$$
\begin{aligned}
\hat{I}_{\cos \varphi} & =\sqrt{2} \operatorname{Re}\left(\hat{I}_{\Omega_{a}}\right)=\frac{1}{\sqrt{2}}\left(\hat{I}_{\Omega_{a n}}+\hat{I}_{\Omega_{a n}}^{\dagger}\right) \\
& =\cos \varphi \hat{p}_{s}+\operatorname{sen} \varphi \hat{q}_{s} \\
\hat{I}_{s e n \varphi} & =\sqrt{2} \operatorname{Im}\left(\hat{I}_{\Omega_{a}}\right)=\frac{1}{\sqrt{2} i}\left(\hat{I}_{\Omega_{a n}}-\hat{I}_{\Omega_{a n}}^{\dagger}\right) \\
& =\cos \varphi \hat{q}_{a}-\operatorname{sen} \varphi \hat{p}_{a} .
\end{aligned}
$$


O operador $\hat{I}_{\cos \varphi}$ está associado a uma combinação linear dos operadores $\hat{p}_{s}$ e $\hat{q}_{s}$ enquanto $\hat{I}_{\text {sen }}$ está associado a uma combinação linear das quadraturas $\hat{p}_{a}$ e $\hat{q}_{a}$. Devido à geometria da representação dessas quadraturas nos espaços de fase das bandas simétricas e antissimétricas, definimos as quadraturas:

$$
\hat{X}_{s}^{\varphi} \equiv \hat{I}_{\cos \varphi}, \quad \hat{X}_{a}^{\varphi+\frac{\pi}{2}} \equiv \hat{I}_{s e n \varphi}
$$

Tais quadraturas estão em espaços de fase diferentes, o que significa que comutam entre si e portanto podem ser medidas simultaneamente. O processo experimental de medir as quadraturas $\hat{X}_{s}^{\varphi}$ e $\hat{X}_{a}^{\varphi+\frac{\pi}{2}}$ é relativamente simples, basta misturar o sinal $I(t)$ a dois sinais eletrônicos com uma fase de $\pi / 2$ entre si. A figura 2.3 ilustra esse procedimento.

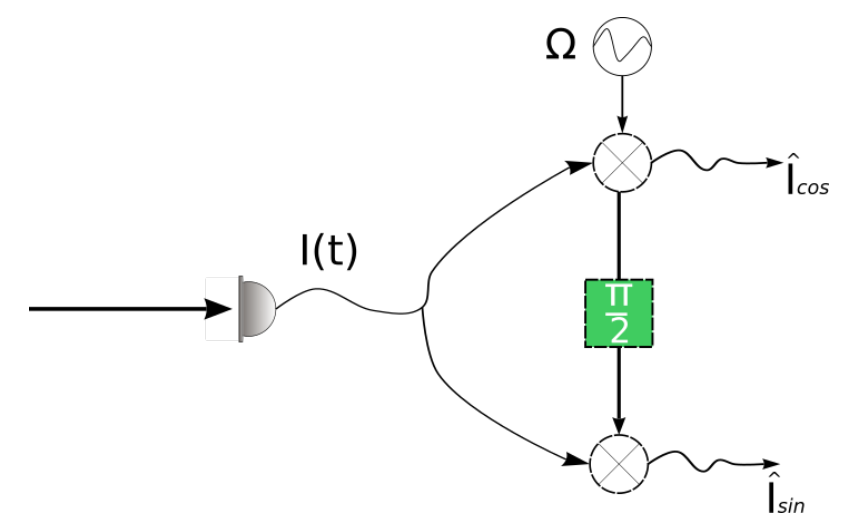

Figura 2.3: Esquema da medida das fotocorrentes em quadratura.

\subsection{Espectro de ruído}

Nesta seção fazemos uma breve discussão sobre o espectro de ruído baseada nos textos [28] e [34]. O espectro de ruído da fotocorrente, $S(\Omega)$, corresponde à correlação entre a fotocorrente medida em um dado instante $t$ e em um instante posterior $t^{\prime}=t+\tau$ para uma dada frequência de análise $\Omega$, ou seja:

$$
S(\Omega)=\int e^{i \Omega \tau}\langle\hat{I}(t) \hat{I}(t+\tau)\rangle d \tau
$$

Segundo o teorema de Wiener-Kintchine [39] para estados estacionários, ou seja, estados que a função de autocorrelação temporal, $C(t, t+\tau)=\langle\hat{I}(t) \hat{I}(t+\tau)\rangle$, depende apenas de $\tau$, o espectro de ruído é dado por:

$$
2 \pi S(\Omega) \delta\left(\Omega-\Omega^{\prime}\right)=\left\langle\hat{I}(\Omega) \hat{I}\left(-\Omega^{\prime}\right)\right\rangle
$$

que pode ser reescrito como:

$$
\begin{aligned}
& S(\Omega)=\left\langle\hat{I}(\Omega) \hat{I}\left(-\Omega^{\prime}\right)\right\rangle, \quad \Omega=\Omega^{\prime}, \\
& \left\langle\hat{I}(\Omega) \hat{I}\left(-\Omega^{\prime}\right)\right\rangle=0, \quad \Omega \neq \Omega^{\prime} .
\end{aligned}
$$


Podemos utilizar a equação 2.51 e a definição das quadraturas generalizadas da fotocorrente dada em 2.54 para reescrever o ruído estacionário de 2.57 como:

$$
S(\Omega)=\frac{1}{2}\left\langle\hat{I}_{\text {cos }}^{2}\right\rangle+\frac{1}{2}\left\langle\hat{I}_{\text {sen }}^{2}\right\rangle=\frac{1}{2} \Delta^{2} \hat{X}_{s}^{\varphi}+\frac{1}{2} \Delta^{2} \hat{X}_{a}^{\varphi+\frac{\pi}{2}}
$$

Utilizando a segunda parte de 2.57 para calcular $\left\langle\hat{I}_{\Omega} \hat{I}_{\Omega}\right\rangle$ mostramos que:

$$
\begin{aligned}
& \Delta^{2} \hat{X}_{s}^{\varphi}=\Delta^{2} \hat{X}_{a}^{\varphi+\frac{\pi}{2}} \\
& \left\langle\hat{X}_{s}^{\varphi} \hat{X}_{a}^{\varphi+\frac{\pi}{2}}\right\rangle=0 .
\end{aligned}
$$

Até o presente momento discutimos a relação entre a fotocorrente e o número de fótons detectados, a demodulação da fotocorrente e a relação entre a fotocorrente no espaço das frequências e o espectro de ruído. No entanto, a única grandeza que o detector é capaz de medir é a intensidade da luz. Sendo assim, as flutuações na intensidade medida estão relacionadas à quadratura amplitude do campo elétrico. Para termos informação sobre as demais quadraturas do campo elétrico necessitamos utilizar uma técnica interferométrica. As próximas seções tratarão das técnicas interferométricas experimentais utilizadas nesse trabalho.

\subsection{Detecção homodina}

Entre as técnicas que utilizaremos nesse experimento para estudar as flutuações das quadraturas do campo elétrico estão a detecção balanceada e a detecção homodina. Nesta seção é feita uma breve descrição dessas técnicas com base em [31], [40].

Vamos começar com a técnica de detecção balanceada, que serve para calibrar o ruído quântico padrão. Sabemos que a intensidade do feixe de um laser é proporcional ao número de fótons e que quando esses feixes atingem um detector a fotocorrente gerada passa a ser proporcional à intensidade do laser. Então, desconsiderando a constante de proporcionalidade entre a fotocorrente gerada e o número de fótons $\hat{I}_{\text {det }}=\hat{N}_{\text {det }}$.

A detecção balanceada é fruto da interferência entre o campo do vácuo e um campo coerente intenso. Para realizar a interferência entre dois campos, podemos utilizar um divisor de feixe (Beam splitter - BS). Os campos $\hat{a}$ e $\hat{b}$ representam os modos de entrada e os campos $\hat{c}$ e $\hat{d}$ representam os modos de saída em um BS. Para a detecção balanceada, o campo â é um campo coerente e intenso e o campo $\hat{b}$ é o campo do vácuo. O resultado dessa interferência são os campos de saída, $\hat{c}$ e $\hat{d}$, dados por:

$$
\hat{c}=\frac{1}{\sqrt{2}}(\langle\hat{a}\rangle+\delta \hat{a}+\delta \hat{b}),
$$




$$
\hat{d}=\frac{1}{\sqrt{2}}(\langle\hat{a}\rangle+\delta \hat{a}-\delta \hat{b}) .
$$

Os campos $\hat{c}$ e $\hat{d}$ emergem do BS e são detectados separadamente. A diferença entre as intensidades desses dois campos é proporcional à diferença entre seus operadores número e portanto proporcional à diferença entre as intensidades de fotocorrente $I_{c}$ e $I_{d}$, que é dada por:

$$
\begin{aligned}
\hat{I}_{-}^{\text {det B }} & =\hat{I}_{c}-\hat{I}_{d}=\hat{N}_{c}-\hat{N}_{d} \\
& \simeq \frac{1}{2}\left(|\alpha|^{2}+\alpha \delta \hat{a}^{\dagger}+\alpha \delta \hat{b}^{\dagger}+\alpha^{*} \delta \hat{a}+\alpha^{*} \delta \hat{b}\right)-\frac{1}{2}\left(|\alpha|^{2}+\alpha \delta \hat{a}^{\dagger}-\alpha \delta \hat{b}^{\dagger}+\alpha^{*} \delta \hat{a}-\alpha^{*} \delta \hat{b}\right) \\
& \simeq|\alpha|\left(e^{i \phi} \delta \hat{b}^{\dagger}+e^{-i \phi} \delta \hat{b}\right)=|\alpha| \delta \hat{X}_{p}^{b}
\end{aligned}
$$

desprezando as flutuações de segunda ordem. Vemos que a diferença entre as intensidades medidas é proporcional às flutuações das quadraturas do campo do vácuo, $\delta \hat{X}_{p}^{b}$, multiplicado pela amplitude do campo intenso $\alpha$. Conhecendo a intensidade do campo $\hat{a}$ podemos calcular o limite quântico padrão.

A soma das intensidades medidas nos dois detectores é dada por:

$$
\begin{aligned}
\hat{I}_{+}^{\text {det B }} & =\hat{I}_{c}+\hat{I}_{d}=\hat{N}_{c}+\hat{N}_{d} \\
& \simeq \frac{1}{2}\left(|\alpha|^{2}+\alpha \delta \hat{a}^{\dagger}+\alpha \delta \hat{b}^{\dagger}+\alpha^{*} \delta \hat{a}+\alpha^{*} \delta \hat{b}\right)+\frac{1}{2}\left(|\alpha|^{2}+\alpha \delta \hat{a}^{\dagger}-\alpha \delta \hat{b}^{\dagger}+\alpha^{*} \delta \hat{a}-\alpha^{*} \delta \hat{b}\right) \\
& \simeq|\alpha|^{2}+|\alpha|\left(e^{i \phi} \delta \hat{a}^{\dagger}+e^{-i \phi} \delta \hat{a}\right)=|\alpha|^{2}+|\alpha| \delta \hat{X}_{p}^{a},
\end{aligned}
$$

desprezando as flutuações de segunda ordem. Vemos que a soma das intensidades nos fornece um sinal proporcional à intensidade do campo $\hat{a}$. Com base nesse resultado, calculamos a variância de $\hat{I}_{+}^{\text {det } \mathrm{B} \text { : }}$

$$
\left(\Delta \hat{I}_{+}^{\operatorname{det} \mathrm{B}}\right)^{2}=|\alpha|^{2}\left\langle\left(\delta \hat{X}_{p}^{a}\right)^{2}\right\rangle=|\alpha|^{2}\left(\Delta \hat{X}_{p}^{a}\right)^{2}
$$

vemos que esta variância é proporcional à variância da quadratura de intensidade do campo. Esse método nos dá uma medida direta da quadratura de intensidade do campo $\hat{a}$. A figura 2.4 ilustra a detecção balanceada. Note que este mesmo resultado pode ser obtido com um único detetor. No entanto, a subtração fornece o sinal do ruido do vácuo como referência de um estado coerente, com a mesma cadeia de ganho ótico e eletrônico. Com isso podemos medir a flutuação de quadratura normalizada em unidades de flutuação do vácuo.

É possível observar que a detecção balanceada não oferece informações sobre as flutuações da quadratura de fase do campo â. Por esse motivo introduzimos o método da detecção homodina.

A Figura 2.5 ilustra um sistema de detecção homodina. Para realizar a detecção homodina necessitamos de um oscilador local, ou seja, campo coerente e intenso que sofra variação de fase, tal campo será representado por $E_{L O}$. Essa variação de fase pode ser produzida de diversas maneiras, uma forma muito simples de produzi-la é fazer com que o campo coerente incida em 


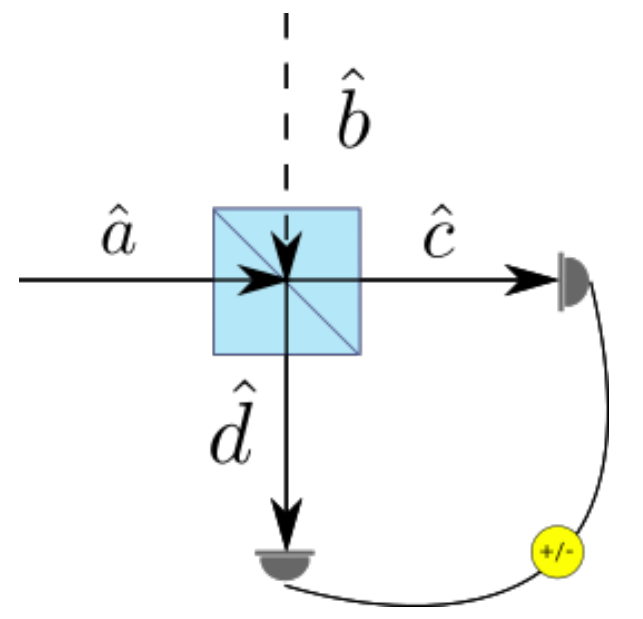

Figura 2.4: Detecção balanceada: O campo do qual deseja-se extrair as informações da quadratura intensidade é representado por $\hat{a}$ e incide em uma das faces do BS enquanto o campo do vácuo, que é representado por $\hat{b}$, incide pela outra face. O resultado da interferência entre $\hat{a}$ e $\hat{b}$ são os campos $\hat{c}$ e $\hat{d}$ que seguem rumo a detecção. Após esses campos serem detectados a soma e a subtração de suas intensidades são analisadas.

um espelho acoplado a um PZT ${ }^{1}$. Consideramos que $\left|E_{L O}\right|>>|\alpha|$, fazemos com que o campo que queremos estudar $\hat{a}$ interfira com o campo $\hat{b}$ (referente ao oscilador local $E_{L O}$ ) por meio de um BS. Detectamos os campos nas duas saída do BS e fazemos a subtração dos sinais:

$$
\begin{aligned}
\hat{I}_{-}^{\text {det } \mathrm{H}}= & \frac{1}{2}\left(\alpha+\delta \alpha^{\dagger}+\left|E_{L O}\right| e^{-i \phi}+\delta E_{L O}\right)\left(\alpha+\delta \alpha+\left|E_{L O}\right| e^{i \phi}+\delta E_{L O}\right)- \\
& \frac{1}{2}\left(\alpha+\delta \alpha^{\dagger}-\left|E_{L O}\right| e^{-i \phi}-\delta E_{L O}\right)\left(\alpha+\delta \alpha-\left|E_{L O}\right| e^{i \phi}-\delta E_{L O}\right) \\
\simeq & \left|E_{L O}\right|\left(\alpha e^{i \phi}+\alpha e^{-i \phi}\right)+\left|E_{L O}\right|\left(\delta \hat{a}^{\dagger} e^{i \phi}+\delta \hat{a} e^{-i \phi}\right)
\end{aligned}
$$

Para $\phi=0$ :

$$
\hat{I}_{-}^{\text {det H}} \simeq\left|E_{L O}\right|\left\langle\hat{X}_{p}^{a}\right\rangle+\left|E_{L O}\right| \delta \hat{X}_{p}^{a},
$$

e $\operatorname{para} \phi=\pi / 2$ :

$$
\hat{I}_{-}^{\text {det H}} \simeq\left|E_{L O}\right|\left\langle\hat{X}_{q}^{a}\right\rangle+\left|E_{L O}\right| \delta \hat{X}_{q}^{a} .
$$

Nesses cálculos, consideramos que $\left|E_{L O} \delta \hat{a}\right|>>\left|\hat{a} \delta E_{L O}\right|$. Varrendo-se a fase $\phi$ do oscilador local podemos ter acesso ao valor médio e às flutuações de qualquer quadratura do campo medido, em especial, fazendo a fase do oscilador local assumir os valores $\phi=0$ ou $\phi=\pi / 2$ podemos ter uma medida direta das quadraturas de intensidade, representada pelo subíndice $p$, ou de fase, representada pelo subíndice $q$, respectivamente.

\footnotetext{
${ }^{1} \mathrm{O}$ titanato zirconato de chumbo, PZT, um material cerâmico que possui efeito piezoelétrico e expande com a aplicação de tensão.
} 


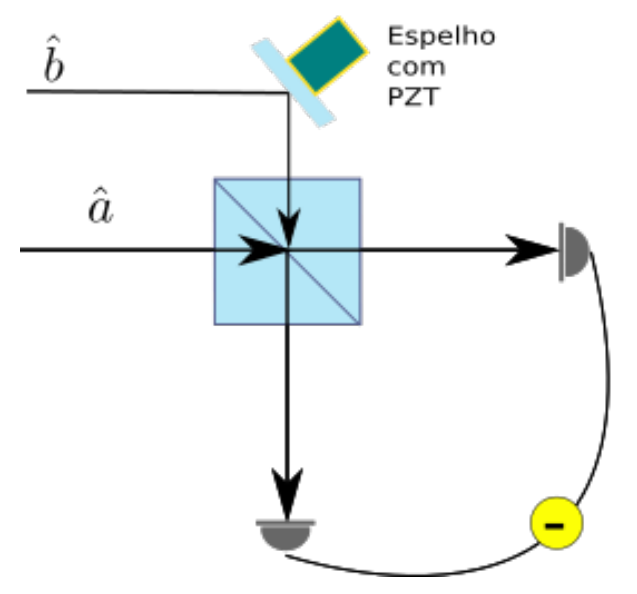

Figura 2.5: Detecção homodina: O campo do qual desejamos extrair as informações sobre suas quadraturas é representado por $\hat{a}$ e incide em uma das faces do BS enquanto o campo coerente e intenso $\hat{b}$ incide em um espelho acoplado a um PZT que varia sua fase, e, em seguida, incide na outra face do BS. O resultado da interferência entre $\hat{a}$ e $\hat{b}$ são os campos $\hat{c}$ e $\hat{d}$ que seguem rumo a deteç̧ão. Após serem detectados esses campos passam por uma análise de dados que envolve a subtração de suas intensidades.

\subsection{Cavidades Óticas}

Cavidades óticas são um conjunto de espelhos devidamente dispostos de forma que possam confinar ondas eletromagnéticas. Uma boa maneira de introduzir os principais parâmetros que devemos conhecer quando estudamos cavidades óticas, assim como a relação dentre os campos de entrada e saída da cavidade, é por meio do estudo de uma cavidade linear vazia, tal cavidade é denominada cavidade Fabry- Perot e devido a versatilidade de aplicações dessa cavidade existe uma vasta bibliografia sobre o assunto [41, 42]. Os parâmetros que serão apresentados para a cavidade Fabry-Perot são os mesmos parâmetros que utilizamos para cavidades com outras geometrias, como por exemplo cavidades triangulares, que nesse trabalho foram utilizadas para separar feixes de frequências distintas (cavidade de separação) e para a técnica de interferometria de rotação de elipse (cavidade de análise).

\subsubsection{Cavidade Fabry-Perot}

A Figura 2.6 ilustra uma cavidade Fabry-Perot que consiste de dois espelhos paralelos parcialmente refletores separados por uma distância L/2. Nessa figura vê-se dois espelhos de reflectâncias $R_{1}$ e $R_{2}$ e os campos resultantes da incidência do campo de entrada $E_{\text {in }}$ no espelho $R_{1}$. O campo $E_{R}$ é o campo resultante da soma da parcela refletida de $E_{i n}$ com a parcela transmitida de $E_{c^{\prime}}$ através do espelho $R_{1}$, e o campo $E_{T}$ é o campo resultante da transmissão 
de $E_{c}$. Os campos $E_{c}$ e $E_{c^{\prime}}$ são os campos resultantes das múltiplas reflexões da parcela de $E_{i n}$ que é transmitida para o interior da cavidade.

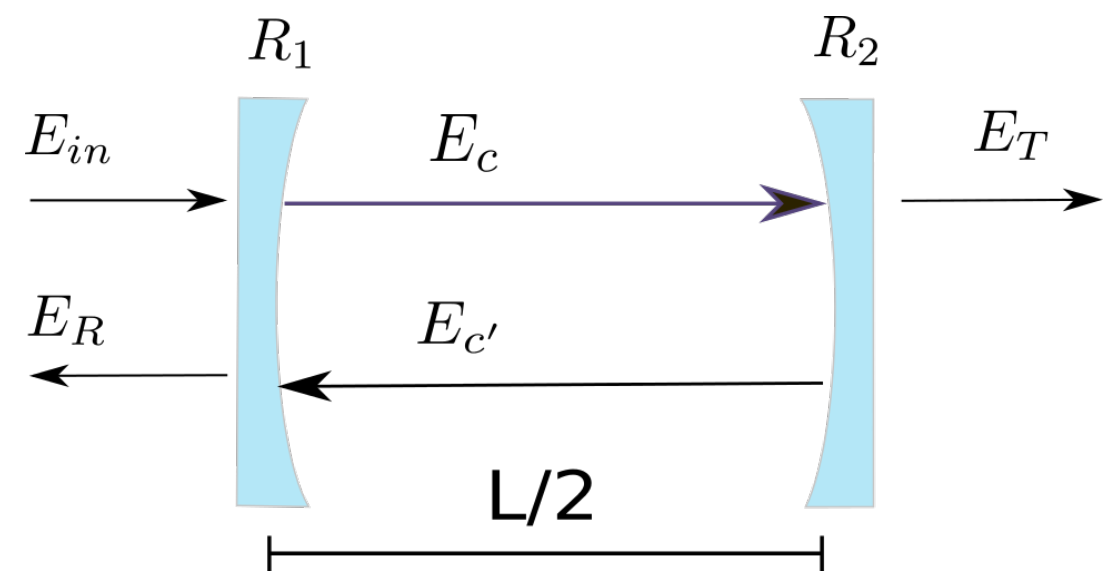

Figura 2.6: Cavidade Fabry-Perot: Dois espelhos parcialmente refletores paralelos com reflectâncias $R_{1}$ e $R_{2}$ separados por uma distância L/2. Vê-se o campo $E_{\text {in }}$ entrando na cavidade, os campos intra cavidade $E_{c}$ e $E_{c^{\prime}}$, o campo transmitido $E_{T}$ e o campo refletido $E_{R}$.

As multiplas reflexões nos espelhos de reflectância $R_{1}$ e $R_{2}$ geram um coeficiente de reflexão efetivo $R$ da cavidade. A reflectância $R$ é definida como a fração da potência de entrada da onda eletromagnética que é refletida pela cavidade e, de forma análoga, define-se a transmitância $T$ como a fração da potência onda eletromagnética incidente que é transmitida pela cavidade. Como a potência refletida somada à transmitida deve ser igual à potência incidente, concluí-se que $T+R=1$ na ausência de perdas espúrias. Quando se trabalha com as amplitudes do campo eletromagnético é mais comum utilizar os coeficientes de reflexão, $r$, e transmissão, $t$ da cavidade que podem ser definidos em termos da reflectância e da transmitância por $r=\sqrt{R}$ e $t=\sqrt{T}$. Sendo $k=\frac{2 \pi}{\lambda}$ o número de onda para um campo de comprimento de onda $\lambda$, as amplitudes dos campos de saída $E_{R}$ e $E_{T}$ se relacionam às do campo de entrada $E_{\text {in }}$ da cavidade por:

$$
\begin{aligned}
& E_{T}=t(L) E_{i n}, \quad \text { onde } t(L)=\frac{t_{1} t_{2} e^{-i k L / 2}}{1-r_{1} r_{2} e^{-i k L}}, \\
& E_{R}=r(L) E_{i n}, \quad \text { onde } r(L)=\frac{r_{1}-r_{2} e^{-i k L}}{1-r_{1} r_{2} e^{-i k L}}
\end{aligned}
$$

em que $T_{i}=1-R_{i}, r_{i}=\sqrt{R_{i}}$ e $t_{i}=\sqrt{T_{i}}$ correspondem a transmitância, coeficiente de reflexão e coeficiente de transmissão do espelho $i=1,2$.

Sabendo que a intensidade do campo refletido é dada por $I_{R}=\left|E_{R}\right|^{2}=R\left|E_{\text {in }}\right|^{2}$ e a intensidade do campo transmitido é dada por $I_{T}=\left|E_{T}\right|^{2}=T\left|E_{\text {in }}\right|^{2}$ utiliza-se as Equações 2.68 e 2.69 para chegar a:

$$
T=\frac{\sqrt{\left(R_{1}-1\right)^{2}\left(R_{2}-1\right)^{2}}}{-2 \sqrt{R_{1} R_{2}} \cos (k L)+R_{1} R_{2}+1} \quad \text { e } \quad R=\frac{-\sqrt{R_{1} R_{2}} \cos (k L)+R_{1}+R_{2}}{-2 \sqrt{R_{1} R_{2}} \cos (k L)+R_{1} R_{2}+1} .
$$


Após estabelecer como se comportam os campos refletidos e transmitidos pela cavidade iremos definir os principais parâmetros utilizados para caracterização da mesma. Entre esses parâmetros está a finesse, $F$, essa grandeza está associada às perdas da cavidade e pode ser interpretada como uma medida da nitidez da transmissão da cavidade. A finesse é calculada como:

$$
F=\pi\left[2 \operatorname{arcsen}\left(\frac{1-\sqrt{R_{1} R_{2}}}{2\left(R_{1} R_{2}\right)^{1 / 4}}\right)\right]^{-1} \approx \frac{\pi\left(R_{1} R_{2}\right)^{1 / 4}}{1-\sqrt{R_{1} R_{2}}} .
$$

Outro parâmetro importante é o intervalo espectral livre, $\Delta \omega_{c}$, que define a distância espectral entre duas ressonâncias consecutivas:

$$
\Delta \omega_{c}=\frac{c}{L}
$$

De 2.72 é fácil ver que o intervalo espectral livre também corresponde ao inverso do tempo de voo da frente de onda no interior da cavidade.

Por meio da razão entre o intervalo espectral livre e a finesse calcula-se a largura de banda, $\delta \omega_{c}$, que é definida como a largura a meia altura do pico de ressonância do espectro de frequência da cavidade:

$$
\delta \omega_{c}=\frac{\Delta \omega_{c}}{F} .
$$

Sendo $\omega_{0}$ a frequência da portadora e $\omega_{c}$ a frequência de ressonância da cavidade, a dessintonia normalizada pela largura de banda é dada por:

$$
\Delta=\frac{\omega_{0}-\omega_{c}}{\delta \omega_{c}}
$$

É interessante reescrever os coeficientes de transmissão e reflexão em termos da largura de banda e da dessintonia da cavidade, desse modo, seguem as relações:

$$
t(\Delta)=\frac{t_{1} t_{2} e^{\frac{i 2 \pi \Delta}{F}}}{1-r_{1} r_{2} e^{\frac{i 2 \pi \Delta}{F}}} \text { e } r(\Delta)=\frac{r_{1}-r_{2} e^{\frac{i 2 \pi \Delta}{F}}}{1-r_{1} r_{2} e^{\frac{i 2 \pi \Delta}{F}}} .
$$

Definimos a transmissão e a reflexão da cavidade como $R=|r(\Delta)|^{2}$ e $T=|t(\Delta)|^{2}$. Quando a dessintonia entre o campo e a cavidade é nula, $\Delta=0$, a reflexão atinge seu valor mínimo e consequentemente, a transmissão atinge seu valor máximo sendo dadas por:

$$
R_{\min }=\frac{\left(\sqrt{R_{1}}-\sqrt{R_{2}}\right)^{2}}{\left(1-\sqrt{R_{1} R_{2}}\right)^{2}} \text { e } T_{\max }=1-R_{\min } .
$$

Para altas finesses, podemos aproximar $F=2 \pi /\left(T_{1}+T_{2}\right)$ e expandir os parametros $r(\Delta)$ e $t(\Delta)$ em torno do valor da ressonância:

$$
r(\Delta)=-\sqrt{R_{\min }} \frac{1-2 i \Delta / \sqrt{R_{\min }}}{1+2 i \Delta} \text { e } t(\Delta)=\sqrt{T_{\max }} \frac{1}{1+2 i \Delta} .
$$


A figura 2.7 representa o modulo ao quadrado, $|r(\Delta)|^{2}$, e fase, $e^{i \theta_{R}}=r(\Delta) /|r(\Delta)|$, do coeficiente de reflexão da cavidade $r(\Delta)$ para $R_{1}=0.95$ e $R_{2}=0.997$.

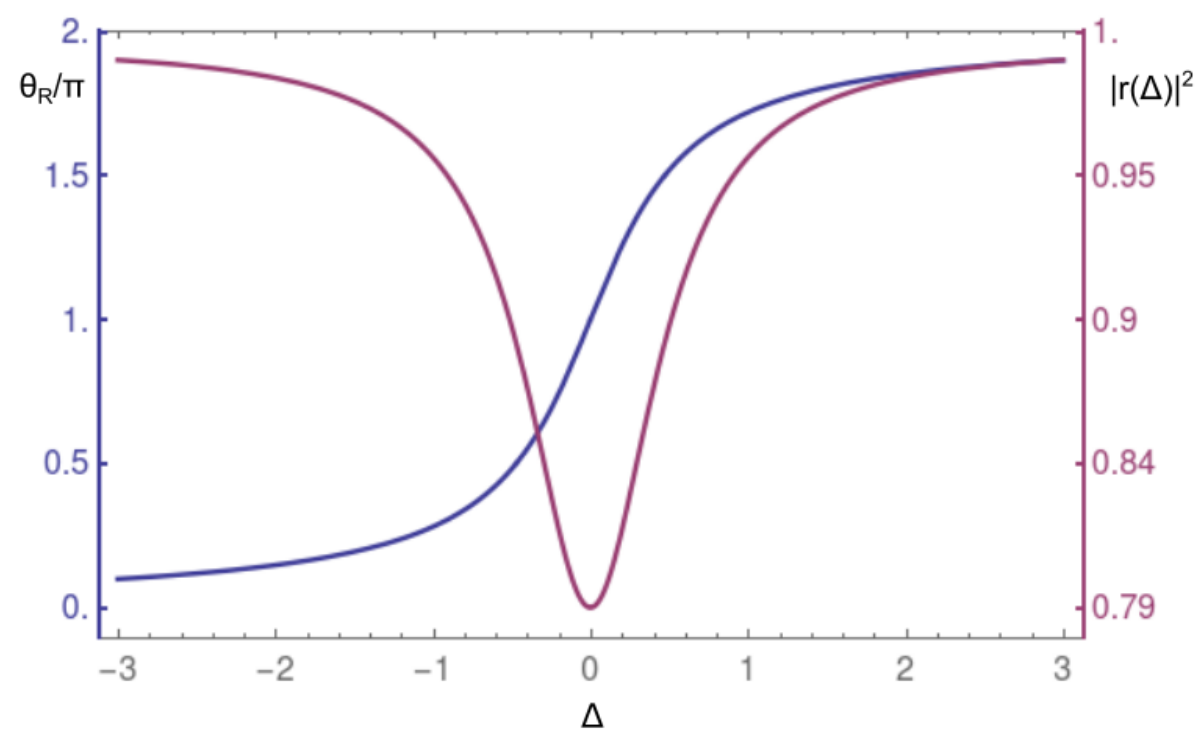

Figura 2.7: Modulo ao quadrado e fase do coeficiênte de reflexão da cavidade $r(\Delta)$ para $R_{1}=$ 0.95 e $R_{2}=0.997$.

\subsection{Detecção com cavidade de análise}

Essa seção se baseia principalmente no texto [27], mas também utilizamos informações dos textos [28] e [34] quando julgamos que estes expõem o assunto de forma mais didática. Na técnica de detecção com cavidade de análise a portadora do campo é utilizada como oscilador local. Por meio de uma cavidade ótica provocamos a rotação da elipse de ruído em relação ao campo da portadora. Assim, projetamos as flutuações de fase em flutuações de amplitude.

Lembrando que $\Omega=\omega-\omega_{0}$, onde $\omega_{0}$ é a frequência da portadora, o operador de aniquilação $\hat{a}_{\Omega R}$ referente ao campo de frequência $\Omega$ refletido pela cavidade vazia e seu complexo conjugado, $\hat{a}_{-\Omega R}^{\dagger}$, são modelados por:

$$
\begin{aligned}
\hat{a}_{\Omega R} & =r\left(\Delta_{\Omega}\right) \hat{a}_{\Omega i n}+t\left(\Delta_{\Omega}\right) \hat{b}_{\Omega}, \\
\hat{a}_{-\Omega R}^{\dagger} & =r^{*}\left(\Delta_{-\Omega}\right) \hat{a}_{-\Omega i n}^{\dagger}+t^{*}\left(\Delta_{-\Omega}\right) \hat{b}_{-\Omega}^{\dagger},
\end{aligned}
$$

onde $\hat{a}_{\Omega i n}$ é o operador de aniquilação do campo do frequência $\Omega$ na entrada da cavidade e $\hat{a}_{\Omega i n}^{\dagger}$ é o seu complexo conjugado, $\hat{b}_{\Omega}$ é o operador de aniquilação do vácuo para as frequências $\Omega$ e $\hat{b}_{\Omega}^{\dagger}$ é seu complexo conjugado, $\Delta_{ \pm \Omega}=\frac{\omega_{0}-\omega_{c} \pm \Omega}{\delta \omega_{c}}$ é a dessintonia normalizada pela largura de banda 
entre a frequência de ressonância da cavidade e as frequências $\omega_{0} \pm \Omega$, os parâmetros $r\left(\Delta_{ \pm \Omega}\right)$ e $t\left(\Delta_{ \pm \Omega}\right)$ são os coeficientes de reflexão e de transmissão da cavidade para as dessintonias $\Delta_{ \pm \Omega}$.

Definimos as quadraturas amplitude e fase do campo elétrico para uma dada frequência $\Omega$ como:

$$
\begin{aligned}
& \hat{p}_{\Omega}=e^{-i \varphi} \hat{a}_{\Omega}+e^{i \varphi} \hat{a}_{-\Omega}^{\dagger}, \\
& \hat{q}_{\Omega}=-i\left[e^{-i \varphi} \hat{a}_{\Omega}-e^{i \varphi} \hat{a}_{-\Omega}^{\dagger}\right] .
\end{aligned}
$$

Onde $\varphi$ é a fase do campo elétrico. Sendo assim, a quadratura amplitude em frequência $\Omega$ do campo elétrico refletido por uma cavidade é dada por:

$$
\hat{p}_{\Omega R}=e^{-i \theta_{R}(\Delta)} \hat{a}_{\Omega R}+e^{i \theta_{R}(\Delta)} \hat{a}_{-\Omega R}^{\dagger}
$$

em que a fase $e^{i \theta_{R}(\Delta)}=r(\Delta) /|r(\Delta)|$ corresponde à fase que a portadora do campo acumula após a reflexão pela cavidade. Note que o operador $\hat{p}_{\Omega R}$ não é hermitiano. Trata-se de uma combinação de banda lateral superior e inferior, retornando ao final um operador associado a uma fotocorrente. Os termos hermitianos irão corresponder, no final às quadraturas do sinal elétrico demodulado por um sinal de frequência $\Omega$.

Substituindo as equações 2.78 e 2.79 na expressão da quadratura amplitude refletida, 2.82, temos:

$$
\begin{aligned}
\hat{p}_{\Omega R}= & e^{-i \theta_{R}(\Delta)} r\left(\Delta_{\Omega}\right) \hat{a}_{\Omega i n}+e^{i \theta_{R}(\Delta)} r^{*}\left(\Delta_{-\Omega}\right) \hat{a}_{-\Omega i n}^{\dagger} \\
& +e^{-i \theta_{R}(\Delta)} t\left(\Delta_{\Omega}\right) \hat{b}_{\Omega}+e^{i \theta_{R}(\Delta)} t^{*}\left(\Delta_{-\Omega}\right) \hat{b}_{-\Omega}^{\dagger} .
\end{aligned}
$$

Por hora, vamos considerar as perdas espúrias iguais a zero, o que implica em $\left|r\left(\Delta_{ \pm \Omega}\right)\right|=1$ e $t\left(\Delta_{ \pm \Omega}\right)=0$. Para realizar a rotação de elipse precisamos garantir que a frequência de análise, $\Omega$, é muito maior que a largura de banda da cavidade, $\delta \omega_{c}$. Dessa forma, quando a cavidade estiver próxima à ressonância com a banda superior (inferior), irá atuar apenas na banda superior (inferior) provocando sua rotação:

$$
\begin{aligned}
& \hat{p}_{\Omega R}=e^{-i \theta_{R}\left(\Delta_{\Omega}\right)} \hat{a}_{\Omega i n}+\hat{a}_{-\Omega i n}^{\dagger}, \quad \text { (rotação da banda superior) } \\
& \hat{p}_{\Omega R}=\hat{a}_{\Omega i n}+e^{i \theta_{R}\left(\Delta_{-\Omega}\right)} \hat{a}_{-\Omega i n}^{\dagger} .
\end{aligned}
$$

Caso seja a frequência da portadora que está próxima à ressonância, a cavidade irá atuar principalmente sobre a portadora fazendo variar a fase $e^{i \theta_{R}(\Delta)}$ da equação 2.83. Devido à consideração das perdas espúrias iguais a zero, esse caso se reduz a:

$$
\hat{p}_{\Omega R}=e^{-i \theta_{R}(\Delta)} \hat{a}_{\Omega i n}+e^{i \theta_{R}(\Delta)} \hat{a}_{-\Omega i n}^{\dagger}
$$


A figura 2.8 ilustra a maneira como a cavidade de análise atua separadamente em cada banda lateral e na portadora enquanto é varrida. Na parte (a) a cavidade de análise está próxima à ressonância com a banda lateral inferior e atua girando a elipse de ruído mas sem modificar a fase da portadora, na parte (b) a cavidade está próxima à ressonância com a portadora de forma que a amplitude do campo é girada mantendo a elipse de ruído fixa. A parte (c) da figura é análoga a parte (a), com a cavidade atuando na banda lateral superior.

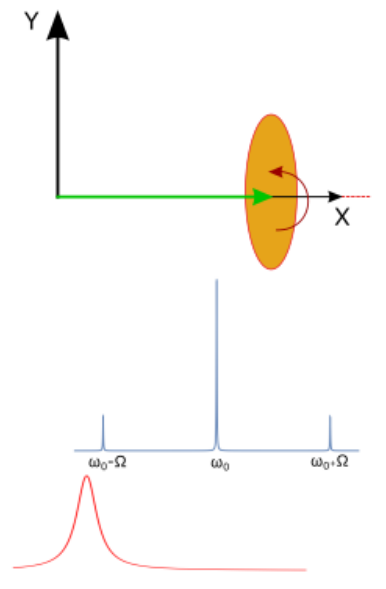

(a)

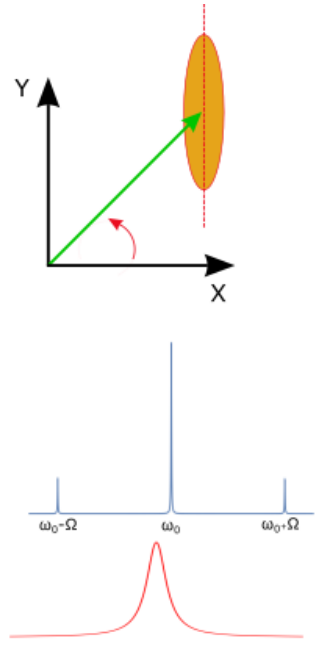

(b)

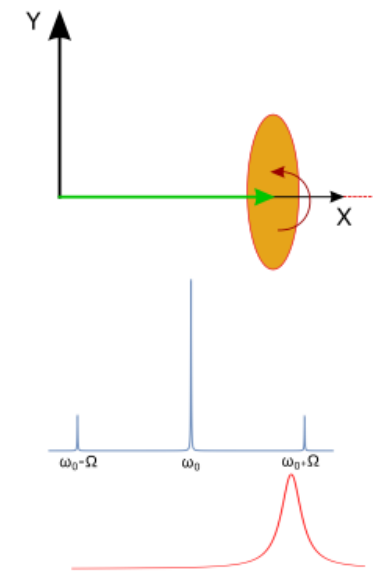

(c)

Figura 2.8: Cavidade de análise atuando na portadora e nas frequências de análise.

Agora que entendemos como a cavidade de análise atua na elipse de ruído podemos voltar ao caso mais geral e considerar termos dissipativos na nossa análise. Podemos reescrever a quadratura amplitude do campo refletido, $\hat{p}_{\Omega R}$, em função das quadraturas dos campos elétricos na entrada da cavidade $\hat{p}_{\Omega i n}$ e $\hat{q}_{\Omega i n}$ e do vácuo $\hat{p}_{\hat{b} \Omega R}$ e $\hat{q}_{\hat{b} \Omega R}$. Fazemos isso invertendo as equações 2.80, 2.81 e substituindo o resultado em 2.83. Como resultado, temos:

$$
\hat{p}_{\Omega R}=g_{p} \hat{p}_{\Omega i n}+i g_{q} \hat{q}_{\Omega i n}+g_{b p} \hat{p}_{\hat{b} \Omega}+i g_{b q} \hat{q}_{\hat{b} \Omega}
$$

onde os coeficientes $g_{p}$ e $g_{q}$ determinam de que forma a quadratura $\hat{p}_{\Omega R}$ do campo refletido depende do campo de entrada, e $g_{b p}$ e $g_{b q}$ determinam de que forma $\hat{p}_{\Omega R}$ depende do campo do vácuo. Os coeficientes $g_{p}, g_{q}, g_{b p}$ e $g_{b q}$ dependem implicitamente de $\Delta, \Delta_{ \pm \Omega}$. Tal dependência 
é omitida de 2.87 por questão de simplicidade e sua forma geral é dada por:

$$
\begin{aligned}
g_{p} & =\frac{1}{2}\left[e^{-i \phi_{R}(\Delta)} r\left(\Delta_{\Omega}\right)+e^{i \phi_{R}(\Delta)} r^{*}\left(\Delta_{-\Omega}\right)\right], \\
g_{q} & =\frac{1}{2}\left[e^{-i \phi_{R}(\Delta)} r\left(\Delta_{\Omega}\right)-e^{i \phi_{R}(\Delta)} r^{*}\left(\Delta_{-\Omega}\right)\right], \\
g_{v p} & =\frac{1}{2}\left[e^{-i \phi_{R}(\Delta)} t\left(\Delta_{\Omega}\right)+e^{i \phi_{R}(\Delta)} t^{*}\left(\Delta_{-\Omega}\right)\right], \\
g_{v q} & =\frac{1}{2}\left[e^{-i \phi_{R}(\Delta)} t\left(\Delta_{\Omega}\right)-e^{i \phi_{R}(\Delta)} t^{*}\left(\Delta_{-\Omega}\right)\right] .
\end{aligned}
$$

Variando o comprimento da cavidade, as dessintonias $\Delta, \Delta_{ \pm \Omega}$ também variam e com isso a cavidade de análise varre as frequências da portadora e das bandas laterais provocando a rotação da elipse de ruído. A fase da quadratura $\hat{p}_{\Omega R}$ depende dos coeficientes dados por 2.88 e, portanto, a fase depende de $\Delta$ e $\Delta_{ \pm \Omega}$ de forma que no processo de varrer essas dessintonias varremos todas as quadraturas do campo elétrico.

Lembrando que a fotocorrente se relaciona às quadraturas do campo elétrico por 2.50, podemos reescrever o espectro de ruído dado pela 2.56 em função das quadraturas generalizadas correspondentes. No caso do campo refletido pela cavidade de referência o espectro de ruído $S_{\Omega R}$ é dado por:

$$
S_{\Omega R} \delta\left(\Omega-\Omega^{\prime}\right)=\left\langle\hat{p}_{\Omega R} \hat{p}_{\Omega^{\prime} R}\right\rangle
$$

Substituindo 2.87 na equação 2.89 e considerando a condição $\Omega=\Omega^{\prime}$, encontramos a expressão que descreve o espectro de ruído $S_{\Omega R}$ :

$$
S_{\Omega^{\prime} R}=\left|g_{p}\right|^{2} S_{p_{i n}}+\left|g_{q}\right|^{2} S_{q_{i n}}+\left|g_{v p}\right|^{2}+\left|g_{v q}\right|^{2} .
$$

Ajustando a curva correspondente a equação 2.90 aos dados experimentais do espectro de ruído iremos obter os valores de $S_{p i n}$ e $S_{q i n}$. Sendo $S_{\hat{X}_{\varphi}}^{\text {vac }}(\Omega)=1$ o ruído quântico padrão. Caso $S_{\text {pin }}>1 \mathrm{e} / \mathrm{ou} S_{\text {qin }}>1$ apresentem excesso de ruído a quadratura correspondente a essa amplitude apresentará excesso de ruído, caso $S_{\text {pin }}<1$ ou $S_{\text {qin }}<1$ a quadratura correspondente apresenta compressão de ruído e caso $S_{\text {pin }}=S_{\text {qin }}$ estamos lidando com um estado coerente. A figura 2.9 apresenta a curva esperada para esses três casos. 


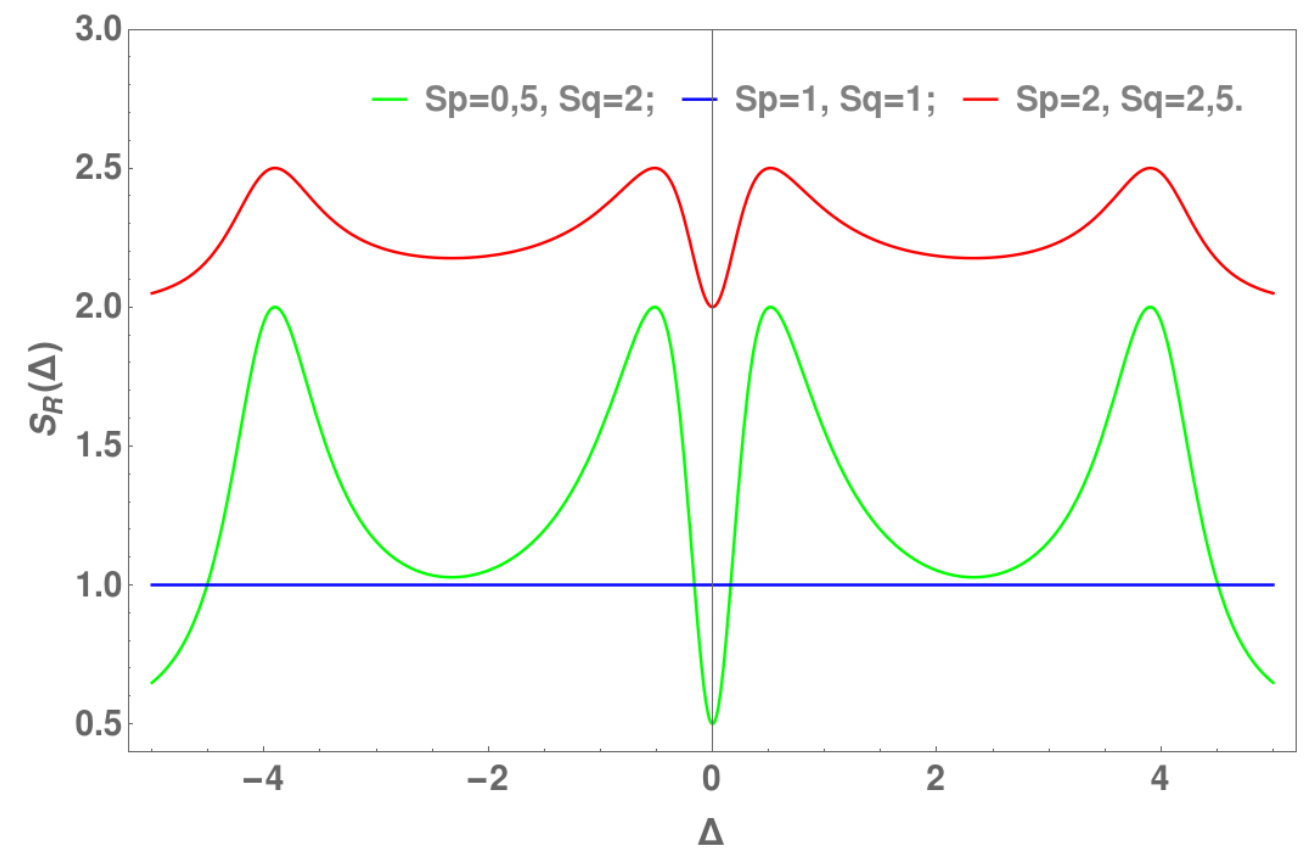

Figura 2.9: Espectro de ruído considerando: $S_{p}=0,5, S_{q}=2$ em verde, $S_{p}=1, S_{q}=1 \mathrm{em}$ azul e $S_{p}=2, S_{q}=2,5$ em vermelho. Em todos os casos consideramos $R_{\text {min }}=1$.

\subsection{Emaranhamento}

O emaranhamento é um fenômeno intrinsecamente quântico cuja discussão teve origem no famoso trabalho de Einstein, Podolsky e Rosen (EPR)[43]. O trabalho entitulado "Can Quantum Mechanics Description of Physical Reality be Considered Complete?" discute um experimento mental em que conhecemos a distância relativa $x_{1}-x_{2}=L$ e o momento total $p_{1}+p_{2}=0$ entre duas partículas (1 e 2) após a interação entre elas e um posterior afastamento. Fazendo-se medidas de posição ou momento da partícula 1 e em seguida, medidas da partícula 2 na mesma base, encontramos grandes correlações entre as medidas das duas partículas. Se aceitarmos que as medidas realizadas na partícula 1 não interferem no resultado das medidas realizadas na partícula 2, concluímos que as duas partículas deveriam ter posição e momento bem definidos para justificar a alta correlação, o que viola o princípio da incerteza. Caso o princípio da incerteza não fosse violado, deveria existir uma comunicação instantânea entre as duas partículas, o que não é permitido pela teoria da relatividade. Essa aparente inconsistência da mecânica quântica ficou conhecida como paradoxo EPR.

Para dar seguimento as ideias desenvolvidas no trabalho, eles definiram o conceito de realidade física e estabeleceram um critério suficiente, porém não necessário para determinar o que é um elemento de realidade física: Se, sem de forma alguma perturbar um sistema, pudermos prever com certeza (probabilidade igual a um) o valor de uma quantidade física, então existe um elemento da realidade física correspondente a essa quantidade física. Além 
disso, estabeleceram que para termos uma teoria completa é necessário que cada elemento da realidade física (sendo esses elementos determinados por meio de experimentos e medidas) possua uma contraparte na teoria.

Para explicar o resultado do experimento mental o trabalho propõe duas alternativas: (1) A descrição da realidade dada pela função de onda da mecânica quântica não é completa ou (2) quando dois operadores correspondentes a duas quantidades físicas não comutam as duas quantidades não têm realidade simultânea. A negação da alternativa (1) os leva a negação da alternativa (2), que consideram a outra única possível explicação para o paradoxo. Assim, o trabalho conclui que as grandezas posição e momento possuem realidade simultânea, no entanto, a mecânica quântica falha em prever simultaneamente resultados de observáveis conjugados e portanto seria incompleta.

No mesmo ano em que esse trabalho foi publicado, Bohr escreveu um artigo com o mesmo título em resposta ao paradoxo EPR [44]. A ideia fundamental desse artigo consiste no questionamento da definição da realidade física dada pelo EPR [43]. Mais especificamente, sobre a possibilidade de realizar medidas em sistemas quânticos sem perturba-los.

Se a hipótese de que a mecânica quântica é uma teoria incompleta estivesse correta, deveriam existir variáveis ocultas não acessíveis por meio dos experimentos da época responsáveis por tornar a mecânica quântica probabilística. No entanto o trabalho de Bell [45] fornece um teste para determinar se uma determinada classe de estados pode ou não ser descrita pela teoria de variáveis ocultas locais. Por meio desse teste foi mostrado que experimentos envolvendo medidas de polarização e spin de sistemas que hoje são ditos emaranhados não podem ser explicados por uma estatística de variáveis ocultas locais.

Quando a teoria de variáveis ocultas é descartada, conclui-se que é preciso abrir mão do conceito de realidade local. Atualmente, a conclusão aceita para o paradoxo do EPR é que para determinados sistemas não é possível descrever suas partes de forma independente, tais sistemas são ditos emaranhados. De maneira quantitativa, o estado do sistema está emaranhado quando não pode ser escrito como o produto tensorial de suas partes:

$$
\hat{\rho}=\sum w_{A} \hat{\rho}_{A}^{1} \otimes \hat{\rho}_{A}^{2}
$$

onde $\hat{\rho}_{A}^{1}$ e $\hat{\rho}_{A}^{2}$ representam as matrizes densidade dos subsistemas. No entanto, não é uma tarefa fácil demonstrar matematicamente que uma dada matriz $\hat{\rho}$ não pode ser escrita na forma da equação 2.91, porém, várias relações que implicam em emaranhamento foram estudas dando origem a critérios para identificar estados emaranhados. Nas próximas seções serão apresentados o critério de Duan e critério de positividade sob transposição parcial para determinar se subsistemas estão emaranhados. 


\subsubsection{Critério de Duan}

Nessa seção descrevemos o teste de Duan [46] que, em variáveis contínuas, é um critério de separabilidade suficiente para determinar emaranhamento entre duas partes de um subsistema de variáveis contínuas.

Consideremos um estado genérico descrito pelo operador $\hat{\rho}$ e os operadores tipo EPR, que são descritos por:

$$
\begin{aligned}
& \hat{u}=|a| \hat{q}_{1}+\frac{1}{a} \hat{q}_{2}, \\
& \hat{v}=|a| \hat{p}_{1}-\frac{1}{a} \hat{p}_{2},
\end{aligned}
$$

onde os operadores $\hat{p}$ e $\hat{q}$ obedecem a relação de comutação $[\hat{p}, \hat{q}]=i \delta_{i, j}$ para $i, j=1,2$. O estado descrito por $\hat{\rho}$ está emaranhado se viola a desigualdade:

$$
\left\langle(\Delta \hat{u})^{2}\right\rangle+\left\langle(\Delta \hat{v})^{2}\right\rangle \geq a^{2}+\frac{1}{a^{2}}
$$

onde $a$ é um número real arbitrário diferente de zero.

\subsubsection{Critério de positividade sob transposição parcial (PPT)}

Nessa seção apresentaremos o critério de positividade sob transposição parcial (PPT) para o caso de variáveis contínuas. Tal critério foi elaborado por Peres [47] para sistemas bipartites discretos e posteriormente estendido a variáveis contínuas por Simon [48]. De forma geral, esse critério é suficiente para determinar se dois sistemas estão emaranhados. No entanto, para o caso de estados gaussianos, o critério é necessário e suficiente.

Sendo $\overrightarrow{\mathbf{X}}$ o vetor que descreve os momentos de primeira ordem de um dado sistema, podemos descrever as relações de comutação entre seus elementos na forma compacta conhecida como forma simplética:

$$
\left[\hat{X}_{\alpha}, \hat{X}_{\beta}\right]=2 i \Omega_{\alpha \beta}, \quad \alpha, \beta=1,2,3,4, \ldots
$$

onde:

$$
\Omega=\bigoplus_{i=1}^{N} J \text { e } J=\left(\begin{array}{cc}
0 & 1 \\
-1 & 0
\end{array}\right)
$$

Sendo os elementos da matriz de covariância $\mathbf{V}$ dados por:

$$
V_{\alpha \beta}=\frac{1}{2}\left\langle\left\{\delta \hat{X}_{\alpha}, \delta \hat{X}_{\beta}\right\}\right\rangle,
$$


o princípio da incerteza pode ser enunciado como:

$$
V+i \Omega \geq 0
$$

A ideia central por traz do critério (PPT) é que se a matriz $\rho$ é composta por dois subsistemas separáveis, $\hat{\rho}=\hat{\rho}^{1} \otimes \hat{\rho}^{2}$, a transposição parcial de uma das partes do sistema de variáveis contínuas equivale a uma reflexão especular de uma das variáveis canônicas do subsistema transposto. Sendo assim, a matriz obtida pela transposição parcial de uma das partes da matriz $\hat{\rho}, \hat{\rho}^{\prime}=\hat{\rho}^{\tau_{1}}$ ou $\hat{\rho}^{\prime}=\hat{\rho}^{\tau_{2}}$, ainda deve representar um sistema físico. Portanto, $\rho$ deve obedecer o princípio da incerteza que em termos de sua matriz de covariância, $\tilde{V}$, deve satisfazer à desigualdade:

$$
\tilde{V}+i \Omega \geq 0
$$

Nesse texto, iremos nos limitar a apresentar a forma prática de aplicar o critério PPT que está descrita em [49].

Para um sistema cujo vetor de variáveis canônicas é dado por $\hat{X}=\left(\hat{p}_{1}, \hat{q}_{1}, \hat{p}_{2}, \hat{q}_{2}\right)^{T}$ a transposição parcial da matriz de covariância é implementada por meio da transformação:

$$
\tilde{V}=\Upsilon V \Upsilon, \text { onde } \Upsilon=\operatorname{diag}(1,1,1,-1)
$$

Após encontrar $\tilde{V}$ calcula-se os autovalores $o_{k}$ da matriz $\nu=-\left(\tilde{V} \Omega^{2}\right)$. Os autovalores simpléticos da matriz $\tilde{V}$, são dados por $\nu_{k}=\sqrt{o_{k}}$. Se algum dos autovalores simpléticos $\nu_{k}<1$, então o sistema transposto se encontra emaranhado com o sistema restante.

\subsection{Mistura de quatro ondas}

A mistura de quatro ondas (do inglês Four Wave Mixing - 4WM) é um dos possíveis processos paramétricos $\chi^{(3)}$ que podem ocorrer quando há a interação entre um campo eletromagnético intenso, como o campo de bombeio desse experimento, e um meio atômico. De forma geral, os processos paramétricos ocorrem quando um campo elétrico $\mathbf{E}(\mathbf{r}, t)$ incide em um meio não linear, dando origem a uma polarização $\mathbf{P}(\mathbf{r}, t)$ dependente de termos de ordem mais alta do campo, sendo cada uma de suas componentes dadas por [50]:

$$
\begin{aligned}
P_{k}= & \epsilon_{0} \sum_{n} \sum_{i} \chi_{i k}^{(1)} E_{i}\left(\omega_{n}\right)+\epsilon_{0} \sum_{m n} \sum_{i j} \chi_{i j k}^{(2)} E_{i}\left(\omega_{m}\right) E_{j}\left(\omega_{n}\right) \\
& +\epsilon_{0} \sum_{m n o} \sum_{i j k} \chi_{i j l k}^{(3)} E_{i}\left(\omega_{m}\right) E_{j}\left(\omega_{n}\right) E_{l}\left(\omega_{o}\right)+\ldots,
\end{aligned}
$$

onde os índices $i, j, k$ se referem a componentes cartesianas e $m, n, o$ as componentes em frequência do campo $\mathbf{E}(\mathbf{r}, t)$. As dependências em $\mathbf{r}$ e $t$ da polarização e do campo elétrico foram 
omitidas por simplicidade.

Para meios atômicos, despreza-se o termo $\chi^{(2)}$, pois esse é nulo para materiais centrosimétricos, e os fenômenos de interesse ocorrerão devido ao termo $\chi^{(3)}$. Devido à geometria do experimento, o único processo paramétrico de relevância será o 4WM.

(a)

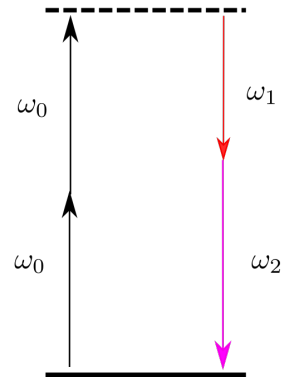

(b)

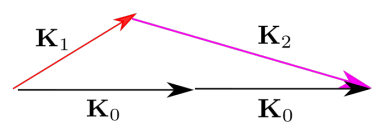

Figura 2.10: (a) Conservação de energia no processo de 4WM: $2 \omega_{0}=\omega_{1}+\omega_{2}$. (b) Conservação de momento no processo de $4 \mathrm{WM}: 2 \mathbf{k}_{0}=\mathbf{k}_{1}+\mathbf{k}_{2}$.

O processo de criação e aniquilação de fótons deve satisfazer à conservação de energia, também conhecida como acordo de frequência, ilustrada na figura 2.10 (a). A energia de um fóton de frequência $\omega_{i}$ é dada por $E=\hbar \omega_{i}$. Dessa forma, a soma das frequências dos fótons aniquilados deve ser igual a soma das frequências dos fótons produzidos,

$$
2 \omega_{0}=\omega_{1}+\omega_{2}
$$

Além disso, deve haver conservação do momento, também conhecido como casamento de fase, ilustrado na figura 2.10 (b). Sendo os vetores de onda dos campos em questão dados por $\mathbf{k}_{i}$, a condição

$$
2 \mathbf{k}_{0}=\mathbf{k}_{1}+\mathbf{k}_{2}
$$

deve ser satisfeita para que haja casamento de fase.

Uma das possíveis formas do 4WM é ilustrada na figura 2.11. Nessa figura podemos ver dois feixes atravessando um meio atômico, o feixe de bombeio (em preto) e o feixe de prova (em vermelho). O feixe de bombeio intenso provoca polarização no meio atômico, que possui uma não linearidade $\chi^{3}$. O feixe de prova, de pouca intensidade, serve apenas como semente. A interação entre o feixe de bombeio e o feixe de prova no interior do meio atômico tem como efeito transferir energia do feixe de bombeio para o feixe de prova e feixe conjugado (em roxo).

Devido à sua geometria, dizemos que o diagrama de energia do processo de mistura de quatro ondas possui a configuração de duplo- $\Lambda$, assim como ilustrado na figura Figura 2.12(b), no qual vemos a aniquilação de dois fótons do bombeio com a produção de dois fótons nos modos de prova e conjugado. Na mecânica quântica a mistura de quatro ondas pode ser descrita pelo 


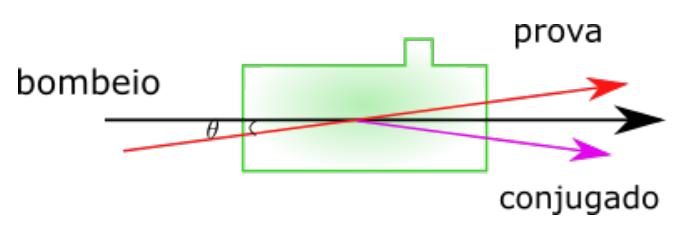

Figura 2.11: geometria do experimento: Feixe de bombeio (em preto) e feixe de prova (em vermelho) atravessam a célula de vapor atômico formando um pequeno ângulo $\theta$ entre si. $\mathrm{A}$ mistura de quatro ondas dá origem a um feixe conjugado (em roxo) e intensifica o feixe de prova.

Hamiltoniano:

$$
\hat{\mathscr{H}}=i \hbar \beta \hat{a}_{0}^{2} \hat{a}_{2} \hat{a}_{1}+h . c . .
$$

Onde, $\beta$ é o parâmetro da força da interação, $\hat{a}_{0}, \hat{a}_{1}$ e $\hat{a}_{2}$ são os operadores de aniquilação dos fótons dos feixes de bombeio, prova e conjugado, respectivamente.

No experimento desse trabalho utilizamos uma célula de átomos de rubídio como meio de ganho do processo de 4WM e dessintonizamos o campo de bombeio $800 \mathrm{MHz}$ acima da transição $5 S_{1 / 2} \mathrm{~F}=2 \rightarrow 5 P_{1 / 2}$ da linha $\mathrm{D} 1$ do rubídio, cujo diagrama de níveis completo é ilustrado na figura 2.12 (a). Existem duas configurações de 4WM diferentes que merecem nossa atenção: A primeira é o 4WM não degenerado, na qual os campos de prova e conjugado encontram-se dessintonizados da frequência do bombeio. Como escolhemos trabalhar com a transição $5 S_{1 / 2}$, $\mathrm{F}=2 \rightarrow 5 P_{1 / 2}$ da linha $\mathrm{D} 1$ do rubídio, isso significa que os campos de prova e conjugado estão dessintonizados de $\pm 3 \mathrm{GHz}$ da frequência do bombeio, pois essa é a diferença entre as energias da linha $5 S_{1 / 2} \mathrm{~F}=2$ e $5 S_{1 / 2} \mathrm{~F}=3$, como ilustrado na figura 2.12 (b). A segunda configuração é o 4WM degenerado,na qual a multiplicidade de níveis zeeman do nível $\mathrm{F}=2$ possibilita uma multiplicidade de transições $\lambda$, e nesse caso, os campos de prova e conjugado são produzidos aproximadamente na mesma frequência que o bombeio, assim como ilustrado na figura 2.12 (c).

A mistura de quatro ondas pode ser utilizada como fonte de estados emaranhados do campo e possui interessantes aplicações na área de ótica quântica. Recentemente, foram feitos muitos experimentos de 4WM em células de vapor de rubídio demonstrando compressão de ruído e emaranhamento entre os feixes desse sistema. Glorieux et al. [20] observaram grandes quantidades de correlações não clássicas, e.g., compressão na quadratura das diferenças nas intensidades entre os feixes de prova e conjugado produzidos por 4WM com bombeio próximo à ressonância com a linha D1 do Rubídio 85 em função da temperatura, dessintonias do feixe de bombeio etc. Pooser et al. [21] realizaram uma importante caracterização de alguns parâmetros do 4WM, destacando-se a compressão no ruído da diferença nas intensidades dos feixes de prova e conjugado em função da dessintonia para as linhas D1 e D2 do Rubídio 85 e 87. Vincent Boyer et al. [3] utilizaram um amplificador multi-modo baseado em 4WM para produzir imagens gêmeas que exibem emaranhamento localizado, e esse trabalho demonstra que o sistema utilizado é 
(a)

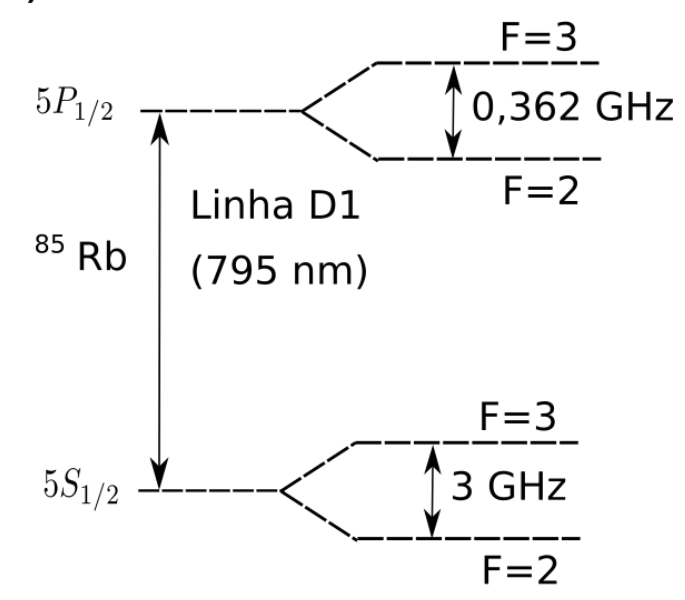

(b)

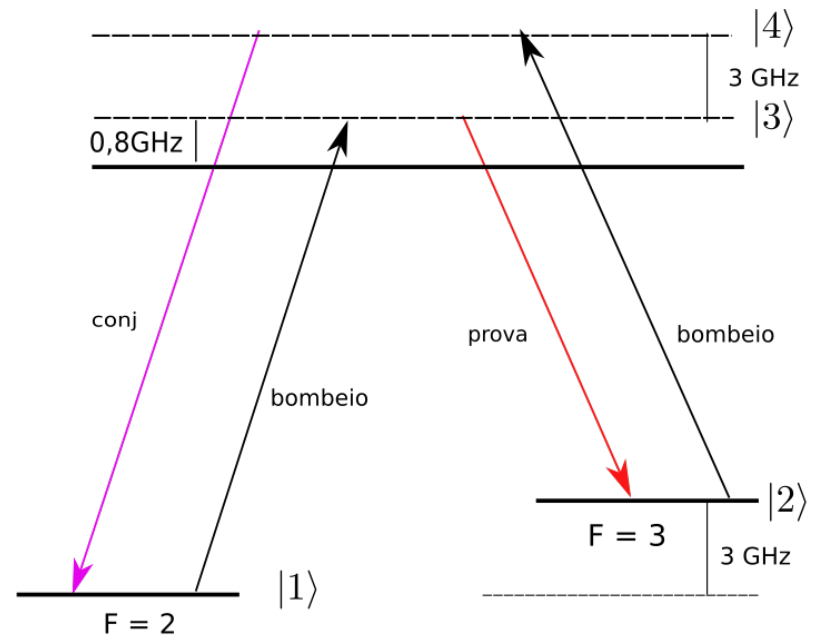

(c)

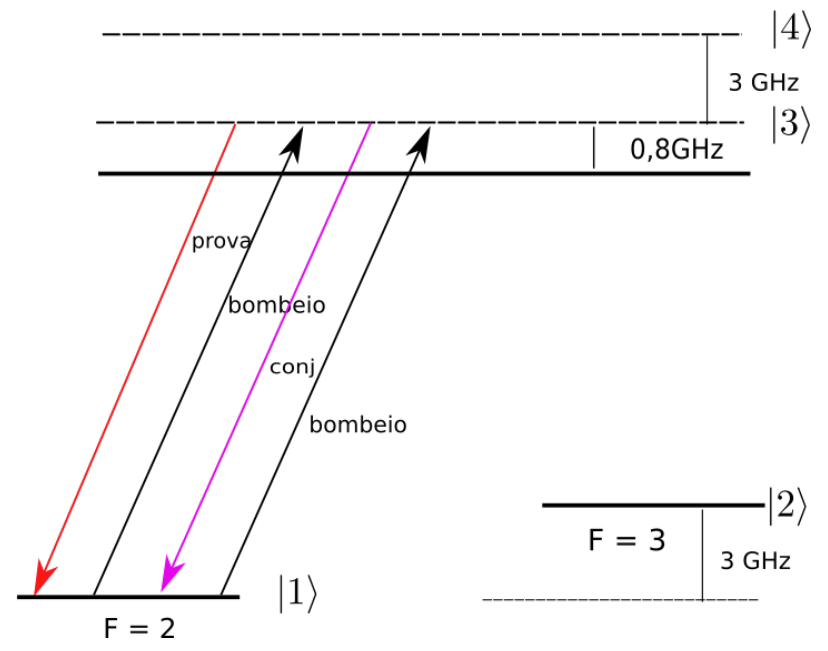

\section{)} $3\rangle$

Figura 2.12: Parte (a): Níveis hiperfinos da linha D1 do Rubídio 85. Parte (b): Diagrama dos níveis de energia da mistura de quatro ondas não degenerada. Átomo e campo de bombeio se acoplam ao campo de prova permitindo a primeira transição $\lambda$, um segundo acoplamento entre o átomo e o campo de bombeio se acoplam ao campo conjugado fechando a segunda transição $\lambda$. Parte (c): Diagrama dos níveis de energia da mistura de quatro ondas degenerada. Nesse caso também temos duas transições $\lambda$ sendo fechadas devido ao acoplamento entre o átomo e o campo de bombeio e os campos de prova e conjugado. No entanto, no caso degenerado as transições $\lambda$ ocorrem devido aos níveis zeeman de $\mathrm{F}=2$ e nesse caso, prova e conjugado são produzidos praticamente na mesma frequência do bombeio.

uma fonte ideal para produção de protocolos de informação quântica em variáveis contínuas.

Os principais conceitos relacionados a cavidades óticas e a mistura de quatro ondas foram introduzidos nesse capítulo. Dessa forma, temos a base teórica para fazer a descrição do OPO (oscilador paramétrico ótico). Sendo assim, apresentaremos no capítulo que se segue [Capítulo 3] o estudo teórico do OPO $\chi^{3}$ que foi desenvolvido nesse trabalho. 


\section{Capítulo 3}

\section{Estudo teórico do OPO com meio de ganho não linear de terceira ordem}

Nesse capítulo utilizamos os conceitos desenvolvidos no capítulo 2 para explicar os fundamentos do oscilador paramétrico ótico (OPO) que é introduzido na seção 3.1 e como base para o desenvolvimento do modelo teórico do OPO $\chi^{3}$. Na seção 3.2 fazemos um estudo dos ganhos e depleções que os campos envolvidos no processo de $4 \mathrm{WM}$ sofrem ao se propagarem pelo meio de ganho. Na seção 3.3 e 3.4 estudamos o efeito que a realimentação produzida pelas cavidades do DROPO e TROPO $\chi^{3}$ introduzem ao sistema e determinaremos os valores médios dos campos de saída dos feixes de bombeio, prova e conjugado para cada tipo de cavidade. Na seção 3.5 reescrevemos o hamiltoniano do 4WM utilizando operadores de criação e aniquilação de fótons expandidos em termos da frequência das bandas laterais e, dessa forma, encontramos o hamiltoniano do 4WM no domínio da frequência. Por meio desse hamiltoniano no domínio da frequência, estudamos as flutuações das amplitudes dos campos envolvidos no 4WM. Em seguida estudamos o efeito da cavidade sobre as flutuações destas amplitudes [seção 3.6]. Como estamos considerando a aproximação de estados Gaussianos, essas flutuações são determinadas pela matriz de covariância calculada na subseção 3.6.1. As seções que se seguem fazem um estudo detalhado das variâncias e correlações entre os modos dos campos de bombeio, prova e conjugado dos sistemas DROPO e TROPO $\chi^{3}$. Além disso, fazemos uma caracterização das regiões de emaranhamento do sistema por meio dos testes de Duan e PPT, destacamos o estudo feito por meio do critério PPT nas seções 3.9 para o DROPO e para 3.12 o TROPO.

\subsection{Oscilador Paramétrico Ótico}

O oscilador paramétrico ótico, OPO, consiste de uma fonte de luz que incide em uma cavidade ótica com um meio de ganho não linear em seu interior. Na Figura 3.1 vemos uma de suas possíveis configurações, o OPO duplamente ressonante com meio de ganho $\chi^{(3)}$ cuja cavidade 
apresenta geometria triangular. A figura mostra um campo de frequência $\omega_{0}$ que incide em um meio não linear de terceira ordem e gera dois outros campos de frequências $\omega_{1}$ e $\omega_{2}$. O campo de bombeio e os campos gerados possuem sobreposição espacial, no entanto, por razões didáticas, a trajetória de cada um dos campos é apresentada separadamente. Manteremos essa apresentação didática nas demais cavidades apresentadas nessa tese. Devido a sua polarização vertical, o feixe de frequência $\omega_{0}$ faz uma passagem simples pelo meio de ganho, ou seja, ele incide no primeiro PBS e é totalmente refletido, se propaga livremente pelo meio de ganho e, em seguida, é refletido para fora da cavidade pelo segundo PBS. Denominamos essa propagação livre do bombeio pela célula de rubídio de passagem simples. Os feixes gerados, $\omega_{1}$ e $\omega_{2}$, possuem polarização horizontal, de forma que sua trajetória não é afetada pelos PBSs. Os campos gerados são totalmente refletidos pelos espelhos $R_{1}$ e $R_{2}$ e parcialmente refletidos pelo espelho de reflectância $R$, de forma que uma fração desses campos é transmitida para fora da cavidade e a fração restante permanece aprisionada. Dizemos que essa fração do campo que permanece aprisionada na cavidade está cavitando.

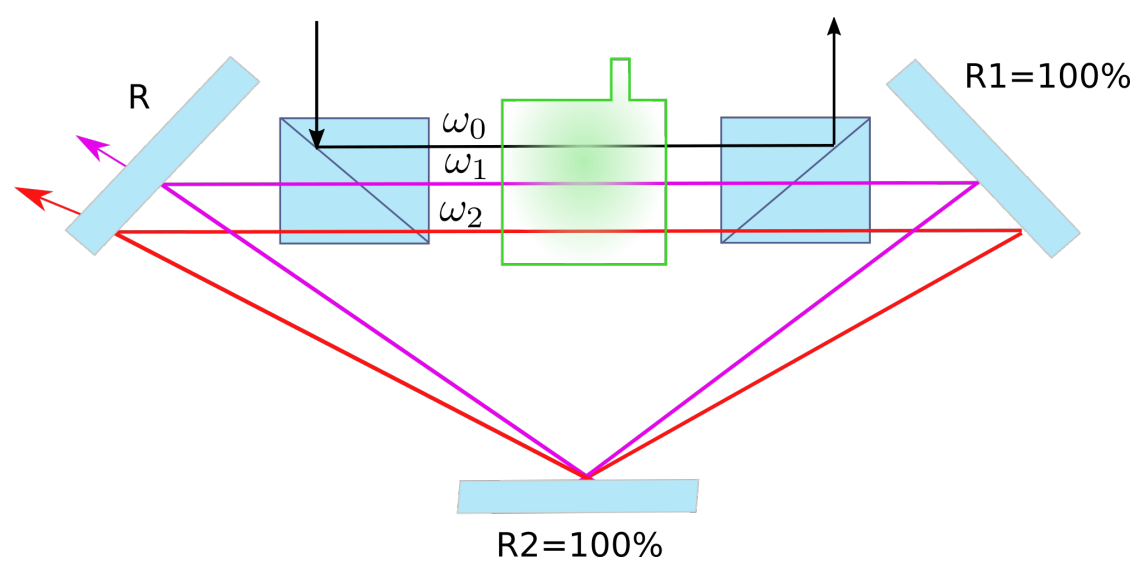

Figura 3.1: OPO duplamente ressonante: Cavidade ótica triangular formada por dois espelhos de reflectividade de $R_{1}=R_{2}=100 \%$ com dois PBSs em seu interior. O espelho $R$ é parcialmente refletor para os campos $\omega_{1}$ e $\omega_{2}$. Um laser de frequência $\omega_{0}$ e polarização vertical faz uma passagem simples por um meio não linear de terceira ordem e gera dois outros feixes de frequências $\omega_{1}$ e $\omega_{2}$ com polarização vertical. O feixe de frequência $\omega_{0}$ faz uma passagem simples pelo meio de ganho. Os feixes criados, $\omega_{1}$ e $\omega_{2}$ passam a cavitar.

Nesse trabalho, o meio de ganho será uma célula de vapor de rubídio. Da interação entre a luz e os átomos de rubídio ocorre o fenômeno de 4WM descrito na seção 2.14, ou seja, acontece a aniquilação de dois fótons do bombeio de frequência $\omega_{0}$ e criação de um fóton no modo de prova, $\omega_{1}$, e um fóton no modo conjugado, $\omega_{2}$.

$\mathrm{O}$ OPO possui um limiar de oscilação referente à potência do feixe de bombeio, quando essa potência é tal que os ganhos dos feixes de prova e conjugado superam as perdas (úteis e espúrias) a cavidade passa a emitir feixes de intensidade média não nula.

Consideramos o campo de entrada do bombeio na cavidade do OPO coerente, enquanto os 
campos de entrada dos modos de prova e conjugado são campos de vácuo. Tanto o estado coerente como o estado de vácuo são estados gaussianos, ou seja, são completamente caracterizados por seus momentos de primeira e segunda ordem. Tais campos irão sofrer a evolução temporal dada pelo Hamiltoniano: $\hat{H}=i \hbar \beta \hat{a}_{0}^{2} \hat{a}_{1}^{\dagger} \hat{a}_{2}^{\dagger}+h . c$. . Fazendo a linearização do campo elétrico, tal como descrita pela equação 2.38, podemos reescrever o Hamiltoniano da mistura de quatro ondas como $\hat{H}=i \hbar \beta\left(\alpha_{0}+\delta \hat{a}_{0}\right)^{2}\left(\alpha_{1}^{*}+\delta \hat{a}_{1}^{\dagger}\right)\left(\alpha_{2}^{*}+\delta \hat{a}_{2}^{\dagger}\right)+h . c$. . Com essa informação podemos fazer uma análise sobre os campos de saída do OPO nas diferentes regiões de funcionamento do sistema, abaixo do limiar, na região do limiar e acima do limiar de oscilação.

Para um OPO que opera abaixo do limiar de oscilação o campo do bombeio não sofre depleção podendo ser considerado um campo clássico, $\alpha_{0}$, e os campos de prova e conjugado possuem valor médio nulo, portanto podem ser reescritos como $\delta \hat{a}_{1}$ e $\delta \hat{a}_{2}$. Sendo assim, abaixo do limiar, podemos expressar o Hamiltoniano da mistura de quatro ondas como $\hat{H}=i \hbar \beta \alpha_{0}^{2} \delta \hat{a}_{1}^{\dagger} \delta \hat{a}_{2}^{\dagger}+h . c$. Os campos resultantes da evolução temporal de um estado gaussiano por um Hamiltoniano bilinear sempre serão estados gaussianos. Dessa forma, os campos de saída do OPO abaixo do limiar serão gaussianos.

Para um OPO que opera acima do limiar ocorre a depleção do bombeio e emissão de campos de intensidade média não nula para os campos de prova e conjugado, pouco acima do limiar já podemos considerar que $\alpha \gg \delta \hat{a}$. Nesse caso, desprezamos flutuações de ordem superior a 2 na expansão do Hamiltoniano. Como resultado, temos um Hamiltoniano que ainda é bilinear, e cuja atuação em um estado gaussiano irá produzir outro estado gaussiano.

No caso de um OPO que opera em uma região muito próxima ao limiar de oscilação temos os valores médios dos campos comparáveis às flutuações dos mesmos, ou seja, $\alpha \approx \delta \hat{a}$. Nesse caso, o estudo deve utilizar um Hamiltoniano em que as flutuações de terceira ordem ou superiores contribuam para a evolução do estado dos campos podem gerar estados não gaussianos a partir de estados gaussianos. Sendo assim, a região do limiar do OPO é uma fonte interessante de estados não gaussianos.

A configuração mais utilizada em experimentos envolvendo OPOs é o OPO $\chi^{2}$, cujo meio de ganho é um meio não linear de segunda ordem. No caso do OPO $\chi^{2}$, quando o bombeio atinge o meio de ganho, ocorre a conversão paramétrica descendente dada pelo Hamiltoniano: $\hat{H}_{\chi^{2}}=i \hbar \beta \hat{a}_{0} \hat{a}_{1}^{\dagger} \hat{a}_{2}^{\dagger}+$ h.c., em que $\hat{a}_{0}$ se refere ao campo do bombeio, $\hat{a}_{1}$ ao campo sinal e $\hat{a}_{2}$ ao campo complementar. No entanto, nosso experimento utiliza o OPO $\chi^{3} 3$ pois não é uma tarefa fácil estudar o limiar de oscilação do OPO $\chi^{2}$. Nesses experimentos, necessitamos de cavidades com espelhos de baixa transmissão devido ao baixo ganho dos campos sinal e complementar. Tipicamente, utilizam-se cavidades de transmissão torno de 4\%. Nesse caso, a transição pelo limiar de oscilação é abrupta o que torna o estudo do limiar de oscilação mais difícil. Para um meio de ganho não linear de terceira ordem, $\chi^{3}$, temos altos ganhos dos campos de prova e conjugado, superiores a $100 \%$. Isso possibilita a utilização de cavidades com espelhos de maior transmissão e, assim, a transição pelo limiar de oscilação é mais suave. 
O objetivo do projeto no qual esse trabalho está inserido é utilizar o OPO com meio de ganho $\chi^{3}$ para estudar o limiar de oscilação do OPO. Esperamos que o experimento desse projeto produza estados não gaussianos, que merecem nossa atenção por possibilitar capacidade de processamento de informação além da que pode ser obtida por meio de estados gaussianos.

\subsection{Ganho dos campos de prova e conjugado}

O estudo feito nessa seção tem por objetivo determinar o ganho do 4WM (potência do feixe de bombeio que é convertida nos feixes de prova e conjugado durante sua propagação pelo meio não linear de ordem três $\left(\chi^{3}\right)$ ) em uma passagem simples do feixe de bombeio pelo meio de ganho. Para o experimento em estudo o meio de ganho é a célula de rubídio. Para determinar a dinâmica do sistema consideramos o Hamiltoniano de interação do 4WM dado por:

$$
\hat{H}_{\chi}=i \hbar \beta \hat{a}_{0}^{2} \hat{a}_{1}^{\dagger} \hat{a}_{2}^{\dagger}+h . c . .
$$

Para determinar as amplitudes médias dos campos de saída dos feixes de bombeio, prova e conjugado em função dos parâmetros do sistema na saída do OPO é preciso conhecer o ganho na propagação desses campos pela célula de rubídio. Por meio das equações de Heisenberg obtemos a evolução dos operadores $\hat{a}_{n} \operatorname{com} n=0,1,2$ na propagação pelo meio de ganho:

$$
\frac{d}{d t} \hat{a}_{n}=\frac{i}{\hbar}\left[H_{\chi}, \hat{a}_{n}\right]
$$

Como resultado das equações de Heisenberg obtemos as equações diferenciais:

$$
\begin{aligned}
\frac{d}{d t} \hat{a}_{0} & =-2 \beta \hat{a}_{0}^{\dagger} \hat{a}_{1} \hat{a}_{2}, \\
\frac{d}{d t} \hat{a}_{1} & =\beta \hat{a}_{0}^{2} \hat{a}_{2}^{\dagger}, \\
\frac{d}{d t} \hat{a}_{2} & =\beta \hat{a}_{0}^{2} \hat{a}_{1}^{\dagger},
\end{aligned}
$$

onde $\hat{a}_{0}, \hat{a}_{1}, \hat{a}_{2}$ representam os operadores de aniquilação referentes aos campos dos feixes de bombeio, prova e conjugado, respectivamente. No estudo que segue o operador $\hat{a}$ será descrito em sua forma linearizada dada pela equação 2.38. Dessa forma, para determinar a evolução dos valores médios do campo faz-se a correspondência entre os operadores e as amplitudes 
complexas do campo elétrico, desprezando-se as flutuações $\delta \hat{a}$ :

$$
\begin{aligned}
\frac{d}{d t} \alpha_{0} & =-2 \beta \alpha_{0}^{*} \alpha_{1} \alpha_{2}, \\
\frac{d}{d t} \alpha_{1} & =\beta \alpha_{0}^{2} \alpha_{2}^{*}, \\
\frac{d}{d t} \alpha_{2} & =\beta \alpha_{0}^{2} \alpha_{1}^{*},
\end{aligned}
$$

onde $\alpha_{i}$, com $i=0,1,2$, são números complexos que representam as amplitudes dos campos referentes aos feixes de bombeio, prova e conjugado, respectivamente. Esses campos serão representados pelos números complexos $\alpha_{0}=\sqrt{P_{0}} e^{i \theta_{0}}, \alpha_{1}=\sqrt{P_{1}} e^{i \theta_{1}}, \alpha_{2}=\sqrt{P_{2}} e^{i \theta_{2}}$ em que $\sqrt{P_{i}}$ representa a amplitude e $\theta_{i}$ a fase do campo elétrico. Assim sendo, a potência do campo elétrico é associada a $P_{i}=\alpha_{i} \alpha_{i}^{*}$. Diferenciando $P_{i}$, encontramos:

$$
\frac{d P_{i}}{d t}=\frac{d \alpha_{i}}{d t} \alpha_{i}^{*}+\frac{d \alpha_{i}^{*}}{d t} \alpha_{i}
$$

Então, rescrevendo a equação 3.5 e substituindo as equações 3.4 para cada um dos três campos estudados, temos:

$$
\begin{aligned}
& \frac{d P_{0}}{d t}=\frac{d \alpha_{0}}{d t} \alpha_{0}^{*}+\frac{d \alpha_{0}^{*}}{d t} \alpha_{0}=-2 \beta\left(\alpha_{0}^{* 2} \alpha_{1} \alpha_{2}+\alpha_{0}^{2} \alpha_{1}^{*} \alpha_{2}^{*}\right), \\
& \frac{d P_{1}}{d t}=\frac{d \alpha_{1}}{d t} \alpha_{1}^{*}+\frac{d \alpha_{1}^{*}}{d t} \alpha_{1}=\beta\left(\alpha_{0}^{2} \alpha_{1}^{*} \alpha_{2}^{*}+\alpha_{0}^{* 2} \alpha_{1} \alpha_{2}\right), \\
& \frac{d P_{2}}{d t}=\frac{d \alpha_{2}}{d t} \alpha_{2}^{*}+\frac{d \alpha_{2}^{*}}{d t} \alpha_{2}=\beta\left(\alpha_{0}^{2} \alpha_{1}^{*} \alpha_{2}^{*}+\alpha_{0}^{* 2} \alpha_{1} \alpha_{2}\right) .
\end{aligned}
$$

Reescrevendo em termos de $P_{i}$ e $\theta=-2 \theta_{0}+\theta_{1}+\theta_{2}$ :

$$
\begin{aligned}
\frac{d P_{0}}{d t} & =-4 \beta \sqrt{P_{1} P_{2}} P_{0} \cos (\theta) \\
\frac{d P_{1}}{d t} & =2 \beta \sqrt{P_{1} P_{2}} P_{0} \cos (\theta) \\
\frac{d P_{2}}{d t} & =2 \beta \sqrt{P_{1} P_{2}} P_{0} \cos (\theta) .
\end{aligned}
$$

Para que o OPO esteja ressonante esperamos o angulo $\theta$ que maximize o acoplamento, portanto, consideraremos $\cos (\theta)=1$.

Seja $x$ a variável que representa o ganho em potência dos feixes de prova e conjugado ao atravessar o meio de ganho, temos:

$$
\begin{aligned}
& P_{0}(t)=P_{0}(0)-2 x, \\
& P_{1}(t)=P_{1}(0)+x, \\
& P_{2}(t)=P_{2}(0)+x,
\end{aligned}
$$


Sempre que o feixe de prova recebe um acréscimo de potência $x$ o feixe conjugado também ganha o mesmo acréscimo de potência e o feixe de bombeio, que está cedendo essa energia, sofre uma perda de potência de $2 x$. Sabendo que a semente do feixe de prova possui baixa intensidade (da ordem de $\mu W$ ) em relação a intensidade do feixe de bombeio (da ordem de dezenas ou centenas de $m W$ ) podemos dizer que os valores de $P_{1}(0)$ e $P_{2}(0)$ se devem a passagem pelo meio de ganho e a volta na cavidade que será tratada em detalhes nas próximas seções. Assim, na ausência de uma semente, podemos considerar $P_{1}(t)=P_{2}(t)$ e a equação 3.7 pode ser reescrita como:

$$
\frac{d x}{d t}=2 \beta\left(P_{0}(0)-2 x\right)\left(P_{1}(0)+x\right)
$$

Para determinar o ganho total do sistema, integramos a equação 3.9 entre os limites: $x=0$ na entrada do meio de ganho $(t=0)$ e $x=G \equiv \Delta P 1=P_{1}\left(L / V_{m}\right)-P_{1}(0)$ na saída do meio de ganho $\left(t=L / V_{m}\right)$, em que $L$ representa o comprimento da célula de rubídio e $V_{m}$ representa a velocidade da luz no interior da célula de rubídio, que é aproximadamente a velocidade da luz no vácuo. O resultado dessa integração pode ser expresso em termos da constante $\kappa=\beta L / V_{m}$ que é proporcional ao tempo de voo do fóton no interior do meio de ganho. A expressão que descreve o ganho do nosso sistema é dada por:

$$
G=\frac{P_{0}(0) P_{1}(0)\left(e^{2 \kappa\left(P_{0}(0)+2 P_{1}(0)\right)}-1\right)}{2 P_{1}(0) e^{2 \kappa\left(P_{0}(0)+2 P_{1}(0)\right)}+P_{0}(0)} .
$$

Quando não há saturação do bombeio, ou seja, a potência do bombeio é muito maior que a potência dos campos gerados, o ganho tende à $G=P_{1}(0) e^{2 \kappa\left(P_{0}(0)+2 P_{1}(0)\right)}$. Isso significa que o ganho em potência dos campos gerados é proporcional ao parâmetro $g=e^{2 \kappa\left(P_{0}(0)+2 P_{1}(0)\right)}$, o qual denominamos ganho não saturado do sistema.

O sistema não saturado corresponde a uma cavidade totalmente aberta (transmissão de $100 \%$ ) e com uma semente do campo de prova muito pequena quando comparada ao feixe de bombeio, $P_{1}(0) / P_{0}(0) \simeq 0$. Nessa situação temos:

$$
g=e^{2 \kappa P_{0}(0)}=\frac{\Delta P_{1}}{P_{1}(0)}+1
$$

Da equação 3.11 vemos que o ganho relativo $\frac{\Delta P_{1}}{P_{1}(0)}$ cresce exponencialmente com o aumento da potência de bombeio e com o aumento da constante $\kappa$ (aumento do tempo de interação com o meio). Também é interessante observar que esse ganho relativo é inversamente proporcional à potência da semente do feixe de prova.

Uma forma de estimar o valor da constante $\kappa$ é por meio de valores experimentais. Com base em artigos correlatos a [51] observamos que para uma potência de entrada $P_{0}(0)=0,3 W$ temos um ganho relativo $\frac{\Delta P_{1}}{P_{1}(0)}=6$. Substituindo os valores experimentais de $P_{0}(0)$ e do ganho relativo na equação 3.11 encontramos $\kappa=3 W^{-1}$. 
Até esse momento descrevemos o ganho do campo de prova pela equação 3.10 que depende da constante de acoplamento $\kappa=\beta L / V_{m}$. Portanto, o ganho calculado dessa forma é o ganho total fornecido pelo meio de comprimento $L$. No entanto, para determinar o comportamento dos campos médios no interior do meio de ganho, introduziremos a variável auxiliar $\xi=\beta d / V_{m}$, onde $d=t V_{m}$ é o parâmetro que descreve a distância percorrida no interior do meio de ganho, dessa forma, podemos substituir $\kappa$ por $\xi$ na equação 3.10 e calcular o ganho do campo de prova enquanto se propaga no meio $\chi^{3}$. Sabendo que as potências dos campos de bombeio e de prova na entrada do meio de ganho são dadas por $P_{0}(0)$ e $P_{1}(0)$, e que a distância $d$ é proporcional à $\xi$ as potências dos campos de bombeio e prova ao longo do meio de ganho são dadas por

$$
\begin{aligned}
& P_{0}(\xi)=P_{0}(0)-2 G(\xi), \\
& P_{1}(\xi)=P_{1}(0)+G(\xi),
\end{aligned}
$$

respectivamente. Assim, as amplitudes dos campos de bombeio e prova podem ser determinadas por meio da relação $\alpha_{n}(\xi)=\sqrt{P_{n}(\xi)}$.

O leitor deve observar que os campos $\alpha_{n}(\xi)$ não estão completamente determinados, afinal não conhecemos o comportamento de $P_{n}(0)$. No entanto, para determinar esse comportamento é necessário fazer considerações sobre a realimentação provocada pela cavidade. Com essa informação encontraremos a curva que expressa a potência de saída em função da potência de entrada e em função da reflectância. Nas seções que se seguem estudaremos os campos médios de dois tipos de cavidades diferentes, as cavidades duplamente e triplamente ressonantes.

\subsection{Campo médio do OPO duplamente ressonante}

Nessa seção estudamos o sistema em que os feixes de prova e conjugado cavitam e o feixe de bombeio faz uma passagem simples pelo meio de ganho. A cavidade em anel vista na figura 3.2 é uma representação teórica do OPO duplamente ressonante, DROPO. A cavidade em anel é formada por dois espelhos de reflectâncias $R_{1}=R_{2}=1$ e um espelho de reflectância R. Os comprimentos de onda dos feixes de bombeio, prova e conjugado são muito próximos, sendo assim, eles são refletidos pelos espelhos da cavidade aproximadamente com a mesma reflectância. O campo de bombeio possui polarização vertical, enquanto os campos de prova e conjugado possuem polarização horizontal. Assim, os PBSs garantem que o bombeio fará uma passagem simples pelo meio de ganho mas não interferem no caminho dos campos de prova e conjugado. No interior dessa cavidade está a célula de rubídio. A propagação por esse meio é descrita em função da distância $d$ percorrida pelos feixes em seu interior, ou seja, as potências dos feixes de bombeio, prova e conjugado são dadas por $P_{0}(d), P_{1}(d)$ e $P_{2}(d)$. Na figura 3.2 vemos uma célula de rubídio de comprimento L com os feixes de bombeio, prova e conjugado entrando no meio de ganho pelo ponto $d=0$ com potências dadas por $P_{0}(0), P_{1}(0)$ e $P_{2}(0)$ e 
na saída da célula de rubídio, $d=L$, as potências dos feixes de bombeio, prova e conjugado são dadas por $P_{0}(L), P_{1}(L)$ e $P_{2}(L)$.

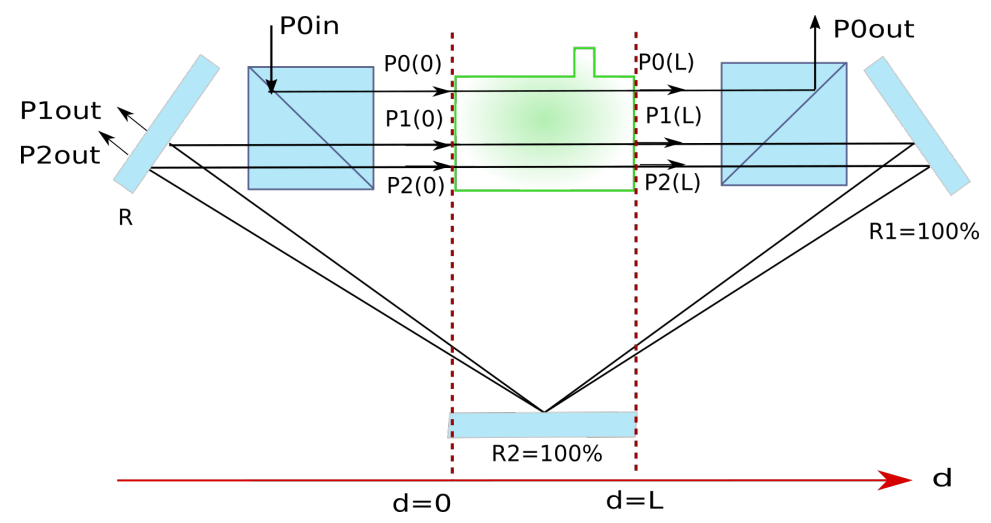

Figura 3.2: OPO duplamente ressonante: A cavidade consiste de dois espelhos $100 \%$ refletores, $R_{1}$ e $R_{2}$ e um espelho de reflectância $\mathrm{R}$. O campo de bombeio é possui polarização vertical, enquanto os campos de prova e conjugado possuem polarização horizontal. Assim, os PBS garantem que o bombeio fará uma passagem simples pelo meio de ganho mas não interferem no caminho dos campos de prova e conjugado. Para ilustrar a variação da potência dos feixes de bombeio, subíndice 0, prova, subíndice 1, e conjugado, subíndice 2, ao longo de seu percurso na cavidade destaca-se a potência em pontos de interesse. Em d=0 tem-se $P_{0}(0), P_{1}(0)$ e $P_{2}(0)$, em d=L, $P_{0}(d), P_{1}(d)$ e $P_{2}(d)$, saindo pelo espelho $R, P_{1 o u t}, P_{2 o u t}$, para o bombeio as potências de entrada e saída no experimento são dadas por $P_{0 i n}$ e $P_{0 o u t}$.

A potência do feixe de bombeio injetada na cavidade é dada por $P_{0 i n}$, como o bombeio faz uma passagem simples pela cavidade $P_{0 \text { in }}=P_{0}(0)$. Sendo $P_{0 o u t}$ a potência do feixe de bombeio que deixa a cavidade, $P_{\text {oout }}=P_{0}(L)$ por se tratar de uma passagem simples. Lembrando o resultado obtido pelas equações 3.8 , encontramos que potência de saída do bombeio, $P_{0 o u t}$, em função da potência de entrada, $P_{0 i n}$, é dada por:

$$
P_{0 o u t}=P_{0 i n}-2 G
$$

Lembrando que a potência da semente do feixe de prova é desprezível em comparação à potência feixe de bombeio e percebendo que o ganho e os efeitos devido à reflexão pelos espelhos da cavidade são os mesmos, concluímos que os feixes de prova e conjugado possuem a mesma potência em qualquer ponto do sistema. Assim, a partir desse momento serão apresentados apenas os resultados para o feixe de prova, podendo-se estender as conclusões ao feixe conjugado.

Se a cavidade está em equilíbrio, esperamos que em uma volta completa $P_{1}(0)$ seja o resultado da reinjeção da parcela refletida de $P_{1}(L)$ no meio de ganho. Reescrevendo $P_{1}(L)$ em termos de $P_{1}(0)$, obtemos $P_{1}(0)=R\left(P_{1}(0)+G\right)$. Rearranjando:

$$
P_{1}(0)=R G /(1-R)
$$


As potências dos feixes de prova e conjugado na saída do espelho de reflectância $R$ são dadas por $P_{1 \text { out }}$ e $P_{2 o u t}$. Pelas condições de contorno no espelho de reflectividade $\mathrm{R}$ encontramos:

$$
P_{\text {1out }}=\frac{(1-R)}{R} P_{1}(0)
$$

Das equações 3.15 e 3.16 concluímos que:

$$
P_{\text {lout }}=G
$$

O resultado da equação 3.17 já era esperado para uma cavidade em equilíbrio, pois nessas condições toda a potência que entra na cavidade, $P_{0 i n}$, deve deixar a cavidade. A parcela da potência de entrada não expulsa por $P_{0 \text { out }}$ é $2 G$, sendo $P_{1 \text { out }}=P_{2 \text { out }}$ concluímos que $P_{1 \text { out }}=G$.

O objetivo nesse momento é encontrar a potência de entrada na cavidade em função da potência de saída para o caso em estudo nessa seção (cavidade sem o feixe de bombeio). Substituindo a equação 3.15 em 3.10 obtemos:

$$
\frac{1-R}{R}=\frac{P_{0}(0)\left(e^{2 \kappa\left(P_{0}(0)+2 P_{1}(0)\right)}-1\right)}{2 P_{1}(0) e^{2 \kappa\left(P_{0}(0)+2 P_{1}(0)\right)}+P_{0}(0)}
$$

Devido à dificuldade de tratar a expressão acima utilizamos auxílio computacional para obter $P_{1}(0)$ em função da reflectância e de $P_{0}(0)$, o que equivale a obter a potência de saída $P_{1 o u t} \mathrm{em}$ função da reflectância e da potência de entrada $P_{0 i n}$. A partir desse resultado é feito o gráfico 3D apresentado na figura 3.3 que expressa a potência do feixe de prova no interior da cavidade em função da potência de entrada do feixe de bombeio e da refletividade para $\kappa=3$.

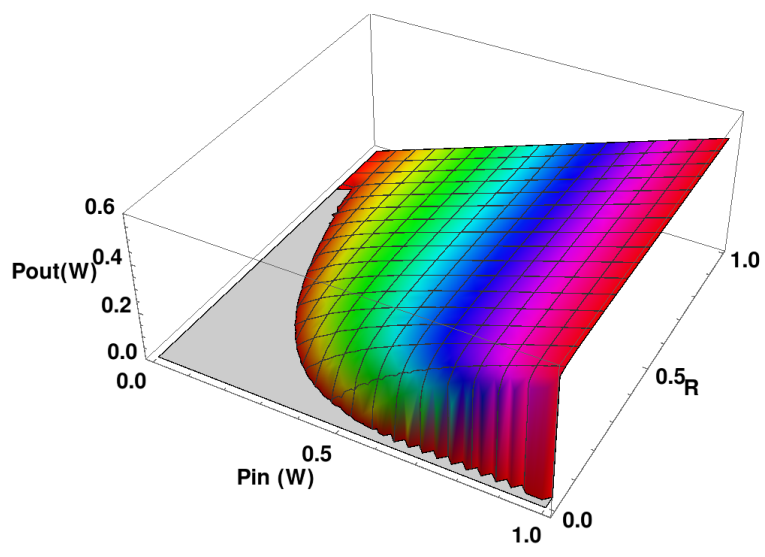

Figura 3.3: Potência do feixe de prova por reflectividade e por potência de entrada do bombeio.

Como estamos considerando o caso sem semente do feixe de prova, até que tenhamos ultrapassado a potência limiar do bombeio, $P_{t h}$, temos $P_{1}(0)=0$. Após ultrapassarmos esse limiar a potência cresce de forma contínua a partir de zero. Dessa forma, para uma potência 
$P_{0}(0)$ próxima de $P_{t h}$ mas um pouco maior $P_{1}(0) \ll P_{0}(0)$. Assim, a equação 3.18 pode ser simplificada para $\frac{1}{R}-1=e^{2 \kappa\left(P_{0}(0)\right)}-1$. Resolvendo essa expressão encontramos:

$$
P_{0 t h}=\frac{-\ln R}{2 \kappa}
$$

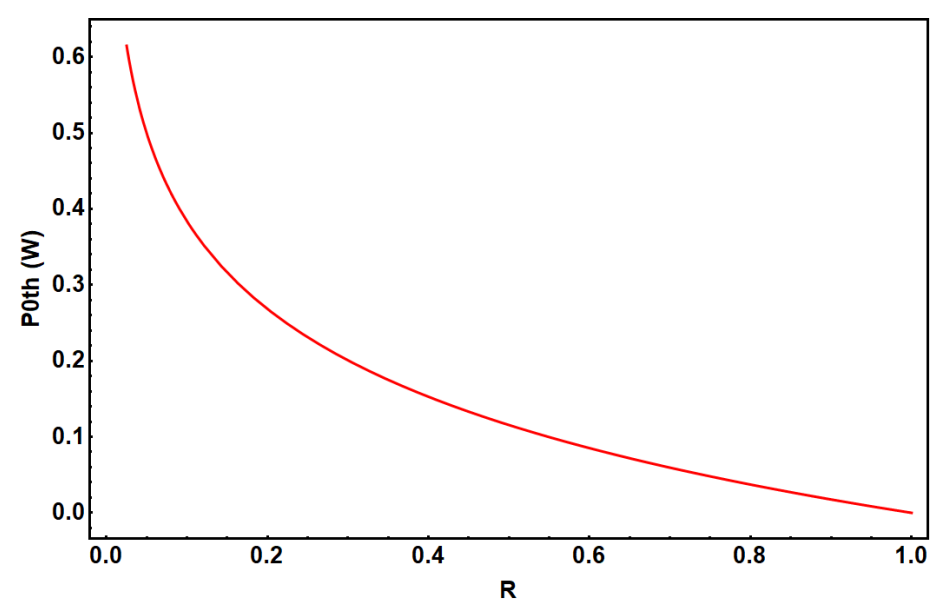

Figura 3.4: Potência limiar do bombeio, $P_{t h}$, em função da reflectância da cavidade $\mathrm{R}$ para um valor de $\kappa$ fixo em $3 \mathrm{~W}^{-1}$.

Na figura 3.4 fixamos $\kappa=3 \mathrm{~W}^{-1}$ e observamos o comportamento $P_{t h}$ em função de $R$. Podemos ver que a cavidade possui um limiar de oscilação que diminui com o aumento da reflectância da cavidade. Esse resultado já era esperado, pois mantendo-se a potência de bombeio fixa o aumento da reflectância da cavidade faz com que a potência intracavidade aumente. Assim, cavidades com reflectâncias maiores atingem mais rapidamente potência intracavidade necessária para amplificar os modos de prova e conjugado do vácuo até o limiar de oscilação.

(a)

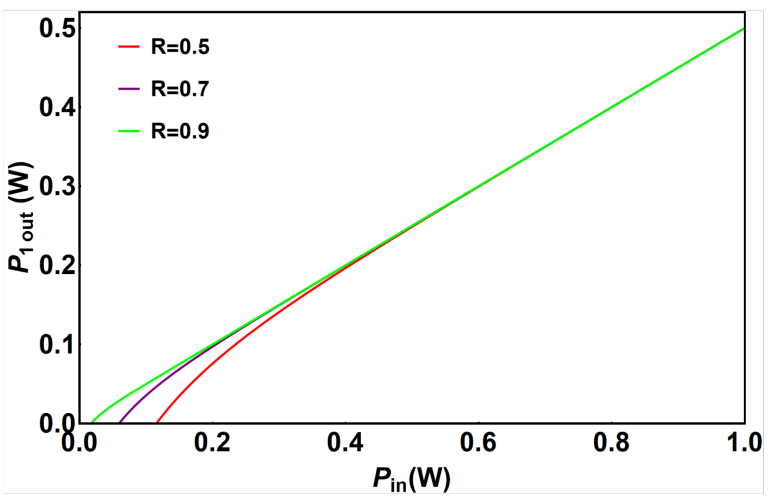

(b)

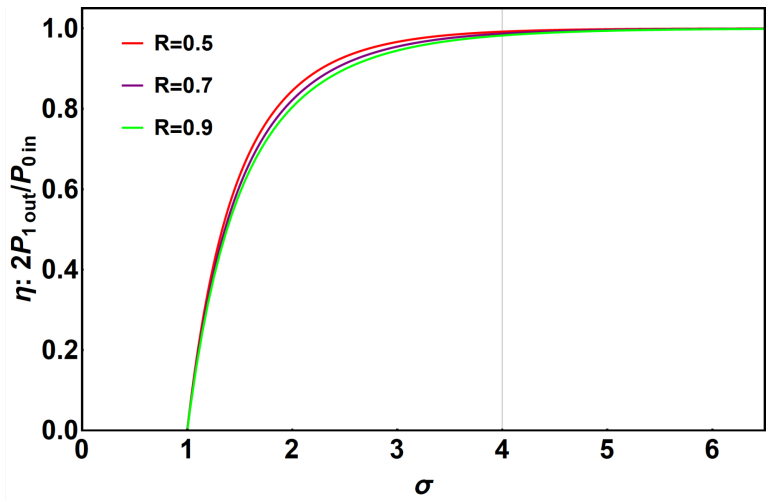

Figura 3.5: (a) Comportamento da potência de saída da cavidade do feixe de prova em função da potência de entrada do bombeio para diferentes reflectâncias da cavidade. (b) Eficiência da conversão do feixe de prova nos feixes prova e conjugado para diferentes reflectâncias da cavidade. 
A figura 3.5 (a) ilustra a potência de saída do feixe de prova $P_{1 o u t}$ em função da potência de bombeio $P_{0 i n}$. Nessa figura vemos que após a potência de bombeio ultrapassar o limiar de oscilação, a potência de saída aumenta com a potência de entrada e para regiões distantes do limiar $P_{1 \text { out }}$ cresce linearmente com $P_{0 \text { in }}$ obedecendo a relação: $P_{1 \text { out }}=P_{0 \text { in }} / 2$.

Uma forma equivalente de analisar esse processo é por meio da eficiência que é dada pela razão entre a potência de entrada do bombeio na cavidade e a soma das potências de saída dos feixes de prova e conjugado, $\eta=2 P_{1 \text { out }} / P_{0 i n}$. A análise da eficiência em função da potência do bombeio normalizada pela potência limiar, $\sigma=P_{0 i n} / P_{0 t h}$, é mostrada na figura 3.5 (b). Podemos ver que as curvas da eficiência de conversão são muito parecidas para todas as reflectâncias analisadas. Vemos que em todos os casos a eficiência evolui assintoticamente para 1, mas cavidades com menor reflectância evoluem a uma taxa ligeiramente maior.

Nessa seção concluímos o estudo clássico do OPO duplamente ressonante tendo sido determinadas as potências médias dos campos na saída do OPO, em função das potência de entrada do bombeio e da reflectância da cavidade. O estudo dos campos médios será importante para o cálculo quântico. Na próxima etapa desse trabalho, estudaremos o comportamento do OPO triplamente ressonante (TROPO) para comparação com o DROPO.

\subsection{Campo médio do OPO triplamente ressonante}

Uma configuração alternativa do trabalho que estamos fazendo é o OPO triplamente ressonante, TROPO, nessa configuração os campos de bombeio, prova e conjugado cavitam de acordo com o esquema apresentado na figura 3.6. Nessa figura vemos uma cavidade em anel formada por dois espelhos de reflectâncias $R_{1}=R_{2}=1$ e um espelho de reflectância $R$. No interior dessa cavidade está a célula de rubídio. A propagação por esse meio é descrita em função da distância $d$ percorrida pelos feixes em seu interior, ou seja, as potências dos feixes de bombeio, prova e conjugado são dadas por $P_{0}(d), P_{1}(d)$ e $P_{2}(d)$.

Iniciamos a análise do TROPO apontando suas semelhanças com o DROPO. Devemos notar que as equações que descrevem a propagação do campo de prova dentro da cavidade no caso do TROPO são as mesmas que descrevem o DROPO, sendo assim, as equações 3.15, 3.16 e 3.17 continuam válidas no caso triplamente ressonante. Além disso, o ganho do campo de prova, obtido pela expressão 3.10 depende dos campos $P_{0}(0), P_{1}(0)$ e da constante $\kappa$, portanto o ganho continua o mesmo no caso do TROPO. Lembrando que chegamos à relação 3.18 unindo as informações de 3.15 e 3.10, esta também continua válida.

A primeira diferença entre os sistemas TROPO e DROPO que devemos notar é que quando tratamos o problema dos campos médios para o DROPO a potência de entrada do bombeio era igual à potência intracavidade no ponto zero, $P_{0 \text { in }}=P_{0}(0)$. Essa igualdade simplifica bastante o problema, pois nesse caso, as equações 3.16 e 3.10 são o suficiente para relacionarmos a potência de saída do campo de prova à potência de entrada do bombeio. No caso do TROPO, a união 


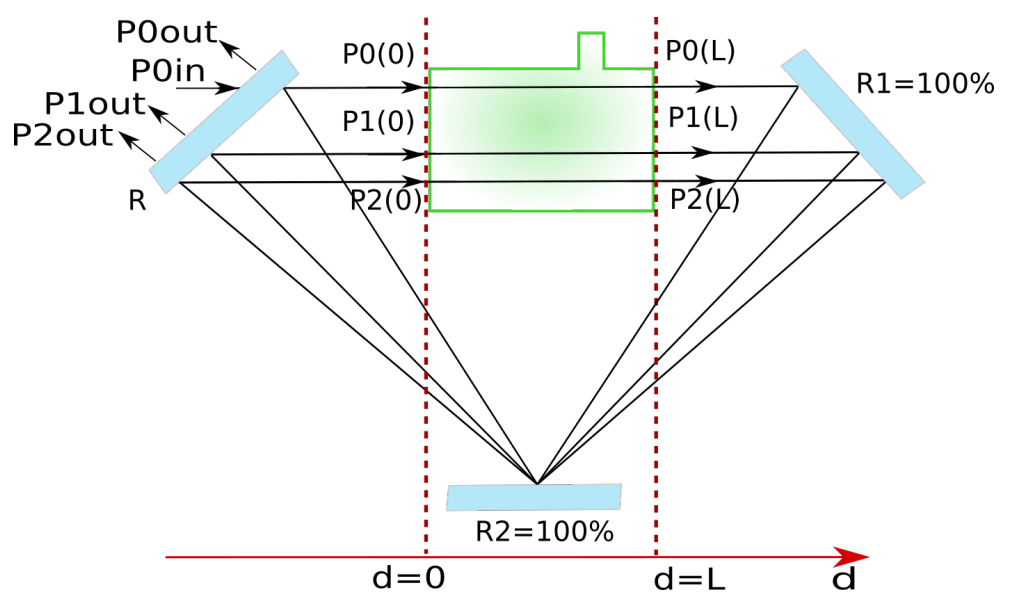

Figura 3.6: TROPO: meio de ganho $\chi^{3}$ no interior de uma cavidade em anel triplamente ressonante. A cavidade consiste de dois espelhos $100 \%$ refletores, $R_{1}$ e $R_{2}$ e um espelho de reflectância R. Para ilustrar a variação da potência dos feixes de bombeio, subíndice 0, prova, subíndice 1, e conjugado, subíndice 2, ao longo de seu percurso na cavidade destaca-se a potência em pontos de interesse. Em d=0 tem-se $P_{0}(0), P_{1}(0)$ e $P_{2}(0)$, em $\mathrm{d}=\mathrm{L}, P_{0}(d), P_{1}(d)$ e $P_{2}(d)$, saindo pelo espelho $R, P_{1 o u t}, P_{2 o u t}$, para o bombeio as potências de entrada e saída no experimento são dadas por $P_{0 \text { in }}$ e $P_{0 o u t}$.

dessas informações fornece a potência de saída do campo de prova em função da potência do campo de bombeio no ponto zero. Esse não é exatamente o resultado que nos interessa, então vamos estudar um pouco melhor a propagação do campo de bombeio no interior da cavidade.

Para simplificar a equação que descreve o ganho do campo de prova e as equações que descrevem a propagação dos campos de bombeio e prova precisamos notar que a evolução dos campos de bombeio, prova e conjugado acontece de forma interligada. Sabemos que em uma situação de equilíbrio a potência total intracavidade, $P_{T 0}$, é a mesma em qualquer ponto em seu interior, o que nos fornece a relação

$$
P_{T 0}=P_{0}(d)+2 P_{1}(d)=P_{0}(0)+2 P_{1}(0)=P_{0}(L)+2 P_{1}(L) .
$$

Dito isso, notamos que o expoente das duas exponenciais na equação 3.18 é dado por $P_{0}(0)+$ $2 P_{1}(0)$. Então, podemos reescrever a equação 3.18 como

$$
\frac{P_{1}(0)}{P_{0}(0)}=\frac{R e^{2 \kappa P_{T 0}}-1}{2(1-R) e^{2 \kappa P_{T 0}}} .
$$

De acordo com a equação 3.21, a razão $P_{1}(0) / P_{0}(0)$ só depende de $P_{T 0}$, o que nos inspira a tentar descrever $P_{0}(0)$ e $P_{1}(0)$ separadamente, mas em função de $P_{T 0}$ e $P_{i n}$. Além disso, é interessante observar que só utilizamos relações válidas tanto para o DROPO quanto para o TROPO para chegar a equação 3.21 e que, portanto, essa equação é válida para os dois casos.

O próximo passo do nosso estudo é utilizar as equações de propagação do bombeio para 
executar a tarefa de reescrever $P_{0}(0)$ e $P_{1}(0)$ em função de $P_{T 0}$ e $P_{i n}$. Então, para o campo de bombeio cavitando, as equações de autoconsistência são:

$$
\begin{aligned}
& \sqrt{P_{0}(0)}=t \sqrt{P_{0 i n}}-r \sqrt{P_{0}(L)} \\
& \sqrt{P_{0 o u t}}=t \sqrt{P_{0}(L)}+r \sqrt{P_{0 i n}} .
\end{aligned}
$$

Rearranjamos os termos da equação 3.22 para obter $\sqrt{P_{0}(d)}$ em função dos demais campos:

$$
\sqrt{P_{0}(L)}=\frac{t \sqrt{P_{0 i n}}-\sqrt{P_{0}(0)}}{r}
$$

Substituindo a equação 3.24 na equação 3.23 conseguimos chegar a uma expressão:

$$
P_{0 o u t}=\frac{P_{0 i n}-2 t \sqrt{P_{0 i n} P_{0}(0)}}{R}
$$

e, sabendo que conservação da energia nos fornece uma relação entre os campos de entrada e saída da cavidade:

$$
P_{0 \text { in }}=P_{0 o u t}+2 P_{1 o u t},
$$

rearranjamos a equação 3.26 na forma $P_{0 o u t}=P_{\text {in }}-2(1-R) P_{1}(0) / R$ e substituímos em 3.25 . Reagrupando alguns termos dessa nova expressão para formar $P_{T 0}$ de acordo com a equação 3.20, chegamos à equação

$$
P_{0}(0)=\frac{1-R}{4} \frac{\left(P_{0 i n}+P_{T 0}\right)^{2}}{P_{0 i n}},
$$

que descreve $P_{0}(0)$ em termos de $P_{T 0}$ e $P_{0 i n}$.

Lembrando que $P_{0}(L)$ é resultado da propagação do campo de bombeio pelo meio de ganho temos que $P_{0}(L)=P_{0}(L)-2 G=P_{0}(0)-2(1-R) P_{1}(0) / R$. Substituímos essa relação na equação 3.22 e reagrupamos alguns de seus termos de acordo com 3.20. Dessa forma, chegamos à relação

$$
P_{1}(0)=\frac{R-1}{8} \frac{\left(P_{0 i n}-P_{T 0}\right)^{2}}{P_{0 i n}}+\frac{R P_{T 0}}{2},
$$

que descreve $P_{1}(0)$ em termos de $P_{T 0}$ e $P_{0 i n}$.

As equações 3.27 e 3.28 descrevem o comportamento de $P_{0}(0)$ e $P_{1}(0)$ em função de $P_{T 0}(0)$ e $P_{0 i n}$. Então, substituímos essas equações em $P_{0}(0)$ e $P_{1}(0)$, respectivamente. Dessa forma, chegamos a uma expressão que relaciona a potência total intracavidade à potência de entrada do bombeio

$$
-(1-R)\left(P_{0 i n}-P_{T 0}\right)^{2}+4 R P_{0 i n} P_{T 0}=\left(R-e^{2 \kappa P_{T 0}}\right)\left(P_{0 i n}+P_{T 0}\right)^{2},
$$

a qual utilizamos para encontrar uma solução numérica que fornece $P_{T 0}$ em função de $P_{i n}$. 
Substituímos a solução numérica para $P_{T 0}$ nas equações 3.27 e 3.28, dessa forma, obtivemos $P_{0}(0)$ e $P_{1}(0)$ em função de $P_{i n}$. A potência de saída do campo de bombeio é dada pela equação 3.25, substituímos a solução numérica para $P_{0}(0)$ nessa equação e obtivemos o campo de saída do bombeio em função do campo de entrada. De forma semelhante, obtivemos a potência de saída do campo de prova substituindo a solução numérica de $P_{1}(0)$ na equação 3.16.

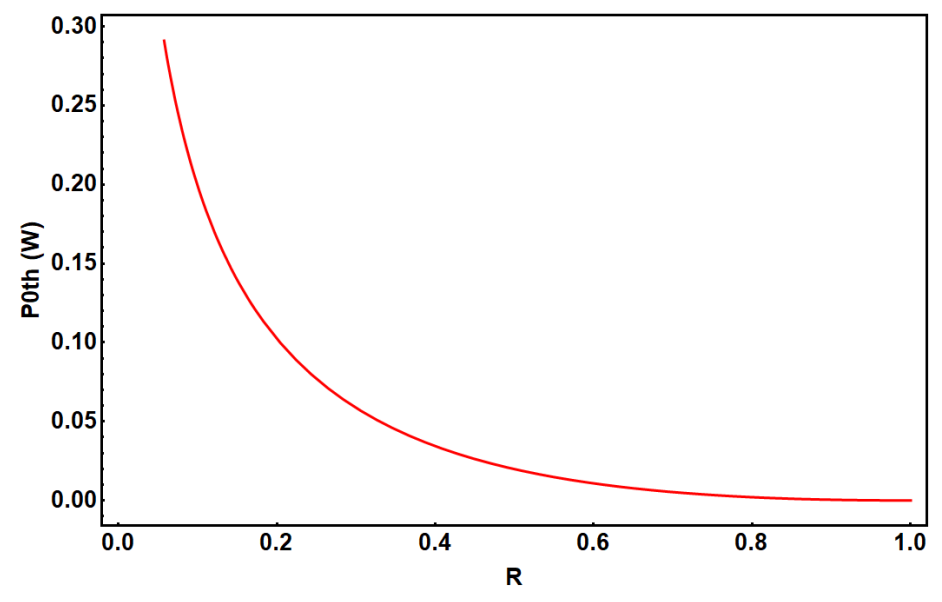

Figura 3.7: Potência limiar do bombeio, $P_{\text {inth }}$, em função da reflectância da cavidade $\mathrm{R}$ para um valor de $\kappa$ fixo em $3 \mathrm{~W}^{-1}$.

Quando estudamos o DROPO na seção 3.3 determinamos a potência limiar do bombeio, estabelecendo que $P_{1}(0) \ll P_{0}(0)$ na equação 3.18. Esse raciocínio continua válido no caso do TROPO, porém, encontrar $P_{0 t h}$ significa encontrar a potência do bombeio intracavidade quando atingimos o limiar de oscilação. Para encontrarmos a potência de entrada do bombeio no caso do TROPO, observamos que para esse sistema $P_{0 t h}=P_{T 0}$. Lembrando que a equação 3.29 relaciona $P_{0 t h}$ e $P_{0 i n}$, substituímos a equação 3.18 em 3.29 e assim encontramos uma expressão analítica para a potência limiar do bombeio

$$
P_{\text {inth }}=\frac{(\sqrt{R}-1) \ln (R)}{3 \kappa(\sqrt{R}+1)},
$$

cujo comportamento é apresentado na figura 3.7. Observamos que o comportamento de $P_{\text {inth }}$ é muito parecido com o comportamento de $P$ th, pois em ambos os casos temos a potência limiar decrescendo de acordo com uma função proporcional a $-\ln R$. O decrescimento de Pth com o aumento de $\mathrm{R}$ já era esperado, visto que com o aumento de $\mathrm{R}$ aumentamos a potência intracavidade.

A figura 3.8 (a) apresenta o gráfico da potência de saída do campo de prova em função da potência de entrada do bombeio para diferentes reflectâncias $(R=0,5,0,7$ e 0,9). Podemos ver que o comportamento do TROPO é muito diferente do comportamento do DROPO (figura 3.5) que apresentava um comportamento praticamente linear para potências de bombeio muito 
maiores que a potência limiar. No caso do TROPO, mesmo para regiões distantes do limiar, o crescimento não é linear e se torna menos expressivo à medida que a potência do bombeio cresce. Além disso observamos que cavidades com maior reflectância convertem uma menor fração da potência de bombeio nos campos de prova e conjugado.

A figura 3.8 (b) apresenta a eficiência da conversão $2 P_{1 \text { out }} / P_{0 i n}$ em função da potência de bombeio normalizada pela potência limiar para diferentes reflectâncias da cavidade. Vemos que o comportamento das três curvas apresentadas (reflectância igual a 0,5, 0,7 e 0,9) é muito parecido nas proximidades do limiar de oscilação. As diferenças entre as curvas só se tornam apreciáveis após $\sigma=20$, quando observamos uma queda na eficiência de conversão mais apreciável para cavidades com reflectâncias maiores. Além disso, vemos que a maior eficiência de conversão ocorre aproximadamente em $\sigma=4$ quando temos eficiência de aproximadamente $100 \%$, para $\sigma>4$ essa eficiência cai bastante, para $\sigma=50$ todas as curvas apresentam eficiência em torno de $50 \%$. Esse resultado também foi observado em outros trabalhos com o OPO $\chi^{2}$ [52]. Devemos observar que esse caso é muito distinto do comportamento do DROPO cuja eficiência crescia assintoticamente para 1 em grandes potências de bombeio.

(a)

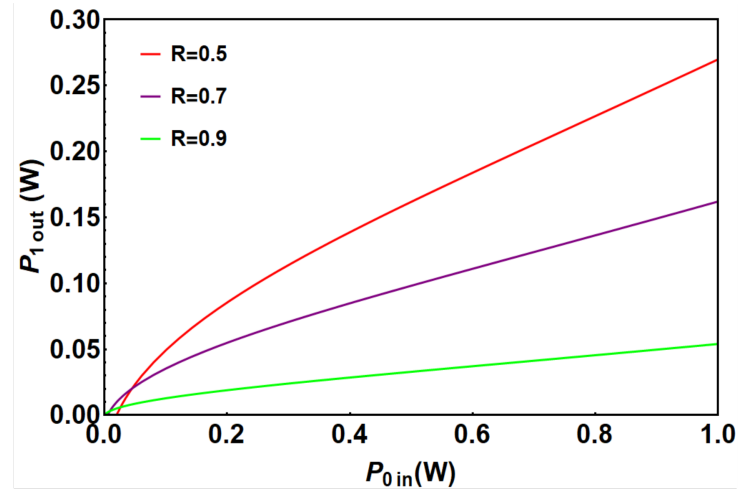

(b)

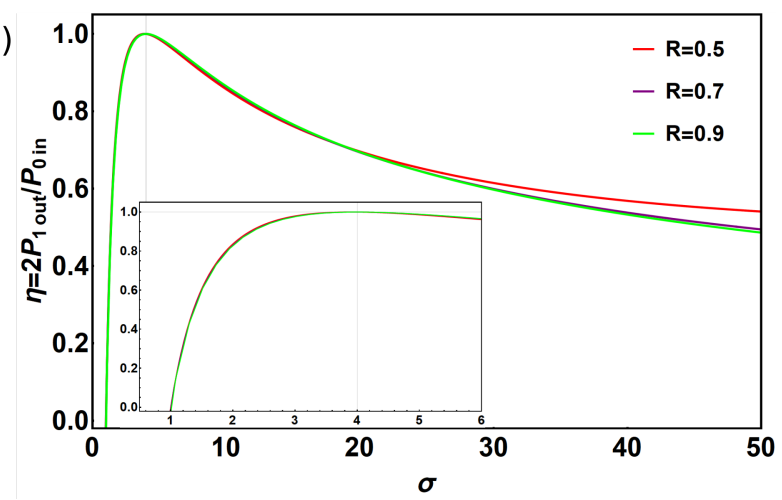

Figura 3.8: (a) Comportamento da potência de saída da cavidade do feixe de prova por potência de entrada do bombeio para diferentes reflectâncias da cavidade. (b) Eficiência da conversão do feixe de prova nos feixes prova e conjugado para diferentes reflectâncias da cavidade.

\subsection{Ganho do 4WM em termos das bandas laterais}

O objetivo dessa seção é fazer uma análise das flutuações do campo quantizado no domínio da frequência para o OPO $\chi^{3}$. Faremos isso com base no tratamento apresentado no trabalho [53]. Esse estudo envolve a análise dos campos elétricos do bombeio, prova e conjugado cujas portadoras possuem frequências $\omega_{0}, \omega_{1}$ e $\omega_{2}$, respectivamente. Por esse motivo é necessário que a notação do operador de aniquilação deixe claro a qual campo estamos nos referindo. Assim, os operadores $\hat{a}(t)$ e $\hat{a}_{\omega}$ ganham um subíndice $n$ para incluir a informação do campo a que se referem, ou seja, $\hat{a}(t) \rightarrow \hat{a}_{n}(t)$ e $\hat{a}_{\omega} \rightarrow \hat{a}_{n \omega}$. Seguiremos considerando que $n=0,1,2$ se referem 
aos modos do bombeio, prova e conjugado, respectivamente. Dessa forma, a equação 2.16 pode ser reescrita como:

$$
\hat{a}_{n}(t)=\alpha_{n}+\int^{\prime} d \Omega \hat{a}_{n \Omega} e^{-i \Omega t}
$$

em que $\alpha_{n}$ representa o valor médio da amplitude da portadora, $\alpha_{n}=\left\langle\hat{a}_{n(\Omega=0)}\right\rangle$. Expandindo o Hamiltoniano de interação do 4WM

$$
\hat{H}_{\chi}=i \hbar \beta \hat{a}_{0}^{2} \hat{a}_{1}^{\dagger} \hat{a}_{2}^{\dagger}+\text { h.c. }
$$

em termos da equação 3.31. O produto dos operadores $\hat{a}_{0}, \hat{a}_{1}, \hat{a}_{2}$ é reescrito como:

$$
\hat{a}_{0}^{2} \hat{a}_{1}^{\dagger} \hat{a}_{2}^{\dagger}=\left(\alpha_{0}+\int^{\prime} d \Omega \hat{a}_{0 \Omega} e^{-i \Omega t}\right)^{2}\left(\alpha_{1}^{*}+\int^{\prime} d \Omega \hat{a}_{1 \Omega}^{\dagger} e^{i \Omega t}\right)\left(\alpha_{2}^{*}+\int^{\prime} d \Omega \hat{a}_{2 \Omega}^{\dagger} e^{i \Omega t}\right)
$$

Após eliminar os termos referentes somente aos valores médios, os termos que não satisfazem a condição de casamento de fase e termos que dependem das bandas laterais em terceira ordem ou ordens superiores, o produto dos operadores é dado por:

$$
\begin{aligned}
\hat{a}_{0}^{2} \hat{a}_{1}^{\dagger} \hat{a}_{2}^{\dagger}= & \alpha_{0}^{2} \int^{\prime} d \Omega d \Omega^{\prime} \hat{a}_{1 \Omega}^{\dagger} \hat{a}_{2 \Omega^{\prime}}^{\dagger} e^{i\left(\Omega+\Omega^{\prime}\right) t}+2 \alpha_{0} \alpha_{1}^{*} \int^{\prime} d \Omega d \Omega^{\prime} \hat{a}_{0 \Omega} \hat{a}_{2 \Omega^{\prime}}^{\dagger} e^{i\left(\Omega-\Omega^{\prime}\right) t} \\
& +2 \alpha_{0} \alpha_{2}^{*} \int^{\prime} d \Omega d \Omega^{\prime} \hat{a}_{0 \Omega} \hat{a}_{1 \Omega^{\prime}}^{\dagger} e^{i\left(\Omega-\Omega^{\prime}\right) t}+\alpha_{1}^{*} \alpha_{2}^{*} \int^{\prime} d \Omega d \Omega^{\prime} \hat{a}_{0 \Omega} \hat{a}_{0 \Omega^{\prime}} e^{i\left(\Omega+\Omega^{\prime}\right) t} .
\end{aligned}
$$

Para que haja casamento de fase para cada um dos termos restantes, é necessário que $\Omega^{\prime}=-\Omega$ para o primeiro e para o último termo, e que $\Omega^{\prime}=\Omega$ para os demais termos. Assim, esse produto se torna:

$$
\hat{a}_{0}^{2} \hat{a}_{1}^{\dagger} \hat{a}_{2}^{\dagger}=\int^{\prime} d \Omega\left[\alpha_{0}^{2} \hat{a}_{1 \Omega}^{\dagger} \hat{a}_{2(-\Omega)}^{\dagger}+2 \alpha_{0} \alpha_{1}^{*} \hat{a}_{0 \Omega} \hat{a}_{2 \Omega}^{\dagger}+2 \alpha_{0} \alpha_{2}^{*} \hat{a}_{0 \Omega} \hat{a}_{1 \Omega}^{\dagger}+\alpha_{1}^{*} \alpha_{2}^{*} \hat{a}_{0 \Omega} \hat{a}_{0(-\Omega)}\right]
$$

Logo, o Hamiltoniano no espaço das frequências é dado por:

$$
\begin{aligned}
H_{\chi \Omega}= & i \hbar \beta\left(\left(\alpha_{0}^{2} \hat{a}_{1 \Omega}^{\dagger} \hat{a}_{2(-\Omega)}^{\dagger}+\alpha_{0}^{2} \hat{a}_{1(-\Omega)}^{\dagger} \hat{a}_{2 \Omega}^{\dagger}\right)+\left(2 \alpha_{0} \alpha_{1}^{*} \hat{a}_{0 \Omega} \hat{a}_{2 \Omega}^{\dagger}+2 \alpha_{0} \alpha_{1}^{*} \hat{a}_{0(-\Omega)} \hat{a}_{2(-\Omega)}^{\dagger}\right)\right. \\
& +\left(2 \alpha_{0} \alpha_{2}^{*} \hat{a}_{0 \Omega} \hat{a}_{1 \Omega}^{\dagger}+2 \alpha_{0} \alpha_{2}^{*} \hat{a}_{0(-\Omega)} \hat{a}_{1(-\Omega)}^{\dagger}\right)+\left(\alpha_{1}^{*} \alpha_{2}^{*} \hat{a}_{0 \Omega} \hat{a}_{0(-\Omega)}+\left(\alpha_{1}^{*} \alpha_{2}^{*} \hat{a}_{0(-\Omega)} \hat{a}_{0 \Omega}\right)\right)+\text { h.c.. }
\end{aligned}
$$

Dessa forma, o Hamiltoniano no espaço do tempo se relaciona ao Hamiltoniano no espaço das frequências (após eliminarmos o valor médio, termos sem casamento de fase e termos de ordem superior a dois nas bandas laterais) por:

$$
H_{\chi}=\int_{\epsilon}^{\infty} d \Omega H_{\chi \Omega}
$$


Onde $\epsilon>0$ é a frequência de corte das bandas laterais. Esse Hamiltoniano possui termos em que as bandas superiores e inferiores estão acopladas. Sendo assim, é conveniente fazer uma mudança para a base dos operadores simétrico e antissimétrico:

$$
\begin{aligned}
& \hat{a}_{n s}=\frac{1}{\sqrt{2}}\left[\hat{a}_{n \Omega}+\hat{a}_{n(-\Omega)}\right] \\
& \hat{a}_{n a}=\frac{1}{\sqrt{2}}\left[\hat{a}_{n \Omega}-\hat{a}_{n(-\Omega)}\right]
\end{aligned}
$$

Os operadores $\hat{a}_{n \Omega}$ e $\hat{a}_{n(-\Omega)}$ podem ser reescritos nessa nova base como:

$$
\begin{aligned}
\hat{a}_{n \Omega} & =\frac{1}{\sqrt{2}}\left[\hat{a}_{n s}+\hat{a}_{n a}\right], \\
\hat{a}_{n(-\Omega)} & =\frac{1}{\sqrt{2}}\left[\hat{a}_{n s}-\hat{a}_{n a}\right] .
\end{aligned}
$$

Na nova base o Hamiltoniano pode ser separado em uma parte simétrica, $H_{\chi \Omega_{s}}$, e outra antissimétrica, $H_{\chi \Omega_{a}}$ :

$$
H_{\chi \Omega}=H_{\chi \Omega_{s}}+H_{\chi \Omega_{a}}
$$

que são dadas por:

$$
\begin{aligned}
& H_{\chi \Omega_{s}}=i \hbar \beta\left(\alpha_{0}^{2} \hat{a}_{1 s}^{\dagger} \hat{a}_{2 s}^{\dagger}+2 \alpha_{0} \alpha_{1}^{*} \hat{a}_{0 s} \hat{a}_{2 s}^{\dagger}+2 \alpha_{0} \alpha_{2}^{*} \hat{a}_{0 s} \hat{a}_{1 s}^{\dagger}+\alpha_{1}^{*} \alpha_{2}^{*} \hat{a}_{0 s}^{2}\right)+\text { h.c. } \\
& H_{\chi \Omega_{a}}=i \hbar \beta\left(-\alpha_{0}^{2} \hat{a}_{1 a}^{\dagger} \hat{a}_{2 a}^{\dagger}+2 \alpha_{0} \alpha_{1}^{*} \hat{a}_{0 a} \hat{a}_{2 a}^{\dagger}+2 \alpha_{0} \alpha_{2}^{*} \hat{a}_{0 a} \hat{a}_{1 a}^{\dagger}-\alpha_{1}^{*} \alpha_{2}^{*} \hat{a}_{0 a}^{2}\right)+\text { h.c. }
\end{aligned}
$$

A dinâmica do sistema é calculada na base dos operadores simétrico e antissimétrico:

$$
\frac{d \hat{a}_{n(s / a)}(t)}{d t}=\frac{1}{i \hbar}\left[H_{\chi(s / a)}, \hat{a}_{n(s / a)}^{\dagger}(t)\right]
$$

Lembrando que na seção 3.3 introduzimos a variável $d=t V_{m}$, concluímos que $\xi=\beta t$. Tanto os operadores $\hat{a}_{i}$ quanto as amplitudes $\alpha_{i}$ são dependentes de $\xi$, no entanto essa dependência será omitida por uma questão de simplicidade. Substituindo $t=\xi / \beta$ nas equações de Heisenberg, encontramos:

$$
\begin{aligned}
\frac{d}{d \xi} \hat{a}_{0(s / a)} & =-2 \alpha_{0}^{2} \alpha_{1} \hat{a}_{2(s / a)}^{\dagger}-2 \alpha_{0}^{*} \alpha_{2} \hat{a}_{1(s / a)}^{\dagger} \mp 2 \alpha_{1} \alpha_{2} \hat{a}_{0(s / a)}^{\dagger}, \\
\frac{d}{d \xi} \hat{a}_{1(s / a)} & = \pm \alpha_{0}^{2} \hat{a}_{2(s / a)}^{\dagger}+2 \alpha_{0} \alpha_{2}^{*} \hat{a}_{0(s / a)}, \\
\frac{d}{d \xi} \hat{a}_{2(s / a)} & = \pm \alpha_{0}^{2} \hat{a}_{1(s / a)}^{\dagger}+2 \alpha_{0}^{*} \alpha_{1} \hat{a}_{0(s / a)} .
\end{aligned}
$$


O conjunto de equações 3.42 pode ser reescrito de forma matricial por meio da introdução de um vetor $\overrightarrow{\mathbf{A}}_{s / a}$ dado por:

$$
\begin{aligned}
\overrightarrow{\mathbf{A}}_{s} & =\left(\begin{array}{llllll}
\hat{a}_{0(s)} & \hat{a}_{0(s)}^{\dagger} & \hat{a}_{1(s)} & \hat{a}_{1(s)}^{\dagger} & \hat{a}_{2(s)} & \hat{a}_{2(s)}^{\dagger}
\end{array}\right)^{T}, \\
\overrightarrow{\mathbf{A}}_{a} & =\left(\begin{array}{llllll}
\hat{a}_{0(a)} & \hat{a}_{0(a)}^{\dagger} & \hat{a}_{1(a)} & \hat{a}_{1(a)}^{\dagger} & \hat{a}_{2(a)} & \hat{a}_{2(a)}^{\dagger}
\end{array}\right)^{T}, \\
\overrightarrow{\mathbf{A}}_{s / a} & =\overrightarrow{\mathbf{A}}_{s} \otimes \overrightarrow{\mathbf{A}}_{a}
\end{aligned}
$$

e definindo a matriz $\mathbf{M}_{(s / a)}$ por:

$$
\mathbf{M}_{s / a}(\xi)=\left(\begin{array}{cccccc}
0 & \mp 2 \alpha_{1} \alpha_{2} & -2 \alpha_{0}^{*} \alpha_{2} & 0 & -2 \alpha_{0}^{*} \alpha_{1} & 0 \\
\mp 2 \alpha_{1}^{*} \alpha_{2}^{*} & 0 & 0 & 2 \alpha_{0} \alpha_{2}^{*} & 0 & 2 \alpha_{0} \alpha_{1}^{*} \\
2 \alpha_{0} \alpha_{2}^{*} & 0 & 0 & 0 & 0 & \pm \alpha_{0}^{2} \\
0 & 2 \alpha_{0}^{*} \alpha_{2} & 0 & 0 & \pm \alpha_{0}^{* 2} & 0 \\
2 \alpha_{0} \alpha_{1}^{*} & 0 & 0 & \pm \alpha_{0}^{2} & 0 & 0 \\
0 & 2 \alpha_{0}^{*} \alpha_{1} & \pm \alpha_{0}^{* 2} & 0 & 0 & 0
\end{array}\right)
$$

As equações 3.42 tomam a forma matricial:

$$
d \overrightarrow{\mathbf{A}}_{(s / a)} \overrightarrow{\mathbf{A}}_{(s / a)}^{-1}=\mathbf{M}_{(s / a)}(\xi) d \xi
$$

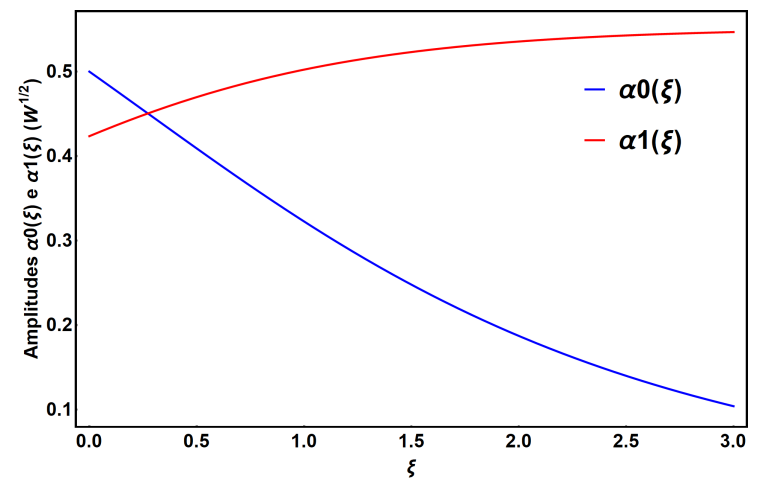

Figura 3.9: Amplitudes médias dos campos de bombeio, $\alpha_{0}(\xi)$, e prova, $\alpha_{1}(\xi)$, em função de $\xi$ para o caso do DROPO considerando a potência de entrada do bombeio de $P_{0 i n}(0)=0,25 \mathrm{~W}$ e $R=0,6$.

Analisando a equação 3.45 vemos que o vetor $\overrightarrow{\mathbf{A}}_{(s / a)}$ que contém os campos dos feixes de bombeio, prova e conjugado varia ao longo do meio de ganho como indica o gráfico 3.9 que contém os valores médios das amplitudes dos campos de bombeio e prova, sendo a amplitude do campo de conjugado igual a do prova. Resolvendo essa equação diferencial, esperamos encontrar 
uma solução da forma:

$$
\left.\overrightarrow{\mathbf{A}}_{(s / a)}\right|_{\xi=\kappa}=\left.\mathbf{G}_{(s / a)} \overrightarrow{\mathbf{A}}_{(s / a)}\right|_{\xi=0} .
$$

Como pode ser visto pelas equações 3.42, a taxa de conversão do bombeio nos feixes de prova e conjugado depende da potência dos feixes de bombeio, prova e conjugado ao longo do meio de ganho. Assim sendo, a taxa de conversão não é constante pois a depleção do bombeio é muito rápida, ou seja, sua potência diminui rapidamente durante sua passagem pelo meio de ganho. Portanto a matriz $\mathbf{M}_{(s / a)}$ é função de $\kappa$ e integrando a equação 3.45 obtemos:

$$
\mathbf{G}_{(s / a)}(\kappa)=\exp \left(\int_{0}^{\kappa} d \xi \mathbf{M}_{(s / a)}(\xi)\right)
$$

Com o auxílio computacional obtemos uma solução numérica para a equação 3.47. A figura 3.9 ilustra a depleção do campo médio do bombeio $\alpha_{0}$ e amplificação do campo de prova $\alpha_{1}$ para o caso de uma potência de bombeio de $P_{0}(0)=0,25 W$ e $R=0,6$ em função do parâmetro $\xi$ que é proporcional à distância percorrida pelos campos no interior da cavidade o que mostra a necessidade da integração feita na equação 3.47.

A matriz $\Lambda$ definida por:

$$
\Lambda=\frac{1}{\sqrt{2}}\left(\begin{array}{cc}
\mathbf{I}_{6} & \mathbf{I}_{6} \\
\mathbf{I}_{6} & -\mathbf{I}_{6}
\end{array}\right),
$$

em que $\mathbf{I}_{6}$ representa uma matriz identidade de ordem 6 . Tal matriz leva vetores do espaço das bandas simétrica e antissimétrica para o espaço das bandas laterais e vice-versa. Como por exemplo:

$$
\begin{aligned}
\overrightarrow{\mathbf{A}}_{( \pm \Omega)} & =\Lambda \overrightarrow{\mathbf{A}}_{(s / a)}, \\
\overrightarrow{\mathbf{A}}_{(s / a)} & =\Lambda \overrightarrow{\mathbf{A}}_{( \pm \Omega)},
\end{aligned}
$$

onde $\overrightarrow{\mathbf{A}}_{( \pm \Omega)}$ é a forma geral de um vetor que contém os modos dos campos de bombeio, prova e conjugado no espaço das bandas laterais:

$$
\begin{aligned}
& \overrightarrow{\mathbf{A}}_{(+\Omega)}=\left(\begin{array}{llllll}
\hat{a}_{0 \Omega} & \hat{a}_{0 \Omega}^{\dagger} & \hat{a}_{1 \Omega} & \hat{a}_{1 \Omega}^{\dagger} & \hat{a}_{2 \Omega} & \hat{a}_{2 \Omega}^{\dagger}
\end{array}\right)^{T},
\end{aligned}
$$

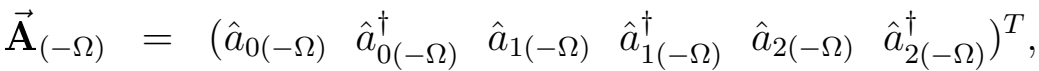

$$
\begin{aligned}
& \overrightarrow{\mathbf{A}}_{( \pm \Omega)}=\overrightarrow{\mathbf{A}}_{(+\Omega)} \otimes \overrightarrow{\mathbf{A}}_{(-\Omega)} \text {, }
\end{aligned}
$$

Utilizamos a operação de mudança de base para levar $\mathbf{G}_{(s / a)}(\kappa)$ para a base das bandas laterais, pois é nessa base que será feito o tratamento da próxima seção. Por questão de simplicidade, vamos abreviar a notação do ganho na base simétrica e antissimétrica para $\mathbf{G}(\kappa)$. 


\subsection{Volta dos feixes na cavidade}

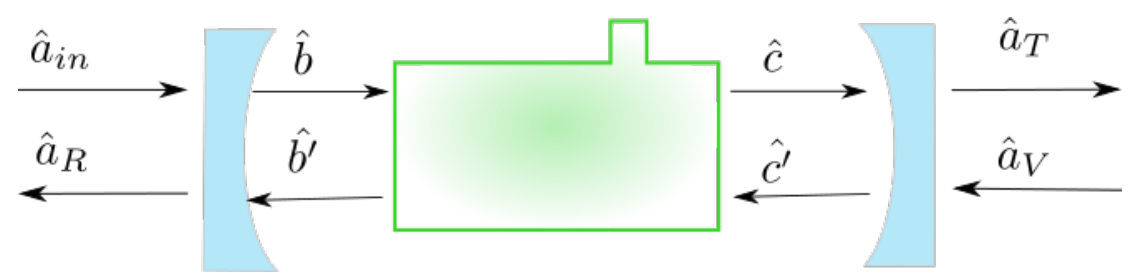

Figura 3.10: Operadores referentes a um único modo da cavidade em uma volta completa. Em que $\hat{a}_{i n}$ representa um modo do campo entrando na cavidade, $\hat{b}, \hat{c}, \hat{c^{\prime}}, \hat{b}^{\prime}$ são os campos intracavidade, $\hat{a}_{R}$ é o campo refletido, $\hat{a}_{T}$ é o campo transmitido e $\hat{a}_{V}$ é o campo de vácuo.

Nessa seção utilizamos a técnica desenvolvida em [53] para encontrar as flutuações dos modos do bombeio, prova e conjugado refletidos por um OPO com meio de ganho $\chi^{3}$ na base dos operadores simétrico e antissimétrico. Para iniciar essa análise é preciso entender o que ocorre com um modo individual do campo em uma volta na cavidade, e a figura 3.10 representa as transformações sofridas por um modo individual do campo em sua propagação por cavidade linear, como a cavidade utilizada no experimento desse trabalho. A transformação de uma cavidade em anel para uma linear é trivial, a cavidade em anel é equivalente a uma cavidade linear se seu meio de ganho possui dobro do tamanho do meio de ganho da cavidade linear. Nessa figura, o operador $\hat{a}_{\text {in }}$ representa um modo do campo entrando na cavidade, $\hat{b}$ o campo transmitido para o interior da cavidade, $\hat{c}$ o campo após a primeira passagem pelo meio de ganho, $\hat{c}^{\prime}$ o campo refletido pelo segundo espelho da cavidade, $\hat{b}^{\prime}$ o campo após a segunda passagem pelo cristal, $\hat{a}_{R}$ é o campo refletido pela cavidade, $\hat{a}_{T}$ é o campo transmitido pelo espelho de saída e $\hat{a}_{V}$ é o campo de vácuo. Os parâmetros $r$ e $t$ representam os coeficientes de reflexão e transmissão do espelho de entrada, $r^{\prime}$ e $t^{\prime}$ representam reflexão e transmissão do espelho de saída.

As equações que relacionam os campos no interior da cavidade aos campos no exterior da cavidade são dadas por:

$$
\begin{array}{ll}
\hat{a}_{R}=r \hat{a}_{i n}+t \hat{b}^{\prime}, & \hat{b}=t \hat{a}_{i n}-r \hat{b}^{\prime}, \\
\hat{a}_{T}=r^{\prime} \hat{a}_{\nu}+t^{\prime} \hat{c}, & \hat{c}^{\prime}=t^{\prime} \hat{a}_{\nu}-r^{\prime} \hat{c} .
\end{array}
$$

A evolução livre dos campos adiciona uma fase de $e^{i k L}$ em que $\mathrm{k}$ é o número de onda para a frequência do campo em análise e L é a distância percorrida (o comprimento da cavidade):

$$
\hat{c}=e^{i k L} \hat{b}, \quad \hat{b}^{\prime}=e^{-i k L} \hat{c}^{\prime} .
$$

Esse conjunto de equações se refere a um modo individual do campo. No entanto, podemos considerar um vetor que contenha os operadores que representam cada modo do campo e 
reescrever as equações 3.52 e 3.53 em forma matricial usando a mesma notação descrita na equação 3.43 :

$$
\begin{array}{ll}
\overrightarrow{\mathbf{A}}_{R}=\mathbf{R} \overrightarrow{\mathbf{A}}_{i n}+\mathbf{T} \overrightarrow{\mathbf{B}}^{\prime}, & \overrightarrow{\mathbf{B}}=\mathbf{T} \overrightarrow{\mathbf{A}}_{i n}-\mathbf{R} \vec{B}^{\prime} \\
\overrightarrow{\mathbf{A}}_{T}=\mathbf{R}^{\prime} \overrightarrow{\mathbf{A}}_{\nu}+\mathbf{T}^{\prime} \overrightarrow{\mathbf{C}}, & \overrightarrow{\mathbf{C}^{\prime}}=\mathbf{T}^{\prime} \overrightarrow{\mathbf{A}}_{\nu}-\mathbf{R}^{\prime} \overrightarrow{\mathbf{C}}
\end{array}
$$

onde as matrizes $\mathbf{R}, \mathbf{R}^{\prime}$, T e $\mathbf{T}^{\prime}$ são responsáveis por fazer as transformações de reflexão e transmissão pelos espelhos na base das bandas superior e inferior, e são dadas por :

$$
\begin{aligned}
& \mathbf{R}=\operatorname{diag}\left(r^{(0)} \quad r^{(0)} \quad \ldots \quad r^{(0)} r^{(0)} \quad \ldots\right), \\
& \mathbf{T}=\operatorname{diag}\left(t^{(0)} \quad t^{(0)} \quad \ldots \quad t^{(0)} t^{(0)} \ldots\right), \\
& \mathbf{R}^{\prime}=\operatorname{diag}\left(r^{\prime(0)} \quad r^{\prime(0)} \quad \ldots \quad r^{(0)} \quad r^{\prime(0)} \quad \ldots\right), \\
& \mathbf{T}^{\prime}=\operatorname{diag}\left(\begin{array}{llllll}
t^{\prime(0)} & t^{\prime(0)} & \ldots & t^{\prime(0)} & t^{\prime(0)} & \ldots
\end{array}\right) .
\end{aligned}
$$

Levando em conta o ganho no cristal, podemos reescrever as Equações 3.54 como:

$$
\overrightarrow{\mathbf{C}}=e^{-i \varphi} \mathbf{G}(\kappa) \overrightarrow{\mathbf{B}}, \quad \overrightarrow{\mathbf{B}}^{\prime}=e^{-i \varphi} \mathbf{G}(\kappa) \overrightarrow{\mathbf{C}}^{\prime},
$$

onde:

$$
\begin{aligned}
\varphi= & \gamma^{\prime} \operatorname{diag}\left(\Delta^{(0)} \pm \Omega^{\prime}, \quad-\left(\Delta^{(0)} \pm \Omega^{\prime}\right), \quad \Delta^{(1)} \pm \Omega^{\prime}, \quad-\left(\Delta^{(1)} \pm \Omega^{\prime}\right),\right. \\
& \left.\Delta^{(2)} \pm \Omega^{\prime}, \quad-\left(\Delta^{(2)} \pm \Omega^{\prime}\right)\right)
\end{aligned}
$$

é a matriz diagonal que introduz a fase da evolução livre no sistema. O parâmetro $\gamma^{\prime}=$ $t^{2} / 2+t^{\prime 2} / 2$ representa as perdas totais do sistema, $\Delta^{(n)}$, a dessintonia normalizada pela largura de banda e $\Omega^{\prime}$ a frequência de análise normalizada pela largura de banda.

Por meio de manipulações algébricas das equações 3.55 e 3.56 podemos encontrar uma solução para $\overrightarrow{\mathbf{A}_{R}}$ :

$$
\overrightarrow{\mathbf{A}}_{R}=\mathbf{R}_{\kappa} \overrightarrow{\mathbf{A}}_{i n}+\mathbf{T}_{\kappa} \overrightarrow{\mathbf{A}}_{\nu} .
$$

Onde as matrizes $\mathbf{R}_{\kappa}$ e $\mathbf{T}_{\kappa}$ são dadas por:

$$
\begin{aligned}
\mathbf{R}_{\kappa} & =\mathbf{R}-\mathbf{T} e^{-i \phi} \mathbf{G}(\kappa) \mathbf{R}^{\prime} e^{-i \phi} \mathbf{G}(\kappa) \mathbf{D}(\kappa) \mathbf{T} \\
\mathbf{T}_{\kappa} & =\mathbf{T} e^{-i \phi} \mathbf{G}(\kappa) \mathbf{T}^{\prime}+\mathbf{T} e^{-i \phi} \mathbf{G}(\kappa) \mathbf{R}^{\prime} e^{-i \phi} \mathbf{G}(\kappa) \mathbf{D}(\kappa) \mathbf{R} e^{-i \phi} \mathbf{G}(\kappa) \mathbf{T}^{\prime}
\end{aligned}
$$

Em que a matriz $\mathbf{D}(\kappa)$ é dada por:

$$
\mathbf{D}(\kappa)=\left(1-\mathbf{R} e^{-i \phi} \mathbf{G}(\kappa) \mathbf{R}^{\prime} e^{-i \phi} \mathbf{G}(\kappa)\right)^{-1} .
$$


Observe que encontrar a matriz $\overrightarrow{\mathbf{A}}_{R}$ equivale a encontrar a evolução de cada um dos nossos operadores após dar uma volta completa na cavidade.

Por fim, reescrevemos o vetor $\overrightarrow{\mathbf{A}}_{R}$ na base simétrica e antissimétrica:

$$
\overrightarrow{\mathbf{A}}_{R(s / a)}=\Lambda \overrightarrow{\mathbf{A}}_{R}
$$

\subsubsection{Matriz de covariância}

O tratamento apresentado na subseção 3.5 descarta termos de flutuação de ordem superior à um. Isso equivale a uma aproximação para um estado gaussiano, ou seja, estados que são completamente caracterizados por seus momentos de segunda ordem, ou de forma equivalente, por sua matriz de covariância conforme apresentado na seção 2.5.

Um vetor $\overrightarrow{\mathbf{X}}_{(s / a)}$ se relaciona ao vetor $\overrightarrow{\mathbf{A}}_{(s / a)}$ na base simétrica e antisimétrica por:

$$
\mathbf{X}_{(s / a)}^{\vec{N}}=\overrightarrow{\mathbf{N}} \overrightarrow{\mathbf{A}}_{(s / a)}
$$

em que $\overrightarrow{\mathrm{N}}$ é dada por:

$$
\overrightarrow{\mathbf{N}}=\frac{1}{2}\left(\begin{array}{cccccc}
\mathbf{B}_{4 x 4} & 0 & 0 & 0 & 0 & 0 \\
0 & \mathbf{B}_{4 x 4} & 0 & 0 & 0 & 0 \\
0 & 0 & \mathbf{B}_{4 x 4} & 0 & 0 & 0 \\
0 & 0 & 0 & \mathbf{B}_{4 x 4} & 0 & 0 \\
0 & 0 & 0 & 0 & \mathbf{B}_{4 x 4} & 0 \\
0 & 0 & 0 & 0 & 0 & \mathbf{B}_{4 x 4}
\end{array}\right), \quad \mathbf{B}_{4 x 4}=\left(\begin{array}{cc}
1 & 1 \\
-i & i
\end{array}\right) \text {. }
$$

Então o operador de quadratura $\overrightarrow{\mathbf{X}}_{R}$ dos campos refletidos pelo OPO satisfaz a relação $\overrightarrow{\mathbf{X}}=$ $\tilde{\mathbf{R}}_{\kappa} \overrightarrow{\mathbf{X}}_{i n}+\tilde{\mathbf{T}}_{\kappa} \overrightarrow{\mathbf{X}}_{\nu}$. Onde $\tilde{\mathbf{R}}_{\kappa}=\mathbf{N R}_{\kappa} \mathbf{N}^{-1}$ e $\tilde{\mathbf{R}}_{\kappa}=\mathbf{N T}_{\kappa} \mathbf{N}^{-1}$. Substituindo $\overrightarrow{\mathbf{X}}_{R}$ na equação 2.35, encontramos a forma da matriz de covariância dos campos refletidos pelo OPO:

$$
\mathbf{V}_{R}=\tilde{\mathbf{R}}_{\kappa} \mathbf{V}_{i n} \tilde{\mathbf{R}}_{\kappa}^{-1}+\tilde{\mathbf{T}}_{\kappa} \mathbf{V}_{\nu} \tilde{\mathbf{T}}_{\kappa}^{-1}
$$

em que $\mathbf{V}_{\text {in }}$ é a matriz de covariância dos campos de bombeio, prova e conjugado entrando na cavidade e $\mathbf{V}_{\nu}$ é a matriz de covariância dos modos do vácuo entrando na cavidade. Como tanto o ruído do vácuo como o laser do bombeio são campos coerentes, temos que $\mathbf{V}_{\nu}=\mathbf{V}_{\text {in }}=\mathbf{I}$. Nas próximas seções será feita a análise dos resultados obtidos para as variâncias e correlações assim como testes de emaranhamento baseados nos critérios de Duan e critério de positividade sobre transposição parcial (PPT) para o OPO duplamente ressonante. 


\subsubsection{Pureza}

Como pode ser visto em [54] a pureza é uma grandeza que mede a independência de subespaços e é definida por $\wp=\operatorname{Tr} \hat{\rho}^{2}$. Quando $\wp=1$ então o estado definido por $\hat{\rho}$ é puro e podemos descrever o estado quântico do sistema por um vetor no espaço de Hilbert. Quando $\wp<1$ o estado é uma mistura estatística de estados puros e perdemos parte da informação do sistema. Neste caso, o estado só pode ser descrito por meio da matriz densidade $\hat{\rho}$.

Um estado com mistura pode surgir devido a uma mistura estatística de estados puros ou devido a emaranhamento, em geral, não é fácil separar um caso do outro. No entanto, quando um subsistema de um sistema global puro possui $\wp<1$ podemos concluir que esse subsistema está emaranhado ao restante do sistema.

Para estados gaussianos, a pureza pode ser escrita em termos da matriz de covariância do sistema, pois essa contém toda a informação dos momentos de segunda ordem. Sendo $|\mathbf{V}|$ o determinante da matriz de covariância, a pureza pode ser definida por [55]:

$$
\wp=\frac{1}{\sqrt{|\mathbf{V}|}} .
$$

\subsection{OPO duplamente ressonante na base simétrica e antis- simétrica}

Nessa seção iremos analisar as variâncias e correlações dos campos refletidos pelo OPO por meio da análise gráfica dos termos da equação 3.68. Essa análise é feita em função de $\sigma$, potência de bombeio entrando na cavidade normalizada pela potência limiar $\sigma=P_{0 i n} / P_{0 t h}$, para potências entre $\sigma=1$ e $\sigma=4$. Por simplicidade indicaremos os parâmetros referentes aos campos de bombeio, prova e conjugado pelos subíndices 0,1 e 2, respectivamente. Analisaremos a situação em que a cavidade é ressonante com os três campos, ou seja, $\Delta_{0}=\Delta_{1}=\Delta_{2}=0$, e a frequência de análise relativa a largura de banda, $\Omega^{\prime}=\Omega / \delta \omega_{c}$, é de $\Omega^{\prime}=0,5$. A perda por transmissão para os feixes de prova e conjugado é dada por $\gamma_{1}=\gamma_{2}=\sqrt{0.4}$ e as perdas espúrias por $\mu_{1}=\mu_{2}=0$. Para o OPO duplamente ressonante o feixe de bombeio não cavita, esse fato é equivalente a situação em que todo o feixe de bombeio é transmitido pelos espelhos $\gamma_{0}=1 \mathrm{e}$ $\mu_{0}=0$.

Para melhor visualizarmos a matriz de covariância iremos dividi-la em blocos:

$$
\mathbf{V}_{s / a}=\left(\begin{array}{cc}
\mathbf{V}_{s}^{(012)} & \mathbf{C}_{s / a}^{(012)} \\
\mathbf{C}_{s / a}^{(012) T} & \mathbf{V}_{a}^{(012)}
\end{array}\right)
$$

onde os índices 0, 1, 2 se referem aos campos de bombeio, prova e conjugado, e os blocos apresentados relacionam esses campos da seguinte forma: $\mathbf{V}_{s}^{(012)}$ contém todas a variâncias e correlações na base simétrica, $\mathbf{V}_{a}^{(012)}$ contém variâncias e correlações na base antissimétrica, e 
os blocos $\mathbf{C}_{s / a}^{(012)}$ contém todas as correlações entre os campos na base simétrica e antissimétrica. A análise dos resultados não irá mencionar termos de $\mathbf{V}_{a}^{(012)}$, pois essa matriz só difere de $\mathbf{V}_{s}^{(012)}$ por uma rotação de $\pi / 2$, mas apresenta a mesma informação já obtida na parte simétrica [34].

Nas equações 3.42 podemos ver que a taxa de variação das flutuações dos campos de prova e conjugado no interior da célula de rubídio são totalmente análogas. Sendo assim, a variância do feixe de prova é igual à variância para o feixe conjugado e correlações do bombeio com os feixes de prova ou conjugado são iguais. Portanto omitiremos resultados de variância do conjugado e correlações entre os feixes de bombeio e conjugado.

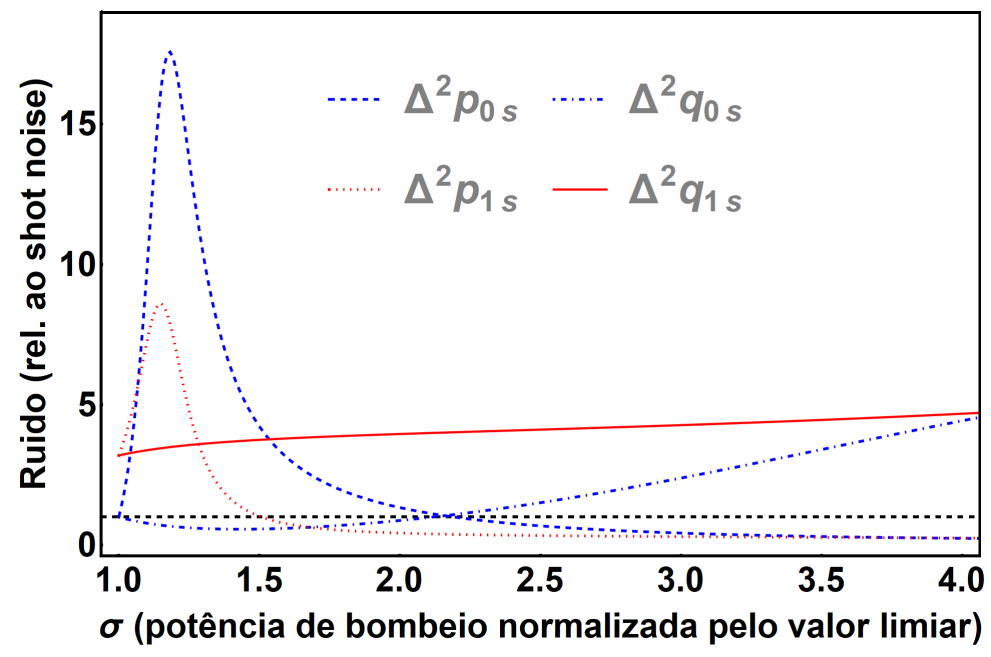

Figura 3.11: Variâncias da parte simétrica das quadraturas de amplitude e fase para os feixes de prova e conjugado em função da potência do bombeio $(\Omega=0,5) . \Delta^{2} p_{0 s}$ - variância da amplitude simétrica para o modo do bombeio; $\Delta^{2} p_{1 s}$ - variância da amplitude simétrica para o modo de prova; $\Delta^{2} q_{0 s}$ - variância da fase simétrica para o modo do bombeio; $\Delta^{2} q_{1 s}$ - variância da fase simétrica para o modo de prova.

Na figura 3.11 observamos as variâncias da parte simétrica das quadraturas de amplitude e fase para os feixes de bombeio e prova. Vamos começar nossa análise vendo como se comportam as variâncias do campo de bombeio. Em geral, os trabalhos teóricos para o OPO são feitos abaixo do limiar e por isso o bombeio é considerado um campo clássico. No entanto, como estamos trabalhando acima do limiar ocorre depleção do bombeio, consideramos o bombeio como um campo quântico, e esse tipo de tratamento para o bombeio do DROPO é inédito. As quadraturas amplitude e fase do feixe de bombeio apresentam um comportamento muito interessante. Na região próxima ao limar a quadratura de amplitude do bombeio $\left(\Delta^{2} p_{0 s}\right)$ apresenta um pico de excesso de ruído $1<\sigma \leq 2,15$ enquanto a quadratura de fase $\left(\Delta^{2} q_{0 s}\right)$ apresenta compressão de ruído. Para $\sigma \approx 2,15$, o campo de bombeio se torna quase coerente mas as variâncias ainda são maiores que o ruído quântico padrão $\left(\Delta^{2} p_{0 s}=\Delta^{2} q_{0 s}=1,06\right)$. Para $\sigma>2,15$, o comportamento das quadraturas $\Delta^{2} p_{0 s}$ e $\Delta^{2} q_{0 s}$ se inverte, ou seja, $\Delta^{2} p_{0 s}$ apresenta compressão de ruído e $\Delta^{2} q_{0 s}$ apresenta excesso de ruído. Passando à análise do campo de prova, 
vemos que a quadratura de fase do feixe de prova $\left(\Delta^{2} q_{1 s}\right)$ sempre apresenta excesso de ruído. Sua quadratura conjugada $\left(\Delta^{2} p_{1 s}\right)$ apresenta um pico de excesso de ruído nas proximidades do limiar que cai abruptamente até $\sigma>1,5$, onde temos compressão de ruído.

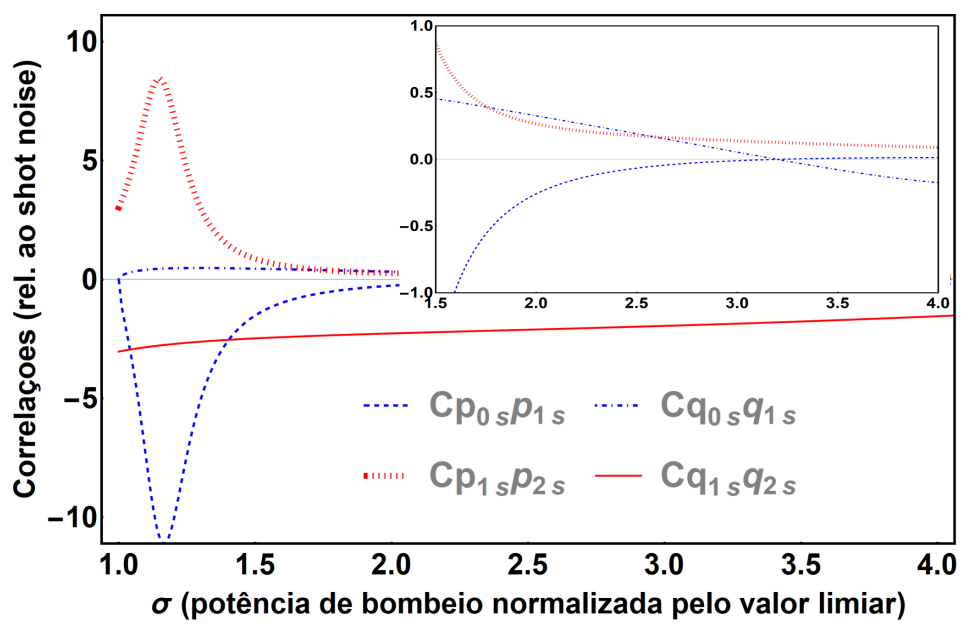

Figura 3.12: Parte simétrica das correlações entre bombeio, prova e conjugado $(\Omega=0,5): C p_{0 s} p_{1 s}$ - Correlação entre as quadraturas de amplitude simétricas do bombeio e prova; $C q_{0 s} q_{1 s}$ - Correlações entre as quadraturas de fase simétricas dos modos de bombeio e complementar; $C p_{1 s} p_{2 s}$ - Correlação entre as quadraturas de amplitude simétricas dos modos de prova e conjugado; $C q_{1 s} q_{2 s}$ - Correlações entre as quadraturas de fase simétricas dos modos de prova e conjugado.

A figura 3.12 apresenta a parte simétrica das correlações não nulas entre bombeio, prova e conjugado. Próximo ao limiar vemos um pico de correlação entre as amplitudes dos campos de prova e conjugado $\left(C p_{1 s} p_{2 s}\right)$ que cai praticamente a zero após $\sigma=3$ mas ainda se mantém positiva. A correlação entre as amplitudes dos campos de bombeio e prova, $\left(C p_{0 s} p_{1 s}\right)$, apresenta um pico de anticorrelação próximo ao limiar de oscilação, essa anticorrelação chega a zero em $\sigma \approx 3,2$ e se torna positiva mas praticamente nula para $3,2<\sigma<4$. Existe uma pequena correlação entre as quadraturas fase dos campos de bombeio e prova $\left(C q_{0 s} q_{1 s}\right)$ positiva para $\sigma<3,2$, e negativa no intervalo entre $3,2<\sigma<4$. A correlação $C q_{1 s} q_{2 s}$ é sempre negativa no intervalo analisado $1<\sigma<4$.

A figura 3.13 apresenta os resultados para as correlações não nulas entre as quadraturas da base simétrica e antissimétrica. As correlações entre a amplitude simétrica do campo de prova e fase antissimétrica do campo de bombeio, $C p_{1 a} q_{0 s}$, e $C p_{1 s} q_{0 a}$ são aproximadamente nulas até $\sigma=2$, após esse ponto $C p_{1 a} q_{0 s}$ cresce de forma abrupta, enquanto $C p_{1 s} q_{0 a}$ decresce de forma abrupta. Para $C p_{0 s} q_{1 a}$ temos um pequeno pico de anticorrelação nas proximidades do limiar, para potências maiores que $\sigma=2$ temos uma pequena correlação. Para $C p_{0 a} q_{1 s}$ vemos um pico de correlação nas proximidades do limiar, em $\sigma=2$ o comportamento muda e passamos a ver correlações positivas. 


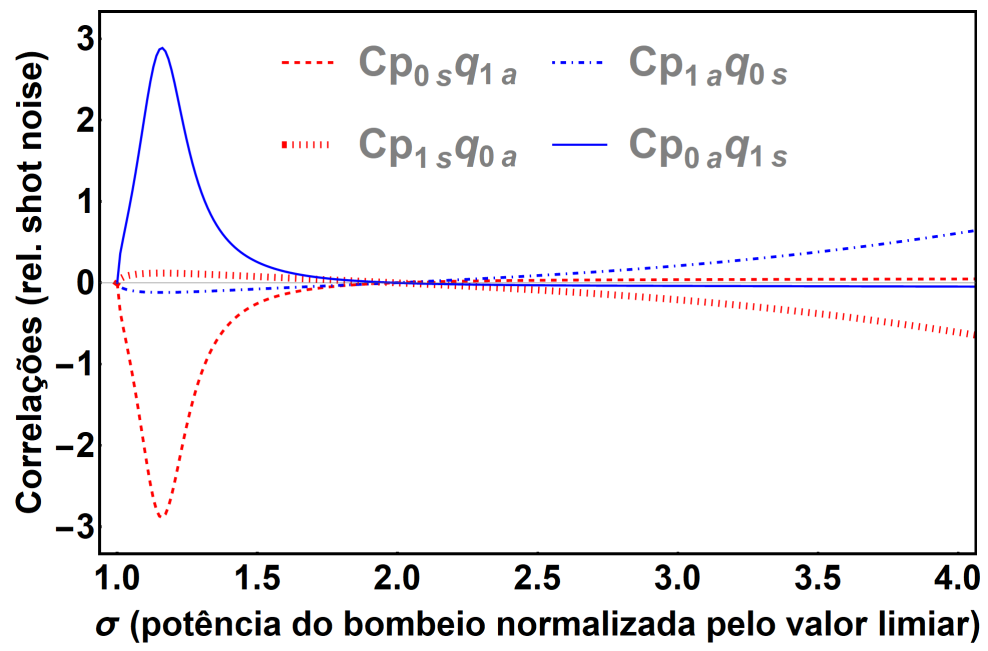

Figura 3.13: Correlações não nulas entre as quadraturas da base simétrica e antissimétrica em função da potência do bombeio $(\Omega=0,5)$. $C p_{0 s} q_{1 a}$ - Correlação entre a quadratura de amplitude simétrica do bombeio e a quadratura de fase antissimétrica do prova; $C p_{1 a} q_{0 s}$ - Correlação entre a quadratura de amplitude antissimétrica do conjugado e a quadratura de fase simétrica do bombeio; $C p_{1 s} q_{0 a}-$ Correlação entre a quadratura de amplitude simétrica do prova e a quadratura de fase antissimétrica do modo de bombeio; $C p_{0 a} q_{1 s}$ - Correlação entre a quadratura de amplitude antissimétrica do bombeio e a quadratura de fase simétrica do prova.

Na figura 3.14 (a) apresentamos o comportamento da pureza de subsistemas que incluem as partes simétrica e antissimétrica de cada um dos campos que compõe o sistema em estudo. A curva azul pontilhada representa a pureza total, $\wp_{\text {Total }}$, calculada a partir da matriz de covariância completa do nosso sistema, com informação dos campos de bombeio, prova e conjugado, mas sem considerar perdas espúrias. Utilizamos essa quantidade como teste de sanidade do modelo e obtivemos $\wp_{\text {Total }}=1$, como era de se esperar de um estado que só sofreu transformações unitárias. As demais curvas representam a pureza de cada um dos campos separadamente: curva em laranja para o bombeio, $\wp_{\text {sBombeio }}$, e curva verde para prova e conjugado, $\wp_{\text {Prova }}=\wp_{\text {Conjugado }}$. Essa análise é realizada através do traço parcial de cada um dos subsistemas. Percebemos pelo comportamento das curvas analisadas que todos os três campos apresentam pureza menor que 1, o que indica que existe mais informação no sistema completo dos três campos do que pode ser obtida da análise dos três campos de forma individual. Percebemos ainda que na região $1<\sigma<2$ a pureza dos campos de prova e bombeio apresentam um vale que coincide com a região em que temos os picos nas quadraturas de amplitude dos campos de bombeio e prova, dessa forma, percebemos que esses picos de excesso de ruído das variâncias juntamente com as fortes correlações entre os campos na base simétrica e antissimétrica são um indício de que o emaranhamento é mais forte nessa região. A medida que nos afastamos da região dos picos a pureza aumenta mas não atinge valor unitário. Esse resultado é coerente com o estudo das variâncias e correlações que foi feito até aqui. Podemos destacar que em torno de $\sigma=2$, 1 , onde as variâncias de amplitude e fase do bombeio se cruzam em um ruído de 1,06 e as correlações de 
forma geral são fracas, a pureza do bombeio sobe bastante chegando a $\wp_{\text {Bombeio }}=0,89$ e ainda subindo um pouco mais para maiores valores de $\sigma$ mas nunca atingindo 1 . Outro ponto que podemos destacar é que em $\sigma=3,2$ as correlações de amplitude e fase simétrica do bombeio se cruzam em zero, no entanto, ainda temos correlações entre as partes simétrica e antissimétricas do bombeio e prova diferentes de zero de forma que a pureza ainda é menor que 1.

A figura 3.14 (b) apresenta a pureza da parte simétrica dos subsistemas de bombeio, $\wp_{\text {sBombeio }}$, prova, $\wp_{\text {sProva }}$, e conjugado, $\wp_{\text {sConjugado }}$ e a pureza do subsistema composto pelos modos simétricos dos campos de bombeio, prova e conjugado, $\wp_{\text {stotal }}$. Em toda a região em que $\wp_{\text {stotal }}<1$, temos a parte simétrica do sistema emaranhada à parte antissimétrica. Para o nosso sistema, essa afirmação é verdadeira nas regiões entre $1<\sigma<1,70$ e $2,5<\sigma<4$.

(a)

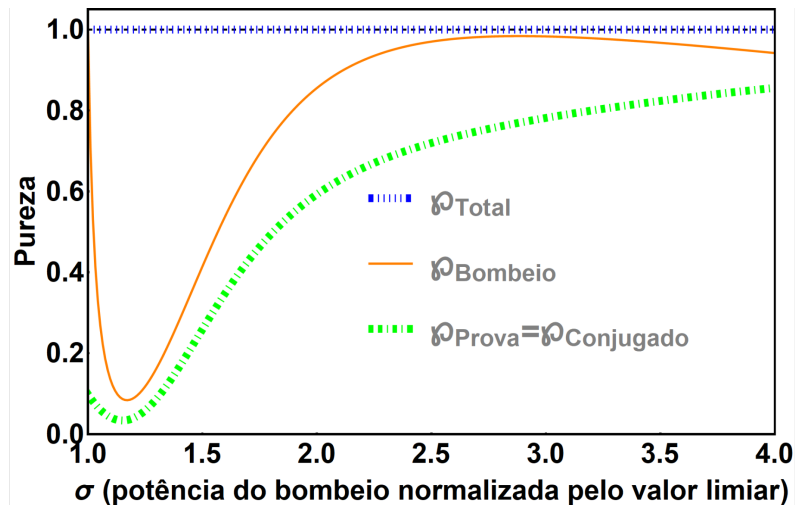

(b)

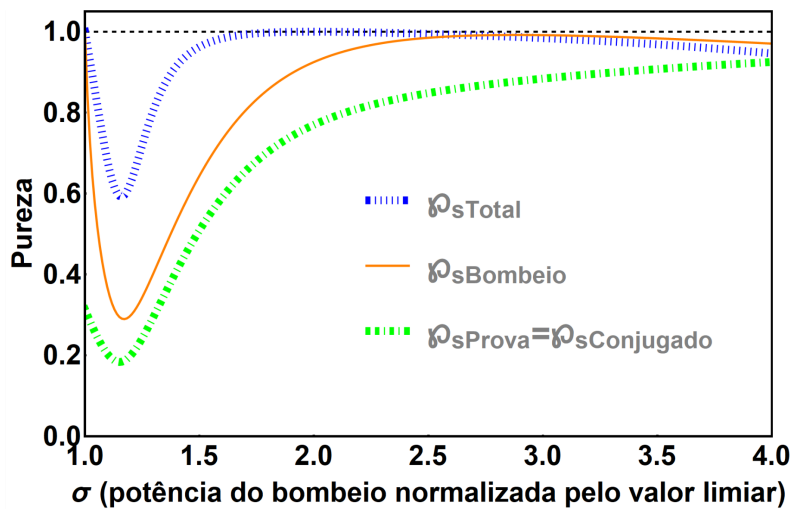

Figura 3.14: Pureza dos subsistemas formados pelas partes simétrica e antissimétrica do bom-

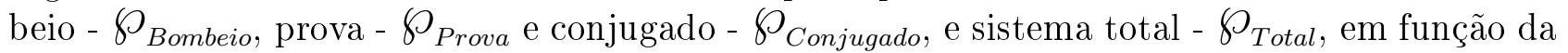
potência de bombeio normalizada para frequência de análise $(\Omega=0,5)$. (b) Pureza da parte simétrica dos subsistemas - bombeio - $\wp_{\text {sBombeio }}$, prova - $\wp_{\text {sProva }}$, conjugado - $\wp_{\text {sConjugado }}$ e sistema total simétrico - $\wp_{\text {sTotal, }}$ em função da potência de bombeio normalizada para frequência de análise $(\Omega=0,5)$.

\subsection{OPO duplamente ressonante na base soma e diferença}

É importante estudar as variâncias e correlações na base da soma e subtração dado que algumas testemunhas de emaranhamento as usam como referência, como por exemplo, o critério de Duan [46] que será utilizado aqui para testar o emaranhamento entre os campos de prova e conjugado. Sendo assim, consideramos as variáveis tipo EPR formadas pelas combinações lineares dos modos de prova e conjugado dadas por:

$$
\hat{p}_{ \pm}=\frac{\hat{p}_{1 s} \pm \hat{p}_{2 s}}{\sqrt{2}}, \quad \hat{q}_{ \pm}=\frac{\hat{q}_{1 s} \pm \hat{q}_{2 s}}{\sqrt{2}} .
$$


Faremos nossa análise considerando apenas a base simétrica pois a parte antissimétrica fornece um resultado equivalente porém com uma rotação de $\pi / 2$ entre as quadraturas.

(a)

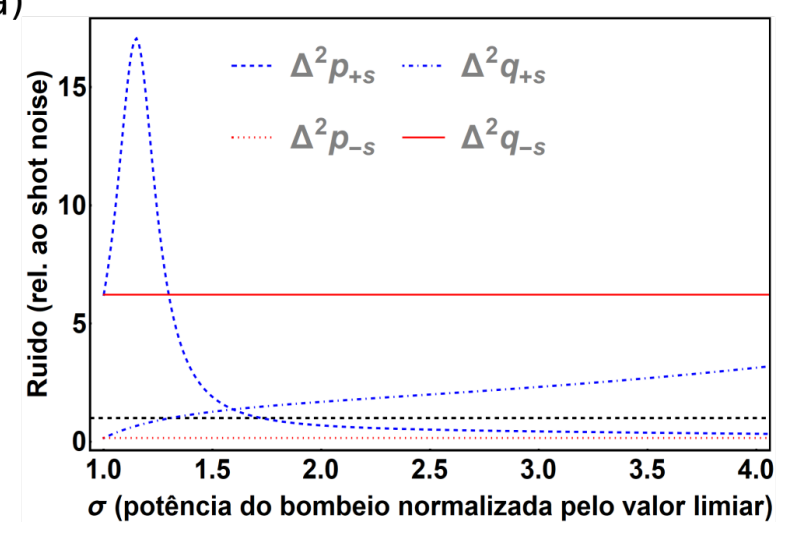

(b)

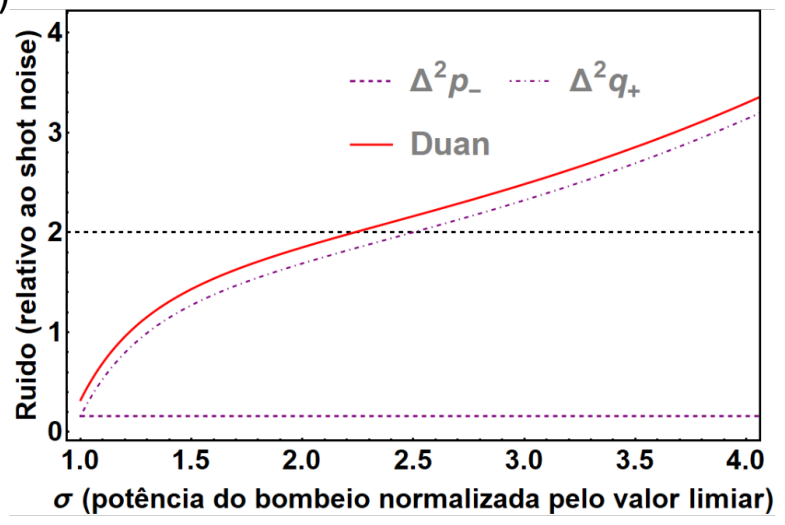

Figura 3.15: (a) Variâncias na base da soma e subtração em função da potência do bombeio $(\Omega=0,5)$; (b) teste de Duan $(\Omega=0,5) . \Delta p_{+s}$ - Variância da amplitude simétrica do modo da soma; $\Delta^{2} q_{+s}-$ Variância da fase simétrica do modo da soma; $\Delta p_{-s}$ - Variância da amplitude simétrica do modo da diferença; $\Delta^{2} q_{-s}$ - Variância da fase simétrica do modo da diferença.

Na figura 3.15(a) observamos o resultado obtido para as variâncias dos modos da soma e subtração em função da potência do bombeio. As variâncias do modo da diferença $\Delta^{2} p_{-s}$, $\Delta^{2} q_{-s}$ não dependem de $\sigma$, ou seja, esse subespaço se encontra desacoplado do restante do sistema. Dado que quando criamos um fóton no modo de prova também criamos um fóton no modo conjugado, já era esperado que, na ausência de perdas espúrias, a diferença entre as variâncias das quadraturas de amplitude de prova e conjugado fosse nula em frequência de análise zero implicando em um compressão de ruído, caso estivéssemos medindo em frequência de análise zero essa compressão seria perfeita. Se temos compressão de ruído que independe dos demais subsistemas na quadratura da diferença das amplitude, isso implica em um excesso de ruído que independe dos demais subsistemas na quadratura de subtração das fase.

Ainda com base na figura 3.15 (a), analisamos os modos da soma dos campos de prova e conjugado. A variância da fase simétrica do modo da soma $\left(\Delta^{2} q_{+s}\right)$ apresenta compressão de ruído nas proximidades do limiar, tal comportamento é acompanhado por um pico de excesso de ruído em sua quadratura conjugada, $\Delta^{2} p_{+s}$. Após $\sigma=1,7$ a quadratura $\Delta^{2} p_{+s}$ passa a apresentar compressão de ruído, que é compensado por um excesso de ruído em $\Delta^{2} q_{+s}$. Vemos compressão de ruído simultânea para as amplitudes da soma e diferença acima da potência $\sigma=1,7$, esse resultado é consequência da compressão nas quadraturas de amplitude de prova e conjugado a partir de $\sigma=1,5$, visto na 3.11, e da fraca correlação de amplitude na mesma região, vista na figura 3.12 .

A Figura 3.15(b) apresenta a análise gráfica do critério de Duan, que para o nosso sistema é descrito pela desigualdade

$$
\Delta^{2} \hat{p}_{-s}+\Delta^{2} \hat{q}_{+s} \geq 2
$$


cuja violação é uma condição suficiente para demonstrar emaranhamento. Assim, pela figura 3.15 vemos que prova e conjugado estão emaranhados para $\sigma<2,22$.

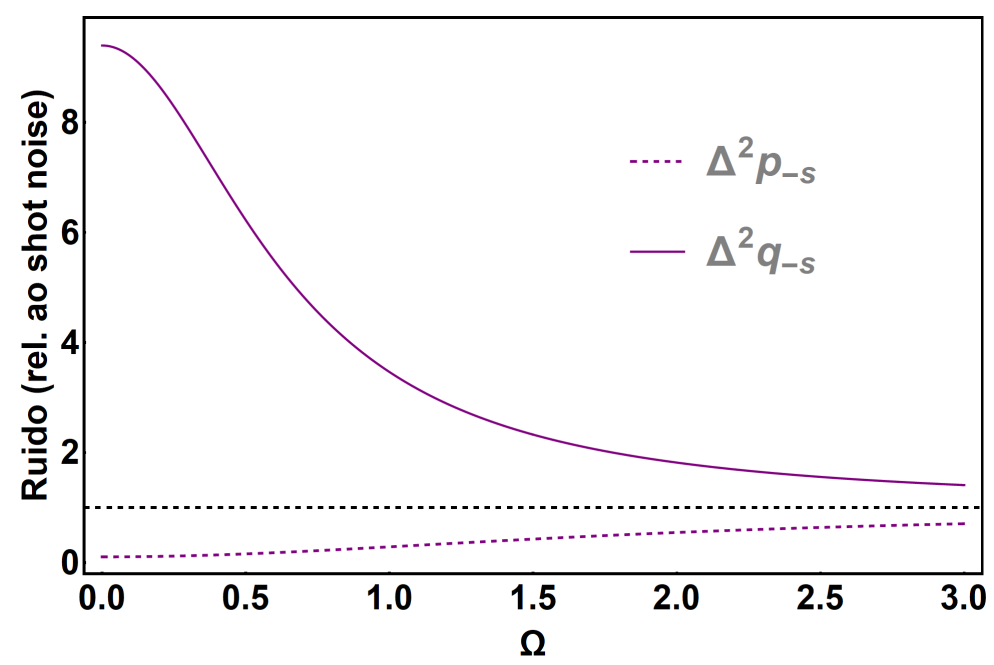

Figura 3.16: $\Delta^{2} p_{-s}$ - Variância da quadratura da diferença entre amplitudes dos modos de prova e conjugado em função da frequência de análise $\Omega ; \Delta^{2} q_{-s}$ - Variância da quadratura da diferença entre fases dos modos de prova e conjugado em função da frequência de análise.

A figura 3.16 apresenta as variância da parte simétrica da quadraturas da diferença das amplitudes $\left(\Delta^{2} p_{-s}\right)$ e diferença das fases $\left(\Delta^{2} q_{-s}\right)$ entre os modos de prova e conjugado em função da frequência de análise normalizada pela largura de banda, $\Omega$. Observa-se que a quadratura de amplitude $\left(\Delta^{2} p_{-s}\right)$ apresenta grande compressão de ruído nas proximidades de $\Omega=0$, essa compressão diminui a medida que nos afastamos do ponto zero. Por outro lado, a quadratura de fase apresenta um grande excesso de ruído, o que compensa a compressão no ruído de fase de forma a não violar o princípio da incerteza, também observamos a diminuição desse excesso de ruído a medida que nos afastamos de $\Omega=0$. Esse comportamento também foi observado para outras análises feitas para o OPO $\chi^{2}$. O que ocorre é que frequências de análise grandes $\Omega \gg 1$ são equivalentes a tempos de medida muito curtos, quando esse tempo é menor que o tempo de vida do fóton no interior da cavidade é mais provável que apenas um dos fótons tenha deixado a cavidade. Assim, a medida que nos afastamos da ressonância a correlação entre bombeio e prova diminui e os ruídos dos nossos campos tendem ao ruído quântico padrão dando a curva que descreve $\Delta^{2} p_{-s}$ um perfil lorentziano.

A figura 3.17 (a) apresenta as correlações não nulas da parte simétrica das quadraturas no espaço da soma, diferença e bombeio. Notamos que o espaço da diferença não apresenta correlação com os demais espaços, como já era esperado visto que possui pureza unitária e portanto encontra-se desacoplado dos demais subespaços. No entanto, existe uma grande anticorrelação entre a parte simétrica da soma das amplitudes entre prova e conjugado e amplitude do bombeio $\left(C p_{+s} p_{0 s}\right)$ nas proximidades do limiar. Essa correlação cai praticamente a zero a partir de $\sigma=2,5$. Também existe correlação entre a parte simétrica da fase do bombeio e parte 
(a)

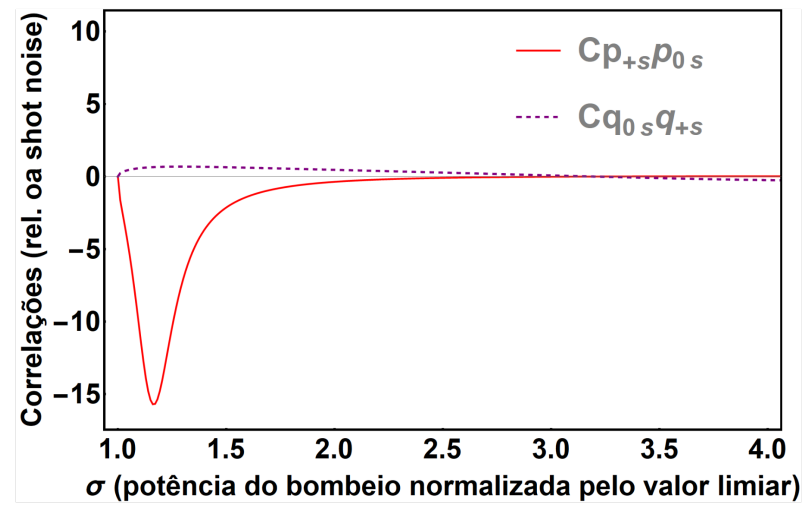

(b)

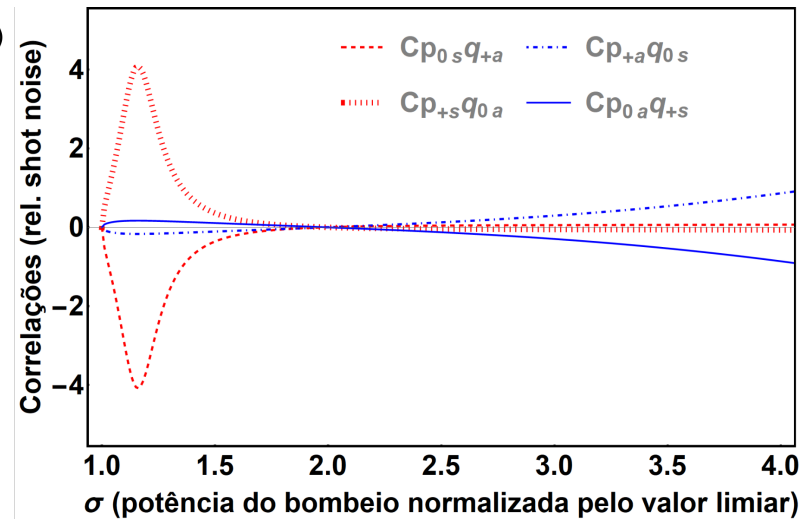

Figura 3.17: (a) Correlações não nulas da parte simétrica das quadraturas no espaço soma e bombeio em função da potência de bombeio $(\Omega=0,5)$. $C p_{+s} p_{0 s}$ - Correlação entre parte simétrica da soma das amplitudes entre prova e conjugado e amplitude do bombeio. $C q_{0 s} q_{+s}$ - Correlação entre a parte simétrica do bombeio e parte simétrica da soma das fases entre prova e conjugado. (b) Correlações não nulas entre as partes simétrica e antissimétrica das quadraturas em função da potência de bombeio $(\Omega=0,5)$. $C p_{0 s} q_{+a}$ - Correlação entre a quadratura antissimétrica de soma das fases com a quadratura simétrica de amplitude do bombeio; $C p_{+a} q_{0 s}$ - Correlação entre a quadratura antissimétrica da soma das amplitudes com a fase simétrica do bombeio; $C p_{+s} q_{0 a}$ - Correlação entre a quadratura soma das amplitudes com a fase do bombeio antissimétrico; $p_{0 a} C q_{+s}$ - Correlação entre a quadratura simétrica de soma das fases com a quadratura antissimétrica de amplitude do bombeio.

simétrica da soma das fases entre prova e conjugado $\left(C q_{0 s} q_{+s}\right)$. Esta correlação é positiva até uma potência de aproximadamente $\sigma=3,2$, e em seguida vê-se uma pequena anticorrelação até $\sigma=4$. O fato dos espaços da soma e bombeio estarem correlacionados indica que pode haver emaranhamento entre as quadraturas da soma de prova e conjugado e as quadraturas do bombeio.

A 3.17(b)apresenta os resultados não nulos das correlações entre as partes simétrica e antissimétrica das quadraturas no espaço da soma e bombeio. A correlação $C p_{0 s} q_{+a}$ apresenta um pequeno pico de anticorrelação nas proximidades do limiar e para $\sigma>1,5$ vemos correlação. Em $C p_{+a} q_{0 s}$ observamos uma correlação nula nas proximidades do limiar que cresce de forma rápida após esse ponto. A correlação $C p_{+s} q_{0 a}$ apresenta um pequeno pico de correlação próximo ao limiar e anticorrelação para $\sigma>1,5$. Em $C p_{0 a} q_{+s}$ vemos uma correlação nula nas proximidades do limiar que decresce rapidamente tornando as quadraturas em questão fortemente anticorrelacionadas.

Até esse momento, utilizamos o critério de Duan para investigar emaranhamento entre as quadraturas dos campos de prova e conjugado. Mas a análise da pureza dos subsistemas feita para a figura 3.18 mostra que o bombeio está emaranhado com o restante dos subsistemas. Sendo assim, nosso próximo passo será caracterizar o emaranhamento tripartite presente no sistema.

Vamos concluir a análise dos campos na base do bombeio, soma e diferença estudando a 
(a)

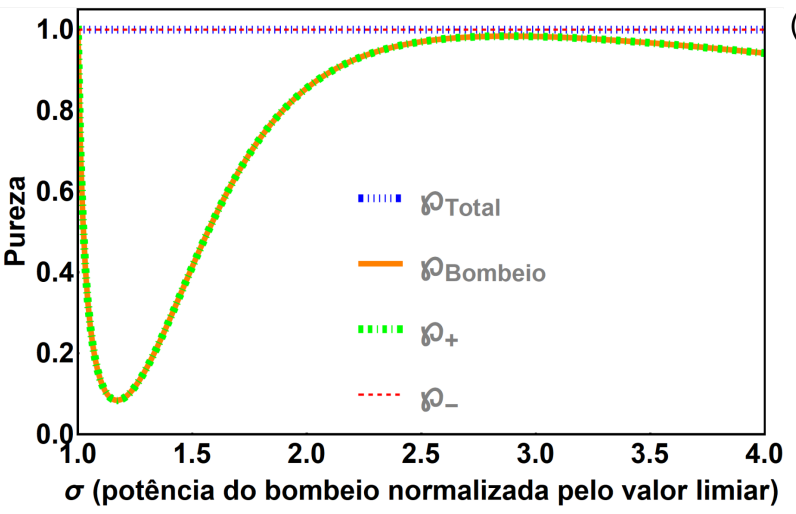

(b)

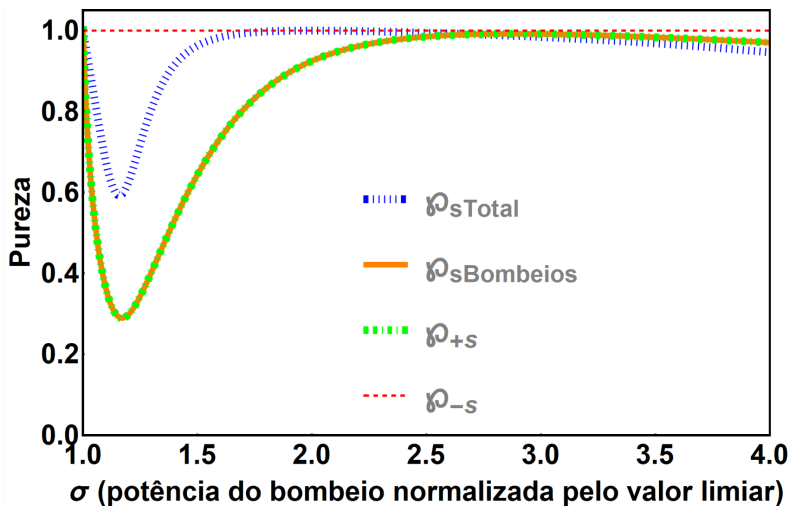

Figura 3.18: (a) Pureza dos subsistemas formados pelas partes simétrica e antissimétrica do bombeio - $\wp_{\text {Bombeio }}$, soma de prova e conjugado - $\wp_{+}$e diferença entre prova e conjugado - $\wp_{-}$, e sistema total - $\wp_{\text {Total }}$, em função da potência de bombeio normalizada para frequência de análise $(\Omega=0,5)$. (b) Pureza da parte simétrica dos modos do bombeio - $\oint_{\text {sBombeio }}$, soma de prova e conjugado - $\wp_{s+}$ e diferença entre prova e conjugado - $\wp_{s-}$ e sistema total simétrico $\wp_{\text {sTotal }}$, em função da potência de bombeio normalizada para frequência de análise $(\Omega=0,5)$.

pureza de cada um dos subsistemas descritos na figura 3.18. Observamos, na figura 3.18 (a), a pureza do subsistema da diferença $\wp_{-}=1$, o que significa que esse subsistema se encontra desacoplado dos demais, o que é consistente com as variâncias constantes das quadraturas de fase e amplitude (figura 3.15) e com a ausência de correlações desse subespaço com os demais (figura 3.15). Vemos também que $\wp_{\text {Bombeio }}=\wp_{+}$e que esses dois subsistemas sempre possuem pureza menor que 1 no intervalo analisado, chegando a $\wp_{\text {Bombeio }}=\wp_{+}=0,08$ em $\sigma=1.16$, ou seja, na região que coincide com os picos das variâncias de amplitude do espaço da soma e do espaço do bombeio além de apresentar anticorrelação forte entre as amplitudes simétricas dos subespaços do bombeio e soma. Como o sistema total é puro, a pureza menor que 1 mostra que existe emaranhamento entre o bombeio e o subsistema da soma dos campos de prova e conjugado.

A figura 3.18 (b) considera apenas os modos simétricos dos campos de bombeio, $\wp_{\text {sBombeio }}$, soma, $\wp_{+}$, diferença, $\wp_{-}$, dos campos de prova e conjugado. Observamos que o subsistema da diferença continua desemaranhados aos demais subsistemas, e que a pureza dos subsistemas do bombeio e da soma cresce. A pureza total, $\wp_{\text {sTotal }}$, mostra emaranhamento entre a parte simétrica e parte antissimétrica do sistema nas regiões $1<\sigma<1,73$ e $2,5<\sigma<4$.

\subsection{Teste de positividade sob transposição parcial (DROPO)}

\subsubsection{Tripartite}

O critério de Duan é um critério suficiente para caracterizar emaranhamento mas não necessário, ou seja, pode haver regiões de emaranhamento que não podemos localizar por meio desse critério 
[46]. Já o critério PPT é um critério necessário e suficiente para todas as possíveis decomposições 1 x N de um estado Gaussiano, onde $\mathrm{N}+1$ é o número total de modos emaranhados[48]. No caso de emaranhamento tripartite, testaremos as três bipartições 1 x 2 da base simétrica. A utilização do critério de Duan em conjunto com o critério PPT é interessante pois alguns protocolos de teletransporte necessitam de campos emaranhados que violem a desigualdade de Duan.

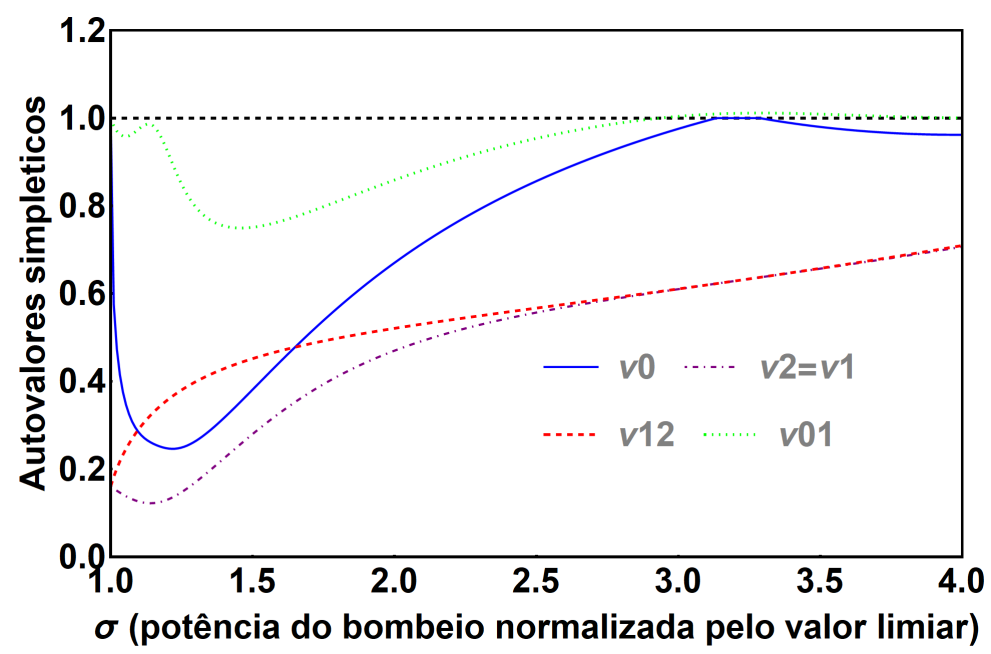

Figura 3.19: Teste de emaranhamento PPT $(\Omega=0,5) . \nu(\mathrm{n})$ - menor autovalor simplético obtido via transposição parcial de um modo da parte simétrica da matriz de covariância do OPO, onde os índices $\mathrm{n}=0,1,01,12$ representam os subsistemas referentes ao bombeio, prova, bombeio e prova, prova e conjugado respectivamente.

Nessa seção buscamos caracterizar o emaranhamento por meio do critério de positividade sob transposição parcial (PPT), apresentado na subseção 2.13.2. A figura 3.19 apresenta os autovalores simpléticos referentes a transposição parcial de diferentes subsistemas. Como visto na subseção 2.13.2 nas regiões em que o autovalor simplético é menor que 1 o subsistema transposto está emaranhado com o subsistema restante.

Também é interessante analisar o emaranhamento entre as diferentes bipartições do sistema. O autovalor simplético $\nu 0$ caracteriza o emaranhamento entre o bombeio e o restante do sistema. Pela análise gráfica vê-se que $\nu 0$ é menor que 1 para a região entre o limiar e $\sigma=3,1$ aproximadamente. A partir desse ponto temos uma pequena região em que há quebra de emaranhamento e em seguida a bipartição $\nu 0$ volta a apresentar emaranhamento $3,3<\sigma<4$. Na região em que há quebra de emaranhamento a correlação dentro da matriz simétrica entre os campos de bombeio com os campos sinal e complementar vai a zero (figura 3.12). No entanto, a correlação entre bombeio simétrico e sinal antissimétrico persiste (figura 3.13).

Os autovalores $\nu 1$ caracteriza a bipartição entre o prova e o restante do sistema, esse autovalor é igual à $\nu 2$ que caracteriza o emaranhamento entre conjugado e o restante do sistema. Vemos que essas duas bipartições estão emaranhadas com o restante do sistema entre a potência 
de limiar e $\sigma=4$.

O autovalor $\nu 12$ caracteriza emaranhamento entre prova e conjugado, essa bipartição encontrase emaranhada entre o limiar e $\sigma=4$. Vemos também que $\nu 12$ é maior que $\nu 1=\nu 2$ entre $1 \leq \sigma \leq 2,8$ indicando que o emaranhamento entre prova e conjugado se torna mais expressivo quando incluímos informações sobre o bombeio na análise dessa região. Na região $2,8 \leq \sigma \leq 4$ o autovalor simplético $\nu 12$ é praticamente igual à $\nu 1$ ou pouca coisa maior, essa também é a região onde temos uma quebra de emaranhamento entre bombeio e prova $(\nu 01 \geq 1)$ e onde $\nu 0$ está próximo ou se iguala a 1 . Ou seja, $\nu 12 \approx \nu 1$ na região onde o emaranhamento do bombeio com o restante do sistema é mais fraco ou inexistente.

A bipartição $\nu 01$ mede o emaranhamento entre bombeio e prova, que deve ser igual ao que se obtém para os feixes de bombeio e conjugado. Para essa última bipartição, temos emaranhamento entre o limiar e $\sigma \approx 2,8$. Vemos também que $\nu 01$ é maior que $\nu 0$ e maior que $\nu 1$ indicando que o emaranhamento entre o bombeio e prova se torna mais expressivo quando incluímos informações sobre o restante do sistema.

Entre o limiar e $\sigma=2,8$ observa-se que os autovalores simpléticos de todas as bipartições possíveis são menores que 1, esse tipo de emaranhamento é chamado de emaranhamento tripartite.

Lembrando que a parte simétrica da matriz de covariância difere da parte antissimétrica por uma rotação de $\pi / 2$, a análise tripartite da base antissimétrica fornece o mesmo resultado que a parte simétrica.

\subsubsection{Hexapartite}

Em nossa análise do sistema tripartite, não consideramos as correlações existentes entre a parte simétrica e antissimétrica do nosso sistema. Faremos essa análise considerando o sistema hexapartite formado polas partes simétrica e antissimétrica dos subsistemas de bombeio, prova e conjugado.

O resultado da análise do emaranhamento entre os subsistemas de bombeio, prova e conjugado, compostos por suas partes simétrica e antissimétrica, é apresentado na figura 3.20. Consideramos os autovalores simpléticos: $\nu_{s a 0}$ que caracteriza emaranhamento entre subsistema formado pelos modos simétrico e antissimétrico do bombeio e o restante do sistema, $\nu_{s a 1}=\nu_{s a 2}$, prova e o restante do sistema ou, conjugado e o restante do sistema, $\nu_{s a 01}$ bombeio e prova. O autovalor $\nu_{s a}$ caracteriza emaranhamento entre os modos simétricos dos campos de bombeio, prova e conjugado e os modos antissimétricos dos campos de bombeio, prova e conjugado. Devemos observar que as bipartições analisadas são do tipo: $2 \times 4,2 \times 2,3 \times 3$, e nesse caso a violação da condição de PPT é um critério suficiente para emaranhamento, mas não necessário. No intervalo analisado, $1<\sigma<4$, os subsistemas compostos pelos modos simétrico e antissimétrico dos campos de bombeio, prova e conjugado encontram-se emaranhados. 


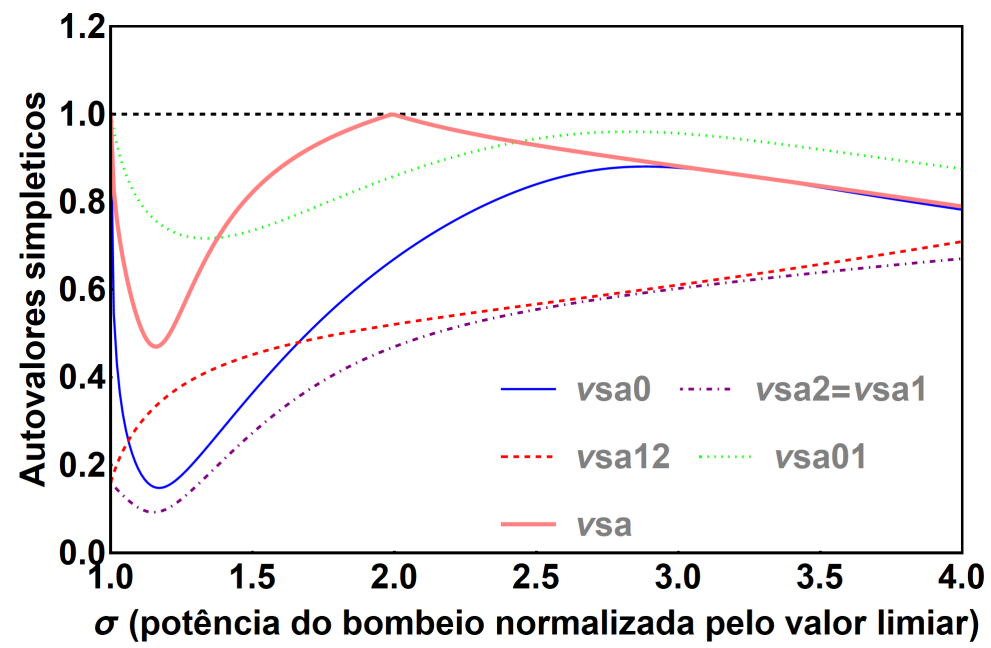

Figura 3.20: Teste de emaranhamento $\operatorname{PPT}(\Omega=0,5) . \nu(\mathrm{sa} n)$ - menor autovalor simplético obtido via transposição parcial de dois modos da matriz de covariância completa (parte simétrica e antissimétrica), onde os índices $\mathrm{n}=0,1,01,12$ representam os subsistemas referentes ao bombeio, prova, bombeio e prova, prova e conjugado respectivamente.

Além disso, observamos que existe emaranhamento entre as partes simétrica e antissimétrica do sistema completo (bombeio, prova e conjugado) em quase todo o intervalo analisado. A exceção é um ponto em torno de $\sigma=2$. Nesse ponto, o teste PPT é inconclusivo.

A figura 3.21 apresenta o os autovalores simpléticos encontrados para as bipartições 1 x 1 que medem o emaranhamento entre as partes simétrica e antissimétrica dos modos de bombeio, prova e conjugado. Os autovalores $\nu_{s 0 a 0}$, que caracteriza emaranhamento entre bombeio simétrico e antissimétrico, e $\nu_{s 1 a 1}$, prova simétrico e antissimétrico, são sempre maiores que 1 mostrando que não temos emaranhamento entre as partes simétrica e antissimétrica do bombeio e nem entre as partes simétrica e antissimétrica do prova. No caso dos autovalores $\nu_{s 0 a 1}$, que caracteriza emaranhamento entre bombeio simétrico e prova antissimétrico, e $\nu_{s 1 a 0}$, que caracteriza emaranhamento entre prova simétrico e bombeio antissimétrico, temos uma região de emaranhamento entre $2,5<\sigma<4$.

Aqui concluímos a análise das flutuações quânticas do DROPO com meio de ganho $\chi^{3}$. Vimos que o DROPO possui uma rica estrutura de emaranhamento nas proximidades do limiar que combina emaranhamento entre todas as possíveis diferentes bipartições do sistema e emaranhamento tripartite. 


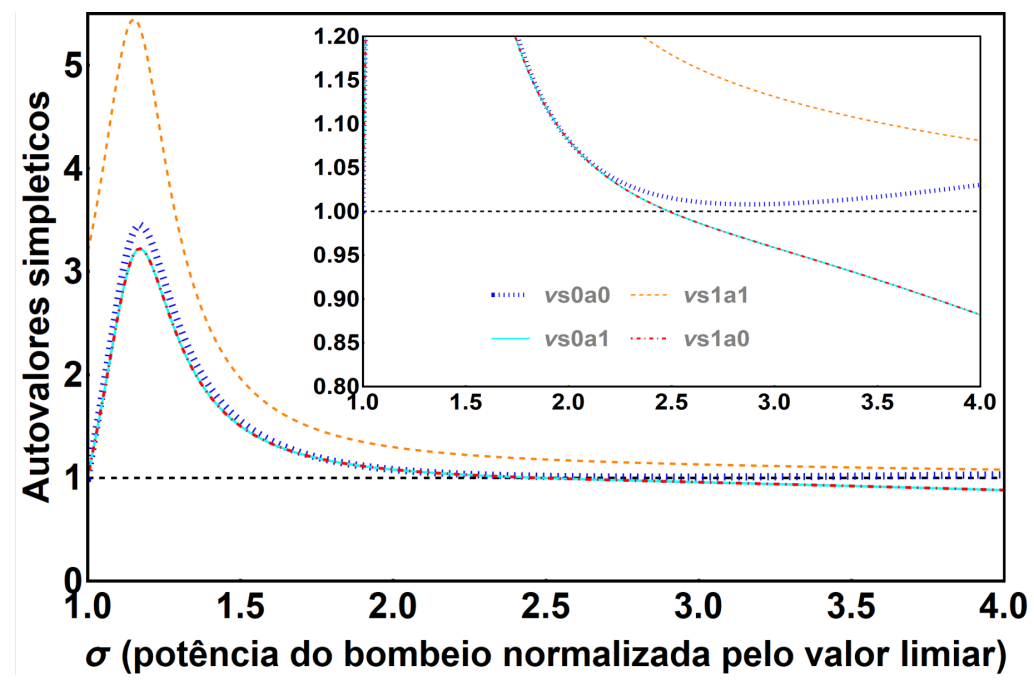

Figura 3.21: Teste de emaranhamento PPT $(\Omega=0,5)$. Autovalores simpléticos que representam emaranhamento entre: $\nu_{s 0 a 0}$ - bombeio simétrico e antissimétrico, $\nu_{s 1 a 1}$ - prova simétrico e antissimétrico, $\nu_{s 0 a 1}$ - bombeio simétrico e prova antissimétrico, $\nu_{s 1 a 0}$ - prova simétrico e bombeio antissimétrico.

\subsection{OPO triplamente ressonante na base simétrica e an- tissimétrica}

Esse trabalho conta com à análise das flutuações dos campos do sistema TROPO $\chi^{3}$ e comparações entre as características desse sistema e o sistema DROPO $\chi^{3}$. Assim como no caso do DROPO, o primeiro estudo que fazemos é o das flutuações das variâncias e correlações na base simétrica e antissimétrica.

$\mathrm{Na}$ figura 3.22 vemos o comportamento das variâncias de amplitude e fase dos campos de bombeio e prova na base simétrica. Vamos começar nossa análise pelo campo de bombeio do TROPO. Podemos ver que a quadratura amplitude apresenta um grande pico de excesso de ruído, bem mais expressivo do que o pico de excesso de ruído do DROPO, esse pico cai rapidamente mas não ultrapassa o ruído quântico padrão no intervalo analisado. Além disso, a quadratura de fase apresenta compressão de ruído em todo o intervalo analisado. No caso do DROPO, o comportamento do bombeio era parecido, no entanto, em torno de $\sigma=2,1$ as variâncias de amplitude e fase se cruzavam próximas de um ruído de 1,06 e a variância da quadratura amplitude se torna comprimida enquanto a variância da fase apresenta excesso de ruído. O comportamento das variâncias de amplitude e fase do campo de prova do TROPO é bem parecido com o DROPO. A variância do campo de bombeio apresenta um pico de excesso de ruído nas proximidades do limiar (sendo esse excesso de ruído muito maior que no caso do DROPO) que em $\sigma=1,87$ passa a apresentar compressão de ruído. A variância da quadratura fase apresenta excesso de ruído em todo o intervalo analisado, assim como no caso do DROPO.

As correlações não nulas da base simétrica do TROPO são mostradas na figura 3.23. Po- 


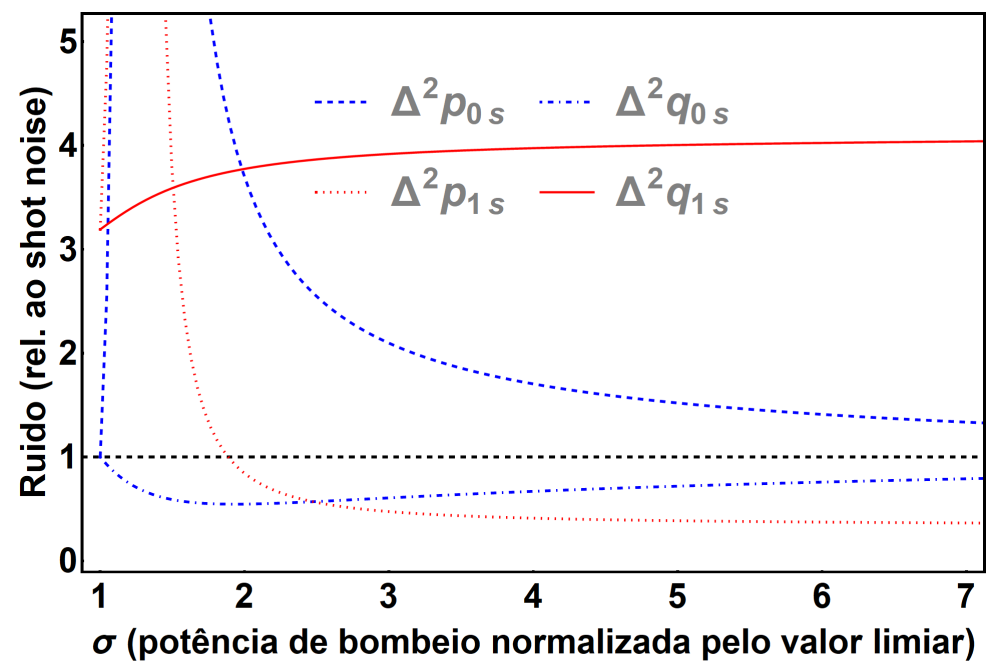

Figura 3.22: Variâncias da parte simétrica das quadraturas de amplitude e fase para os feixes de prova e conjugado em função da potência do bombeio $(\Omega=0,5) . \Delta^{2} p_{0 s}$ - variância da amplitude simétrica para o modo do bombeio; $\Delta^{2} p_{1 s}$ - variância da amplitude simétrica para o modo de prova; $\Delta^{2} q_{0 s}$ - variância da fase simétrica para o modo do bombeio; $\Delta^{2} q_{1 s}$ - variância da fase simétrica para o modo de prova.

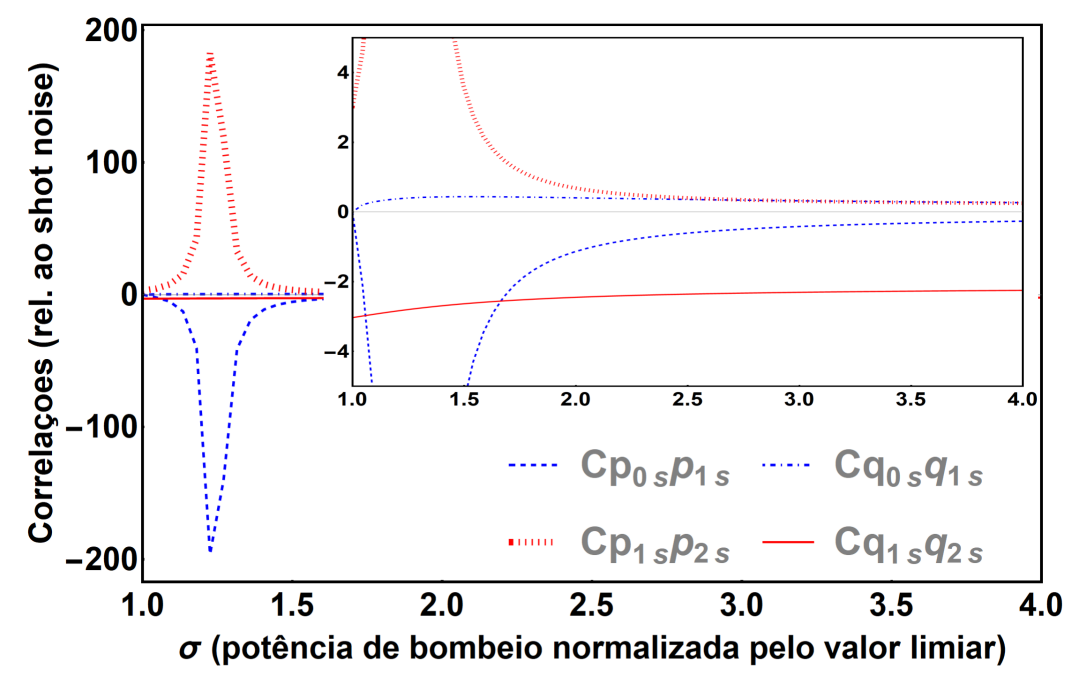

Figura 3.23: Parte simétrica das correlações entre bombeio, prova e conjugado $(\Omega=0,5): C p_{0 s} p_{1 s}$ - Correlação entre as quadraturas de amplitude simétricas do bombeio e prova; $C q_{0 s} q_{1 s}$ - Correlações entre as quadraturas de fase simétricas dos modos de bombeio e complementar; $C p_{1 s} p_{2 s}$ - Correlação entre as quadraturas de amplitude simétricas dos modos de prova e conjugado; $C q_{1 s} q_{2 s}$ - Correlações entre as quadraturas de fase simétricas dos modos de prova e conjugado.

demos ver que as quadraturas amplitudes dos campos de bombeio e prova apresentam grande anticorrelação enquanto as quadraturas fase desses mesmos dois campos apresentam uma pequena correlação. Quando analisamos as correlações entre os campos de prova e conjugado, ve- 
mos que a quadratura amplitude apresenta uma grande correlação, enquanto a quadratura fase apresenta uma pequena correlação. Esse comportamento é muito similar ao comportamento do DROPO abaixo da potência $\sigma=3$, contudo, as correlações entre as quadraturas amplitude do campo de bombeio e prova e amplitude dos campos prova e conjugado do DROPO apresentam valores muito menores.

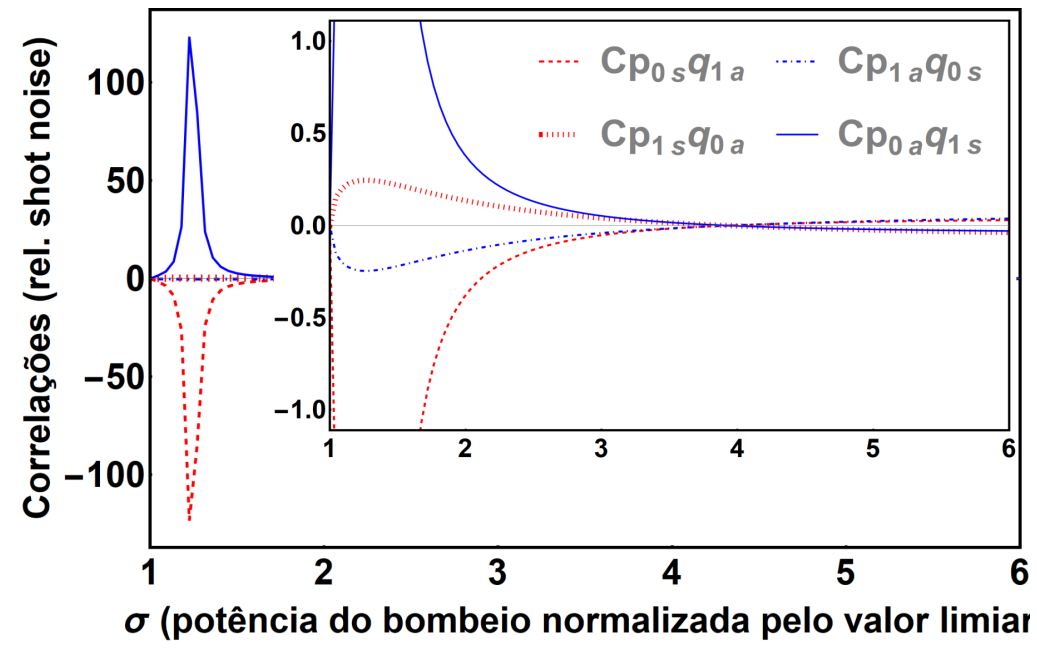

Figura 3.24: Correlações não nulas entre as quadraturas da base simétrica e antissimétrica em função da potência do bombeio $(\Omega=0,5)$. $C p_{0 s} q_{1 a}$ - Correlação entre a quadratura de amplitude simétrica do bombeio e a quadratura de fase antissimétrica do prova; $C p_{1 a} q_{0 s}$ - Correlação entre a quadratura de amplitude antissimétrica do conjugado e a quadratura de fase simétrica do bombeio; $C p_{1 s} q_{0 a}-$ Correlação entre a quadratura de amplitude simétrica do prova e a quadratura de fase antissimétrica do modo de bombeio; $C p_{0 a} q_{1 s}$ - Correlação entre a quadratura de amplitude antissimétrica do bombeio e a quadratura de fase simétrica do prova.

O principal foco desse trabalho é estudar as flutuações produzidas pelos campos do OPO $\chi^{3}$ entre $1 \leq \sigma \leq 4$ (bem próximo do limiar de oscilação), contudo, sempre que houver algo interessante para mostrar em uma região de potências um pouco mais altas faremos isso. A figura 3.24 apresenta as correlações não nulas entre as bases simétrica e antissimétrica para sigma entre $1 \leq \sigma \leq 6$. Vemos que existe uma grande correlação entre a quadratura amplitude simétrica do bombeio e a quadratura fase antissimétrica do conjugado $\left(C p_{0 s} q_{1 a}\right)$, enquanto um comportamento simétrico a esse é visto na anticorrelação entre a quadratura amplitude antissimétrica do campo de bombeio e a quadratura fase simétrica do prova $\left(C p_{0 a} q_{1 s}\right)$ até uma potência em torno de $\sigma=0$. Também vemos simetria entre a pequena correlação entre as quadraturas de amplitude simétrica do prova e a quadratura antissimétrica de fase do bombeio $\left(C p_{1 s} q_{0 a}\right)$ e a anticorrelação entre as quadraturas amplitude antissimétrica do prova e quadratura fase simétrica do bombeio $\left(C p_{1 a} q_{0 s}\right)$ até uma potência em torno de $\sigma=0$. Quando atingimos $\sigma \approx 4$ todas as correlações positivas passam a ser negativas e vice-versa, mas não mostram um crescimento expressivo se mantendo próximas de zero. Se compararmos esses 
resultados aos resultados obtidos na figura 3.13 para DROPO, percebemos que existe a mesma simetria, porém ao redor do ponto $\sigma=1,26$.

Finalmente, vamos fechar o estudo dos ruídos na base simétrica e antissimétrica analisando a informação fornecida pela pureza total e pela pureza dos campos individuais mostradas na figura 3.25 (a). Como era esperado a pureza do estado completo é igual à 1, o que serve como teste de sanidade. Vemos que tanto a pureza do bombeio como a pureza dos campos de prova e conjugado chega a praticamente zero em $\sigma=1,23$, isso se deve aos elevados ruídos de amplitude que todos os campos apresentam e as grandes correlações entre os campos ao redor do ponto $\sigma=1,23$. Porém a pureza não é nula pois isso só seria compatível com campos que apresentassem um excesso de ruído infinito em alguma de suas quadraturas, não é esse o caso em estudo. A pureza muito pequena mostra que devemos esperar um forte emaranhamento entre os três campos em estudo nessa região. Também percebemos que nos afastamos dessa região de grande excesso de ruído, $\sigma>3$, a pureza cresce, chegando a $\wp \approx 0,6$ para os campos de prova e conjugado e a $\wp \approx 0,9$ para o campo de bombeio. Comparando a pureza do TROPO com a pureza do DROPO vemos que a principal diferença entre os dois casos é que no caso do TROPO o excesso de ruído de amplitude dos campos envolvidos no 4WM é bem maior que no caso do DROPO e, consequentemente, a pureza cai a níveis mais baixos.

(a)

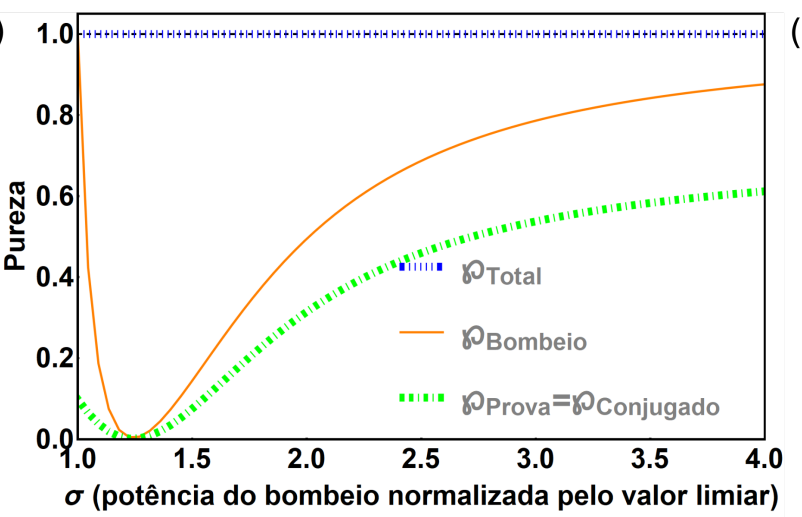

(b)

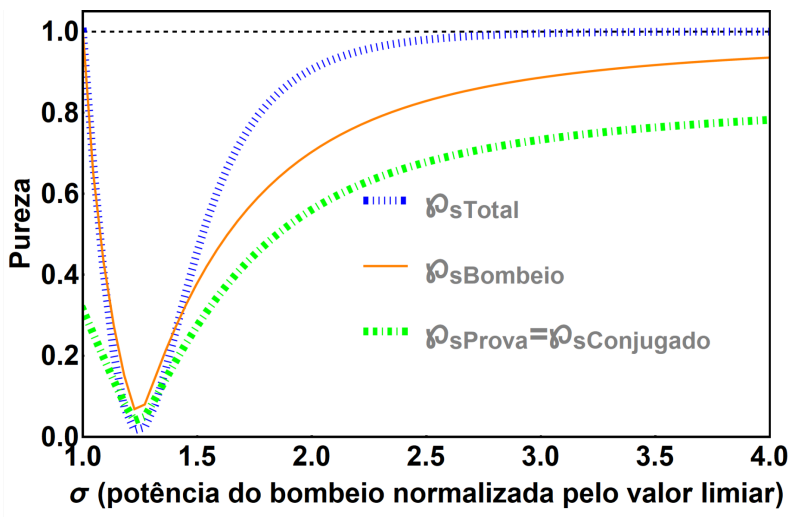

Figura 3.25: Pureza dos subsistemas formados pelas partes simétrica e antissimétrica do bom-

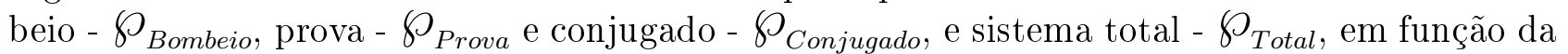
potência de bombeio normalizada para frequência de análise $(\Omega=0,5)$. (b) Pureza da parte

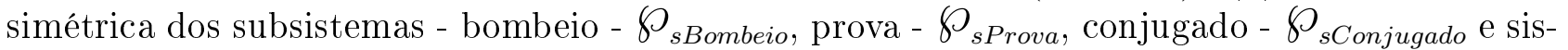
tema total simétrico - $\wp_{\text {sTotal }}$, em função da potência de bombeio normalizada para frequência de análise $(\Omega=0,5)$.

A figura 3.25 (b) apresenta os resultados da pureza da parte simétrica dos subsistemas de

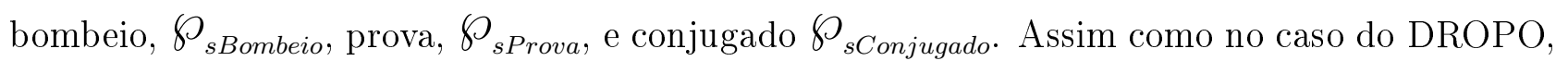
a pureza dos subsistemas que levam em conta apenas a parte simétrica da matriz de covariância é maior que a pureza dos subsistemas que levam em conta as partes simétrica e antissimétrica. Além disso, $\wp_{\text {stotal }}<1$ na região entre $1<\sigma<3$, mostrando que temo emaranhamento entre 
modos simétricos e antissimétricos dos campos. Notamos também que nas proximidades de $\sigma=4$, região em que temos a pureza da parte simétrica próxima a um, as correlações entre as partes simétrica e antissimétrica dos campos apresentadas na figura 3.24 se anulam.

\subsection{OPO triplamente ressonante na base soma e diferença}

Após análise das flutuações do campo na base simétrica e antissimétrica, estudamos as as flutuações na base da soma e da diferença. Lembrando que o teste de Duan para os campos de prova e conjugado é feito nessa base.

Na figura 3.26 (a), observamos que, pelos motivos já apresentados na discussão da figura 3.15 (a) do modelo do DROPO, as quadraturas amplitude e fase na base da diferença são independentes de potência de bombeio, sendo que a quadratura amplitude apresenta compressão de ruído e a quadratura fase apresenta excesso de ruído. No espaço da soma temos compressão de ruído da quadratura de soma das fases até a potência de $\sigma=1,57$, que é compensada por um grande pico de excesso de ruído na quadratura amplitude entre $1<\sigma<2$, 46. Para potências acima de $\sigma<2,46$ temos compressão de ruído na quadratura amplitude, enquanto a quadratura soma das fases apresenta excesso de ruído acima de $\sigma=1,57$. Observamos que no caso do DROPO $\Delta p_{+s}$ apresenta um excesso de ruído muito menor e que os pontos em que as quadraturas $\Delta p_{+s}$ e $\Delta q_{+s}$ cruzam o ruído quântico padrão ocorrem em potências menores, mas de forma geral o comportamento das variâncias do TROPO e DROPO é parecido.
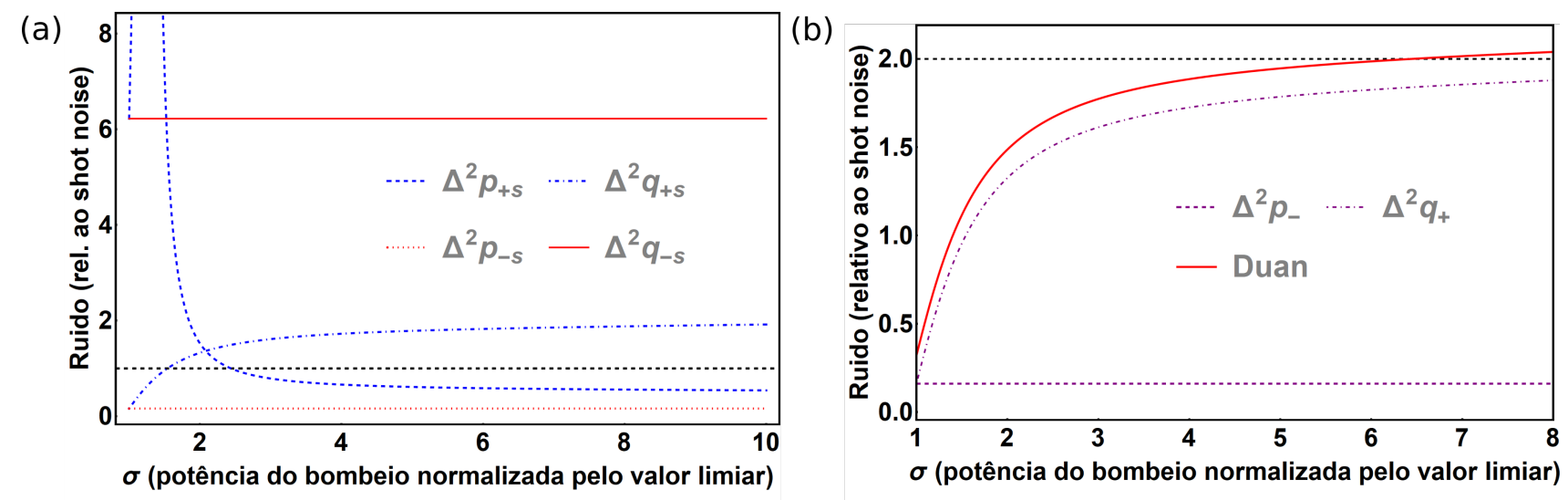

Figura 3.26: (a) Variâncias na base da soma e bombeio em função da potência do bombeio $(\Omega=0,5)$; (b) teste de Duan $(\Omega=0,5) . \Delta p_{-s}$ - Variância da amplitude simétrica do modo da diferença. $\Delta q_{-s}-$ Variância da fase simétrica do modo da diferença; $\Delta p_{+s}$ - Variância da amplitude simétrica do modo da soma; $\Delta^{2} q_{+s}$ - Variância da fase simétrica do modo da soma.

A figura 3.26 (b) apresenta o teste de Duan para o modelo do TROPO. Como podemos ver, os campos de prova e conjugado violam a desigualdade de Duan até uma potência de $\sigma=6$. Lembrando que no modelo do DROPO os campos de prova e conjugado deixavam de violar 
Duan em uma potência de $\sigma=2,26$, o TROPO é uma opção que oferece maior liberdade de escolha de potência para protocolos de teletransporte acima do limiar.

Na figura 3.27 (a) apresenta todas as correlações não nulas na base simétrica. Entre $1<$ $\sigma<2$ temos um grande pico de anticorrelação entre as quadraturas de amplitude da soma e do bombeio, para potências maiores que $\sigma=2$ essa anticorrelação se aproxima assintoticamente do ruído quântico padrão. Vemos também uma pequena correlação entre as quadraturas de fase da soma e do bombeio, essa pequena correlação se aproxima assintoticamente do ruído quântico padrão com o aumento da potência de bombeio. O comportamento das correlações simétricas na base simétrica para o TROPO é quase idêntico ao comportamento do DROPO, porém a anticorrelação de amplitude é muito maior.
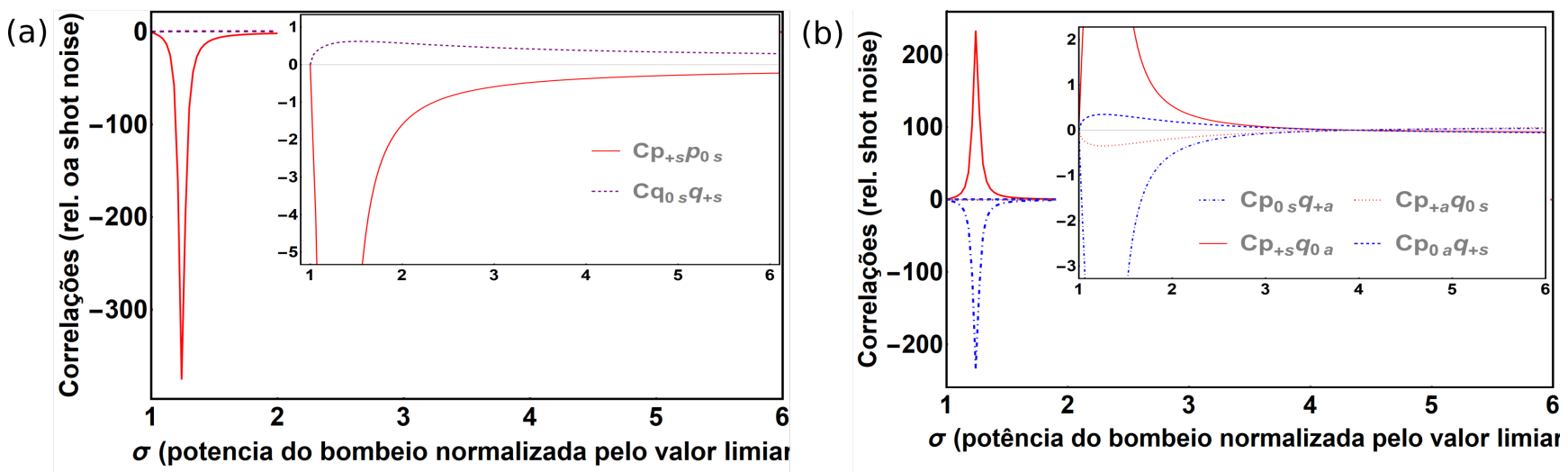

Figura 3.27: Parte (a): Correlações não nulas da parte simétrica das quadraturas no espaço soma e bombeio em função da potência de bombeio $(\Omega=0,5) . C p_{+s} p_{0 s}$ - Correlação entre parte simétrica da soma das amplitudes entre prova e conjugado e amplitude do bombeio. $C q_{0 s} q_{+s}$ - Correlação entre a parte simétrica do bombeio e parte simétrica da soma das fases entre prova e conjugado. Parte (b): Correlações não nulas entre as partes simétrica e antissimétrica das quadraturas em função da potência de bombeio $(\Omega=0,5)$. $C p_{0 s} q_{+a}$ - Correlação entre a quadratura antissimétrica de soma das fases com a quadratura simétrica de amplitude do bombeio; $C p_{+a} q_{0 s}$ - Correlação entre a quadratura antissimétrica da soma das amplitudes com a fase simétrica do bombeio; $C p_{+s} q_{0 a}$ - Correlação entre a quadratura soma das amplitudes com a fase do bombeio antissimétrico; $p_{0 a} C q_{+s}$ - Correlação entre a quadratura simétrica de soma das fases com a quadratura antissimétrica de amplitude do bombeio.

As correlações não nulas entre as bases simétrica e antissimétricas são apresentadas na figura 3.27 (b). Observando o comportamento das correlações no intervalo $1<\sigma<4$, vemos que existe um grande pico de correlação entre a quadratura de amplitude simétrica da soma e quadratura de fase antissimétrica do bombeio $\left(C p_{+s} q_{0 a}\right)$. Nessa mesma faixa de potência do bombeio, temos anticorrelação entre as quadraturas de amplitude simétrica do bombeio e quadratura de fase antissimetrica da soma $\left(C p_{0 s} q_{+a}\right)$ com comportamento oposto à $C p_{+s} q_{0 a}$. Também temos simetria entre a correlação entre as quadraturas amplitude simétrica do bombeio e fase antissimétrica da soma $\left(C p_{0 s} q_{+a}\right)$ e a anticorrelação entre as quadraturas de amplitude antissimétrica do bombeio e fase simétrica da soma $\left(p_{0 a} C q_{+s}\right)$. Em torno de $\sigma=4$ todas essas 
correlações cruzam o ruído quântico padrão e invertem seu sinal, o módulo dessas correlações se mantem bem próximo de zero. O modelo do DROPO apresenta simetrias similares as correlações entre as quadraturas na base simétrica e antisssimétrica.

$\mathrm{Na}$ figura 3.28(a) vemos o comportamento da pureza para os subsistemas formados pela parte simétrica e antissimétrica dos campos de bombeio $\left(\wp_{\text {Bombeio }}\right)$, soma $\left(\wp_{+}\right)$e diferença $\left(\wp_{-}\right)$entre prova e conjugado. Vemos que a pureza do subsistema da diferença entre os campos de prova é unitária. Já esperávamos este resultado, visto que o espaço do bombeio é desacoplado dos demais subespaços. Esse é um resultado trivial para OPOs. Sabemos que entre $1 \leq \sigma \leq 2$ temos grandes picos de variância da quadratura amplitude dos subespaços de prova e conjugado e também os picos de correlações que foram apresentados ao longo desse texto, o que justifica a grande queda na pureza do bombeio e da soma nessa região. Como tanto variâncias como correlações no TROPO apresentam valores muito maiores do que o que foi visto no caso do DROPO, a pureza cai a quase zero. Quando investigamos regiões de potência $\sigma>2$ observamos um crescimento monótono da pureza que chega a 0,9 em $\sigma=4$, esse comportamento se deve ao enfraquecimento das variâncias e correlações nessa região. Os resultados da pureza do TROPO apontam para a existência de emaranhamento tripartite, o qual investigaremos por meio do critério PPT na próxima seção. Além disso, pela análise da figura 3.28 (b) observamos que $\wp_{\text {stotal }}<1$ na região entre $1<\sigma<3$, caracterizando emaranhamento entre os modos simétrico e antissimétrico dos campos nesta região.

(a)

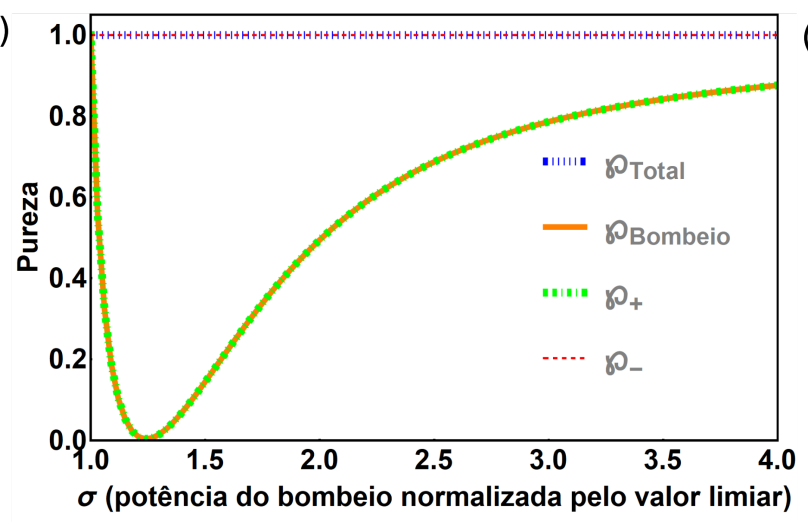

(b)

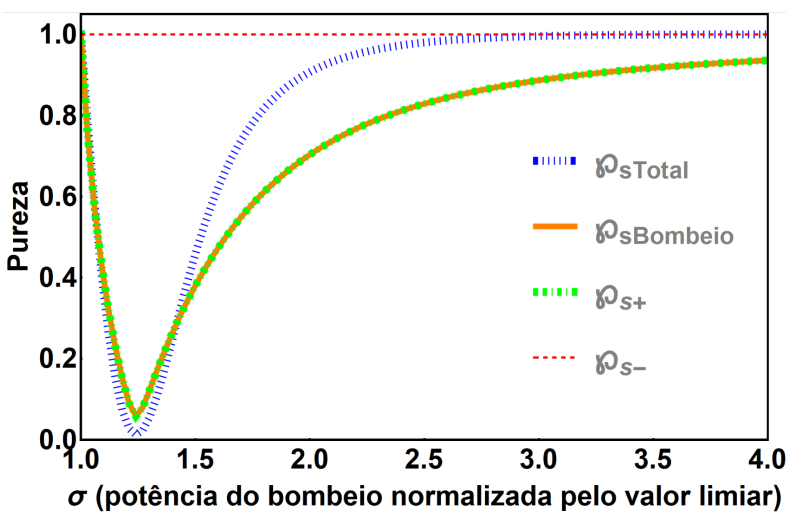

Figura 3.28: (a) Pureza dos subsistemas formados pelas partes simétrica e antissimétrica do bombeio - $\wp_{\text {Bombeio }}$, soma de prova e conjugado - $\wp_{+}$, diferença entre prova e conjugado - $\wp_{-}$, e sistema total - $\wp_{\text {Total }}$, em função da potência de bombeio normalizada para frequência de análise $(\Omega=0,5)$. (b) Pureza da parte simétrica dos modos do bombeio - $\wp_{\text {sBombeio }}$, soma de prova e conjugado - $\wp_{s+}$ e diferença entre prova e conjugado - $\wp_{s-}$, e sistema total simétrico $\wp_{\text {Total }}$, em função da potência de bombeio normalizada para frequência de análise $(\Omega=0,5)$. 


\subsection{Teste de positividade sob transposição parcial (TROPO)}

\subsubsection{Tripartite}

Nessa seção vamos analisar o emaranhamento entre diferentes bipartições do campos do TROPO por meio do critério PPT. Para essa análise utilizaremos as curvas apresentadas na figura 3.29. Essas curvas mostram os autovalores simpléticos que caracterizam o emaranhamento entre as bipartições: bombeio e o restante do sistema $(\nu 0)$, prova e o restante do sistema $(\nu 1)$, conjugado e o restante do sistema $(\nu 2)$, prova e conjugado $(\nu 12)$, bombeio e prova $(\nu 01)$.

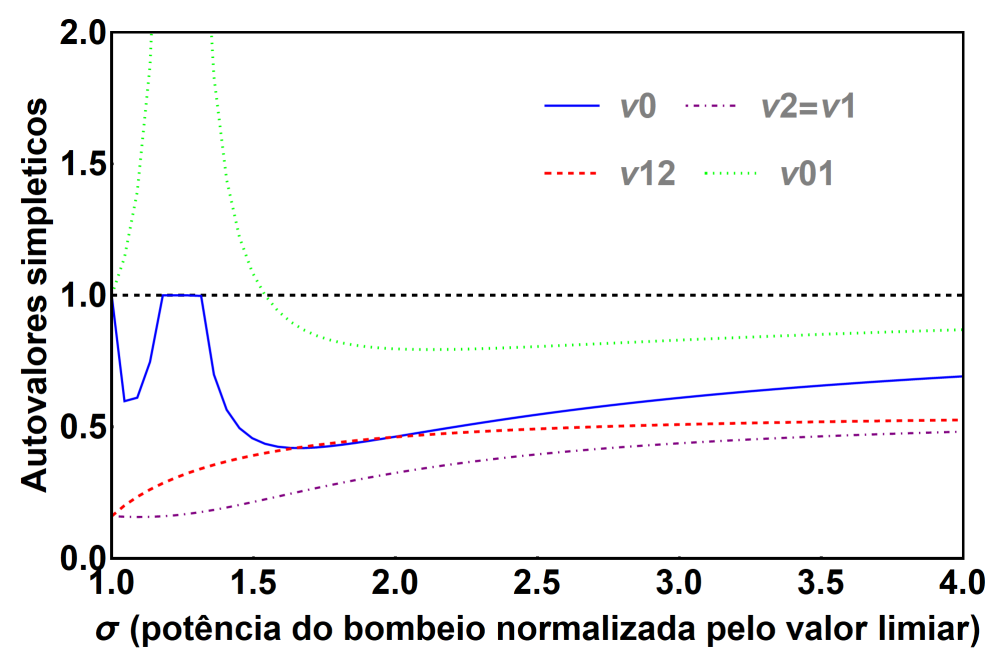

Figura 3.29: Teste de emaranhamento PPT $(\Omega=0,5) . \nu(\mathrm{n})$ - menor autovalor simplético obtido via transposição parcial do par de modos $\left(\omega_{n}+\Omega, \omega_{n}-\Omega\right)$ da matriz de covariância do OPO, onde os índices $\mathrm{n}=0,1,01,12$ representam os subsistemas referentes ao bombeio, prova, bombeio e prova, prova e conjugado respectivamente.

Pela análise gráfica vê-se que $\nu 0$ é menor que 1 em quase toda a região analisada $(1<\sigma<4)$. No entanto, entre $1,15<\sigma<1,31$ temos uma quebra de emaranhamento. Os autovalores $\nu 1$ e $\nu 2$ são iguais e pela análise gráfica vemos que as bipartições representadas por esses dois autovalores estão emaranhadas com o restante do sistema entre $1 \leq \sigma \leq 4$.

Pela análise do autovalor $\nu 12$ vemos que os campos de prova e conjugado encontram-se emaranhados entre $1 \leq \sigma \leq 4$. Vemos também que $\nu 12$ é maior que $\nu 1$ em toda a região analisada, indicando que o emaranhamento entre prova e conjugado se torna mais expressivo quando incluímos informações sobre o bombeio.

Pela análise de $\nu 01$ vemos que bombeio e prova (ou bombeio e conjugado) estão emaranhados entre $1,53 \leq \sigma \leq 4$. Podemos notar $\nu 01$ é menor que $\nu 0$, e menor que $\nu 1$ o que indica que o emaranhamento entre o bombeio e prova se torna mais evidente quando incluímos informações sobre o restante do sistema. 
Entre 1,53 $\leq \sigma \leq 4$ observa-se que os autovalores simpléticos de todas as bipartições possíveis são menores que 1, portanto temos emaranhamento tripartite nessa região.

Também é interessante comparar os resultados dos autovalores simpléticos encontrados para o DROPO aos valores encontrados para o TROPO. Comparando a região $1 \leq \sigma \leq 2$ vemos que autovalores $\nu 1$ e $\nu 12$ tem um comportamento muito parecido nos dois sistemas. Por outro lado, os autovalores $\nu 0$ apresentam comportamentos bem diferentes nessa mesma região. No caso do TROPO temos $\nu 0$ perdendo emaranhamento nas proximidades do limiar, enquanto no caso do DROPO $\nu 0$ atinge seu mínimo nessa região. Algo parecido acontece com $\nu 01$ que no caso do TROPO está desemaranhado na região do limiar e no caso do DROPO atinge seu mínimo nessa região. Quando olhamos a região entre $2<\sigma \leq 4$ do TROPO, vemos que todos os autovalores simpléticos apresentam um leve crescimento, mas não chegam a um. No caso do DROPO, o crescimento é mais expressivo, sendo que $\nu 0$ e $\nu 01$ apresentam regiões de desemaranhamento nessa região.

\subsubsection{Hexapartite}

A análise do sistema hexapartite é feita considerando a contribuição que os subsistemas da parte simétrica e antissimétrica da matriz de covariância dão ao emaranhamento. Na figura 3.30 apresentamos o resultado do teste PPT para os autovalores $\nu_{s a 0}, \nu_{s a 1}, \nu_{s a 01}, \nu_{s a 12}$. Em todos os caso obtivemos autovalores menores que 1 , assim como ocorreu no caso do DROPO. Mostrando que os subsistemas representados por esse autovalores apresentam emaranhamento. Ainda nessa figura, vemos o autovalor $\nu_{s a}$ que caracteriza emaranhamento entre os subsistemas formados pelas partes simétrica e antissimétrica dos campos de bombeio, prova e conjugado combinados. Esse sistema apresenta uma pequena região de desemaranhamento ao redor de $\sigma=4$.

Os últimos autovalores simpléticos de interesse são $\nu_{s 0 a 0}, \nu_{s 1 a 1}, \nu_{s 0 a 1}, \nu_{s 1 a 0}$, em todos os casos, a análise apresentada na figura 3.31 mostra resultado maior que 1. Dessa forma, os subsistemas representados por esses autovalores não apresentam emaranhamento. 


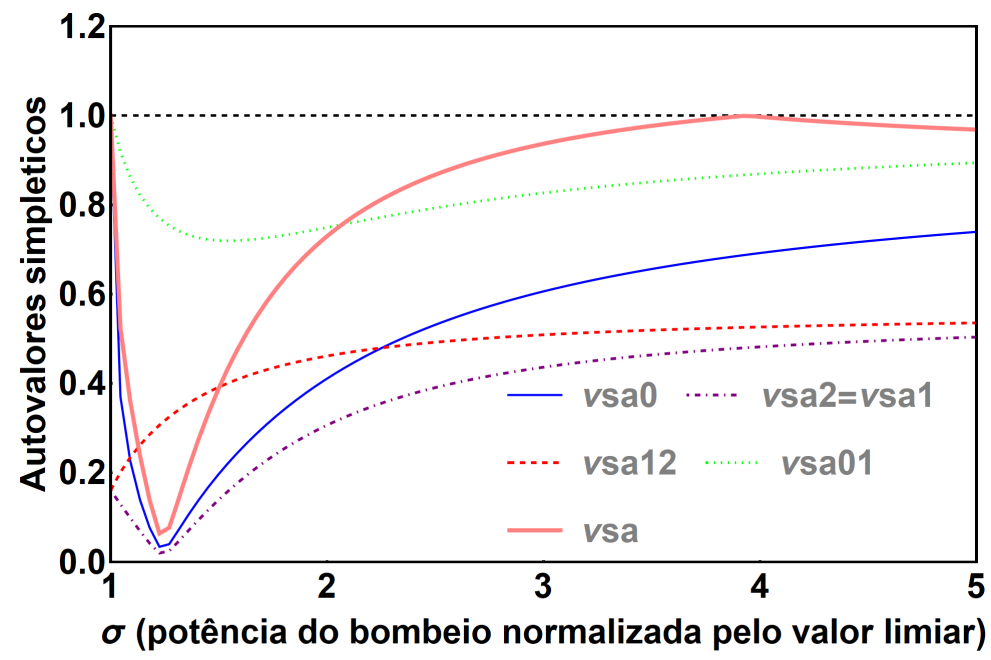

Figura 3.30: Teste de emaranhamento $\operatorname{PPT}(\Omega=0,5) . \nu(\mathrm{sa} n)$ - menor autovalor simplético obtido via transposição parcial de dois modos da matriz de covariância completa (parte simétrica e antissimétrica), onde os índices $\mathrm{n}=0,1,01,12$ representam os subsistemas referentes ao bombeio, prova, bombeio e prova, prova e conjugado respectivamente.

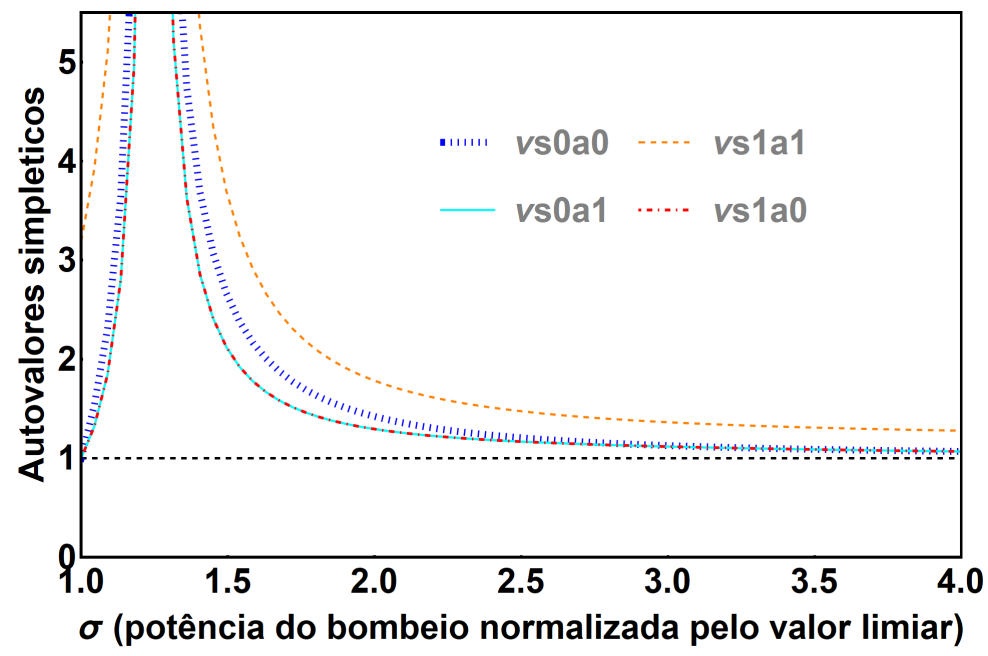

Figura 3.31: Teste de emaranhamento PPT $(\Omega=0,5)$. Autovalores simpléticos que representam emaranhamento entre: $\nu_{s 0 a 0}$ - bombeio simétrico e antissimétrico, $\nu_{s 1 a 1}$ - prova simétrico e antissimétrico, $\nu_{s 0 a 1}$ - bombeio simétrico e prova antissimétrico, $\nu_{s 1 a 0}$ - prova simétrico e bombeio antissimétrico.

Depois dessa extensa discussão dos modelos teórico do DROPO e do TROPO, vale a pena destacar os principais resultados desse capítulo:

- Modelamos a amplitude dos campos médios do bombeio, prova e conjugado para DROPO e TROPO com meio de ganho $\chi^{3}$, sendo esses modelos resultados inéditos.

- Inserimos a amplitude dos campos médios dos modos de bombeio, prova e conjugado no 
modelo desenvolvido em [53] e reconstruímos a matriz de covariância do sistema. Por meio dessa matriz de covariância, estudamos as flutuações quânticas do sistema considerando uma aproximação de estados Gaussianos.

- O hamiltoniano do 4WM utilizado nesse trabalho leva em conta a depleção do bombeio, fenômeno importante no estudo com cavidades. Nosso estudo difere dos estudos mais recentes à respeito do fenômeno de mistura de quatro ondas, pois esses estudos consideram o 4WM ocorrendo em espaço livre e dessa forma, a depleção do campo de bombeio é desprezível e esse campo é considerado como um campo clássico.

- Destacamos que o sistema DROPO apresenta compressão de ruído da quadratura amplitude do bombeio, que se trata de um resultado inédito. Além disso, os dois sistemas apresentam regiões em que ocorre compressão de ruído de amplitude dos campos convertidos e compressão de de ruído da fase desses campos.

- Fizemos uma caracterização bastante completa das regiões de emaranhamento dos sistemas DROPO e TROPO $\chi^{3}$. Utilizamos o teste de Duan para determinar regiões em que os campos de prova e conjugado podem ser usados para aplicação em protocolos de informação quântica. Por meio do teste PPT fizemos a caracterização do sistema tripartite formado pelos modos simétricos dos campos de bombeio, prova e conjugado e em seguida a caracterização das regiões de emaranhamento de um sistema hexapartite formado pelos modos simétrico e antissimétrico dos campos de bombeio, prova e conjugado. 


\section{Capítulo 4}

\section{Experimento}

Nesse capítulo faremos a descrição da montagem experimental idealizada com a qual seria possível fazer uma análise mais completa dos campos emitidos pelo OPO $\chi^{3}$ e as diferenças em relação ao experimento efetivamente implementado. Iremos apresentar os principais parâmetros que caracterizam o OPO em estudo e em seguida apresentar o trabalho que foi feito para caracterizar as flutuações de dois dos três diferentes tipos de campos produzidos por esse experimento. Os resultados apresentados nesse capítulo foram obtidos com a colaboração do doutor Hans Marin Florez que atualmente ocupa o cargo de pós-doutorando do grupo e dos trabalhos de mestrado de Harold Alberto Rojas Páez que caracterizou o fenômeno de 4WM em célula de rubídio em espaço livre [56], e Alvaro Montaña Guerrero (atualmente doutorando do grupo) que caracterizou os campos médios emitidos pelo OPO $\chi^{3}$ [57]. Nesse trabalho, fazemos a caracterização das flutuações desses campos emitidos pelo OPO $\chi^{3}$.

\subsection{Implementação do experimento}

O arranjo mostrado esquematicamente na figura 4.1 é a versão idealizada do experimento com o qual trabalhamos. Nessa figura podemos ver dois espelhos, $R_{1}$ e $R_{2}$, de reflectividades de $100 \%$ formando uma cavidade ótica. No interior dessa cavidade temos dois divisores de feixes por polarização (polarized beam splitter - PBS), uma lâmina de $\lambda / 4$ e uma célula de rubídio. Para facilitar a descrição desse experimento, denominamos os PBSs no interior do OPO por PBS 1 e PBS 2. Fazemos com que um feixe de bombeio intenso de polarização vertical e um feixe de prova de polarização horizontal se sobreponham e atravessem o PBS 2. O feixe de bombeio atravessa a célula de rubídio, atinge o espelho de $R_{2}=100 \%$ e é refletido de volta pelo mesmo caminho da ida. Devido à sua polarização horizontal, o feixe de prova atravessará o PBS 2 e sairá da cavidade sendo direcionado a um circuito de detecção homodina para servir de oscilador local. No entanto, o PBS não é perfeito, possuindo um erro de 1\%, ou seja, $1 \%$ do campo incidente de polarização horizontal sofre reflexão, como o campo de prova possui 
polarização horizontal, passará a cavitar dentro do OPO e servirá como semente para o 4WM.

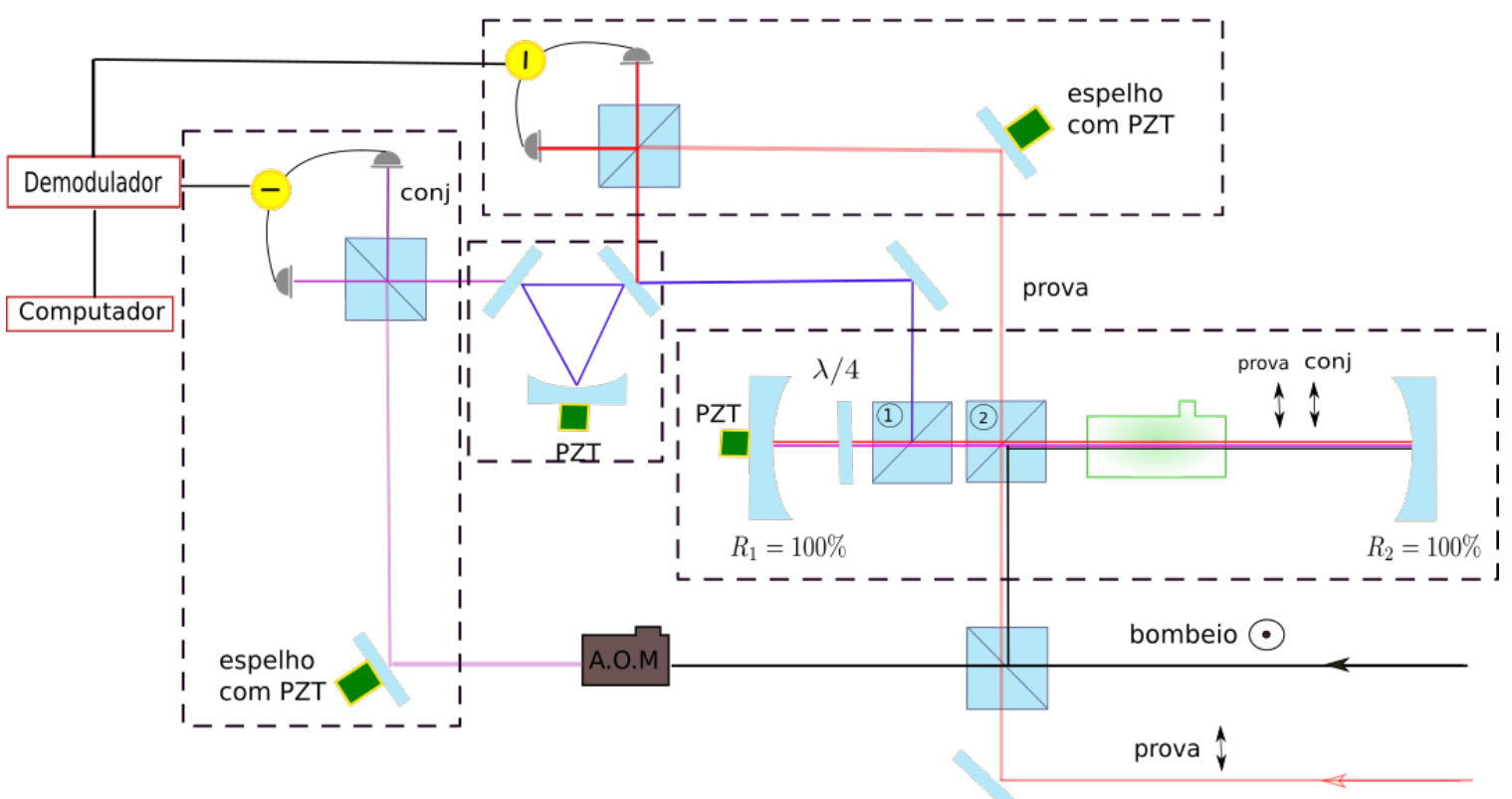

Figura 4.1: Esquema do experimento: Mostra os feixes de bombeio e de prova gerando 4WM dentro de uma cavidade. Os feixes do 4WM seguem para uma cavidade de separação de feixes e logo em seguida são detectados pelo método da detecção homodina.

Quando a semente do feixe de prova e o feixe de bombeio atravessam a célula de rubídio ocorre o fenômeno de $4 \mathrm{WM}$, o feixe de prova é intensificado e o feixe conjugado é criado, ambos com polarização horizontal. Prova e conjugado são refletidos pelo espelho $R_{2}$, fazem o percurso dentro da cavidade até o espelho $R_{1}$ e são refletidos de volta até atingir o PBS 1 . Observa-se que prova e conjugado fazem duas passagens pela lâmina de $\lambda / 4$, e essa muda a polarização desses feixes que passa a ter uma componente vertical e uma componente horizontal. A componente horizontal dos feixes continuará cavitando, enquanto a componente vertical será extraída da cavidade pelo PBS 1. A porção do campo que sai da cavidade depende do ângulo entre a direção de polarização dos feixes em relação ao eixo da lâmina de $\lambda / 4$. Assim, mudando esse ângulo muda-se a transmissão da cavidade. Quando a transmissão aumenta a cavidade se torna mais aberta e quando ela diminui a cavidade se torna mais fechada.

Os feixes de prova e conjugado deixam a cavidade sobrepostos, portanto é necessário separalos para fazermos a análise dos dados. Utilizamos a cavidade de separação de feixes apresentada na figura para esse fim. Tal cavidade é ressonante com o feixe conjugado, sendo assim, ele é transmitido pela cavidade e o feixe de prova é refletido.

A primeira diferença entre o experimento que efetivamente montamos para o experimento idealizado é que não dispúnhamos de um campo coerente para gerar a semente do campo 
de prova. Deixamos o campo que gera a semente do feixe de prova e oscilador local quase transparentes na figura 4.1 para destacar essa diferença. Isso não é um problema que nos impeça de gerar 4WM no OPO, pois a cavidade ótica amplifica o campo do vácuo de forma que um de seus modos amplificados servirá como semente do campo de prova. O maior problema é que sem o campo coerente que gera a semente não temos o oscilador local necessário para a homodinagem do campo de prova.

A segunda diferença é que não chegamos a dessintonizar o campo de bombeio para gerar um oscilador local na frequência do campo conjugado. Destacamos essa diferença na figura 4.1 deixando o campo do oscilador local do feixe conjugado quase transparente. No entanto, é possível iniciar a caracterização do sistema sem gerar a dessintonia. Em parte porque desejamos estudar 4WM degenerado e, nesse caso, prova e conjugado são produzidos na mesma frequência que o campo de bombeio. Em parte porque no caso do 4WM não degenerado, em uma etapa preliminar a detecção homodina, podemos fazer a detecção balanceada e medir compressão de ruído da quadratura diferença de amplitude entre prova e conjugado que já é um resultado muito interessante.

\subsection{Características do laser Ti:safira}

O feixe de bombeio desse experimento é produzido por um laser Ti:safira que foi desenvolvido no laboratório, esse laser é bombeado por um Verdi-V10 da Coherent que opera entre $730 \mathrm{~nm}$ e $800 \mathrm{~nm}$, sendo sua frequência ajustável por um etalon. O laser é CW (continuous wave) possui uma largura de linha com limite superior de $8,5 \mathrm{MHz}$ [58]. Esse laser é levado à mesa do experimento por meio de uma fibra ótica (veja o apêndice D). Atualmente esse acoplamento fornece $150 \mathrm{~mW}$ ao experimento.

O Ti:safira está dessintonizado $800 \mathrm{MHz}$ acima da transição $5 S_{1 / 2}, \mathrm{~F}=2 \rightarrow 5 P_{1 / 2}$ da linha D1 do rubídio. Mais especificamente o comprimento de onda em que sintonizamos o Ti:safira é de $794,9741 \mathrm{~nm}$.

A luz desse laser é coerente e na figura 4.2 apresentamos uma medida de do ruído de intensidade desse laser obtido por meio da detecção balanceada. Como podemos observar, a intensidade do ruído da quadratura amplitude do Ti:safira coincide com o ruído quântico padrão e trabalhos anteriores mostram que o ruído de fase também é limitado ao ruído quântico padrão $[58,59]$. 


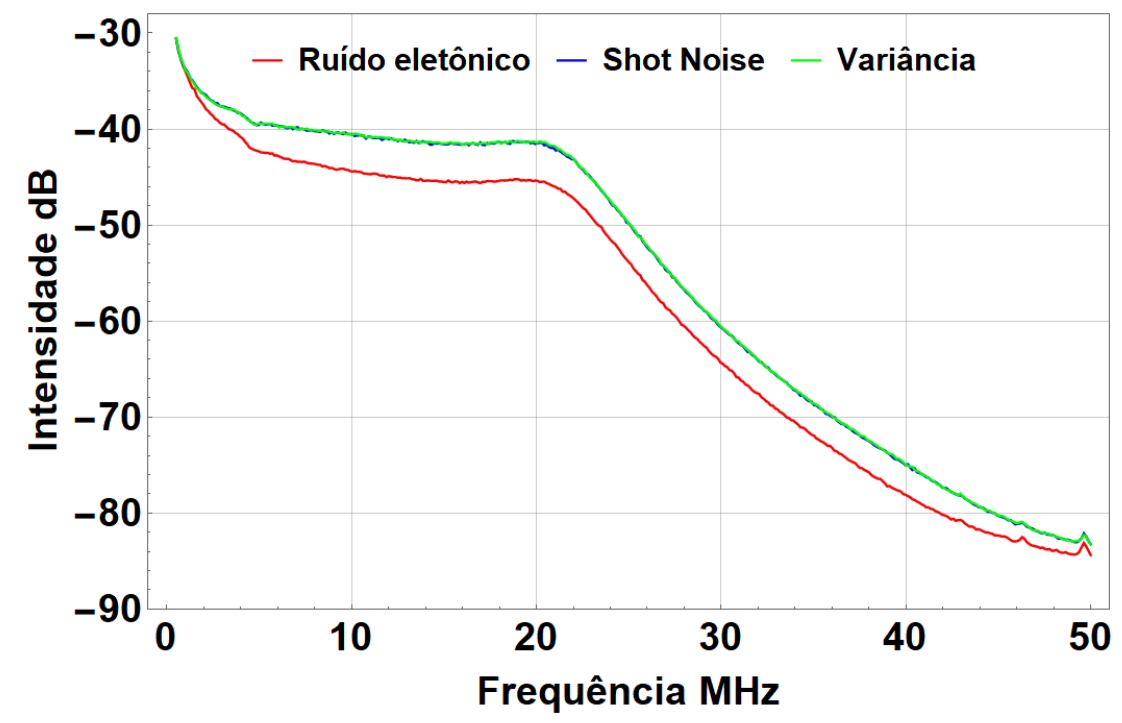

Figura 4.2: Ruído de amplitude do Ti:safira: Em vermelho vemos o ruído eletrônico do detector, em azul o ruído quântico padrão e em verde a variância da quadratura amplitude da luz fornecida pelo Ti:safira.

\subsection{Características da célula de rubídio}

A célula de vapor de rubídio utilizada no experimento foi produzida na USP de São Carlos com janelas de entrada inclinadas de modo a formar um ângulo de Brewster, $\theta_{B}$, com o feixe do bombeio. A inclinação do ângulo de Brewster garante que a janela da célula transmita toda a toda a luz que chega horizontalmente polarizada em relação a seu plano de incidência (polarização P). Inicialmente a luz do feixe de bombeio se propaga no ar que possui índice de refração $n_{1}=1$, então atinge a parede de vidro da célula de rubídio cujo índice de refração é de $n_{2}=1,5$. Para que o ângulo de incidência, $\theta_{1}$, seja o ângulo de Brewster, ele deve somar $90^{0}$ com o ângulo do feixe refratado, $\theta_{2}$. Substituindo essa condição na equação de Snell, temos:

$$
\theta_{B}=\arctan \left(\frac{n_{2}}{n_{1}}\right)
$$

que para a célula de rubídio é de, aproximadamente, $\theta_{B}=56,31^{0}$.

Sabendo que os campos de prova e conjugado incidem na célula de rubídio na polarização $\mathrm{P}$, essa configuração garante minimização das perdas desses campos nas janelas da célula.

\subsection{Características do OPO}

Como mencionado anteriormente, o OPO em estudo nesse projeto é formado por um laser de bombeio que incide em uma cavidade ótica que contém um meio de ganho de susceptibilidade não linear de terceira ordem em seu interior, assim como é visto na figura 4.3. 


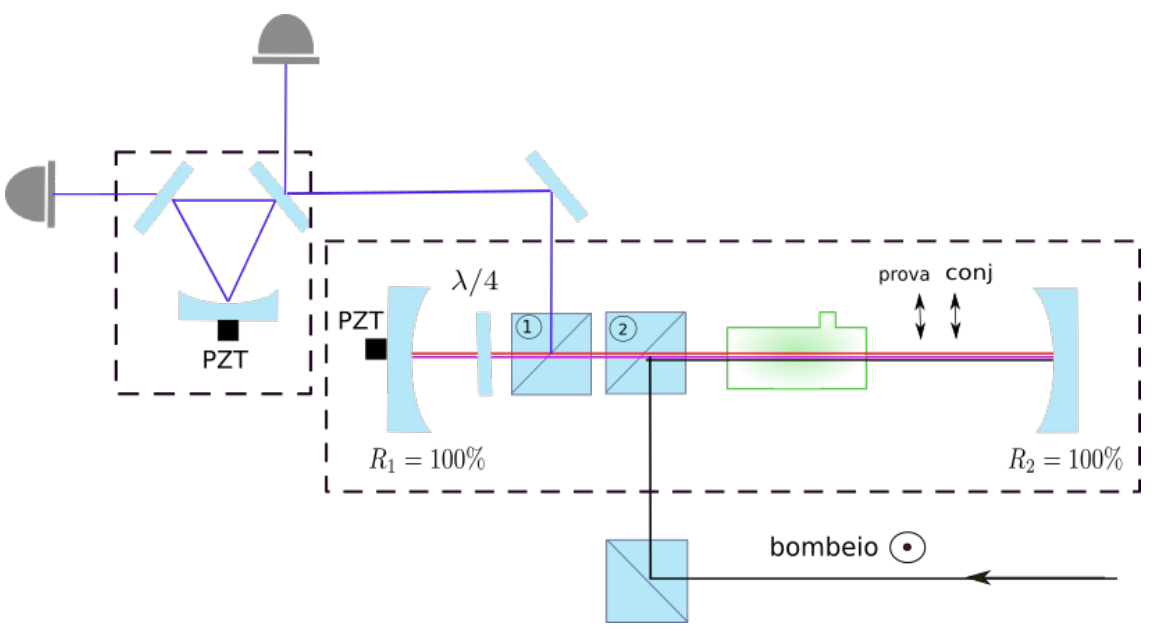

Figura 4.3: OPO duplamente ressonante: $O$ feixe de prova de polarização vertical entra na cavidade pelo PBS (2), atravessa o meio de ganho não linear de terceira ordem e gera dois outros feixes, prova e conjugado. Após ser refletido por um espelho de $100 \%$ de refletividade, ele sai da cavidade voltando pela mesma trajetória de entrada. A saída dos feixes gerados é feita por meio do PBS (1) enquanto a finesse da cavidade é controlada por meio da placa de $\lambda / 4$ inserida no interior da cavidade. Após a cavidade do OPO temos uma cavidade em anel com detectores para transmissão e reflexão.

A cavidade ótica do OPO possui uma finesse variável controlada pelo mecanismo de entrada e saída dos campos na cavidade apresentado na figura 4.1. Tal cavidade é formada por dois espelhos HR de raio de curvatura $R=500 \mathrm{~mm}$ da empresa ATFilms (v2-2364 \& v2-2367) (apêndice A), dois divisores de feixes por polarização (polarizing beam splitter, PBSs) da empresa Thorlabs de uma polegada e revestimento específico para a região em torno de $780 \mathrm{~nm}$ e uma placa de quarto de onda, $\lambda / 4$, Thorlabs de ordem zero específica para trabalhar na faixa de comprimento de onda ao redor de $780 \mathrm{~nm}$.

Lembrando que desejamos um OPO duplamente ressonante, é interessante construir uma cavidade que quando ressonante com os modos de prova e conjugado possa filtrar o bombeio. Sabendo que no caso do 4WM não degenerado os campos de prova e conjugado, estão dessintonizados $3 \mathrm{GHz}$ da frequência do bombeio, abaixo e acima, respectivamente. Construímos uma cavidade tal que $6 \mathrm{GHz}$ represente um múltiplo ímpar do intervalo espectral livre (free spectral range, FSR) e dessa forma exclua o bombeio quando em ressonância com os modos de prova e conjugado. Sendo assim, escolhemos trabalhar com uma cavidade de $666 \mathrm{MHz}$ de FSR.

Por meio da placa de quarto de onda que se encontra no interior do OPO podemos variar a finesse da cavidade dentro da extensão de $1,7<\mathrm{F}<37,8$, o que corresponde a variar a largura de banda entre 0,392 $\mathrm{GHz}>\Delta \omega_{O P O}>17,6 \mathrm{GHz}$. Na figura Figura 4.4 apresentamos os resultados da caracterização da finesse em função do ângulo da placa de quarto de onda.

Na tabela 4.4 destacamos as principais características do OPO que foram apresentadas: 


\begin{tabular}{|l|l|}
\hline Características do OPO & \\
\hline FSR & $666 \mathrm{MHz}$ \\
\hline Finesse & $1,7 \leq \mathrm{F} \leq 37,8$ \\
\hline Largura de banda & $0,392 \mathrm{GHz} \geq \Delta \omega_{O P O} \geq 17,6 \mathrm{MHz}$ \\
\hline Temperatura da célula & $60^{\circ} \mathrm{C} \leq \mathrm{T} \leq 86^{\circ} \mathrm{C}$ \\
\hline
\end{tabular}

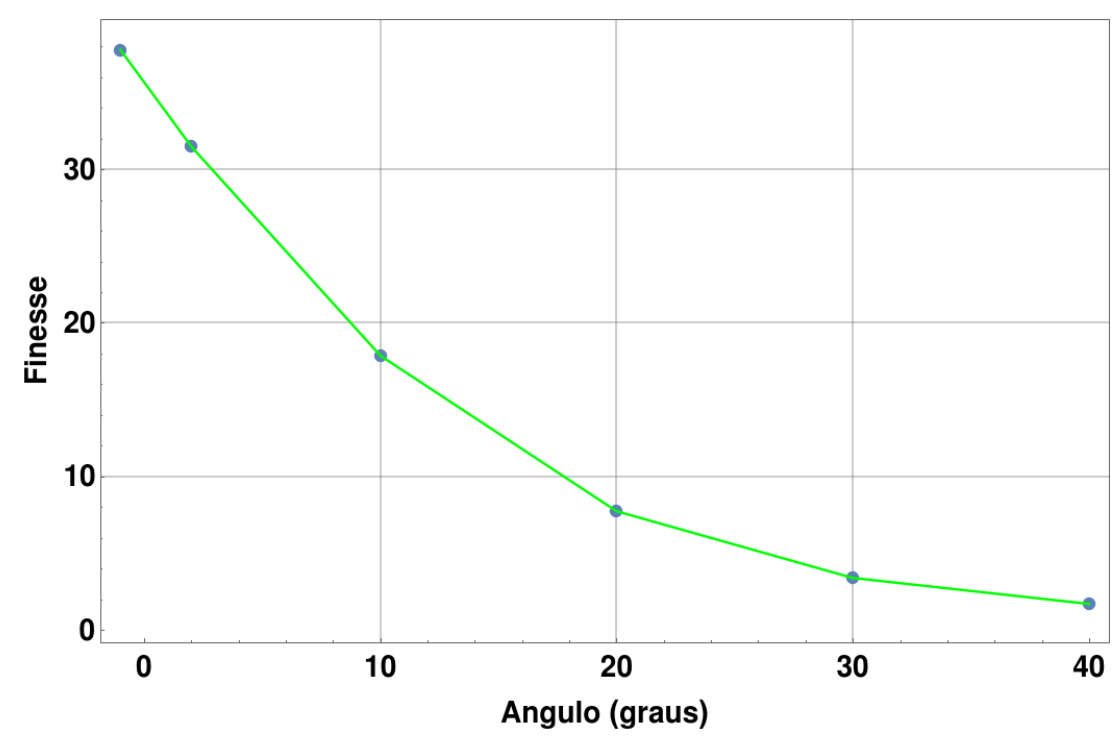

Figura 4.4: Finesse em função do ângulo da placa de quarto de onda.

\subsubsection{Campos gerados para célula de rubídio à $86^{\circ} \mathrm{C}$}

Passamos ao estudo dos feixes gerados pelo OPO. Na figura 4.5 vemos os picos característicos do nosso OPO quando opera a uma temperatura de $86^{\circ} \mathrm{C}$, escolhemos essa temperatura devido aos autos ganhos do 4WM nessa temperatura [57, 56]. Nessa condição, observamos que o limiar de oscilação do OPO é cruzado duas vezes a medida que aumentamos a potência do bombeio. Na parte (a) da figura 4.5 vemos dois tipos diferentes de picos, o $1^{\circ}$ pico é gerado quando o bombeio atinge uma potência maior que $5,8 \mathrm{~mW}$, enquanto o $2^{\circ}$ pico é gerado quando o bombeio atinge uma potência maior que $13,6 \mathrm{~mW}$. A parte parte (b) da figura 4.5 mostra o sinal captado por detectores colocados após a cavidade em anel mostrada na figura 4.3. Os picos verdes são o resultado obtido na situação em que travamos o OPO no $1^{\circ}$ pico e em laranja o resultado obtido na situação em que travamos o OPO no $2^{\circ}$ pico. Observamos que a distância entre os picos consecutivos em laranja corresponde a aproximadamente metade da distância entre os picos em verde. Inicialmente esse resultado nos fez acreditar que o $1^{\circ}$ pico era resultado de $4 \mathrm{WM}$ degenerado (campos de prova e conjugado sendo criados com a mesma potência do bombeio) e que o $2^{\circ}$ pico era resultado de $4 \mathrm{WM}$ não degenerado, (campos de prova e conjugado sendo 
criados dessintonizados do bombeio). No entanto, não conseguimos fazer a interferência entre o campo do $1^{\circ}$ pico e o campo do laser Ti:safira.
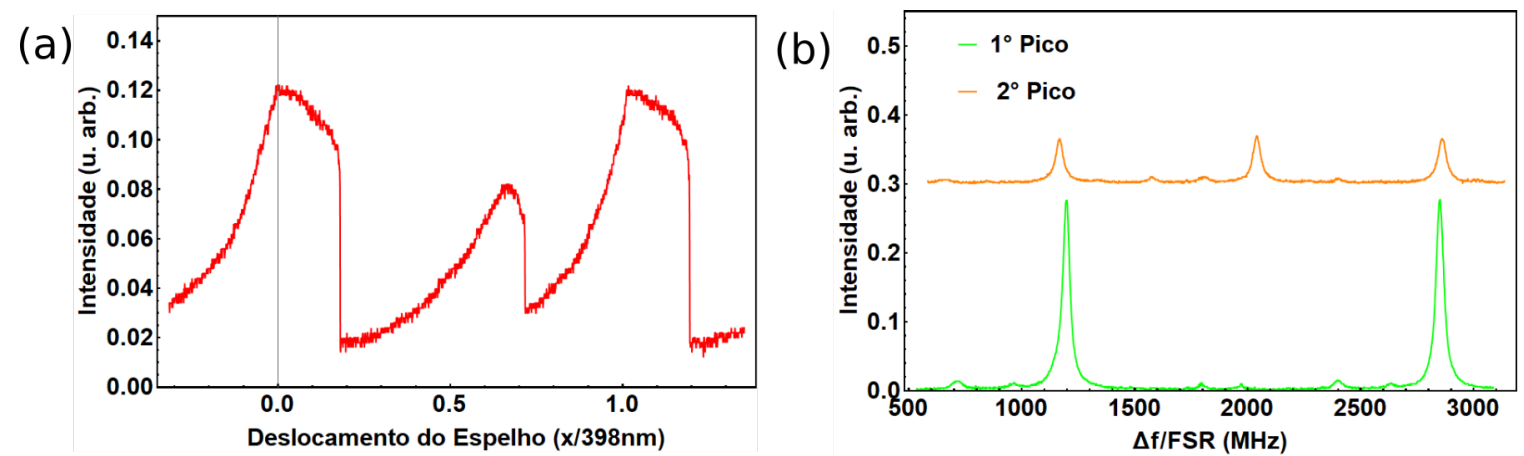

Figura 4.5: (a) Intensidade dos campos gerados pelo OPO quando o comprimento $\mathrm{x}$ da cavidade do OPO é varrido. Comprimento dado unidades de meio comprimento de onda (x/398 nm). (b) Situação em que o OPO é travado em um de seus picos característicos, e o campo gerado pelo OPO passa por uma cavidade em anel juntamente com o campo de bombeio. O sinal verde se refere a intensidade do campo quando travamos o OPO no primeiro pico, o sinal laranja se refere intensidade do campo ao travar o OPO no segundo pico. Essas intensidade são dadas em função da varredura da frequência de ressonância $\Delta \mathrm{f}$ da cavidade em anel normalizada pelo FSR.

(a)

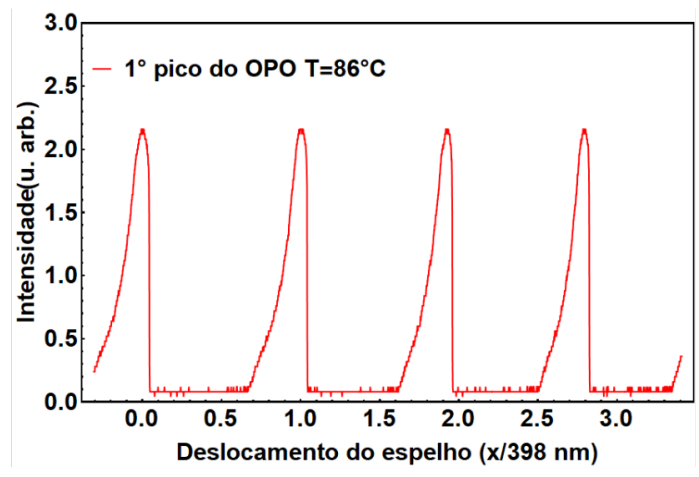

$260 \mathrm{MHz}$

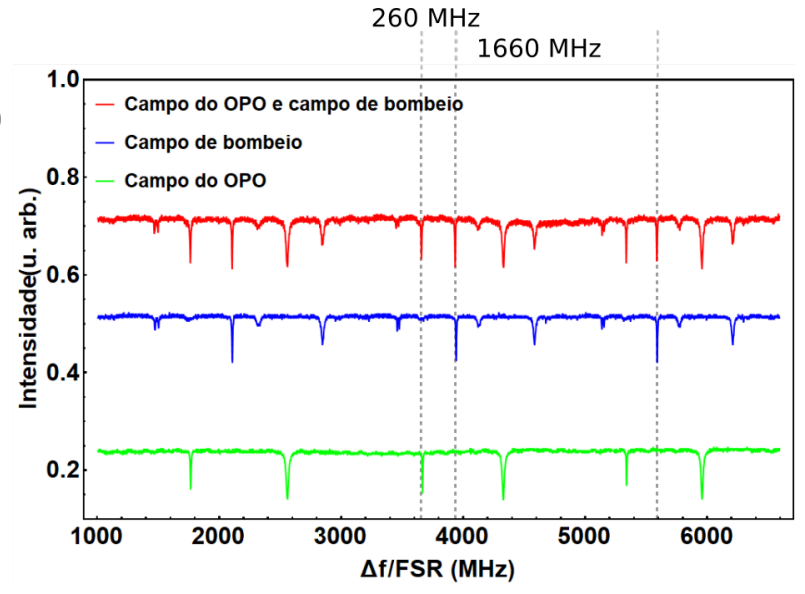

Figura 4.6: (a)Intensidade do campo referente ao primeiro pico gerado pelo OPO quando sua cavidade é varrida e a célula de rubídio mantida a $86^{\circ}$ e $\mathrm{F}=37,8$ em função da varredura do comprimento $\mathrm{x}$ da cavidade do OPO. Comprimento dado unidades de meio comprimento de onda $(\mathrm{x} / 398 \mathrm{~nm})$. (b) Situação em que o OPO é travado no $1^{\circ}$ pico, e o campo referente a esse pico passa por uma cavidade em anel juntamente com o campo de bombeio. O gráfico mostra as intensidades da sobreposição entre campo do OPO e bombeio (vermelho), apenas campo de bombeio (azul) e apenas campo do OPO (verde). Essas intensidade são dadas em função da varredura da frequência de ressonância $\Delta \mathrm{f}$ da cavidade em anel normalizada pelo FSR.

A ausência de interferência nos leva a testar a hipótese de que o campo referente ao $1^{\circ}$ pico e o campo do bombeio encontram-se em frequências diferentes. Assim, fazemos uma parcela 
do campo do bombeio (ou campo do Ti:safira) e o campo de prova gerado pelo OPO passarem pela mesma cavidade em anel. O resultado da superposição dos campos do OPO e do bombeio é apresentado na figura 4.6. Nessa figura são apresentadas três medidas, em vermelho temos os picos gerados pela sobreposição do campo do OPO e o campo do bombeio, em azul, picos gerados pelo campo do bombeio, em vermelho, picos gerados campo do OPO. A sobreposição entre as três medidas não é perfeita pois os dados do osciloscópio sofrem uma pequena deriva entre uma medida e outra.

Para fazer essa medida da sobreposição entre os campos do OPO e campo de bombeio precisamos contornar um pequeno problema, a parcela do campo de bombeio que estamos enviando a cavidade em anel é horizontalmente polarizada e o campo de saída do OPO é verticalmente polarizado. Dessa forma, utilizamos uma placa de $\lambda / 2$ e uma placa de $\lambda / 4$ colocada na entrada da cavidade em anel para fazer com que as polarizações do campo do OPO e do campo de bombeio se tornem mistas.

A cavidade em anel possui finesses diferentes para campos com polarização horizontal e vertical. Campos que geram picos mais estreitos possuem polarização vertical enquanto campos mais largos possuem polarização horizontal. O intervalo espectral livre da cavidade em anel utilizada nesse momento é de $\Delta \omega=1660 \mathrm{MHz}$, e medimos a distância entre dois picos consecutivos do bombeio no osciloscópio, $D$, e a distância entre um pico do campo de bombeio e o campo dessintonizado, $d$. Sabendo que $D$ é proporcional ao intervalo espectral livre, podemos estimar a dessintonia mínima entre bombeio e campo gerado por $x=\Delta \omega \cdot d / D$. Dessa forma, obtivemos que os dois campos encontram-se dessintonizados por:

$$
\Delta=(1660 n \pm 260) \mathrm{MHz}
$$

onde $n=0,1,2, \ldots$ Assim, é possível concluir que o fenômeno em questão não é o $4 \mathrm{WM}$ degenerado.

Uma hipótese plausível para explicar essa dessintonia $\Delta$ é que ela seja fruto de uma transição Raman. Essa transição se caracteriza por um espalhamento inelástico dos fótons absorvidos pelo material, ou seja, o fóton emitido pelo material possui frequência menor que a frequência do fóton incidente. Se na equação 4.2 temos $n=2$ e o sinal \pm é negativo, concluímos que $\Delta=3052 \mathrm{MHz}$. Uma transição Raman de geometria $\lambda$ entre os níveis hiperfinos $5 S_{1 / 2} \mathrm{~F}=2$ e $5 S_{1 / 2} \mathrm{~F}=3$ e o nível excitado $5 P_{1 / 2}$ da linha $\mathrm{D} 1$ do rubídio deve espalhar um fóton de frequência $3052 \mathrm{MHz}$. Considerando que as medidas possuem um erro de $\pm 16,6 \mathrm{MHz},(1 \%$ do FSR da cavidade triangular) podemos concluir que a dessintonia entre o campo ejetado pelo OPO e o campo de bombeio é o que se espera se o campo ejetado é proveniênte de uma transição Raman.

Contudo, ainda não temos certeza de que o fenômeno que gera o $1^{\circ}$ pico é o efeito Raman. 


\subsubsection{Campos gerados para célula de rubídio à $60^{\circ} \mathrm{C}$}

Devido à descoberta desse campo dessintonizado do bombeio ( $1^{\circ}$ pico), decidimos investigar a dependência desse fenômeno com a temperatura e descobrimos que a frequência dos campos gerados é sensível a mesma. Para temperaturas menos elevadas, na faixa de $60^{\circ} \mathrm{C}$, o $\mathrm{OPO}$ apresenta dois novos modos de oscilação, os quais apresentamos na figura 4.7. Nessa figura vemos os picos $1^{\circ}$ e $2^{\circ}$ gerados pelo OPO a $60^{\circ}$ na parte (a), na parte (b) mostramos a sobreposição do campo referente ao primeiro modo que oscila no OPO (pico $1^{\circ}$ ) e o bombeio enquanto a parte (c) mostramos sobreposição entre o campo do segundo pico a oscilar (pico $2^{\circ}$ ) e o bombeio. Lembramos que no intervalo entre as medidas os dados do osciloscópio sofrem uma pequena deriva, de modo que a sobreposição entre as três diferentes medidas não é perfeita.

$\mathrm{O} 1^{\circ}$ pico a oscilar possui a mesma frequência que o bombeio, como podemos deduzir da figura 4.7 (b) onde vemos a sobreposição entre o campo de bombeio e o campo do OPO. Uma hipótese que explica esses campos possuírem a mesma frequência é de que o campo do OPO é produzido por 4WM degenerado.

Observando a figura 4.7 (b) concluímos que o $2^{\circ}$ pico a oscilar possui uma dessintonia de $( \pm 621+1660 n) \mathrm{MHz}$ em relação ao campo de bombeio, onde $n=0,1,2, \ldots$. Não encontramos nenhum fenômeno que possa explicar a essa dessintonia.

Além de variar a temperatura, experimentamos variar a finesse e mexer no alinhamento da célula de rubídio. Constatamos que não há mudança significativa dos picos quando mudamos a finesse, mas podemos fazer com que algum dos modos do OPO deixem de oscilar mexendo no alinhamento. 
(b)

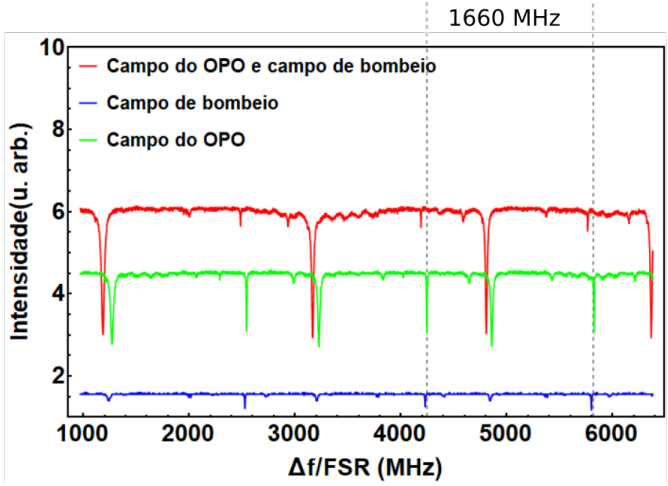

(a)

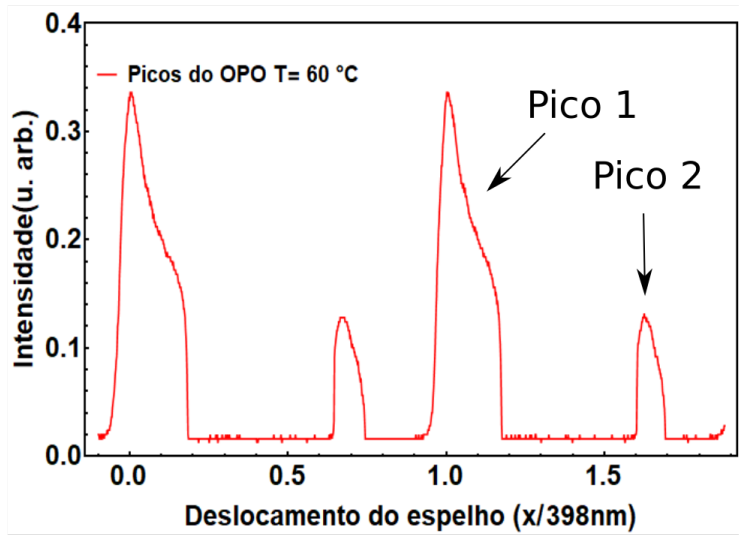

(c) Sobreposição do Pico 2 e bombeio $1660 \mathrm{MHz}$ $621 \mathrm{MHz}$

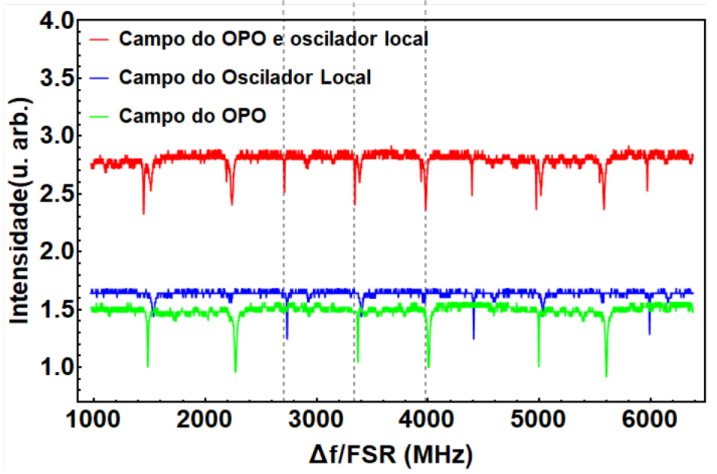

Figura 4.7: (a)Intensidade do campo referente ao primeiro pico gerado pelo OPO quando sua cavidade é varrida e a célula de rubídio mantida a $60^{\circ} \mathrm{F}=37,8$ em função da varredura do comprimento $\mathrm{x}$ da cavidade do OPO. Comprimento dado unidades de meio comprimento de onda (x/398 nm). (b) Situação em que o OPO é travado no $1^{\circ}$ pico, e o campo referente a esse pico passa por uma cavidade em anel juntamente com o campo de bombeio. (c) Situação em que o OPO é travado no $1^{\circ}$ pico, e o campo referente a esse pico passa por uma cavidade em anel juntamente com o campo de bombeio. Os gráficos (b) e (c) mostram as intensidades da sobreposição entre campo do OPO e bombeio (vermelho), apenas campo de bombeio (azul) e apenas campo do OPO (verde). Essas intensidade são dadas em função da varredura da frequência de ressonância $\Delta \mathrm{f}$ da cavidade em anel normalizada pelo FSR.

\subsection{Caracterização da cavidade de separação}

Quando estamos na situação em que os campos gerados pelo OPO se devem a 4WM não degenerado precisamos separar os campos de prova e conjugado para realizar as medidas de detecção homodina ou deteçã̃o balanceada de forma separada. Para essa tarefa utilizamos uma cavidade de separação.

A cavidade de separação é uma cavidade triangular que possui a função de separar campos de frequências distintas. De tal forma que a cavidade reflete um dos campos e transmite o outro. Para cumprir essa função, a largura de banda da cavidade deve possuir duas características 
especiais:

- A largura de banda da cavidade de filtro deve ser menor que a metade da diferença entre as frequências dos campos de prova e conjugado que desejamos separar. Dessa forma, garantimos que a informação contida nas bandas laterais desses dois campos não se misture. Sabemos que a separação entre os dois campos é de $6 \mathrm{GHz}$, portanto, $\Delta \omega_{\text {filtro }} \leq 3$ GHz.

- A largura de banda da cavidade de filtro deve ser maior que a frequência de análise que utilizamos na detecção homodina (ou na detecção balanceada), visto que de outra forma estaríamos descartando informação proveniente do OPO. No caso do nosso sistema, medimos entre $500 \mathrm{KHz}$ e $20 \mathrm{MHz}$.

A cavidade de filtro utilizada no experimento real possui largura de banda de $\Delta \omega_{\text {filtro }}=$ $46 \mathrm{MHz}$ para polarização vertical e $\Delta \omega_{\text {filtro }}=71,68 \mathrm{MHz}$ para campos que incidem com polarização horizontal. No experimento optamos por utilizar campos com polarização vertical e para garantir que todo o campo na entrada da cavidade possua polarização vertical, utilizamos uma placa de $\lambda / 2$ em conjunto com uma placa de $\lambda / 4$ para ajustar essa condição.

A cavidade foi construída com um espelho HR de raio de curvatura $R=500 \mathrm{~mm}$ e dois espelhos planos de $1 \%$ de transmissão. O espelho HR está preso a um PZT que vibra e varia o comprimento da cavidade. Quando a cavidade está ressonante com o campo conjugado, esse campo é transmitido e o campo de prova é refletido. Travamos a cavidade nessa condição, e dessa forma, a cavidade age separando os dois campos.

Na tabela 4.5 destacamos as principais características da cavidade de filtro:

\begin{tabular}{|l|l|}
\hline Características da cavidade de separação & \\
\hline FSR & $1,66 \mathrm{GHz}$ \\
\hline Finesse para polarização horizontal & 36 \\
\hline Finesse para polarização vertical & 199 \\
\hline Largura de banda para ploarização horizontal & $46 \mathrm{MHz}$ \\
\hline Largura de banda para ploarização vertical & $71,68 \mathrm{MHz}$ \\
\hline
\end{tabular}

\subsection{Caracterização do $1^{\circ}$ pico à célula de rubídio à $\mathrm{T}=86^{\circ} \mathrm{C}$}

Iniciamos o a caracterização dos campos produzidos com o estudo das flutuações do campo referente ao $1^{\circ}$ pico do OPO. Devido à dessintonia de $(1660 n \pm 260) \mathrm{MHz}$ em relação ao campo do bombeio não podemos utilizar uma fração do campo de bombeio como oscilador local. Sendo assim, optamos por utilizar a cavidade triangular do experimento como cavidade de análise e aplicar a técnica da rotação da elipse de ruído para caraterizar esse campo.

Fizemos um amplo estudo das flutuações do campo do $1^{\circ}$ pico utilizando a técnica da rotação de elipse. Investigamos o ruído em frequências de análise de 9MHz, $10 \mathrm{MHz}$ e $11 \mathrm{MHz}$. Para 
cada uma dessas frequências de análise variamos a potência de bombeio entre $18 \mathrm{~mW}$ e $47 \mathrm{~mW}$. Em todos os casos encontramos um campo aproximadamente coerente ou com um pequeno excesso de ruído. Na figura 4.8 apresentamos o resultado desses testes para uma potência do bombeio de $18 \mathrm{~mW}$ e frequências de análise de $9 \mathrm{MHz}, 10 \mathrm{MHz}$ e $11 \mathrm{MHz}$.

Quando trabalhamos com a técnica da rotação da elipse de ruído precisamos garantir que a frequência de análise normalizada pela largura de banda, $\Omega^{\prime}=\Omega /\left(\Delta \Omega_{\text {CavAn }}\right)$, seja menor que $\sqrt{2}$. Caso contrário, não haverá o giro completo da elipse de ruído, o que gera valores subestimados para a potência do ruído de fase [17]. Como as frequências de análise utilizadas no trabalho são pequenas, necessitamos modificar a cavidade de tal forma que sua largura de banda diminua. Assim, trocamos um dos espelhos de transmissão de 1\% por um espelho plano com coating para $780 \mathrm{~nm}$ da empresa Thorlabs. O comportamento da cavidade modificada é tal que a largura de banda da cavidade é de 7,1 MHz para polarização horizontal e de 27,71 $\mathrm{MHz}$ para polarização horizontal. Para garantir essa condição, utilizamos uma placa de $\lambda / 2$ e $\lambda / 4$ para ajustar a polarização do campo na entrada da cavidade.

As principais características da cavidade modificada são listadas na tabela 4.6:

\begin{tabular}{|l|l|}
\hline Características da cavidade de análise & \\
\hline FSR & $1,66 \mathrm{GHz}$ \\
\hline Finesse para polarização horizontal & 46 \\
\hline Finesse para polarização vertical & 232 \\
\hline Largura de banda para polarização horizontal & $27,71 \mathrm{MHz}$ \\
\hline Largura de banda para polarização vertical & $7,1 \mathrm{MHz}$ \\
\hline
\end{tabular}

As frequências de análise normalizadas pela largura de banda da cavidade modificada são: $\Omega^{\prime}=1,26,1,40$ e 1,54 . Para a frequência de $\Omega^{\prime}=1,26$ as medidas da potência de ruído da fase estão subestimadas.

Como dito anteriormente, uma hipótese para explicar a dessintonia entre bombeio e campo do OPO é de que esteja ocorrendo espalhamento Raman. Apenas com o estudo das flutuações desse campo não foi possível chegar a uma conclusão sobre o fenômeno que está gerando esse campo. No entanto, sabemos que esse campo é um estado coerente e a intenção desse trabalho é estudar sistemas que apresentem características quânticas tais como compressão de ruído e emaranhamento. Lembrando que o OPO gera dois outros campos diferentes, decidimos mudar o foco do nosso estudo e investigar os demais campos. 


\section{Rotação da Elipse de Ruído}
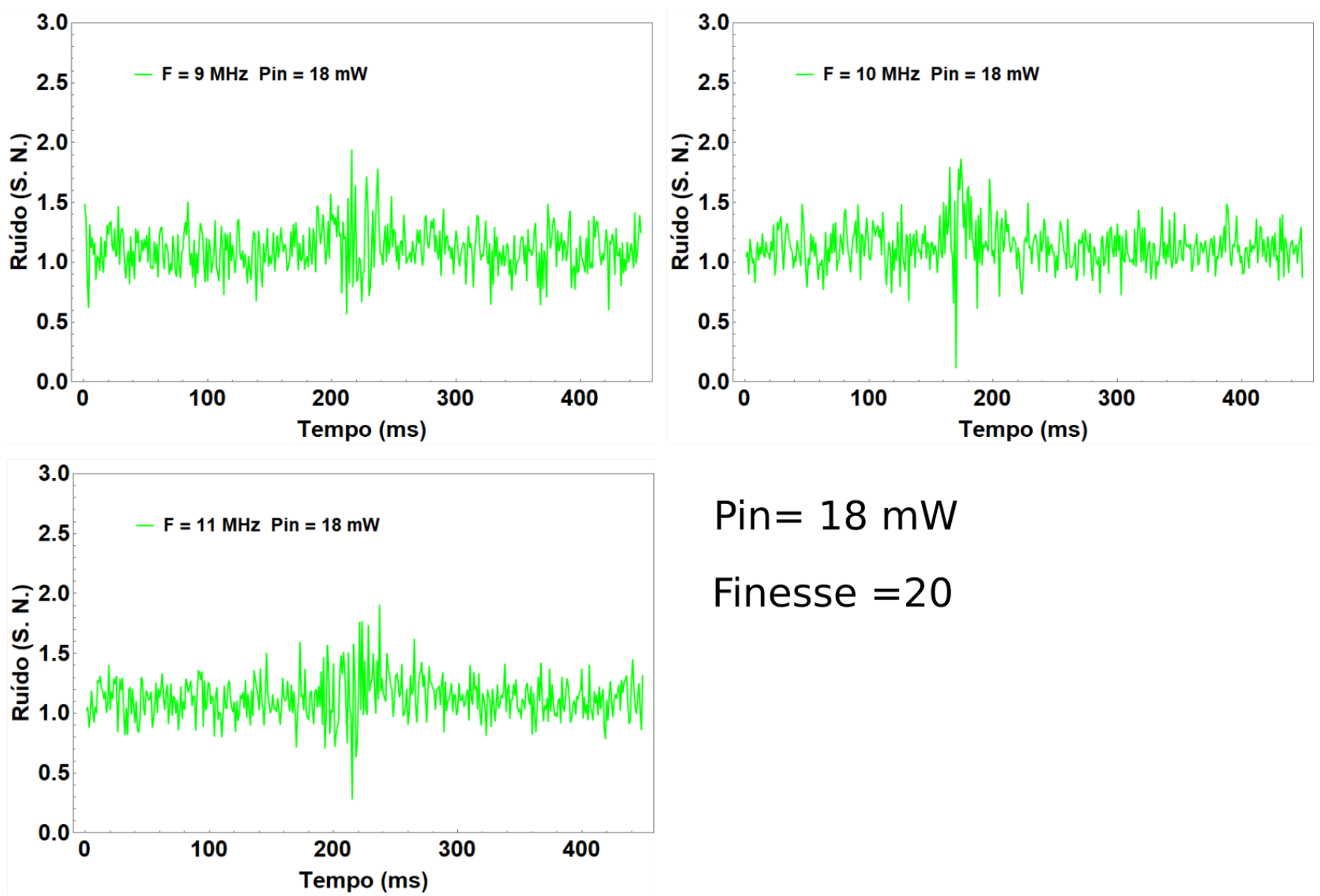

$\operatorname{Pin}=18 \mathrm{~mW}$

Finesse $=20$

Figura 4.8: Resultado da rotação da elipse de ruído para o campo do $3^{\circ}$ do OPO com finesse de 20 e potências de entrada do bombeio de $18 \mathrm{~mW}$ nas frequencias de análise de $9 \mathrm{MHz}, 10 \mathrm{~Hz}$ e $11 \mathrm{MHz}$.

\subsection{Caracterização do $2^{\circ}$ pico $\mathrm{T}=60^{\circ} \mathrm{C}$}

Utilizamos a detecção homodina para caracterizar o campo referente ao $2^{\circ}$ pico produzido pelo OPO à $60^{\circ}$. Como foi visto anteriormente, esse campo possui a mesma frequência que o campo de bombeio, o que nos leva a acreditar que está sendo produzido por meio do processo de 4WM degenerado. Nesse caso, os campos de prova e conjugado são produzidos com frequências iguais e não temos como separa-los com a cavidade de separação. O que fazemos é bloquear o espelho com PZT da cavidade de separação, dessa forma apenas $1 \%$ da potência do nosso campo é perdida no espelho de entrada dessa cavidade mas o restante da potência é refletido para o sistema de detecção homodina. Como não podemos separar os campos de prova e conjugado, qualquer técnica de interferometria utilizada só poderá medir as quadraturas do espaço da soma dos campos de prova e conjugado.

Visto que só podemos medir o espaço da soma, devemos nos perguntar que informação 
podemos obter com essa medida. Sabemos que no processo de 4WM aniquilamos dois fótons do bombeio e produzimos outros dois fótons nos modos de prova e conjugado. Quando consideramos os campos emitidos pelo OPO em uma situação ideal, sem perdas, esperamos que o número de fótons do campo de prova seja igual ao número de fótons do modo conjugado levando a uma compressão de ruído perfeita na quadratura de diferença ente as amplitudes dos modos de prova e conjugado e na quadratura da soma das fases desses mesmos campos. Assim, esperávamos que o campo produzido pelo $2^{\circ}$ pico do OPOà $60^{\circ}$ apresentasse compressão de ruído na quadratura da soma das fases.

$\mathrm{Na}$ figura 4.9 apresentamos as medidas de detecção homodidina para o campo de prova gerado pelo OPO oscilando no modo do 4WM degenerado com potência de bombeio de 3,8 $\mathrm{mW}$ e finesse de 37,8 para três fases relativas diferentes entre o oscilador local e o campo de prova. Podemos ver que independente da fase relativa entre os campos o ruído do campo de prova se encontra próximo ao ruído quântico padrão. Também foram feitas outras medidas para diferentes potências de bombeio e duas finesses distintas, 37,8 e 8. Em todos os casos, observamos o ruído do campo de prova próximo ao ruído quântico padrão. Isso significa que o campo em questão está em um estado coerente assim como o campo de bombeio.

Algumas hipóteses que podem justificar esse resultado são:

- Efeitos produzidos pela interação de campos contrapropagantes na célula de rubídio. Em nossa cavidade linear o campo de bombeio passa duas vezes pela célula de rubídio. Quando o 4WM é produzido pela aniquilação de um fóton propagante e um fóton contra propagante, os campos produzidos são resultado de termos que não foram contados no Hamiltoniano 3.1. Para obtermos o melhor ganho possível do 4WM precisamos realizar o experimento a uma certa dessintonia em relação ao bombeio.

- Outra possibilidade é que ao dessintonizar o bombeio a linha hiperfina do rubídio que estávamos utilizando, tenhamos travado o OPO muito próximo a ressonância com a próxima linha hiperfina gerando efeitos de absorção. 


\section{Detecção homodina para o caso degenerado}
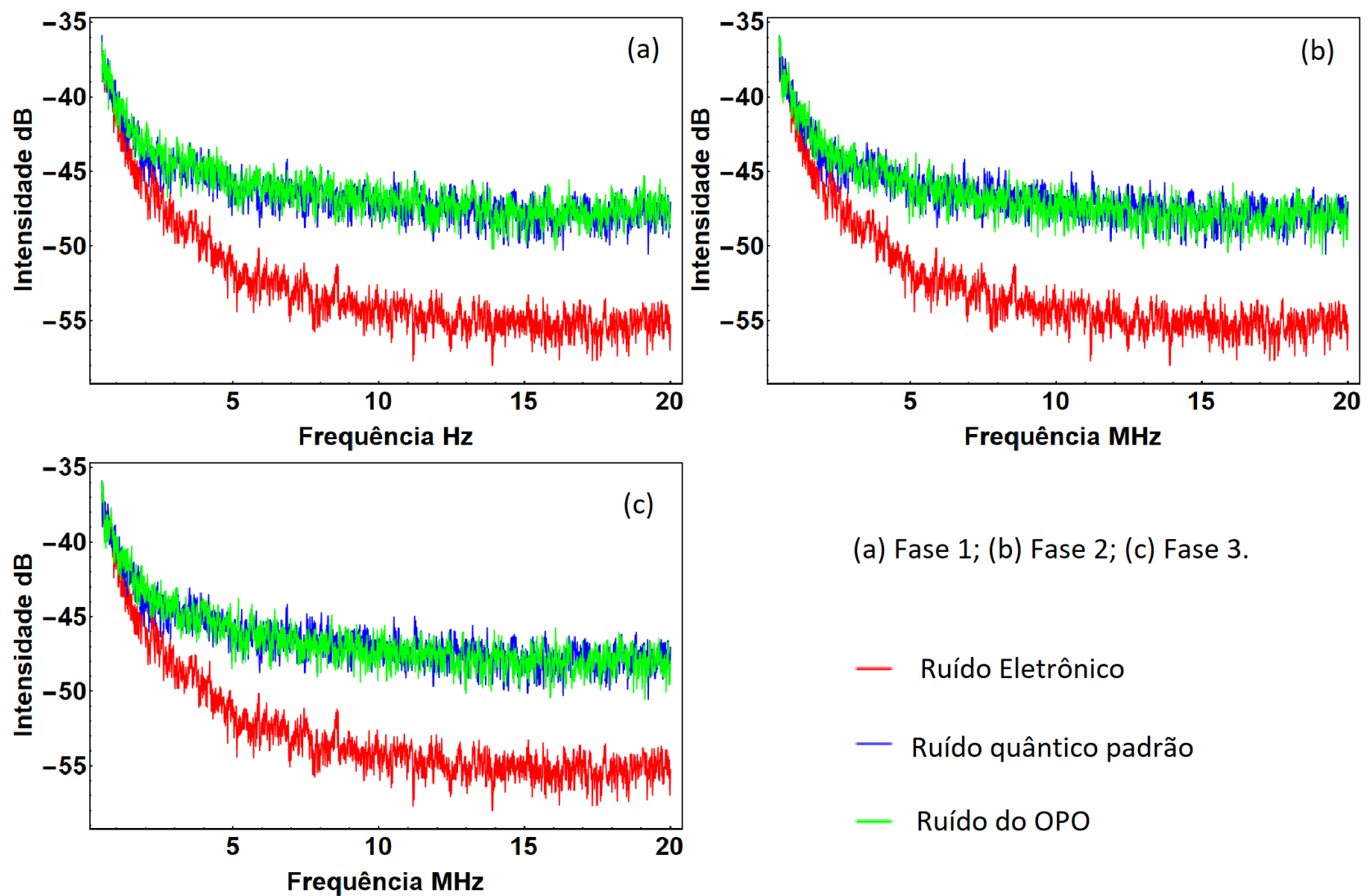

(a) Fase 1; (b) Fase 2; (c) Fase 3.

- Ruído Eletrônico

— Ruído quântico padrão

- Ruído do OPO

Figura 4.9: Ruído do OPO utilizando a técnica da detecção homodina em função da frequência de análise. O experimento foi realizado com a cavidade do OPO com a uma finesse de 37,8 oscilando no modo do 4WM degenerado com potência do bombeio de $38 \mathrm{~mW}$ e para três fases relativas diferentes entre o oscilador local e o campo de prova.

\subsection{Caracterização dos detectores disponíveis para as me- didas do campo do $2^{\circ}$ pico $\mathrm{T}=86^{\circ} \mathrm{C}$}

Como descrito anteriormente, o $2^{\circ}$ pico do OPO é resultado de 4WM não degenerado. Para caracterizar seu ruído necessitamos de quatro detectores iguais para poder realizar medidas de detecção balanceada nos campos de prova e conjugado separadamente. Cada par de detectores deve ter ganho suficiente para que possamos diferenciar facilmente a soma e diferença dos ruídos HF medidos por cada par de detectores do ruído eletrônico dos mesmos.

O máximo de potência que conseguimos fornecer ao OPO são $52 \mathrm{~mW}$, e com essa potência os campos de prova e conjugado criados quando travamos o OPO no segundo pico possuem uma potência muito baixa de no máximo $490 \mu \mathrm{W}$ cada um. Infelizmente, a caracterização dos detectores disponíveis no laboratório revelou que os mesmos não possuem ganho suficiente para 
realizar as medidas de campos de tão baixa intensidade. Sendo assim, será necessário fazer modificações nos circuitos eletrônicos dos mesmos e uma nova caracterização.

Devido a proximidade do encerramento do prazo de depósito dessa tese, necessitei encerrar aqui o estudo do experimento para poder me dedicar a redação da tese. Sendo assim, encerro o capítulo experimental com perspectivas para o estudo do 4WM não degenerado:

- Quando o problema dos ganhos dos detectores for resolvido, já será possível fazer medidas de detecção balanceada. Esperamos que essas medidas revelem compressão de ruído da diferença entre as quadraturas de amplitude dos campos de prova e conjugado.

- Existem planos para a compra de um novo modulador acusto-ótico (acusto-optic aodulator $\mathrm{AOM}$ ) que deve ser capaz de deslocar a frequência do bombeio de $3 \mathrm{GHz}$ e produzir um campo na mesma frequência que o campo conjugado. Assim, será possível realizar medidas de deteção homodina e ter acesso tanto a quadratura amplitude quanto a quadratura fase dos campos de prova e conjugado. Esperamos que essas medidas confirmem as previsões teóricas de compressão de ruído e emaranhamento do capítulo 3.

- Também existem planos de deslocar o laser Ti:safira para a mesa do experimento. Dessa forma, será possível explorar o comportamento dos campos gerados quando a potência de entrada do OPO é mais alta. 


\section{Capítulo 5}

\section{Conclusões e perspectivas}

Nessa tese, resolvemos o modelo teórico do OPO $\chi^{(3)}$ duplamente ressonante (prova e conjugado) e triplamente ressonante (bombeio, prova e conjugado). Nesse modelo apresentamos a solução dos campos médios e estudamos as flutuações quânticas dos campos na operação acima do limiar de oscilação para os campos de ressonância dupla e tripla. Sendo que a resolução dos campos médios é um resultado inédito e apesar do método utilizado para caracterizar as flutuações do sistema já ser muito bem estabelecido [53], a aplicação desse método ao nosso sistema produz a caracterização das flutuações dos sistemas DROPO e TROPO $\chi^{3}$, o que também é inédito. O estudo das flutuações é feito considerando a descrição gaussiana e, por meio da matriz de covariância, fizemos as previsões teóricas de compressão de ruído e emaranhamento do sistema.

Como principais resultados podemos destacar a previsão de compressão de ruído da fase do bombeio do sistema DROPO, compressão de ruído de amplitude dos campos de prova e conjugado que aparece tanto no DROPO quanto no TROPO e a rica estrutura de emaranhamento vista nos dois sistemas.

Vale à pena destacar alguns resultados da caracterização do emaranhamento dos sistemas DROPO e TROPO. Vimos que no caso do DROPO temos violação do critério de Duan para prova e conjugado em uma região que se estende de $1 \leq \sigma \leq 2,22$ enquanto que no caso do TROPO essa região de emaranhamento se estende de $1 \leq \sigma \leq 6$, a maior região de violação do critério de Duam do modelo do TROPO possibilita a implementação de protocolos de teletransporte em uma faixa de potência maior que o DROPO.

Por meio do teste PPT fizemos uma caracterização de emaranhamento em um sistema tripartite formado pela parte simétrica dos campos de bombeio, prova e conjugado. No caso do DROPO temos emaranhamento tripartite na região entre $1 \leq \sigma \leq 2,8$, enquanto no caso do TROPO temos emaranhamento tripartite na região $1,53 \leq \sigma \leq 4$. Também caracterizamos o sistema hexapartite que considera a matriz de covariância completa do sistema. Vale a pena destacar que as partes simétrica e antissimétrica do sistema encontram-se emaranhadas em toda a região analisada mas com exceção de um ponto, $\sigma=2$ para o DROPO e $\sigma=4$ para o TROPO, estão emaranhadas. Além disso, as bipartições $2 \times 4$ e 2x2 entre as partes simétrica e 
antissimétrica do sistema estão emaranhadas em toda a região analisada.

Futuramente, o modelo teórico será estendido para a análise de momentos de ordens superiores, ou seja, considerando o estado não gaussiano.

Apesar do experimento implementado nesse projeto não ter apresentado os resultados esperados, ele foi uma etapa importante para detectarmos problemas que necessitam ser resolvidos. O experimento consistiu de um OPO $\chi^{3}$ cuja cavidade é linear e o meio de ganho é uma célula de vapor de rubídio. Esse sistema produziu não produziu apenas os campos de 4WM esperados. Conforme variamos temperatura e alinhamento da célula o OPO produz quatro picos diferentes.

O primeiro pico produzido pelo $\mathrm{OPO}$ à $86^{\circ}$ corresponde a um campo que está dessintonizado de pelo menos $1660 n \pm 260 \mathrm{MHz}$ em relação ao campo de bombeio produzido pelo Ti:safira. Provavelmente, o fenômeno que gera esse campo é espalhamento Raman. Estudamos o ruído produzido por ele. Por meio da técnica da rotação da elipse de ruído determinamos que o ruído desse campo é compatível com um estado coerente.

$\mathrm{O}$ pico produzido pelo $\mathrm{OPO}$ com a cúlula de rubídio à $60^{\circ} \mathrm{C}$ corresponde ao campo do $4 \mathrm{WM}$ degenerado e seu ruído foi estudado por meio da técnica da detecção homodina e as medidas de ruído desse sistema mostraram compatíveis com um estado coerente.

O segundo pico produzido pelo $\mathrm{OPO}$ com a cúlula de rubídio à $86^{\circ} \mathrm{C}$ corresponde a $4 \mathrm{WM}$ não degenerado. Devido a dificuldades técnicas com os detectores, não houve tempo para a caracterização desse sistema.

As conclusões mais relevantes sobre formas de melhorar o experimento provém do estudo do 4WM degenerado. Caso esteja havendo efeitos provocados por campos contra-propagantes podemos evitar esses efeitos substituindo a cavidade linear por uma cavidade triangular. Também existe a possibilidade de que ao dessintonizar o bombeio da linha hiperfina que estamos usando tenhamos feito o bombeio entrar em ressonância com outra linha hiperfina próxima, assim, outra ideia é medir com o bombeio dessintonizado de outra linha hiperfina.

O estudo do OPO $\chi^{(3)}$ é novo no grupo mas possui grandes perspectivas, por meio do estudo do limiar do OPO espera-se fazer a caracterização teórica e experimental dos estados não gaussianos gerados. Além disso, existe a possibilidade de se criar uma rede de informação quântica entre esse experimento e outros experimentos do laboratório que possam interagir com as linhas D1 (794.98 nm) do rubídio. 


\section{Apêndice A}

\section{Dados ATFilms}

Espelhos da cavidade do OPO

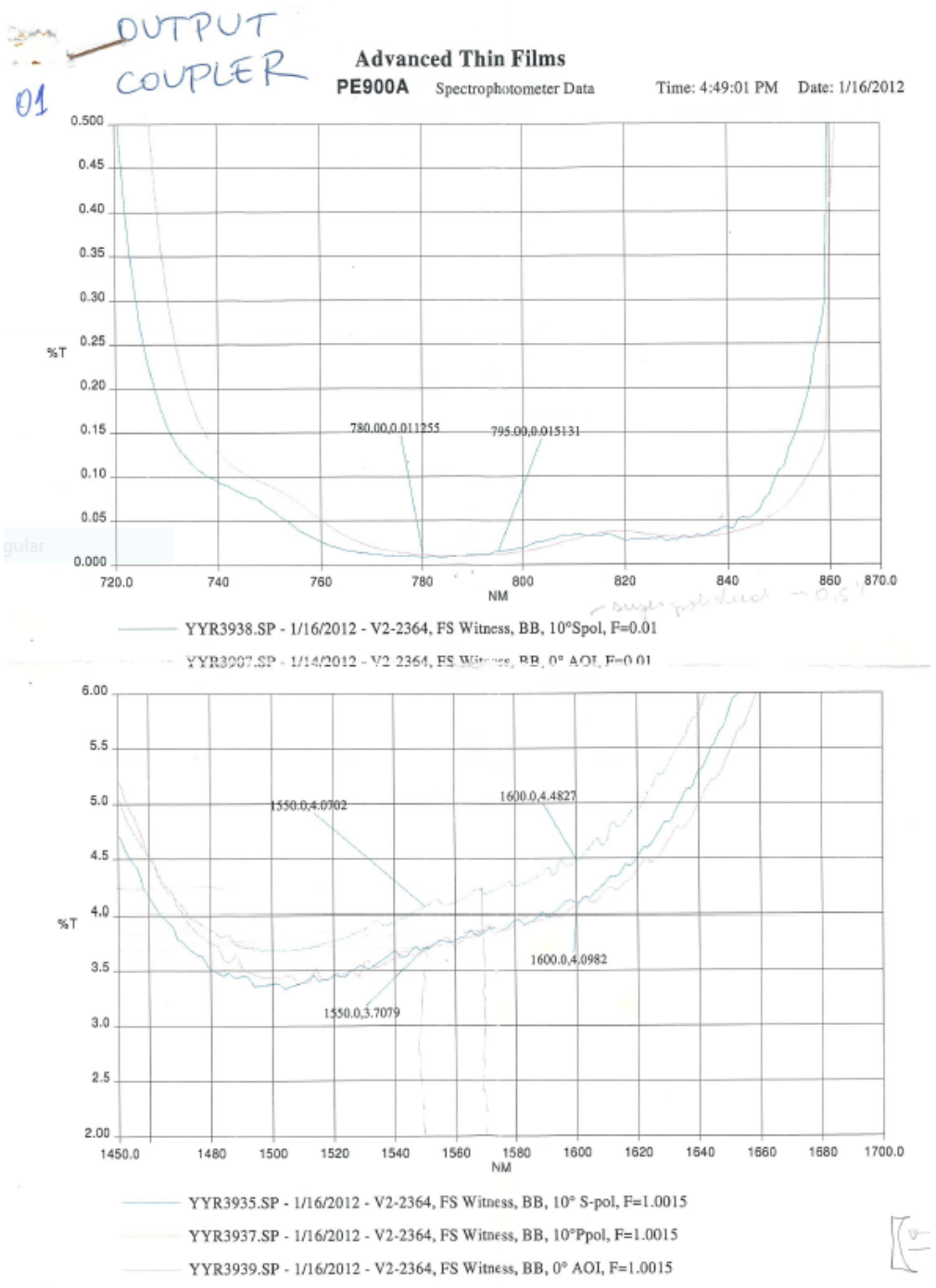

Figura A.1: ATFilms (v2-2364 \& v2-2367) 


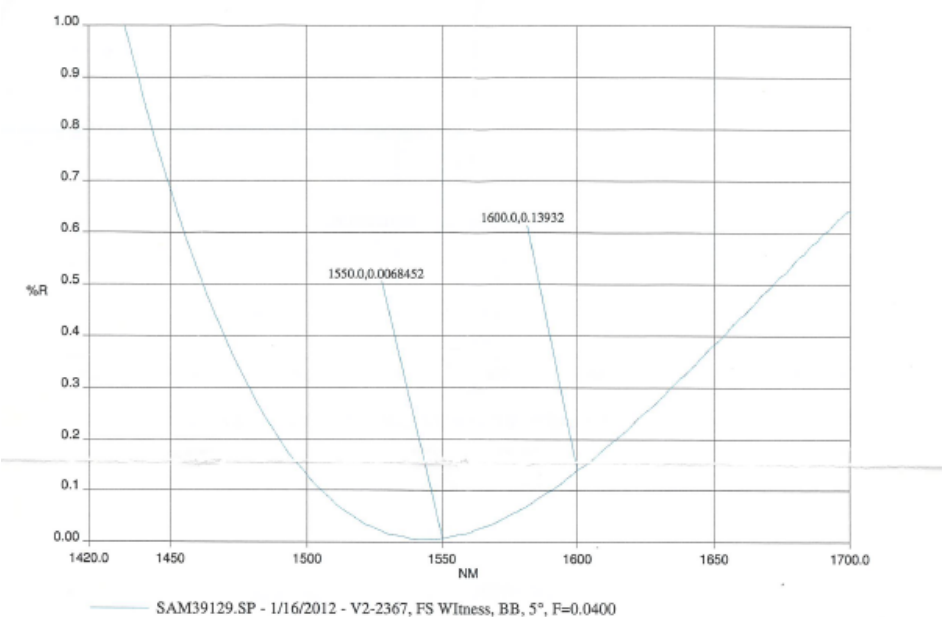

Figura A.2: ATFilms (v2-2364 \& v2-2367) 


\section{Apêndice B}

\section{Geração da dessintonia}

Para atingir grandes dessintonias fazendo uso de AOMs (acousto optic modulator) de pequena frequência é necessário fazer com que o feixe de luz passe por ele diversas vezes. A cada passagem da luz pelo AOM o feixe se abre em ordens de difração. Essas ordens de difração alteram a frequência do feixe de forma que um feixe que entre no AOM com uma frequência $f$ sairá com frequência $f+\alpha n$ onde $\mathrm{n}$ é a ordem de difração e $\alpha$ é a dessintonia que o AOM causa a cada passagem, no nosso caso 200MHz. A Figura B.1 mostra o circuito montado para fazer com que o feixe perca $800 \mathrm{MHz}$ ao final do processo.

Na Figura B.1 ilustramos cada etapa do processo, porém omitimos uma lente que forma um telescópio para simplificar o desenho. Na primeira passagem o laser passa pelo isolador com polarização horizontal e frequência $f$, atravessa o PBS e faz a primeira passagem pelo AOM, selecionamos a ordem de difração -1 e bloqueamos as demais ordens. Agora o feixe possui frequência $f-200 M H z$, passa pela placa de $\lambda / 4$ e chega ao espelho.

A luz que bate no espelho e volta passa novamente pela placa de $\lambda / 4$. Ele faz sua segunda passagem pelo AOM e selecionamos a ordem -1 novamente. O feixe, agora, possui frequência $f-400 M H z$ e polarização vertical por ter passado duas vezes pela placa de $\lambda / 4$. Sendo assim o feixe é refletido pelo PBS e atinge o espelho.

Após atingir o espelho, o feixe retorna pelo mesmo caminho e passa novamente pelo A.O.M., selecionamos então a ordem de difração -1. O feixe agora possui frequência $f-600 \mathrm{MHz}$. Ele passa pela placa de $\lambda / 4$ e é refletido novamente pelo espelho, voltando pelo mesmo caminho.

Após passar pela placa de $\lambda / 4$ o feixe adquire polarização horizontal, passa novamente pelo A.O.M., selecionamos a ordem de difração -1 e agora, o feixe possui frequência $f-800 \mathrm{MHz}$, que é a frequência desejada. Ele atravessa o P.B.S. e em seguida o isolador ótico sendo expulso pela porta lateral indicada na Figura B.1. 

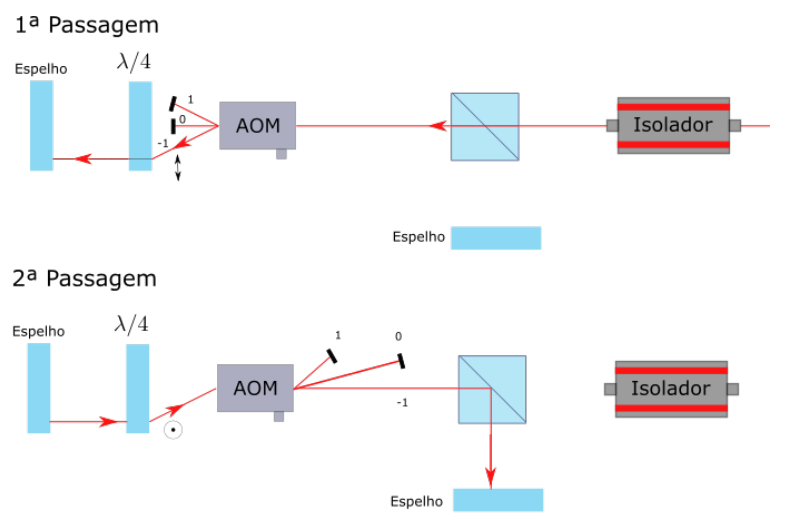

$3^{a}$ Passagem

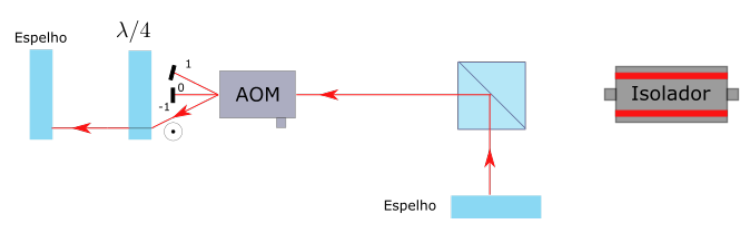

$4^{a}$ Passagem

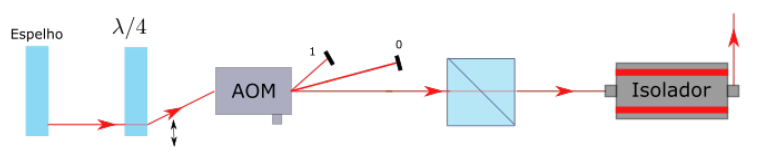

Espelho

Figura B.1: A figura mostra como fazer com que um feixe passe quatro vezes por um AOM. Tal processo é utilizado para atingir grandes dessintonias mesmo quando se dispõe apenas de um AOM de frequência baixa. Trata-se de um jogo de mudança de polarização e reflexões que será explicado no texto. 


\section{Apêndice C}

\section{Absorção saturada}

Essa seção é uma descrição sucinta do experimento de absorção saturada que foi usado nesse experimento para sintonizar nossos lasers nas transições desejadas. O texto [60] foi usado como referencia para essa seção.

A Figura C.1 mostra o circuito de absorção saturada. Nessa figura, podemos ver um feixe de luz proveniente de um laser sendo dividido pelo PBS em duas partes, uma mais intensa e de polarização vertical (feixe em vermelho) e um feixe de luz de menor intensidade e polarização horizontal (feixe preto). Esses feixes são sobrepostos e atravessam a célula de rubídio em direções opostas.

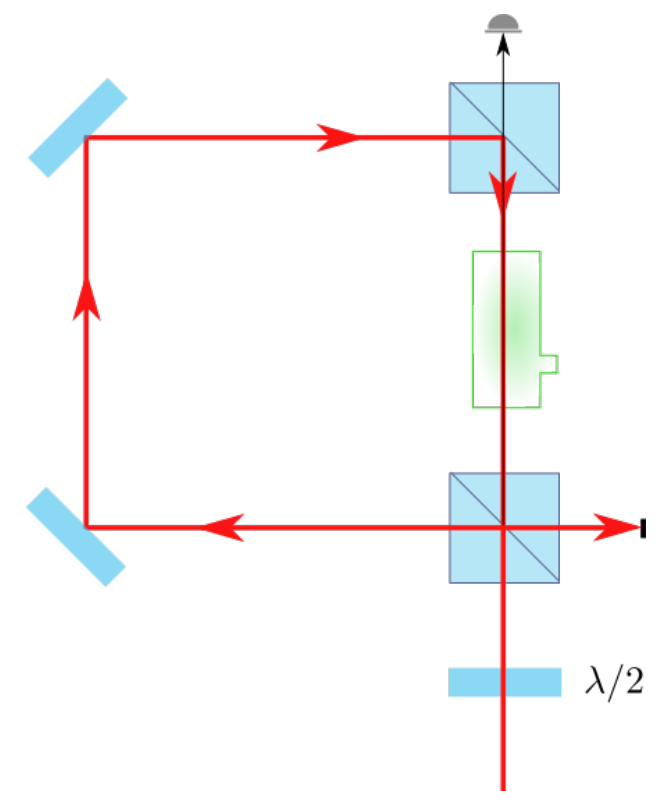

Figura C.1: Ciclo percorrido pelos feixes intenso (em vermelho) e fraco (em preto) da absorção saturada. O feixe fraco possui polarização horizontal e atravessa a célula de rubídio sem sofrer desvios e é detectado ao final do percurso. O feixe intenso possui polarização vertical e percorre o ciclo sendo refletido pelos PBSs e espelhos até atravessar a célula de rubídio e ser refletido para fora do ciclo atingindo um anteparo. 
Dentro da célula de vapor atômico, a uma temperatura T, os átomos estão a princípio no estado fundamental e possuem a distribuição de velocidades de Maxwell-Boltzmann. No entanto, quando os feixes de luz que cruzam a célula de rubídio possuem uma das frequências que acoplam uma transição atômica pode ocorrer interação com os átomos que serão levados ao nível excitado correspondente.

Devido ao efeito Doppler os átomos com velocidade não nula enxergam o feixes intenso e fraco com frequências diferentes. Assim, átomos com velocidades não nulas podem ser excitados por luz em frequências ao redor da frequência de ressonância. Ao se observar o espectro de transmissão de um feixe simples que atravessa a célula de vapor atômico, percebem-se vales ao redor das frequências características da transição dos átomos. Esses vales prejudicam a distinção entre os picos hiperfinos compreendidos em um mesmo vale.

No entanto, átomos parados enxergam os dois feixes com a mesma frequência. A ideia da absorção saturada é utilizar essa característica para criar uma distinção entre os picos hiperfinos no espectro de transmissão.

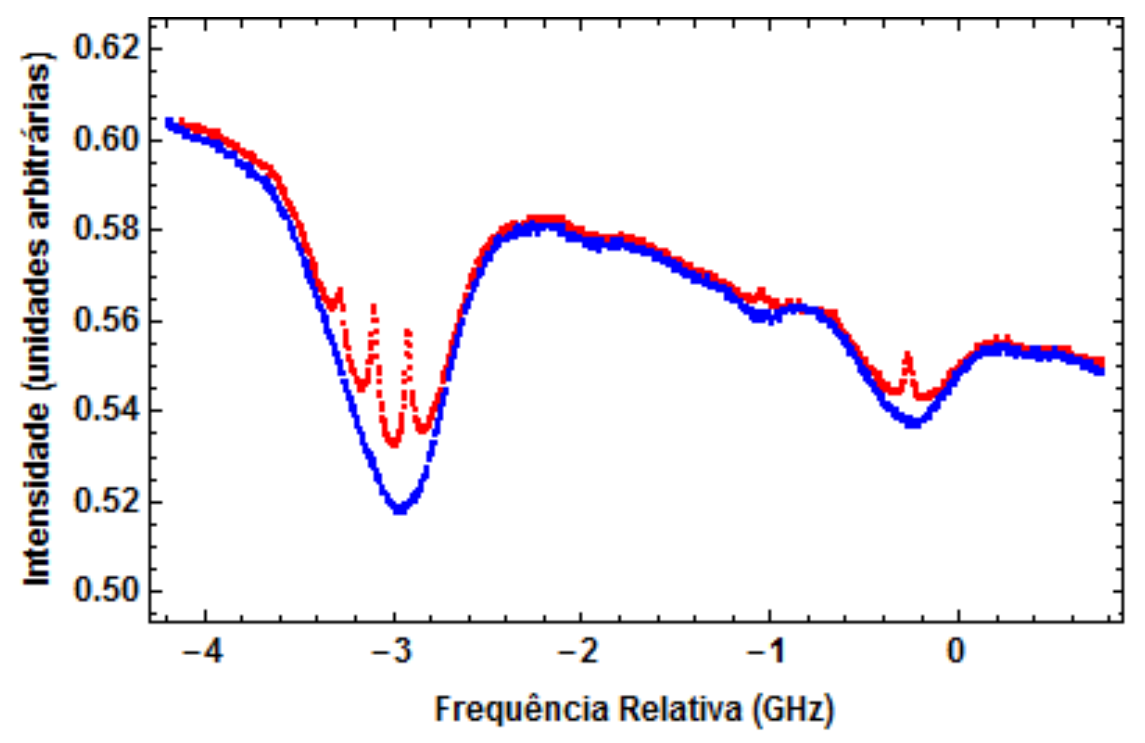

Figura C.2: Espectro de absorção saturada: Podemos ver os picos hiperfinos e crossovers (em vermelho) entre os vales produzidos pelo efeito Doppler (em azul).

O feixe de luz intenso possui maior probabilidade de interagir com os átomos do que o feixe fraco. Assim, quando o feixe de luz intenso atravessa a célula de rubídio em sentido oposto ao feixe fraco ele satura os átomos que estão parados. Por outro lado, os átomos em movimento não são vistos com a mesma frequência pelos dois feixes de luz e, a princípio, não se tornam saturados devido à presença do feixe intenso.

Quando um pico hiperfino reduz a transmissão referente a outro pico hiperfino ocorre um Crossovers. Esses picos se posicionam a uma distância simétrica entre os dois picos hiperfinos responsáveis por formá-lo. 
Quando medimos a transmissão do feixe de menor intensidade após sair do circuito da absorção saturada obtemos picos hiperfinos e Crossovers no interior dos vales da absorção saturada. A Figura C.2 mostra o espectro do feixe fraco para a linha D1 do rubído. Quando bloqueamos o feixe intenso obtemos apenas vales Dopler (em azul). Quando desbloqueamos, os átomos são saturados por esse feixe e vemos os picos hiperfinos e crossovers (em vermelho) entre os vales produzidos pelo efeito Doppler (em azul). 


\section{Apêndice D}

\section{Acoplamento do Ti:safira a fibra ótica}

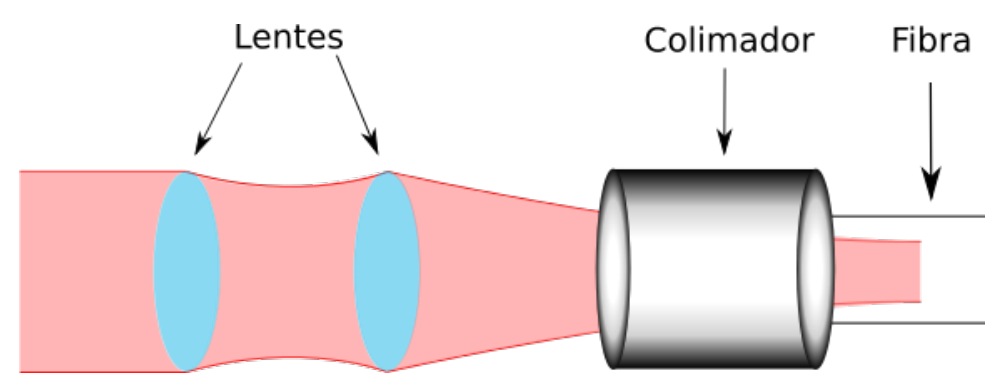

Figura D.1: Acoplamento da fibra ótica.

Nosso feixe de bombeio será fornecido pelo laser Ti:safira que foi levado para o experimento por meio de um acoplamento a uma fibra ótica. Como resultado temos um acoplamento com $60 \%$ de eficiência e $150 \mathrm{~mW}$ de luz na saída da fibra ótica. Nessa seção descrevemos o processo de acoplamento.

Acoplar a fibra ótica consiste em fazer um acordo de modo para que o feixe que sai do Ti:safira esteja colimado e possua o diâmetro ideal para entrar no colimador. O colimador é um componente ótico que consiste de um conjunto de lentes que farão outro acordo de modo para que o feixe entre colimado e com diâmetro adequados na fibra ótica. A Figura D.1 mostra esses dois acordos de modo.

Para escolher as lentes utilizadas no primeiro acordo de modo e a posição das mesmas é possível utilizar a lei das matrizes ABCD em cada etapa do caminho ótico do feixe:

$$
q_{f}=\frac{A q_{i}+B}{C q_{i}+D}
$$

Onde $q_{i}$ e $q_{f}$ são os raios de curvatura complexo do feixe de luz dados por:

$$
\frac{1}{q}=\frac{1}{R(z)}-i \frac{\lambda}{\pi W_{0}^{2}}
$$


$\lambda$ representa o comprimento de onda do feixe, $R(z)$ representa o raio de curvatura, $W_{0}$ a cintura do feixe e A,B,C,D são os parâmetros das matrizes de propagação que descrevem o feixe ao longo de seu caminho ótico até chegar ao colimador. Com o auxílio de um programa de computador, calcula-se o tamanho da cintura que o feixe terá na posição em que o colimador se encontra e variando-se a posição e distância focal das lentes ajustamos essa cintura ao valor adequado para o colimador. Infere-se a cintura que o feixe deve ter ao passar pelo colimador acoplando-se luz à fibra e fazendo com que ela saia pelo colimador ao envés de entrar por ele. 


\section{Referências Bibliográficas}

[1] Squeezed light and entangled images from four-wave-mixing in hot rubidium vapor, vol. 7092, 2008.

[2] R. C. Pooser, A. M. Marino, V. Boyer, K. M. Jones, and P. D. Lett, "Low-noise amplification of a continuous-variable quantum state," Phys. Rev. Lett., vol. 103, p. 010501, Jun 2009.

[3] V. Boyer, A. M. Marino, R. C. Pooser, and P. D. Lett, "Entangled images from four-wave mixing," Science, vol. 321, no. 5888, pp. 544-547, 2008.

[4] R. Slusher, L. Hollberg, B. Yurke, J. Mertz, and J. Valley, "Squeezed states in optical cavities: A spontaneous-emission-noise limit," Physical Review A, vol. 31, no. 5, p. 3512, 1985.

[5] A. Aspect, P. Grangier, and G. Roger, "Experimental realization of einstein-podolskyrosen-bohm gedankenexperiment: a new violation of bell's inequalities," Physical review letters, vol. 49, no. 2, p. 91, 1982.

[6] A. K. Ekert, "Quantum cryptography based on bell's theorem," Physical review letters, vol. 67, no. 6, p. 661, 1991.

[7] C. Silberhorn, N. Korolkova, and G. Leuchs, "Quantum key distribution with bright entangled beams," Physical review letters, vol. 88, no. 16, p. 167902, 2002.

[8] C. H. Bennett, G. Brassard, C. Crépeau, R. Jozsa, A. Peres, and W. K. Wootters, "Teleporting an unknown quantum state via dual classical and einstein-podolsky-rosen channels," Physical review letters, vol. 70, no. 13, p. 1895, 1993.

[9] A. Furusawa, J. L. Sørensen, S. L. Braunstein, C. A. Fuchs, H. J. Kimble, and E. S. Polzik, "Unconditional quantum teleportation," Science, vol. 282, no. 5389, pp. 706-709, 1998.

[10] C. W. A. F. S. L. B. S. Pirandola, J. Eisert, "Advances in quantum teleportation," Nature Photonics, vol. 9, p. 641-652, Sept 2015. 
[11] A. I. Lvovsky, B. Sanders, and W. Tittel, "Optical quantum memory," Nat. Phot., vol. 3, pp. 706-714, 2009.

[12] S. Reynaud, C. Fabre, and E. Giacobino, "Quantum fluctuations in a two-mode parametric oscillator," JOSA B, vol. 4, no. 10, pp. 1520-1524, 1987.

[13] J. Laurat, L. Longchambon, C. Fabre, and T. Coudreau, "Experimental investigation of amplitude and phase quantum correlations in a type ii optical parametric oscillator above threshold: from nondegenerate to degenerate operation," Optics letters, vol. 30, no. 10, pp. 1177-1179, 2005.

[14] J. Laurat, T. Coudreau, G. Keller, N. Treps, and C. Fabre, "Effects of mode coupling on the generation of quadrature einstein-podolsky-rosen entanglement in a type-ii optical parametric oscillator below threshold," Physical Review A, vol. 71, no. 2, p. 022313, 2005.

[15] M. Martinelli, Compressão de Ruído Quântico e Efeitos Transversos em Osciladores Paramétricos Óticos. PhD thesis, Universidade de São Paulo, 2002.

[16] A. S. Villar, L. S. Cruz, K. N. Cassemiro, M. Martinelli, and P. Nussenzveig, "Generation of bright two-color continuous variable entanglement," Phys. Rev. Lett., vol. 95, p. 243603, Dec 2005.

[17] A. S. Villar, M. Martinelli, C. Fabre, and P. Nussenzveig, "Direct production of tripartite pump-signal-idler entanglement in the above-threshold optical parametric oscillator," Phys. Rev. Lett., vol. 97, p. 140504, Oct 2006.

[18] A. S. Coelho, F. A. S. Barbosa, K. N. Cassemiro, A. S. Villar, M. Martinelli, and P. Nussenzveig, "Three-color entanglement," Science, vol. 326, no. 5954, pp. 823-826, 2009.

[19] A. S. O. Coelho, "Emaranhamento tripartite no oscilador paramétrico Ótico," Master's thesis, Universidade de Sao Paulo, 2009.

[20] Strong quantum correlations in four wave mixing in 85Rb vapor, vol. 7727, 2010.

[21] R. Pooser, A. Marino, V. Boyer, K. Jones, and P. Lett, "Quantum correlated light beams from non-degenerate four-wave mixing in an atomic vapor: the $\mathrm{d} 1$ and $\mathrm{d} 2$ lines of $85 \mathrm{rb}$ and 87 rb," Optics express, vol. 17, no. 19, pp. 16722-16730, 2009.

[22] K. Jensen, W. Wasilewski, H. Krauter, T. Fernholz, B. M. Nielsen, M. Owari, M. B. Plenio, A. Serafini, M. Wolf, and E. Polzik, "Quantum memory for entangled continuous-variable states," Nature Physics, vol. 7, no. 1, p. 13, 2011. 
[23] A. Kitagawa, M. Takeoka, M. Sasaki, and A. Chefles, "Entanglement evaluation of nongaussian states generated by photon subtraction from squeezed states," Physical Review $A$, vol. 73 , no. 4, p. 042310, 2006.

[24] F. Dell'Anno, S. De Siena, L. Albano, and F. Illuminati, "Continuous-variable quantum teleportation with non-gaussian resources," Physical Review A, vol. 76, no. 2, p. 022301, 2007.

[25] M. O. Scully and M. S. Zubairy, Quantum Optics. Cambridge: Cambridge University Press, 0091997.

[26] G. M. D.F.Walls, Quantum Optics. Springer-Verlag, 1994.

[27] A. de Sousa Villar, Emaranhamento Multicolor entre Feixes Intensos de Luz. PhD thesis, USP, 2007.

[28] A. S. O. Coelho, Emaranhamento multicor para redes de informação quântica. PhD thesis, USP, 2013.

[29] C. C. Gerry and P. L. Knight, Introduction to quantum optics. Cambridge University Press, 2004.

[30] M. Nussenzveig, Curso de Fúsica Básica 4 - Ótica Relatividade Fúsica Quântica. Edgar Blücher, 1998.

[31] H. Bachor and T. Ralph, A guide to Experiments in Quantum Optics. Wiley, 2004.

[32] C. W. J. Beenakker and M. Büttiker, "Suppression of shot noise in metallic diffusive conductors," Phys. Rev. B, vol. 46, pp. 1889-1892, Jul 1992.

[33] U. Leonhardt, Essential Quantum Optics: From Quantum Measurements to Black Holes. Cambridge University Press, 2010.

[34] F. A. S. Barbosa, A. S. Coelho, K. N. Cassemiro, P. Nussenzveig, C. Fabre, A. S. Villar, and M. Martinelli, "Quantum state reconstruction of spectral field modes: Homodyne and resonator detection schemes," Phys. Rev. A, vol. 88, p. 052113, November 2013.

[35] R. Simon, "Peres-Horodecki separability criterion for continuous variable systems," Physical Review Letters, vol. 84, pp. 2726-2729, Mar. 2000. arXiv: quant-ph/9909044.

[36] L. Madsen and K. Mølmer, "Continuous measurements on continuous variable quantum systems: The gaussian description," arXiv preprint quant-ph/0511154, 2005.

[37] A. I. Lvovsky and M. G. Raymer, "Continuous-variable optical quantum-state tomography," Reviews of Modern Physics, vol. 81, no. 1, p. 299, 2009. 
[38] R. J. Glauber, "The quantum theory of optical coherence," Phys. Rev., vol. 130, pp. 25292539, Jun 1963.

[39] L. Mandel and E. Wolf, Optical Coherence and Quantum Optics. Cambridge University Press, 1995.

[40] Q. Glorieux, ETUDE THEORIQUE ET EXPERIMENTALE DES CORRELATIONS QUANTIQUES OBTENUES PAR MELANGE A QUATRE ONDES DANS UNE VAPEUR ATOMIQUE. PhD thesis, Université Paris-Diderot-Paris VII, 2010.

[41] G. Hernández, Fabry-perot interferometers. No. 3, Cambridge University Press, 1988.

[42] T. Frank, Springer Handbook of Lasers and Optics. Springer, 2012.

[43] A. Einstein, B. Podolsky, and N. Rosen, "Can quantum-mechanical description of physical reality be considered complete?," Phys. Rev., vol. 47, p. 777, 1935.

[44] N. Bohr, "Can quantum mechanical description of physical reality be considered complete?," Phys. Rev., vol. 48, p. 696, 1935.

[45] J. S. Bell, "On the einstei-podolsky-rosen paradox," Physics, vol. 1, p. 195, 1964.

[46] L.-M. Duan, G. Giedke, J. I. Cirac, and P. Zoller, "Inseparability criterion for continuous variable systems," Physical Review Letters, vol. 84, no. 12, p. 2722, 2000.

[47] A. Peres, "Separability criterion for density matrices," Phys. Rev. Lett., vol. 77, pp. 14131415, Aug 1996.

[48] R.Simon, "Peres-horodecki separability criterion for continuous variable systems," Phys. Rev. Lett., no. 84, p. 2726, 2000.

[49] G. Adesso, Entanglement of Gaussian State. PhD thesis, Università degli Studi diSalerno, 2006.

[50] R. W. Boyd, Nonlinear Optics. Academic Press, third ed., 2008.

[51] C. F. McCormick, A. M. Marino, V. Boyer, and P. D. Lett, "Strong low-frequency quantum correlations from a four-wave-mixing amplifier," Phys. Rev. A, vol. 78, p. 043816, Oct 2008.

[52] T. Debuisschert, A. Sizmann, E. Giacobino, and C. Fabre, "Type-ii continuous-wave optical parametric oscillators: oscillation and frequency-tuning characteristics," J. Opt. Soc. Am. $B$, vol. 10, p. 1668, 1993.

[53] L. F. Muñoz-Martínez, F. A. S. Barbosa, A. S. Coelho, L. Ortiz-Gutiérrez, M. Martinelli, P. Nussenzveig, and A. S. Villar, "Exploring six modes of an optical parametric oscillator," Physical Review A, vol. 98, no. 2, p. 023823, 2018. 
[54] M. G. Paris, F. Illuminati, A. Serafini, and S. De Siena, "Purity of gaussian states: Measurement schemes and time evolution in noisy channels," Physical Review A, vol. 68, no. 1, p. 012314, 2003.

[55] T. Golubeva, Y. Golubev, C. Fabre, and N. Treps, "Quantum state of an injected tropo above threshold: purity, glauber function and photon number distribution," The European Physical Journal D, vol. 46, no. 1, pp. 179-193, 2008.

[56] H. A. R. Páez, "Caracterização da amplificação paramétrica no processo de mistura de quatro ondas na configuração duplo-lambda em vapor de rubídio," Master's thesis, Universidade de São Paulo, 2017.

[57] A. M. Guerrero, "Oscilador paramétrico ótico baseado em mistura de quatro ondas em vapor de rubídio," Master's thesis, Universidade de São Paulo, 2017.

[58] F. C. D. de Moraes, "Construção e caracterização de um laser contínuo de titânio-safira," Master's thesis, Universidade de São Paulo, 2013.

[59] R. B. de Andrade, "Construção de um oscilador paramétrico Ótico para uma interface Átomo luz," Master's thesis, Universidade de São Paulo, 2013.

[60] C. J. Foot, Atomic physics, vol. 7. Oxford University Press, 2005. 